\title{
Application of Allylzinc Reagents as Nucleophiles in Matteson Homologations
}

\author{
Oliver Andler ${ }^{a}$ and Uli Kazmaier ${ }^{a, b} *$ \\ ${ }^{a}$ Saarland University, Organic Chemistry I, Campus, Building C4.2, D-66123 Saarbrücken, Germany \\ ${ }^{b}$ Helmholtz Institute for Pharmaceutical Research Saarland (HIPS), Saarland University Campus, Building \\ C8.1, 66123 Saarbrücken, Germany \\ Email: u.kazmaier@mx.uni-saarland.de
}

Experimental Section $\quad$ S2

$\begin{array}{ll}\text { General remarks } & \text { S2 }\end{array}$

$\begin{array}{ll}\text { General procedures } & \text { S2 }\end{array}$

Matteson homologation using allylmagnesium bromide $\quad$ S5

Matteson homologation using substituted allylzinc reagents $\quad$ S6

Matteson homologation and modification of $\alpha$-allylated boronic esters $\quad$ S33

Copies of the NMR spectra, HPLC and GC chromatograms $\quad$ S38 


\section{Experimental Section}

General remarks: All air- or moisture-sensitive reactions were carried out in oven-dried glassware $\left(75^{\circ} \mathrm{C}\right)$ under an atmosphere of nitrogen. Dried solvents were distilled before use: THF was distilled from sodium/benzophenone, diisopropylamine was dried with $\mathrm{CaH}_{2}$ before distillation. Anhydrous dichloromethane (DCM) and DMSO were purchased from Acros Organics and stored under nitrogen. Petroleum ether $\left(40-60^{\circ} \mathrm{C}\right)$, pentane and ethyl acetate were distilled prior to use. Lithium chloride was fused in vacuo $(0.1 \mathrm{mbar})$ prior to use. The products were purified by flash chromatography on silica gel (Macherey-Nagel 60, 0.063-0.2 mm or 0.04-0.063 mm) or, in case of $\beta$-hydroxy ketone 30a, $\mathrm{Al}_{2} \mathrm{O}_{3}$ (Merck 90, active basic, activity stage 1, 0.063-0.2 mm). For reversed-phase flash chromatography, a Büchi Reveleris ${ }^{\circledR}$ Prep Chromatography System and Büchi FlashPure Select C18 (30 $\mu \mathrm{m}$ spherical) columns were used. Analytical TLC was performed on pre-coated silica gel or, in case of $\beta$-hydroxy ketone 30a, $\mathrm{Al}_{2} \mathrm{O}_{3}$ plates (Macherey-Nagel, Polygram ${ }^{\circledR} \mathrm{SIL} \mathrm{G} / \mathrm{UV}_{254}\left[\mathrm{SiO}_{2}\right]$ or Polygram ${ }^{\circledR}$ ALOX $\left.\mathrm{N} / \mathrm{UV}_{254}\left[\mathrm{Al}_{2} \mathrm{O}_{3}\right]\right)$. Visualization was accomplished with UV-light, $\mathrm{KMnO}_{4}$ solution or cerium(IV)/ammonium molybdate solution. Melting points were determined with a MELTEMP II apparatus and are uncorrected. ${ }^{1} \mathrm{H},{ }^{13} \mathrm{C}$ and ${ }^{11} \mathrm{~B}$ NMR spectra were recorded with Bruker AV 400 Ultra Shield $\left[400 \mathrm{MHz}\left({ }^{1} \mathrm{H}\right), 100 \mathrm{MHz}\left({ }^{13} \mathrm{C}\right)\right.$ and $\left.128 \mathrm{MHz}\left({ }^{11} \mathrm{~B}\right)\right]$, Bruker AV $500\left[500 \mathrm{MHz}\left({ }^{1} \mathrm{H}\right)\right.$ and $\left.125 \mathrm{MHz}\left({ }^{13} \mathrm{C}\right)\right]$ or Bruker Avance Neo $500\left[500 \mathrm{MHz}\left({ }^{1} \mathrm{H}\right)\right.$ and $\left.125 \mathrm{MHz}\left({ }^{13} \mathrm{C}\right)\right]$ spectrometers in $\mathrm{CDCl}_{3}$ or $\mathrm{CD}_{3} \mathrm{CN}$. Chemical shifts are reported in ppm $(\delta)$ with respect to TMS, and $\mathrm{CHCl}_{3}$ or acetonitrile- $\mathrm{d}_{2}$ was used as the internal standard. Selected signals for the minor regioisomers or double bond isomers are extracted from the spectra of the isomeric mixture. Peaks were assigned using ${ }^{1} \mathrm{H},{ }^{1} \mathrm{H} \mathrm{COSY}$, multiplicity edited ${ }^{1} \mathrm{H},{ }^{13} \mathrm{C} \mathrm{HSQC}$, ${ }^{1} \mathrm{H},{ }^{13} \mathrm{C}$ HMBC and selective $1 \mathrm{D}$ CSSF TOCSY spectra. Enantiomeric ratios were determined by HPLC on a Merck-Hitachi D-7000 chromatograph [column: Daicel Chiralcel OD-H (250 x $4.6 \mathrm{~mm}, 5 \mu \mathrm{m})]$. Diastereomeric and regioisomeric ratios were determined by ${ }^{1} \mathrm{H} \mathrm{NMR}$ or GC-FID on a Shimadzu GC-2010 chromatograph [column: Varian CP-Chirasil-Dex CB (25 m x $0.25 \mathrm{~mm}, 0.25 \mu \mathrm{m}$ ID); carrier gas $\mathrm{N}_{2}$ ]. Optical rotations were measured with a PerkinElmer 341 or Jasco P-2000 polarimeter at the sodium D line $(589 \mathrm{~nm}) . \alpha_{D}^{20}$ values are given in $10^{-1} \mathrm{deg} \mathrm{cm}^{2} \mathrm{~g}^{-1}$. Mass spectra were recorded with a Finnigan MAT 95 sector field spectrometer $(\mathrm{CI})$.

\section{General procedure for Matteson homologations (GP 1).}

LDA solution: $n$-butyllithium (1.6 M in hexanes, 1.25 eq.) was added dropwise to a solution of diisopropylamine (1.35 eq.) in anhydrous THF $(0.2 \mathrm{ml} / \mathrm{mmol})$ at $-40{ }^{\circ} \mathrm{C}$. The mixture was allowed to warm to room temperature and stirred for $20 \mathrm{~min}$.

$\underline{\mathrm{ZnCl}} 2$ solution: In a Schlenk flask, $\mathrm{ZnCl}_{2}$ was heated to approximately $250{ }^{\circ} \mathrm{C}$ in vacuo (0.1 mbar) with stirring over 5-10 min using a heat gun. After cooling to room temperature with stirring, the flask was refilled with nitrogen and the drying procedure was repeated for a second time. The dried $\mathrm{ZnCl}_{2}$ was dissolved in anhydrous THF $\left(0.6 \mathrm{ml} / \mathrm{mmol} \mathrm{ZnCl}_{2}\right)$.

Homologation: The freshly prepared LDA solution was slowly added to a solution of the boronic ester (1.0 eq.) and anhydrous dichloromethane (3.0 eq.) in anhydrous THF $(1.4 \mathrm{ml} / \mathrm{mmol})$ at $-40{ }^{\circ} \mathrm{C}$. After stirring at this temperature for $10 \mathrm{~min}$, the zinc chloride solution was added and the mixture was stirred for $2 \mathrm{~h}$ at room temperature.

GP1a: Isolation of the crude $\alpha$-chloroboronic ester: Saturated $\mathrm{NH}_{4} \mathrm{Cl}$ was added to the mixture of the homologation step. After stirring for $5 \mathrm{~min}$, the layers were separated and the aqueous layer was extracted twice with pentane. The combined organic layers were dried over $\mathrm{Na}_{2} \mathrm{SO}_{4}$ and concentrated in vacuo. The residue was directly used in the next step without further purification. 
GP1b: Reaction with Grignard reagents: The mixture was cooled to $-78{ }^{\circ} \mathrm{C}$ (if AllylMgBr was used) or $0{ }^{\circ} \mathrm{C}$ (if EtMgBr was used) and the Grignard reagent (2.5 eq.) was slowly added. After stirring for $1 \mathrm{~h}$ at $-78{ }^{\circ} \mathrm{C}$ (if AllylMgBr was used) or $2 \mathrm{~d}$ at room temperature (if EtMgBr was used), saturated $\mathrm{NH}_{4} \mathrm{Cl}$ was added. The biphasic mixture was stirred for $5 \mathrm{~min}$, then the layers were separated and the aqueous layer was extracted twice with pentane. The combined organic layers were dried over $\mathrm{Na}_{2} \mathrm{SO}_{4}$ and concentrated in vacuo. If necessary, the crude product was purified by flash chromatography. The products were sufficiently pure to be used in the next step. A sample of the boronic ester was further purified by reversed-phase flash chromatography for analytical purposes if required.

\section{General procedure for the oxidation of boronic esters to alcohols (GP 2).}

To a solution of the boronic ester (1.0 eq.) in THF $(2 \mathrm{ml} / \mathrm{mmol})$ were added hydrogen peroxide (33\% in water, 5.0 eq.) and a solution of sodium hydroxide $(5.0$ eq.) in water $(2 \mathrm{ml} / \mathrm{mmol})$. The mixture was allowed to warm to room temperature and stirred until TLC control indicated full conversion. Brine was added and the aqueous layer was extracted three times with diethyl ether. The combined organic layers were dried over $\mathrm{Na}_{2} \mathrm{SO}_{4}$, concentrated in vacuo and purified by flash chromatography.

If the desired alcohol and the chiral auxiliary $(S, S)$-DICHED were not separable via chromatography, $(S, S)$-DICHED was converted to its methylboronic ester as follows: The crude product was dissolved in diethyl ether $(5 \mathrm{ml} / \mathrm{mmol})$ and methylboronic acid (1.2 eq.) and an excess of $\mathrm{MgSO}_{4}$ were added at room temperature. After stirring at room temperature overnight, the mixture was filtered, concentrated in vacuo and purified by flash chromatography.

\section{General procedure for the preparation of allylzinc reagents by zinc insertion (GP 3).}

A modified procedure by Knochel et al. was used. ${ }^{1}$

Lithium chloride (1.1 eq.) and zinc dust (2.0 eq.) were suspended in anhydrous THF $(1 \mathrm{ml} / \mathrm{mmol})$. To activate the zinc dust, 1,2-dibromomethane ( 2 mol- $\%)$ was added and the mixture was heated using a heat gun until it started to gently boil. After cooling to room temperature, TMSCl (5 mol-\%) was added and the suspension was again heated until it started to gently boil. The mixture was allowed to cool to room temperature and a solution of the substituted allyl bromide (1.0 eq.) in anhydrous THF $(1 \mathrm{ml} / \mathrm{mmol})$ was added dropwise, resulting in an exothermic reaction, and stirred for $2-3 \mathrm{~h}$ at room temperature. Excess zinc dust was allowed to settle and the supernatant solution was directly used in the next step. The concentration of the organozinc solution was determined by iodometric titration. ${ }^{2}$

\section{General procedure for the substitution of $\alpha$-chloroboronic esters with allylzinc nucleophiles (GP 4).}

The allylzinc solution (2.0 eq.) was added dropwise to a solution of the crude $\alpha$-chloroboronic ester (1.0 eq.) in anhydrous THF $(3 \mathrm{ml} / \mathrm{mmol})$ at room temperature. After stirring at the same temperature for $14-18 \mathrm{~h}$, saturated $\mathrm{NH}_{4} \mathrm{Cl}$ was added. The biphasic mixture was stirred for $5 \mathrm{~min}$, then the layers were separated and the aqueous layer was extracted twice with pentane. The combined organic layers were dried over $\mathrm{Na}_{2} \mathrm{SO}_{4}$ and concentrated in vacuo. The crude product was purified by flash chromatography. The products were sufficiently pure to be used in the next step. If necessary, a sample of the aldehyde or ketone was further purified by reversed-phase flash chromatography for analytical purposes.

\footnotetext{
${ }^{1}$ Sämann, C.; Knochel, P., A Convenient Synthesis of alpha-Substituted beta,gamma-Unsaturated Ketones and Esters via the Direct Addition of Substituted Allylic Zinc Reagents Prepared by Direct Insertion. Synthesis 2013, 45, 1870-1876.

${ }^{2}$ Krasovskiy, A.; Knochel, P., Convenient titration method for organometallic zinc, magnesium, and lanthanide reagents. Synthesis 2006, 890-891.
} 
General procedure for the ozonolysis of $\alpha$-allylated boronic esters (GP 5).

Ozone was bubbled through a solution of the $\alpha$-allylated boronic ester (1.0 eq.) in DCM $(20 \mathrm{ml} / \mathrm{mmol})$ at $-78{ }^{\circ} \mathrm{C}$ until the solution was colored blue (1.5-3 min). Excess ozone was removed by bubbling through with oxygen and triphenylphosphine (1.2 eq.) was added. The mixture was warmed to room temperature over 15-25 min and the solvent was removed in vacuo. After purification of the residue by normal-phase flash chromatography, the product was sufficiently pure to be used in the next step. For analytical purposes, a sample of the aldehyde or ketone was further purified by reversed-phase flash chromatography to remove trace impurities of excess triphenylphosphine. 


\section{Matteson homologation using allylmagnesium bromide}

\section{(4S,5S)-4,5-Dicyclohexyl-2-[(S)-1-phenylhex-5-en-3-yl]-1,3,2-dioxaborolane (2)}

$(4 S, 5 S)$-4,5-dicyclohexyl-2-phenethyl-1,3,2-dioxaborolane 1 was prepared as described by Molander et $_{\text {al. }}{ }^{3}$

According to GP $1 \mathrm{~b} 300 \mathrm{mg}(882 \mu \mathrm{mol})$ boronic ester $1,170 \mu \mathrm{l}(\rho=1.32 \mathrm{~g} / \mathrm{ml}, 2.64 \mathrm{mmol})$ anhydrous DCM, $689 \mu \mathrm{l}(1.6 \mathrm{M}$ in hexanes, $1.10 \mathrm{mmol}) n$-butyllithium, $170 \mu \mathrm{l}(\rho=0.71 \mathrm{~g} / \mathrm{ml}$, $1.19 \mathrm{mmol})$ diisopropylamine and $240 \mathrm{mg}(1.76 \mathrm{mmol})$ zinc chloride were reacted. To the $\alpha$-chloroboronic ester solution were added $2.20 \mathrm{ml}(2.20 \mathrm{mmol}, 2.5 \mathrm{eq}$.) allylmagneisum bromide (1.0 M in diethyl ether) at $-78{ }^{\circ} \mathrm{C}$. After stirring at the same temperature for $1 \mathrm{~h}$, the reaction was worked up to obtain crude 2 in $99 \%$ yield $(344 \mathrm{mg}, 872 \mu \mathrm{mol}$ ) as a colorless oil, $\alpha_{D}^{20}=-45.3\left(\mathrm{c}=1.0, \mathrm{CHCl}_{3}\right) .2$ was used in the next step without further purification.

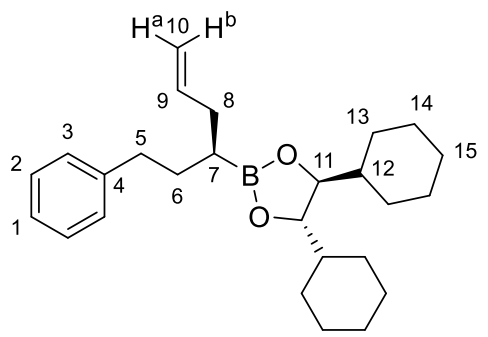

${ }^{1} \mathbf{H}$ NMR $\left(400 \mathrm{MHz}, \mathrm{CDCl}_{3}\right): \delta=0.98(\mathrm{~m}, 2 \mathrm{H}, 14-\mathrm{H}), 1.07(\mathrm{~m}, 2 \mathrm{H}, 13-\mathrm{H}), 1.12-1.40(\mathrm{~m}, 9 \mathrm{H}$, 7-H, 12-H, 13-H', 14-H'), 1.55-1.72 (m, $\left.5 \mathrm{H}, 6-\mathrm{H}_{\mathrm{a}}, 13-\mathrm{H}^{\prime}, 15-\mathrm{H}\right), 1.72-1.88$ (m, $7 \mathrm{H}, 6-\mathrm{H}_{\mathrm{b}}$, 13-H' ', 14-H', 15-H'), 2.22 (m, 2 H, 8-H), 2.63 (m, 2 H, 5-H), 3.85 (m, 2 H, 12-H), 4.95 (ddt, $\left.{ }^{3} J_{10 \mathrm{a}, 9}=10.1 \mathrm{~Hz},{ }^{2} J_{10 \mathrm{a}, 8}=2.2 \mathrm{~Hz},{ }^{4} J_{10 \mathrm{a}, 8}=1.1 \mathrm{~Hz}, 1 \mathrm{H}, 10-\mathrm{H}_{\mathrm{a}}\right), 5.02\left(\mathrm{ddt},{ }^{3} J_{10 \mathrm{~b}, 9}=17.0 \mathrm{~Hz}\right.$, $\left.{ }^{2} J_{10 \mathrm{~b}, 10 \mathrm{a}} \approx{ }^{4} J_{10 \mathrm{~b}, 8}=1.8 \mathrm{~Hz}, 1 \mathrm{H}, 10-\mathrm{H}_{\mathrm{b}}\right), 5.81\left(\mathrm{ddt},{ }^{3} J_{9,10 \mathrm{~b}}=17.1 \mathrm{~Hz},{ }^{3} J_{9,10 \mathrm{a}}=10.1 \mathrm{~Hz},{ }^{3} J_{9,8}=\right.$ $6.9 \mathrm{~Hz}, 1 \mathrm{H}, 9-\mathrm{H}), 7.13-7.21$ (m, $3 \mathrm{H}, 1-\mathrm{H}, 3-\mathrm{H}), 7.27$ (m, $2 \mathrm{H}, 2-\mathrm{H})$.

${ }^{13} \mathrm{C} \mathrm{NMR}\left(100 \mathrm{MHz}, \mathrm{CDCl}_{3}\right): \delta=25.9$ (t, C-14), 26.0 (t, C-13), 26.5 (t, C-15), 27.6 (t, C-14'), 28.5 (t, C-13'), 33.0 (t, C-6), 35.5 (t, C-8), 35.5 (t, C-5), 43.1 (d, C-12), 83.4 (d, C-11), 115.0 (t, C-10), 125.5 (d, C-1), 128.2 (d, C-2), 128.4 (d, C-3), 138.5 (d, C-9), 143.1 (s, C-4).

The signal of C-7 could not be detected.

HRMS (CI) $\mathrm{m} / \mathrm{z}$ calcd for $\mathrm{C}_{26} \mathrm{H}_{39} \mathrm{O}_{2} \mathrm{~B}[\mathrm{M}]^{+}:$394.3038, found: 394.3045 .

\section{(S)-1-Phenylhex-5-en-3-ol (3)}

$321 \mathrm{mg}(814 \mu \mathrm{mol})$ crude boronic ester $2,378 \mu \mathrm{l}(33 \%$ in water, $\rho=1.11 \mathrm{~g} / \mathrm{ml}, 4.07 \mathrm{mmol})$ hydrogen peroxide and $163 \mathrm{mg}$ ( $4.07 \mathrm{mmol})$ sodium hydroxide were reacted according to GP 2 . After $2 \mathrm{~h}$, the reaction was worked up and the crude product was purified by flash chromatography (petroleum ether, ethyl acetate $85: 15)$ to give 3 (98 \% ee) in $81 \%$ yield $(116 \mathrm{mg}, 658 \mu \mathrm{mol})$ as a colorless oil, $\mathrm{R}_{\mathrm{f}}=0.28$ (petroleum ether, ethyl acetate $\left.8: 2\right) ; \alpha_{D}^{20}=$ $-25.1\left(\mathrm{c}=0.53, \mathrm{CHCl}_{3}\right)$.

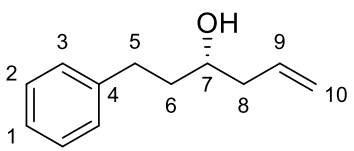

${ }^{1} \mathbf{H}$ NMR (400 MHz, $\left.\mathrm{CDCl}_{3}\right): \delta=1.67$ (bs, $\left.1 \mathrm{H}, \mathrm{OH}\right), 1.79$ (m, $\left.2 \mathrm{H}, 6-\mathrm{H}\right), 2.19\left(\mathrm{~m}, 1 \mathrm{H}, 8-\mathrm{H}_{\mathrm{a}}\right)$, $2.33\left(\mathrm{~m}, 1 \mathrm{H}, 8-\mathrm{H}_{\mathrm{b}}\right), 2.70\left(\mathrm{~m}, 1 \mathrm{H}, 5-\mathrm{H}_{\mathrm{a}}\right), 2.82\left(\mathrm{~m}, 1 \mathrm{H}, 5-\mathrm{H}_{\mathrm{b}}\right), 3.68(\mathrm{~m}, 1 \mathrm{H}, 7-\mathrm{H}), 5.11-5.20$ (m, 2 H, 10-H), $5.82(\mathrm{~m}, 1 \mathrm{H}, 9-\mathrm{H}), 7.16-7.25$ (m, $3 \mathrm{H}, 1-\mathrm{H}, 3-\mathrm{H}), 7.29$ (m, $1 \mathrm{H}, 2-\mathrm{H})$.

\footnotetext{
${ }^{3}$ Molander, G. A.; Wisniewski, S. R., Stereospecific Cross-Coupling of Secondary Organotrifluoroborates: Potassium 1-(Benzyloxy)alkyltrifluoroborates. J. Am. Chem. Soc. 2012, 134, 16856-16868.
} 
${ }^{13} \mathrm{C} \mathrm{NMR}\left(100 \mathrm{MHz}, \mathrm{CDCl}_{3}\right): \delta=32.0(\mathrm{t}, \mathrm{C}-5), 38.4(\mathrm{t}, \mathrm{C}-6), 42.1$ (t, C-8), $69.9(\mathrm{~d}, \mathrm{C}-7), 118.3$ (t, C-10), 125.8 (d, C-1), 128.4 (d, C-2), 128.4 (d, C-3), 134.6 (d, C-9), 142.0 (s, C-4).

HPLC (Chiralcel OD-H $5 \mu \mathrm{m}$, hexane/ $\mathrm{PrOH} 9: 1,1.0 \mathrm{ml} / \mathrm{min}, 20{ }^{\circ} \mathrm{C}$ ): $\mathrm{t}_{\mathrm{R}}(S)-3=7.63 \mathrm{~min}$, $\mathrm{t}_{\mathrm{R}}(R)-3=11.53$ min.

HRMS (CI) m/z calcd for $\mathrm{C}_{12} \mathrm{H}_{17} \mathrm{O}[\mathrm{M}+\mathrm{H}]^{+}:$177.1274, found: 177.1285 .

A racemic sample of $\mathbf{3}$ was prepared as described by Baran et al. ${ }^{4}$

\section{Matteson homologation using substituted allylzinc reagents}

\section{Crotylzinc bromide lithium chloride (4c)}

$1.35 \mathrm{~g}$ (10.0 mmol) trans-crotyl bromide ${ }^{5}, 466 \mathrm{mg}(11.0 \mathrm{mmol})$ lithium chloride, $1.31 \mathrm{~g}$ ( $20.0 \mathrm{mmol})$ zinc dust, $17 \mu \mathrm{l}(\rho=2.18 \mathrm{~g} / \mathrm{ml}, 200 \mu \mathrm{mol})$ 1,2-dibromoethane and $64 \mu \mathrm{l}(\rho=$ $0.856 \mathrm{~g} / \mathrm{ml}, 500 \mu \mathrm{mol}$ ) TMSCl were reacted according to GP3. After stirring for $2 \mathrm{~h}, \mathbf{4 c}$ was obtained as a $0.43 \mathrm{M}$ solution in THF ( $86 \%$ yield) according to iodometric titration.

\section{(4S,5S)-4,5-Dicyclohexyl-2-[(S,Z)-1-phenylhept-5-en-3-yl]-1,3,2-dioxaborolane (5)}

\section{(4S,5S)-4,5-Dicyclohexyl-2-[(3S)-4-methyl-1-phenylhex-5-en-3-yl]-1,3,2-dioxaborolane} (6)

(4S,5S)-2-[(R)-1-Chloro-3-phenylpropyl]-4,5-dicyclohexyl-1,3,2-dioxaborolane 7 was prepared as decribed by Molander et $\mathrm{ll}^{3}{ }^{3}$

According to GP $480 \mathrm{mg}(206 \mu \mathrm{mol}) \alpha$-chloroboronic ester 7 were reacted with $2.94 \mathrm{ml}$ $(411 \mu \mathrm{mol})$ allylzinc reagent $\mathbf{4 c}(0.14 \mathrm{M}$ in THF). After stirring for $19 \mathrm{~h}$, the reaction was worked up and the crude product was purified by flash chromatography (petroleum ether, ethyl acetate 98:2) to give 5 (regioisomeric ratio 5/6 > 96:4 according to ${ }^{1} \mathrm{H}$ NMR of the boronic ester, $E: Z=19: 81$ according to ${ }^{1} \mathrm{H}$ NMR after oxidation to alcohol 5') in $76 \%$ yield (64 mg, $157 \mu \mathrm{mol}$ ) as a colorless oil, $\mathrm{R}_{\mathrm{f}}=0.17$ (petroleum ether, ethyl acetate 98:2).

major regioisomer (5):

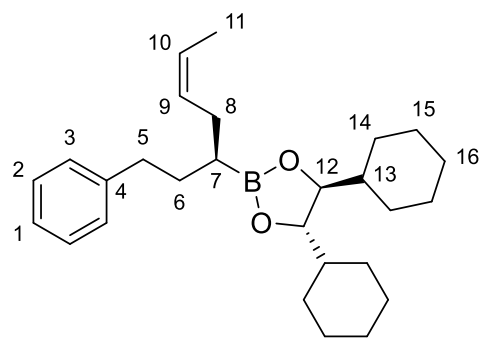

major double bond isomer ( $Z$ isomer):

${ }^{1} \mathbf{H}$ NMR (400 MHz, CDCl 3$): \delta=0.97(\mathrm{~m}, 2 \mathrm{H}, 15-\mathrm{H}), 1.07$ (m, $\left.2 \mathrm{H}, 14-\mathrm{H}\right), 1.12-1.26(\mathrm{~m}, 7 \mathrm{H}$, 7-H, 14-H', 15-H'), $1.32(\mathrm{~m}, 2 \mathrm{H}, 13-\mathrm{H}), 1.57-1.86\left(\mathrm{~m},{ }^{3} J_{11,10}=5.9 \mathrm{~Hz}, 15 \mathrm{H}, 6-\mathrm{H}, 11-\mathrm{H}\right.$, 14-H', 15-H' ' 16-H), 2.18 (m, 2 H, 8-H), 2.62 (m, 2 H, 5-H), 3.85 (m, 2 H, 12-H), 5.34-5.50 (m, 2 H, 9-H, 10-H), 7.12-7.22 (sh, 3 H, 1-H, 3-H), 7.27 (m, 2 H, 2-H).

\footnotetext{
${ }^{4}$ Hu, P.; Peters, B. K.; Malapit, C. A.; Vantourout, J. C.; Wang, P.; Li, J.; Mele, L.; Echeverria, P.-G.; Minteer, S. D.; Baran, P. S., Electroreductive Olefin-Ketone Coupling. J. Am. Chem. Soc. 2020, 142, 20979-20986.

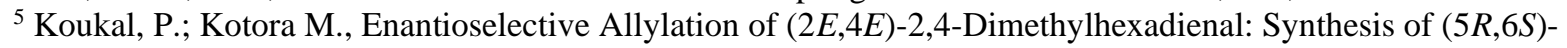
(+)-Pteroenone. Chem. Eur. J. 2015, 21, 7408-7412.
} 
${ }^{13}$ C NMR (100 MHz, $\left.\mathrm{CDCl}_{3}\right): \delta=12.9$ (q, C-11), 25.9 (t, C-15), 26.0 (t, C-14), 26.5 (t, C-16), 27.5 (t, C-15'), 28.4 (t, C-8), 28.5 (t, C-14'), 33.1 (t, C-6), 35.7 (t, C-5), 43.1 (d, C-12), 83.4 (d, C-13), 124.1 (d, C-10), 125.5 (d, C-1), 128.2 (d, C-2), 128.4 (d, C-3), 130.3 (d, C-9), 143.1 (s, C-4).

The signal of C-7 could not be detected.

minor double bond isomer (E isomer, selected signals):

${ }^{13} \mathrm{C} \mathrm{NMR}\left(100 \mathrm{MHz}, \mathrm{CDCl}_{3}\right): \delta=17.9$ (q, C-11), 25.9 (t, C-15), 27.6 (t, C-15'), 125.4 (d, C10), 130.9 (d, C-9).

minor regioisomer (6, mixture of diastereomers, selected signals):

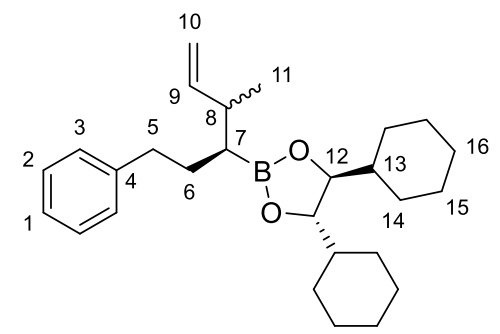

${ }^{1} \mathbf{H}$ NMR (400 MHz, CDCl 3$): \delta=4.88-5.14$ (m, 2 H. 10- $\left.\mathrm{H}_{\mathrm{a}}, 10-\mathrm{H}_{\mathrm{b}}\right), 5.75$ (m, 1 H, 9-H).

HRMS (CI) m/z calcd for $\mathrm{C}_{27} \mathrm{H}_{41} \mathrm{O}_{2} \mathrm{~B}[\mathrm{M}]^{+}:$408.3194, found: 408.3210 .

\section{(S,Z)-1-Phenylhept-5-en-3-ol (5')}

$56 \mathrm{mg}(137 \mu \mathrm{mol})$ boronic ester $5,63.7 \mu \mathrm{l}(33 \%$ in water, $\rho=1.11 \mathrm{~g} / \mathrm{ml}, 686 \mu \mathrm{mol})$ hydrogen peroxide and $27 \mathrm{mg}(686 \mu \mathrm{mol})$ sodium hydroxide were reacted according to GP 2 . After $1.5 \mathrm{~h}$, the reaction was worked up and the crude product was purified by flash chromatography (pentane, diethyl ether 6:4) to yield 5' $\left(E: Z=19: 81\right.$ according to $\left.{ }^{1} \mathrm{H} N M R\right)$ in $84 \%$ yield (22 mg, $116 \mu \mathrm{mol}$ ) as a colorless oil, $\mathrm{R}_{\mathrm{f}}=0.28$ (pentane, diethyl ether 8:2).

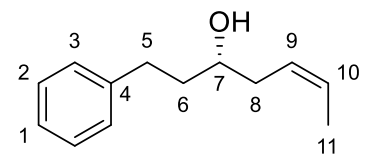

major isomer ( $Z$ isomer):

${ }^{1} \mathbf{H}$ NMR $\left(400 \mathrm{MHz}, \mathrm{CDCl}_{3}\right): \delta=1.63-1.68(\mathrm{~m}, 4 \mathrm{H}, 11-\mathrm{H}, \mathrm{OH}), 1.80(\mathrm{~m}, 2 \mathrm{H}, 6-\mathrm{H}), 2.27$ (m, $2 \mathrm{H}, 8-\mathrm{H}), 2.69\left(\mathrm{~m}, 1 \mathrm{H}, 5-\mathrm{H}_{\mathrm{a}}\right), 2.82\left(\mathrm{~m}, 2 \mathrm{H}, 5-\mathrm{H}_{\mathrm{b}}\right), 3.68(\mathrm{~m}, 1 \mathrm{H}, 7-\mathrm{H}), 5.44(\mathrm{~m}, 1 \mathrm{H}, 9-\mathrm{H})$, $5.67\left(\mathrm{dqt},{ }^{3} J_{10,9}=10.9 \mathrm{~Hz},{ }^{3} J_{10,11}=6.8 \mathrm{~Hz},{ }^{4} J_{10,8}=1.3 \mathrm{~Hz}, 1 \mathrm{H}, 10-\mathrm{H}\right), 7.16-7.25(\mathrm{~m}, 3 \mathrm{H}, 1-\mathrm{H}$, 3-H), 7.30 (m, 2 H, 2-H).

${ }^{13}$ C NMR (100 MHz, CDCl 3 ): $\delta=13.0$ (q, C-11), 32.1 (t, C-5), 35.1 (t, C-8), 38.4 (t, C-6), 70.7 (d, C-7), 125.8 (d, C-1), 125.9 (d, C-9), 127.5 (d, C-10), 128.4 (d, C-2), 128.4 (d, C-3), 142.1 (s, C-4).

minor isomer (E isomer, selected signals):

${ }^{1} \mathbf{H}$ NMR $\left(400 \mathrm{MHz}, \mathrm{CDCl}_{3}\right): \delta=1.70\left(\mathrm{ddt},{ }^{3} J_{11,10}=6.2 \mathrm{~Hz},{ }^{4} J_{11,9} \approx{ }^{5} J_{11,8}=1.0 \mathrm{~Hz}, 3 \mathrm{H}, 11-\mathrm{H}\right)$, $2.11\left(\mathrm{ddd},{ }^{2} J_{8 \mathrm{a}, 8 \mathrm{~b}}=14.8 \mathrm{~Hz},{ }^{3} J_{8 \mathrm{a}, 7} \approx{ }^{3} J_{8 \mathrm{a}, 9}=7.9 \mathrm{~Hz}, 1 \mathrm{H}, 8-\mathrm{H}_{\mathrm{a}}\right), 3.62(\mathrm{~m}, 1 \mathrm{H}, 7-\mathrm{H}), 5.58$ (dqt, $\left.{ }^{3} J_{10,9}=15.2 \mathrm{~Hz},{ }^{3} J_{10,11}=6.4 \mathrm{~Hz},{ }^{4} J_{10,8}=1.1 \mathrm{~Hz}, 1 \mathrm{H}, 10-\mathrm{H}\right)$.

${ }^{13}$ C NMR (100 MHz, CDCl 3 ): $\delta=18.0$ (q, C-11), 32.1 (t, C-5), 38.4 (t, C-6), 40.8 (t, C-8), 70.2 (d, C-7), 126.9 (d, C-9), 129.2 (d, C-10), 142.2 (s, C-4).

HRMS (CI) m/z calcd for $\mathrm{C}_{13} \mathrm{H}_{17} \mathrm{O}[\mathrm{M}-\mathrm{H}]^{+}:$189.1274, found: 189.1281 . 
Allylic bromide 8a was purchased from Sigma-Aldrich, $(\boldsymbol{E}) \mathbf{- 8 g}$ was purchased from Fisher Scientific and $(\boldsymbol{Z})-\mathbf{8} \mathbf{b}^{6},(\boldsymbol{Z})-\mathbf{8} \mathbf{c}^{7},(\boldsymbol{E})-\mathbf{8} \mathbf{d}^{8},(\boldsymbol{E})-\mathbf{8} \mathbf{e}^{9},(\boldsymbol{E})-\mathbf{8} \mathbf{f}^{10}$ and $(\boldsymbol{Z})-\mathbf{8 g ^ { 1 1 }}$ were prepared according to literature procedures.

\section{2-Methallylzinc bromide lithium chloride (9a)}

$252 \mu \mathrm{l}(\rho=1.339 \mathrm{~g} / \mathrm{ml}, 2.50 \mathrm{mmol})$ allylic bromide 8a, $117 \mathrm{mg}(2.75 \mathrm{mmol})$ lithium chloride, $327 \mathrm{mg}(5.00 \mathrm{mmol})$ zinc dust, $4.3 \mu \mathrm{l}(\rho=2.18 \mathrm{~g} / \mathrm{ml}, 50 \mu \mathrm{mol})$ 1,2-dibromoethane and $16 \mu \mathrm{l}$ $(\rho=0.856 \mathrm{~g} / \mathrm{ml}, 125 \mu \mathrm{mol}) \mathrm{TMSCl}$ were reacted according to GP3. After stirring for $3 \mathrm{~h}, \mathbf{9 a}$ was obtained as a $0.23 \mathrm{M}$ solution in THF ( $46 \%$ yield) according to iodometric titration.

\section{Pent-2-en-1-ylzinc bromide lithium chloride (9b)}

$373 \mathrm{mg}(2.50 \mathrm{mmol})$ allylic bromide $(\boldsymbol{Z})-8 \mathbf{b}, 117 \mathrm{mg}(2.75 \mathrm{mmol})$ lithium chloride, $327 \mathrm{mg}$ $(5.00 \mathrm{mmol})$ zinc dust, $4.3 \mu \mathrm{l}(\rho=2.18 \mathrm{~g} / \mathrm{ml}, 50 \mu \mathrm{mol})$ 1,2-dibromoethane and $16 \mu \mathrm{l}(\rho=$ $0.856 \mathrm{~g} / \mathrm{ml}, 125 \mu \mathrm{mol}) \mathrm{TMSCl}$ were reacted according to GP3. After stirring for $2 \mathrm{~h}, \mathbf{9 b}$ was obtained as a $0.42 \mathrm{M}$ solution in THF ( $84 \%$ yield) according to iodometric titration.

\section{Hex-2-en-1-ylzinc bromide lithium chloride (9c)}

$408 \mathrm{mg}(2.50 \mathrm{mmol})$ allylic bromide $(Z)-8 \mathrm{c}, 117 \mathrm{mg}(2.75 \mathrm{mmol})$ lithium chloride, $327 \mathrm{mg}$ $(5.00 \mathrm{mmol})$ zinc dust, $4.3 \mu \mathrm{l}(\rho=2.18 \mathrm{~g} / \mathrm{ml}, 50 \mu \mathrm{mol})$ 1,2-dibromoethane and $16 \mu \mathrm{l}(\rho=$ $0.856 \mathrm{~g} / \mathrm{ml}, 125 \mu \mathrm{mol})$ TMSCl were reacted according to GP3. After stirring for $2 \mathrm{~h}, 9 \mathrm{c}$ was obtained as a $0.43 \mathrm{M}$ solution in THF ( $86 \%$ yield) according to iodometric titration.

\section{Oct-2-en-1-ylzinc bromide lithium chloride (9d)}

$1.02 \mathrm{~g}(5.00 \mathrm{mmol})$ allylic bromide $(\boldsymbol{E})-8 d, 233 \mathrm{mg}(5.50 \mathrm{mmol})$ lithium chloride, $654 \mathrm{mg}$ $(10.0 \mathrm{mmol})$ zinc dust, $8.6 \mu \mathrm{l}(\rho=2.18 \mathrm{~g} / \mathrm{ml}, 100 \mu \mathrm{mol})$ 1,2-dibromoethane and $32 \mu \mathrm{l}(\rho=$ $0.856 \mathrm{~g} / \mathrm{ml}, 250 \mu \mathrm{mol}$ ) $\mathrm{TMSCl}$ were reacted according to GP3. After stirring for $2 \mathrm{~h}, 9 \mathrm{~d}$ was obtained as a $0.39 \mathrm{M}$ solution in THF (78 \% yield) according to iodometric titration.

\footnotetext{
${ }^{6}$ Madelaine, C.; Valerio, V.; Maulide, N., Unexpected Electrophilic Rearrangements of Amides: A Stereoselective Entry to Challenging Substituted Lactones. Angew. Chem. Int. Ed. 2010, 49, 1583-1586.

${ }^{7}$ Wang, H.; Yang, J. C.; Buchwald, S. L., CuH-Catalyzed Regioselective Intramolecular Hydroamination for the Synthesis of Alkyl-Substituted Chiral Aziridines. J. Am. Chem. Soc. 2017, 139, 8428-8431.

${ }^{8}$ Binns, M.; Haynes, R.; Lambert, D.; Schober, P.; Turner, S., The Preparation of Some Octenyl Sulfides From Oct-1-en-3-ol and Oct-2-en-1-ol. Austr. J. Chem. 1987, 40, 281-290.

${ }^{9}$ Seebach, D.; Widler, L., A case of highly diastereoselective addition to unsymmetrical ketones: lk-addition of (2-alkenyl)triphenoxytitanium derivatives. Helv. Chim. Acta 1982, 65, 1972-1981.

${ }^{10}$ Grassi, D.; Alexakis, A., Improvements and Applications of the Transition Metal-Free Asymmetric Allylic Alkylation using Grignard Reagents and Magnesium Alanates. Adv. Synth. Catal. 2015, 357, 3171-3186.

${ }^{11}$ Vyas, D. J.; Oestreich, M., Expedient access to branched allylic silanes by copper-catalysed allylic substitution of linear allylic halides. Chem. Commun. 2010, 46, 568-570.
} 


\section{4-Methylpent-2-en-1-ylzinc bromide lithium chloride (9e)}

$408 \mathrm{mg}(2.50 \mathrm{mmol})$ allylic bromide $(\boldsymbol{E})-8 \mathbf{e}, 117 \mathrm{mg}(2.75 \mathrm{mmol})$ lithium chloride, $327 \mathrm{mg}$ $(5.00 \mathrm{mmol})$ zinc dust, $4.3 \mu \mathrm{l}(\rho=2.18 \mathrm{~g} / \mathrm{ml}, 50 \mu \mathrm{mol})$ 1,2-dibromoethane and $16 \mu \mathrm{l}$ ( $\rho=0.856 \mathrm{~g} / \mathrm{ml}, 125 \mu \mathrm{mol}$ ) TMSCl were reacted according to GP3. After stirring for $2 \mathrm{~h}, \mathbf{9 e}$ was obtained as a $0.33 \mathrm{M}$ solution in THF (66\% yield) according to iodometric titration.

\section{4,4-Dimethylpent-2-en-1-ylzinc bromide lithium chloride (9f)}

$471 \mathrm{mg}(2.50 \mathrm{mmol})$ allylic bromide $(\boldsymbol{E})-8 f, 117 \mathrm{mg}(2.75 \mathrm{mmol})$ lithium chloride, $327 \mathrm{mg}$ $(5.00 \mathrm{mmol})$ zinc dust, $4.3 \mu \mathrm{l}(\rho=2.18 \mathrm{~g} / \mathrm{ml}, 50 \mu \mathrm{mol})$ 1,2-dibromoethane and $16 \mu \mathrm{l}(\rho=$ $0.856 \mathrm{~g} / \mathrm{ml}, 125 \mu \mathrm{mol}$ ) TMSCl were reacted according to GP3. After stirring for $2 \mathrm{~h}, \mathbf{9 f}$ was obtained as a $0.44 \mathrm{M}$ solution in THF ( $88 \%$ yield) according to iodometric titration.

\section{Cinnamylzinc bromide lithium chloride (9g)}

from $(\boldsymbol{E})-8 g$ :

$3.94 \mathrm{~g}$ (20.0 mmol) trans-cinnamyl bromide $(\boldsymbol{E}) \mathbf{- 8 g}, 933 \mathrm{mg}(22.0 \mathrm{mmol})$ lithium chloride, $2.62 \mathrm{~g}(40.0 \mathrm{mmol})$ zinc dust, $34 \mu \mathrm{l}(\rho=2.18 \mathrm{~g} / \mathrm{ml}, 400 \mu \mathrm{mol})$ 1,2-dibromoethane and $128 \mu \mathrm{l}$ ( $\rho=0.856 \mathrm{~g} / \mathrm{ml}, 1.00 \mathrm{mmol}$ ) TMSCl were reacted according to GP3. After stirring for $3 \mathrm{~h}, \mathbf{9 g}$ was obtained as a $0.37 \mathrm{M}$ solution in THF (74 \% yield) according to iodometric titration.

from (Z)-8g:

$493 \mathrm{mg}$ (2.50 mmol) cis-cinnamyl bromide (Z)-8g, $117 \mathrm{mg}(2.75 \mathrm{mmol})$ lithium chloride, $327 \mathrm{mg}(5.00 \mathrm{mmol})$ zinc dust, $4.3 \mu \mathrm{l}(\rho=2.18 \mathrm{~g} / \mathrm{ml}, 50 \mu \mathrm{mol})$ 1,2-dibromoethane and $16 \mu \mathrm{l}$ $(\rho=0.856 \mathrm{~g} / \mathrm{ml}, 125 \mu \mathrm{mol}) \mathrm{TMSCl}$ were reacted according to GP3. After stirring for $2 \mathrm{~h}, \mathbf{9 f}$ was obtained as a $0.39 \mathrm{M}$ solution in THF (78 \% yield) according to iodometric titration.

\section{(4S,5S)-4,5-Dicyclohexyl-2-[(S)-5-methyl-1-phenylhex-5-en-3-yl]-1,3,2-dioxaborolane (10a)}

According to GP $4100 \mathrm{mg}(256 \mu \mathrm{mol}) \alpha$-chloroboronic ester 7 were reacted with $2.23 \mathrm{ml}$ $(513 \mu \mathrm{mol})$ allylzinc reagent $9 \mathrm{a}(0.23 \mathrm{M}$ in THF). After stirring for $19 \mathrm{~h}$, the reaction was worked up and the crude product was purified by flash chromatography (petroleum ether, ethyl acetate $98: 2)$ to give $\mathbf{1 0 a}$ in $85 \%$ yield $(89 \mathrm{mg}, 157 \mu \mathrm{mol})$ as a colorless oil, $\mathrm{R}_{\mathrm{f}}=0.22$ (petroleum ether, ethyl acetate 98:2); $\alpha_{D}^{20}=-18.0\left(\mathrm{c}=1.0, \mathrm{CHCl}_{3}\right)$.

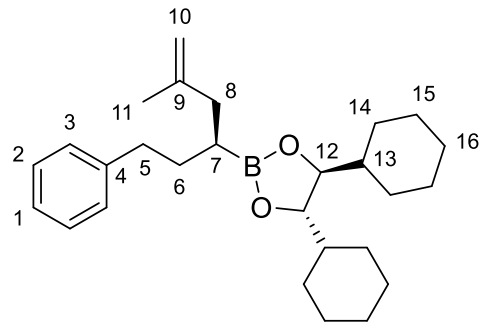

${ }^{1} \mathbf{H}$ NMR $\left(400 \mathrm{MHz}, \mathrm{CDCl}_{3}\right): \delta=0.98(\mathrm{~m}, 2 \mathrm{H}, 15-\mathrm{H}), 1.06(\mathrm{~m}, 2 \mathrm{H}, 14-\mathrm{H}), 1.12-1.38(\mathrm{~m}, 9 \mathrm{H}$, 7-H, 13-H, 14-H', 15-H'), 1.61 (m, 2 H, 14-H' '), 1.65-1.73 (m, 7 H, 6-H, 11-H, 16-H), $1.73-$ $1.88\left(\mathrm{~m}, 6 \mathrm{H}, 14-\mathrm{H}^{\prime},,, 15-\mathrm{H}^{\prime},, 16-\mathrm{H}^{\prime}\right), 2.11\left(\mathrm{dd},{ }^{2} J_{8 \mathrm{a}, 8 \mathrm{~b}}=14.2 \mathrm{~Hz},{ }^{3} J_{8 \mathrm{a}, 7}=7.8 \mathrm{~Hz}, 1 \mathrm{H} .8-\mathrm{H}_{\mathrm{a}}\right)$, $2.22\left(\mathrm{dd},{ }^{2} J_{8 \mathrm{~b}, 8 \mathrm{a}}=14.1 \mathrm{~Hz},{ }^{3} J_{8 \mathrm{~b}, 7}=7.9 \mathrm{~Hz}, 1 \mathrm{H}, 8-\mathrm{H}_{\mathrm{b}}\right), 2.58\left(\mathrm{ddd},{ }^{2} J_{5 \mathrm{a}, 5 \mathrm{~b}}=13.3 \mathrm{~Hz},{ }^{3} J_{5 \mathrm{a}, 6 \mathrm{a}}=\right.$ $\left.9.5 \mathrm{~Hz},{ }^{3} J_{5 \mathrm{a}, 6 \mathrm{~b}}=7.1 \mathrm{~Hz}, 1 \mathrm{H}, 5-\mathrm{H}_{\mathrm{a}}\right), 2.67\left(\mathrm{ddd},{ }^{2} J_{5 \mathrm{~b}, 5 \mathrm{a}}=13.3 \mathrm{~Hz},{ }^{3} J_{5 \mathrm{~b}, 6 \mathrm{~b}}=10.0 \mathrm{~Hz},{ }^{3} J_{5 \mathrm{~b}, 6 \mathrm{a}}=6.2 \mathrm{~Hz}\right.$, $\left.1 \mathrm{H}, 5-\mathrm{H}_{\mathrm{b}}\right), 3.84(\mathrm{~m}, 2 \mathrm{H}, 12-\mathrm{H}), 4.69(\mathrm{~m}, 2 \mathrm{H}, 10-\mathrm{H}), 7.12-7.20(\mathrm{~m}, 3 \mathrm{H}, 1-\mathrm{H}, 3-\mathrm{H}), 7.26$ (m, $2 \mathrm{H}, 2-\mathrm{H})$. 
${ }^{13}$ C NMR (100 MHz, $\left.\mathrm{CDCl}_{3}\right): \delta=21.2$ (bs, C-7), 22.2 (q, C-11), 25.9 (t, C-15), 26.0 (t, C-14), 26.5 (t, C-16), 27.7 (t, C-15'), 28.5 (t, C-14'), 33.1 (t, C-6), 35.6 (t, C-5), 39.5 (t, C-8), 43.1 (d, C-13), 83.5 (d, C-12), 110.7 (t, C-10), 125.5 (d, C-1), 128.2 (d, C-2), 128.4 (d, C-3), 143.1 (s, C-4), 145.4 (s, C-9).

HRMS (CI) $\mathrm{m} / \mathrm{z}$ calcd for $\mathrm{C}_{27} \mathrm{H}_{41} \mathrm{O}_{2} \mathrm{~B}[\mathrm{M}]^{+}:$408.3194, found: 408.3208 .

\section{(4S,5S)-4,5-Dicyclohexyl-2-[(S,Z)-1-phenyloct-5-en-3-yl]-1,3,2-dioxaborolane (10b)}

According to GP $4107 \mathrm{mg}(276 \mu \mathrm{mol}) \alpha$-chloroboronic ester 7 were reacted with $1.31 \mathrm{ml}$ $(551 \mu \mathrm{mol})$ allylzinc reagent $\mathbf{9 b}(0.42 \mathrm{M}$ in THF). After stirring for $18 \mathrm{~h}$, the reaction was worked up and the crude product was purified by flash chromatography (petroleum ether, ethyl acetate $98: 2)$ to give $\mathbf{1 0 b}\left(E: Z=41: 59\right.$ according to ${ }^{1} \mathrm{H}$ NMR) in $82 \%$ yield $(96 \mathrm{mg}, 227 \mu \mathrm{mol})$ as a colorless oil, $\mathrm{R}_{\mathrm{f}}=0.28$ (petroleum ether, ethyl acetate $98: 2$ ).

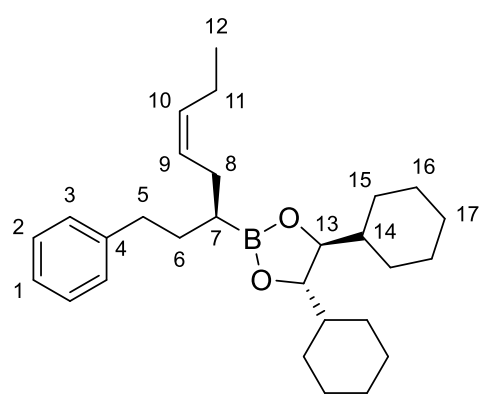

major isomer ( $Z$ isomer):

${ }^{1} \mathbf{H}$ NMR $\left(500 \mathrm{MHz}, \mathrm{CDCl}_{3}\right): \delta=0.93-1.03\left(\mathrm{~m},{ }^{3} J_{12,11}=7.6 \mathrm{~Hz}, 5 \mathrm{H}, 12-\mathrm{H}, 16-\mathrm{H}\right), 1.07(\mathrm{~m}$, $2 \mathrm{H}, 15-\mathrm{H}), 1.11-1.28$ (m, 7 H, 7-H, 15-H', 16-H'), 1.32 (m, $2 \mathrm{H}, 14-\mathrm{H}), 1.62$ (m, $2 \mathrm{H}, 15-\mathrm{H}$ ' '), 1.65-1.71 (m, 3 H, 6- $\left.\mathrm{H}_{\mathrm{a}}, 17-\mathrm{H}\right), 1.71-1.85$ (m, 7 H, 6-Hb, 15-H' ', 16-H', 17-H'), 2.05 (dq, $\left.{ }^{3} J_{11,10} \approx{ }^{3} J_{11,12}=7.2 \mathrm{~Hz}, 2 \mathrm{H}, 11-\mathrm{H}\right), 2.18(\mathrm{~m}, 2 \mathrm{H}, 8-\mathrm{H}), 2.62(\mathrm{~m}, 2 \mathrm{H}, 5-\mathrm{H}), 3.85(\mathrm{~m}, 2 \mathrm{H}$, 13-H), 5.29-5.51 (m, 2 H, 9-H, 10-H), 7.14-7.20 (m, 3 H, 1-H, 3-H), 7.27 (m, 2 H, 2-H).

${ }^{13} \mathrm{C} \mathrm{NMR}\left(125 \mathrm{MHz}, \mathrm{CDCl}_{3}\right): \delta=14.3$ (q, C-12), 20.6 (t, C-11), 23.5 (bs, C-7), 25.9 (t, C-16), 26.0 (t, C-15), 26.5 (t, C-17), 27.6 (t, C-16'), 28.4 (t, C-15'), 28.6 (t, C-8), 33.1 (t, C-6), 35.6 (t, C-5), 43.1 (d, C-14), 83.4 (d, C-13), 125.5 (d, C-1), 128.2 (d, C-2), 128.4 (d, C-3), 128.7 (d, C-9), 132.0 (d, C-10), 143.1 (s, C-4).

minor isomer (E isomer, selected signals):

${ }^{1} \mathbf{H}$ NMR (500 MHz, $\left.\mathrm{CDCl}_{3}\right): \delta=1.98(\mathrm{~m}, 2 \mathrm{H}, 11-\mathrm{H})$.

${ }^{13}$ C NMR (125 MHz, $\left.\mathrm{CDCl}_{3}\right): \delta=13.8$ (q, C-12), 25.5 (t, C-11), 27.6 (t, C-16'), 33.2 (t, C-6), 34.2 (t, C-8), 35.6 (t, C-5), 43.1 (d, C-14), 128.5 (d, C-9), 132.6 (d, C-10), 143.2 (s, C-4).

HRMS (CI) m/z calcd for $\mathrm{C}_{28} \mathrm{H}_{43} \mathrm{O}_{2} \mathrm{~B}[\mathrm{M}]^{+}:$: 422.3351, found: 422.3372 .

\section{(4S,5S)-4,5-Dicyclohexyl-2-[(S,Z)-1-phenylnon-5-en-3-yl]-1,3,2-dioxaborolane (10c)}

According to GP $4100 \mathrm{mg}(256 \mu \mathrm{mol}) \alpha$-chloroboronic ester 7 were reacted with $1.19 \mathrm{ml}$ $(513 \mu \mathrm{mol})$ allylzinc reagent $9 \mathrm{c}(0.43 \mathrm{M}$ in THF). After stirring for $18 \mathrm{~h}$, the reaction was worked up and the crude product was purified by flash chromatography (petroleum ether, ethyl acetate 98:2) to give 10c $\left(E: Z=42: 58\right.$ according to $\left.{ }^{1} \mathrm{H} \mathrm{NMR}\right)$ in $80 \%$ yield $(90 \mathrm{mg}, 206 \mu \mathrm{mol})$ as a colorless oil, $\mathrm{R}_{\mathrm{f}}=0.29$ (petroleum ether, ethyl acetate $98: 2$ ). 


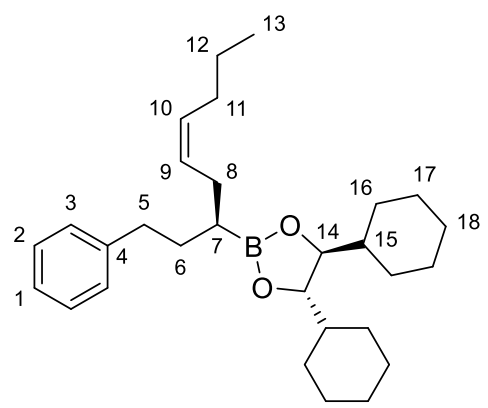

major isomer ( $Z$ isomer):

${ }^{1} \mathbf{H}$ NMR $\left(500 \mathrm{MHz}, \mathrm{CDCl}_{3}\right): \delta=0.88\left(\mathrm{t},{ }^{3} \mathrm{~J}_{13,12}=7.3 \mathrm{~Hz}, 3 \mathrm{H}, 13-\mathrm{H}\right), 1.00(\mathrm{~m}, 2 \mathrm{H}, 17-\mathrm{H}), 1.07$ (m, 2 H, 16-H), 1.11-1.28 (m, 7 H, 7-H, 16-H', 17-H'), 1.28-1.40 (m, 4 H, 12-H, 15-H), $1.52-$ 1.85 (m, 12 H, 6-H, 16-H', 17-H', 18-H), 2.02 (m, 2 H, 11-H), 2.18 (m, 2 H, 8-H), 2.63 (m, $2 \mathrm{H}, 5-\mathrm{H}), 3.85$ (m, $2 \mathrm{H}, 14-\mathrm{H}), 5.33-5.46$ (m, $2 \mathrm{H}, 9-\mathrm{H}, 10-\mathrm{H}), 7.14-7.20$ (m, $3 \mathrm{H}, 1-\mathrm{H}, 3-\mathrm{H})$, 7.27 (m, $2 \mathrm{H}, 2-\mathrm{H})$.

${ }^{13} \mathrm{C}$ NMR (125 MHz, CDCl 3 ): $\delta=13.9$ (q, C-13), 22.9 (t, C-12), 23.5 (bs, C-7), 25.9 (t, C-17), 26.0 (t, C-16), 26.5 (t, C-18), 27.6 (t, C-17'), 28.4 (t, C-16'), 28.7 (t, C-8), 29.4 (t, C-11), 33.1 (t, C-6), 35.7 (t, C-5), 43.1 (d, C-15), 83.4 (d, C-14), 125.5 (d, C-1), 128.2 (d, C-2), 128.4 (d, C-3), 129.5 (d, C-9), 130.2 (d, C-10), 143.1 (s, C-4).

minor isomer (E isomer, selected signals):

${ }^{1} \mathbf{H}$ NMR (500 MHz, $\left.\mathrm{CDCl}_{3}\right): \delta=1.94(\mathrm{~m}, 2 \mathrm{H}, 11-\mathrm{H})$.

${ }^{13}$ C NMR (125 MHz, CDCl 3 ): $\delta=13.7$ (q, C-13), 22.6 (t, C-12), 27.6 (t, C-17'), 34.2 (t, C-8), 34.7 (t, C-11), 35.6 (t, C-5), 43.1 (d, C-15), 129.7 (d, C-9), 130.9 (d, C-10), 143.2 (s, C-4).

HRMS (CI) m/z calcd for $\mathrm{C}_{29} \mathrm{H}_{47} \mathrm{O}_{2} \mathrm{~B}[\mathrm{M}+2 \mathrm{H}]^{+}:$438.3664, found: 438.3687 .

\section{(4S,5S)-4,5-Dicyclohexyl-2-[(S,Z)-1-phenylundec-5-en-3-yl]-1,3,2-dioxaborolane (10d)}

According to GP $4100 \mathrm{mg}(256 \mu \mathrm{mol}) \alpha$-chloroboronic ester 7 were reacted with $1.31 \mathrm{ml}$ $(513 \mu \mathrm{mol})$ allylzinc reagent $9 \mathrm{~d}(0.39 \mathrm{M}$ in THF). After stirring for $18 \mathrm{~h}$, the reaction was worked up and the crude product was purified by flash chromatography (petroleum ether, ethyl acetate 98:2) to give 10d $\left(E: Z=39: 61\right.$ according to ${ }^{1} \mathrm{H}$ NMR $)$ in $81 \%$ yield (97 mg, $\left.209 \mu \mathrm{mol}\right)$ as a colorless oil, $R_{\mathrm{f}}=0.24$ (petroleum ether, ethyl acetate 98:2).

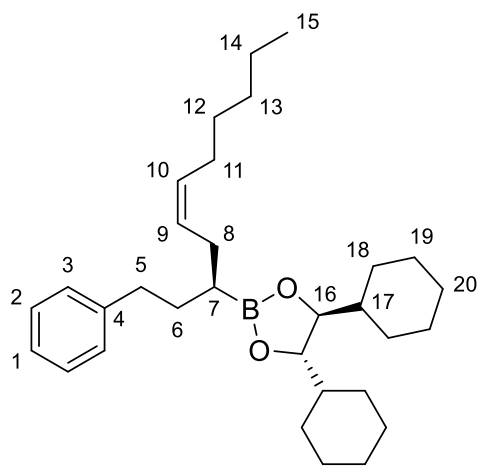

major isomer ( $Z$ isomer):

${ }^{1} \mathbf{H}$ NMR $\left(500 \mathrm{MHz}, \mathrm{CDCl}_{3}\right): \delta=0.88\left(\mathrm{t},{ }^{3} \mathrm{~J}_{15,14}=6.9 \mathrm{~Hz}, 3 \mathrm{H}, 15-\mathrm{H}\right), 0.99(\mathrm{~m}, 2 \mathrm{H}, 19-\mathrm{H}), 1.07$ (m, 2 H, 18-H), 1.11-1.38 (m, 15 H, 7-H, 12-H, 13-H, 14-H, 17-H, 18-H', 19-H'), 1.62 (m, 2 H, 18-H' ), 1.65-1.85 (m, 10 H, 6-H, 18-H', 19-H', 20-H), 2.03 (m, 2 H, 11-H), 2.18 (m, 2 $\mathrm{H}, 8-\mathrm{H}), 2.63(\mathrm{~m}, 2 \mathrm{H}, 5-\mathrm{H}), 3.85(\mathrm{~m}, 2 \mathrm{H}, 16-\mathrm{H}), 5.32-5.46(\mathrm{~m}, 2 \mathrm{H}, 9-\mathrm{H}, 10-\mathrm{H}), 7.13-7.20$ (m, $3 \mathrm{H}, 1-\mathrm{H}, 3-\mathrm{H}), 7.27$ (m, $2 \mathrm{H}, 2-\mathrm{H})$. 
${ }^{13}$ C NMR (125 MHz, $\left.\mathrm{CDCl}_{3}\right): \delta=14.1$ (q, C-15), 22.6 (t, C-14), 23.5 (bs, C-7), 25.9 (t, C-19), 26.0 (t, C-18), 26.5 (t, C-20), 27.3 (t, C-11), 27.6 (t, C-19'), 28.4 (t, C-18'), 28.7 (t, C-8), 29.4 (t, C-12), 31.6 (t, C-13), 33.1 (t, C-6), 35.7 (t, C-5), 43.1 (d, C-17), 83.4 (d, C-16), 125.5 (d, C-1), 128.2 (d, C-2), 128.4 (d, C-3), 129.3 (d, C-9), 130.4 (d, C-10), 143.1 (s, C-4).

minor isomer (E isomer, selected signals):

${ }^{1} \mathbf{H}$ NMR $\left(500 \mathrm{MHz}, \mathrm{CDCl}_{3}\right): \delta=0.88\left(\mathrm{t},{ }^{3} J_{15,14}=6.9 \mathrm{~Hz}, 3 \mathrm{H}, 15-\mathrm{H}\right), 1.96(\mathrm{~m}, 2 \mathrm{H}, 11-\mathrm{H})$.

${ }^{13}$ C NMR (125 MHz, CDCl 3 ): $\delta=22.6$ (t, C-14), 27.6 (t, C-19'), 28.5 (t, C-18'), 29.2 (t, C-12), 31.5 (t, C-13), 32.6 (t, C-11), 33.1 (t, C-6), 34.2 (t, C-8), 35.6 (t, C-5), 43.1 (d, C-17), 129.5 (d, C-9), 131.1 (d, C-10), 143.2 (s, C-4).

HRMS (CI) m/z calcd for $\mathrm{C}_{31} \mathrm{H}_{51} \mathrm{O}_{2} \mathrm{~B}[\mathrm{M}+2 \mathrm{H}]^{+}$: 466.3977, found: 466.3982 .

To confirm that the $(Z)$ isomer was the major isomer, 10d was oxidized to known alcohol 10d'.

\section{(S,Z)-1-Phenylundec-5-en-3-ol (10d')}

$81 \mathrm{mg}(174 \mu \mathrm{mol})$ boronic ester 10d, $81 \mu \mathrm{l}(33 \%$ in water, $\rho=1.11 \mathrm{~g} / \mathrm{ml}, 872 \mu \mathrm{mol})$ hydrogen peroxide and $35 \mathrm{mg}(872 \mu \mathrm{mol})$ sodium hydroxide were reacted according to GP 2 . After $1.5 \mathrm{~h}$, the reaction was worked up and the crude product was purified by flash chromatography (pentane, diethyl ether 8:2) to give 10d' $\left(E: Z=39: 61\right.$ according to $\left.{ }^{1} \mathrm{H} N M R\right)$ in $93 \%$ yield (40 mg, $162 \mu \mathrm{mol}$ ) as a colorless oil, $\mathrm{R}_{\mathrm{f}}=0.20$ (pentane, diethyl ether 8:2). NMR spectra of 10d' were in accordance with previously published data. ${ }^{12}$

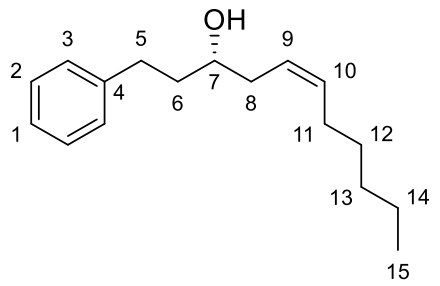

major isomer ( $\mathrm{Z}$ isomer):

${ }^{1} \mathbf{H}$ NMR (500 MHz, $\left.\mathrm{CDCl}_{3}\right): \delta=0.90\left(\mathrm{t},{ }^{3} J_{15,14}=6.9 \mathrm{~Hz}, 3 \mathrm{H}, 15-\mathrm{H}\right), 1.24-1.33(\mathrm{~m}, 4 \mathrm{H}, 13-\mathrm{H}$, 14-H), 1.37 (m, 2 H, 12-H), 1.69 (bs, $1 \mathrm{H}, \mathrm{OH}), 1.80$ (m, $2 \mathrm{H}, 6-\mathrm{H}), 2.05$ (m, $2 \mathrm{H}, 11-\mathrm{H}), 2.27$ (m, $2 \mathrm{H}, 8-\mathrm{H}), 2.70\left(\mathrm{~m}, 1 \mathrm{H}, 5-\mathrm{H}_{\mathrm{a}}\right), 2.82\left(\mathrm{~m}, 1 \mathrm{H}, 5-\mathrm{H}_{\mathrm{b}}\right), 3.65(\mathrm{~m}, 1 \mathrm{H}, 7-\mathrm{H}), 5.41(\mathrm{~m}, 1 \mathrm{H}, 9-\mathrm{H})$, 5.59 (m, $1 \mathrm{H}, 10-\mathrm{H}), 7.17-7.25$ (m, $3 \mathrm{H}, 1-\mathrm{H}, 3-\mathrm{H}), 7.30$ (m, $2 \mathrm{H}, 2-\mathrm{H})$.

${ }^{13} \mathrm{C} \mathrm{NMR}\left(125 \mathrm{MHz}, \mathrm{CDCl}_{3}\right): \delta=14.0$ (q, C-15), 22.5 (t, C-14), 27.4 (t, C-11), 29.3 (t, C-12), 31.5 (t, C-13), 32.1 (t, C-5), 35.4 (t, C-8), 38.4 (t, C-6), 70.7 (d, C-7), 124.8 (d, C-9), 125.7 (d, C-1), 128.3 (d, C-2), 128.4 (d, C-3), 133.8 (d, C-10), 142.1 (s, C-4).

minor isomer (E isomer, selected signals):

${ }^{\mathbf{1}} \mathbf{H}$ NMR $\left(500 \mathrm{MHz}, \mathrm{CDCl}_{3}\right): \delta=2.12\left(\mathrm{ddd},{ }^{2} \boldsymbol{J}_{8 \mathrm{a}, 8 \mathrm{~b}}=13.9 \mathrm{~Hz},{ }^{3} J_{8 \mathrm{a}, 7} \approx{ }^{3} J_{8 \mathrm{a}, 9}=7.9 \mathrm{~Hz}, 1 \mathrm{H}, 8-\mathrm{H}_{\mathrm{a}}\right)$, $2.27\left(\mathrm{~m}, 1 \mathrm{H}, 8-\mathrm{H}_{\mathrm{b}}\right)$.

${ }^{13} \mathrm{C}$ NMR (125 MHz, $\left.\mathrm{CDCl}_{3}\right): \delta=22.5$ (t, C-14), 29.1 (t, C-12), 31.4 (t, C-13), 32.0 (t, C-5), 32.6 (t, C-11), 38.3 (t, C-6), 40.8 (t, C-8), 70.1 (d, C-7), 125.5 (d, C-9), 125.7 (d, C-1), 135.0 (d, C-10), 142.2 (s, C-4).

HRMS (CI) $\mathrm{m} / \mathrm{z}$ calcd for $\mathrm{C}_{17} \mathrm{H}_{27} \mathrm{O}[\mathrm{M}+\mathrm{H}]^{+}:$247.2056, found: 247.2045.

\footnotetext{
${ }^{12}$ Nokami, J.; Nomiyama, K.; Shafi, S. M., A Novel Enantioselective (2Z)-Alk-2-enylation of Aldehydes via an Allyl-Transfer Reaction from Chiral Allyl Donors Prepared from (+)-Isomenthone. Org. Lett. 2004, 6, 12611264.
} 


\section{(4S,5S)-4,5-Dicyclohexyl-2-[(S,E)-7-methyl-1-phenyloct-5-en-3-yl]-1,3,2-dioxaborolane}

(10e)

According to GP $4100 \mathrm{mg}(256 \mu \mathrm{mol}) \alpha$-chloroboronic ester 7 were reacted with $1.51 \mathrm{ml}$ $(513 \mu \mathrm{mol})$ allylzinc reagent $9 \mathrm{e}(0.33 \mathrm{M}$ in THF). After stirring for $18 \mathrm{~h}$, the reaction was worked up and the crude product was purified by flash chromatography (petroleum ether, ethyl acetate 98:2) to give 10e $\left(E: Z=77: 23\right.$ according to $\left.{ }^{1} \mathrm{H} N M R\right)$ in $83 \%$ yield $(93 \mathrm{mg}, 206 \mu \mathrm{mol})$ as a colorless oil, $\mathrm{R}_{\mathrm{f}}=0.21$ (petroleum ether, ethyl acetate 98:2).

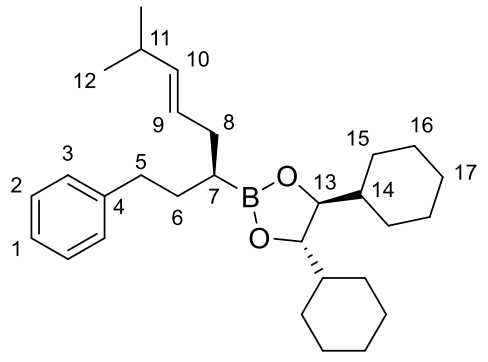

major isomer (E isomer):

${ }^{1} \mathbf{H}$ NMR $\left(500 \mathrm{MHz}, \mathrm{CDCl}_{3}\right): \delta=0.96\left(\mathrm{~d},{ }^{3} J_{12,11}=6.6 \mathrm{~Hz}, 3 \mathrm{H}, 12-\mathrm{H}\right), 0.96\left(\mathrm{~d},{ }^{3} J_{12}, 11=6.6 \mathrm{~Hz}\right.$, 3 H, 12-H'), 1.00 (m, 2 H, 16-H), 1.07 (m, 2 H, 15-H), 1.11-1.28 (m, 7 H, 7-H, 15-H', 16-H'), $1.33(\mathrm{~m}, 2 \mathrm{H}, 14-\mathrm{H}), 1.62\left(\mathrm{~m}, 2 \mathrm{H}, 15-\mathrm{H}^{\prime}\right.$ ') $1.65-1.71\left(\mathrm{~m}, 3 \mathrm{H}, 6-\mathrm{H}_{\mathrm{a}}, 17-\mathrm{H}\right), 1.71-1.85(\mathrm{~m}, 7 \mathrm{H}$, 6-H, $15-\mathrm{H}^{\prime}$ ', $16-\mathrm{H}^{\prime}$ ', 17-H'), 2.08-2.27 (m, $\left.3 \mathrm{H}, 8-\mathrm{H}, 11-\mathrm{H}\right), 2.62$ (m, $\left.2 \mathrm{H}, 5-\mathrm{H}\right), 3.85$ (m, $2 \mathrm{H}$, $13-\mathrm{H}), 5.35\left(\mathrm{dt},{ }^{3} J_{9,10}=15.4 \mathrm{~Hz},{ }^{3} J_{9,8}=6.0 \mathrm{~Hz}, 1 \mathrm{H}, 9-\mathrm{H}\right), 5.42\left(\mathrm{dd},{ }^{3} J_{10,9}=15.4 \mathrm{~Hz},{ }^{3} J_{10,11}=\right.$ $6.0 \mathrm{~Hz}, 1 \mathrm{H}, 10-\mathrm{H}), 7.14-7.20$ (m, $3 \mathrm{H}, 1-\mathrm{H}, 3-\mathrm{H}), 7.27$ (m, $2 \mathrm{H}, 2-\mathrm{H})$.

${ }^{13}$ C NMR (125 MHz, CDCl 3 ): $\delta=22.6$ (q, C-12), 22.6 (q, C-12'), 23.5 (bs, C-7), 25.9 (t, C-16), 26.0 (t, C-15), 26.5 (t, C-17), 27.6 (t, C-16'), 28.5 (t, C-15'), 30.9 (d, C-11), 33.0 (t, C-6), 34.1 (t, C-8), 35.5 (t, C-5), 43.1 (d, C-14), 83.4 (d, C-13), 125.5 (d, C-1), 126.5 (d, C-9), 128.2 (d, C-2), 128.4 (d, C-3), 138.2 (d, C-10), 143.2 (s, C-4).

minor isomer ( $Z$ isomer, selected signals):

${ }^{1} \mathbf{H}$ NMR $\left(500 \mathrm{MHz}, \mathrm{CDCl}_{3}\right): \delta=0.93\left(\mathrm{~d},{ }^{3} J_{12,11}=6.6 \mathrm{~Hz}, 6 \mathrm{H}, 12-\mathrm{H}\right), 2.56-2.69$ (m, $3 \mathrm{H}, 5-\mathrm{H}$, $11-\mathrm{H}), 5.18\left(\mathrm{dd},{ }^{3} J_{10,9}=10.7 \mathrm{~Hz},{ }^{3} J_{10,11}=8.5 \mathrm{~Hz}, 1 \mathrm{H}, 10-\mathrm{H}\right), 5.22\left(\mathrm{dt},{ }^{3} J_{9,10}=10.7 \mathrm{~Hz},{ }^{3} J_{9,8}=\right.$ $6.6 \mathrm{~Hz}, 1 \mathrm{H}, 9-\mathrm{H})$.

${ }^{13}$ C NMR (125 MHz, CDCl 3 ): $\delta=23.2$ (q, C-12), 23.2 (q, C-12'), 27.6 (t, C-16'), 28.8 (t, C-8), 33.1 (t, C-6), 35.6 (t, C-5), 43.1 (d, C-14), 126.9 (d, C-9), 137.9 (d, C-10).

HRMS (CI) m/z calcd for $\mathrm{C}_{29} \mathrm{H}_{47} \mathrm{O}_{2} \mathrm{~B}[\mathrm{M}+2 \mathrm{H}]^{+}$: 438.3664, found: 438.3663 .

\section{$(4 S, 5 S)-4,5-D i c y c l o h e x y l-2-[(S, E)-7,7-d i m e t h y l-1-p h e n y l o c t-5-e n-3-y l]-1,3,2-d i o x a b o r o-$ lane (10f)}

According to GP $4100 \mathrm{mg}(256 \mu \mathrm{mol}) \alpha$-chloroboronic ester 7 were reacted with $1.17 \mathrm{ml}$ $(513 \mu \mathrm{mol})$ allylzinc reagent 9f $(0.44 \mathrm{M}$ in THF). After stirring for $18 \mathrm{~h}$, the reaction was worked up and the crude product was purified by flash chromatography (petroleum ether, ethyl acetate 98:2) to give 10f ( $E: Z>96: 4$ according to ${ }^{1} \mathrm{H}$ NMR $)$ in $81 \%$ yield $(94 \mathrm{mg}, 209 \mu \mathrm{mol})$ as a colorless oil, $\mathrm{R}_{\mathrm{f}}=0.30$ (petroleum ether, ethyl acetate $\left.98: 2\right) ; \alpha_{D}^{20}=-38.0\left(\mathrm{c}=0.5, \mathrm{CHCl}_{3}\right)$. 


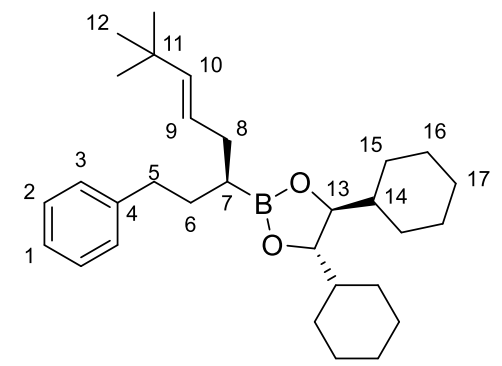

${ }^{1} \mathbf{H}$ NMR (500 MHz, $\left.\mathrm{CDCl}_{3}\right): \delta=0.94-1.04(\mathrm{~m}, 11 \mathrm{H}, 12-\mathrm{H}, 16-\mathrm{H}), 1.07$ (m, $\left.2 \mathrm{H}, 15-\mathrm{H}\right), 1.12-$ 1.27 (m, 7 H, 7-H, 15-H', 16-H'), 1.33 (m, 2 H, 14-H), 1.62 (m, 2 H, 15-H' '), 1.65-1.72 (m, $\left.3 \mathrm{H}, 6-\mathrm{H}_{\mathrm{a}}, 17-\mathrm{H}\right), 1.71-1.86\left(\mathrm{~m}, 7 \mathrm{H}, 6-\mathrm{H}_{\mathrm{b}}, 15-\mathrm{H}^{\prime}{ }^{\prime},{ }^{2}, 16-\mathrm{H}^{\prime},{ }^{2}, 17-\mathrm{H}^{\prime}\right), 2.12\left(\mathrm{ddd},{ }^{2} \mathrm{~J}_{8 \mathrm{a}, 8 \mathrm{~b}}=13.6 \mathrm{~Hz}\right.$, $\left.{ }^{3} J_{8 \mathrm{a}, 7} \approx^{3} J_{8 \mathrm{a}, 9}=6.9 \mathrm{~Hz}, 1 \mathrm{H}, 8-\mathrm{H}_{\mathrm{a}}\right), 2.19\left(\mathrm{ddd},{ }^{2} J_{8 \mathrm{~b}, 8 \mathrm{a}}=13.9 \mathrm{~Hz},{ }^{3} J_{8 \mathrm{a}, 7} \approx^{3} J_{8 \mathrm{a}, 9}=6.9 \mathrm{~Hz}, 1 \mathrm{H}, 8-\mathrm{H}_{\mathrm{b}}\right)$, $2.62(\mathrm{~m}, 2 \mathrm{H}, 5-\mathrm{H}), 3.85(\mathrm{~m}, 2 \mathrm{H}, 13-\mathrm{H}), 5.32\left(\mathrm{dt},{ }^{3} J_{9,10}=15.4 \mathrm{~Hz},{ }^{3} J_{9,8}=6.9 \mathrm{~Hz}, 1 \mathrm{H}, 9-\mathrm{H}\right)$, $5.46\left(\mathrm{dt},{ }^{3} J_{10,9}=15.5 \mathrm{~Hz},{ }^{4} J_{10,8}=1.3 \mathrm{~Hz}, 1 \mathrm{H}, 10-\mathrm{H}\right), 7.14-7.19(\mathrm{~m}, 3 \mathrm{H}, 1-\mathrm{H}, 3-\mathrm{H}), 7.26(\mathrm{~m}$, $2 \mathrm{H}, 2-\mathrm{H})$.

${ }^{13}$ C NMR (125 MHz, CDCl 3 ): $\delta=23.5$ (bs, C-7), 25.9 (t, C-16), 26.0 (t, C-15), 26.5 (t, C-17), 27.6 (t, C-16'), 28.5 (t, C-15'), 29.8 (q, C-12), 32.8 (s, C-11), 32.9 (t, C-6), 34.1 (t, C-8), 35.5 (t, C-5), 43.1 (d, C-14), 83.4 (d, C-13), 124.1 (d, C-9), 125.5 (d, C-1), 128.2 (d, C-2), 128.4 (d, C-3), 142.1 (d, C-10), 143.2 (s, C-4).

The $Z$ isomer could not be detected.

HRMS (CI) m/z calcd for $\mathrm{C}_{30} \mathrm{H}_{48} \mathrm{O}_{2} \mathrm{~B}[\mathrm{M}+\mathrm{H}]^{+}:$451.3742, found: 451.3751 .

\section{(4S,5S)-4,5-Dicyclohexyl-2-[(S,E)-1,6-diphenylhex-5-en-3-yl]-1,3,2-dioxaborolane (10g)}

According to GP $4100 \mathrm{mg}(256 \mu \mathrm{mol}) \alpha$-chloroboronic ester 7 were reacted with $2.23 \mathrm{ml}$ $(513 \mu \mathrm{mol})$ allylzinc reagent $9 \mathrm{~g}(0.23 \mathrm{M}$ in THF, prepared from trans-cinnamyl bromide). After stirring for $18 \mathrm{~h}$, the reaction was worked up and the crude product was purified by flash chromatography (petroleum ether, ethyl acetate 98:2) to give $10 \mathrm{~g}\left(E: Z>96: 4\right.$ according to ${ }^{1} \mathrm{H}$ $\mathrm{NMR})$ in $100 \%$ yield $(120 \mathrm{mg}, 256 \mu \mathrm{mol})$ as a colorless solid, $\mathrm{R}_{\mathrm{f}}=0.15$ (petroleum ether, ethyl acetate 98:2); m.p. $65{ }^{\circ} \mathrm{C}$ (from petroleum ether, ethyl acetate); $\alpha_{D}^{20}=-0.6\left(\mathrm{c}=1.0, \mathrm{CHCl}_{3}\right.$ ). An analytically pure sample of $\mathbf{1 0 g}$ was obtained by reversed-phase flash chromatography $\left(\mathrm{H}_{2} \mathrm{O}, 70 \% \mathrm{MeCN} \rightarrow 100 \% \mathrm{MeCN}\right)$.

If 7 was reacted with $9 \mathrm{~g}$ prepared from cis-cinnamyl bromide under identical conditions, $\mathbf{1 0 g}$ ( $E: Z>96: 4$ according to ${ }^{1} \mathrm{H}$ NMR) was obtained in $90 \%$ yield $(109 \mathrm{mg}, 232 \mu \mathrm{mol})$ as a colorless solid.

If the reaction was performed on a larger scale using $2.28 \mathrm{~g}(5.75 \mathrm{mmol}) \alpha$-chloroboronic ester 7 and $31.1 \mathrm{ml}(11.5 \mathrm{mmol})$ allylzinc reagent $9 \mathrm{~g}(0.37 \mathrm{M}$ in THF, prepared from trans-cinnamyl bromide) under identical conditions, $10 \mathrm{~g}$ ( $E: Z>96: 4$ according to $\left.{ }^{1} \mathrm{H} \mathrm{NMR}\right)$ was obtained in $78 \%$ yield $(2.11 \mathrm{~g}, 4.48 \mathrm{mmol})$ as a pale yellow solid.

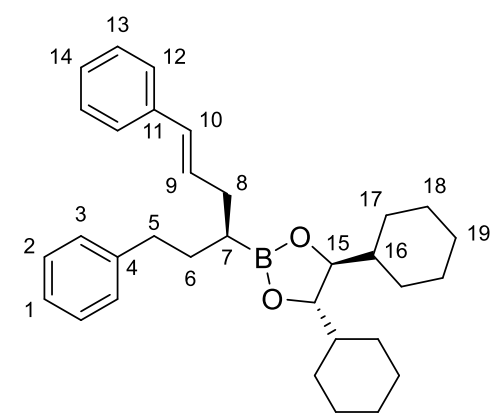


${ }^{1} \mathbf{H}$ NMR $\left(500 \mathrm{MHz}, \mathrm{CDCl}_{3}\right): \delta=0.96(\mathrm{~m}, 2 \mathrm{H}, 18-\mathrm{H}), 1.04(\mathrm{~m}, 2 \mathrm{H}, 17-\mathrm{H}), 1.08-1.22(\mathrm{~m}, 6 \mathrm{H}$, 17-H', 18-H'), 1.24-1.34 (m, 3 H, 7-H, 16-H), 1.55-1.66 (m, 4 H, 17-H', 19-H), 1.66-1.86 (m, 8 H, 6-H, 17-H' ', 18-H', 19-H'), 2.36 (m, 2 H, 8-H), 2.65 (m, 2 H, 5-H), 3.85 (m, 2 H, $15-\mathrm{H}), 6.23\left(\mathrm{dt},{ }^{3} J_{9,10}=15.7 \mathrm{~Hz},{ }^{3} J_{9,8}=7.0 \mathrm{~Hz}, 1 \mathrm{H}, 9-\mathrm{H}\right), 6.38\left(\mathrm{~d},{ }^{3} J_{10,9}=15.9 \mathrm{~Hz}, 1 \mathrm{H}, 10-\mathrm{H}\right)$, 7.13-7.21 (m, 4 H, 1-H, 3-H, 14-H), 7.23-7.29 (m, 4 H, 2-H, 13-H), 7.31 (m, 2 H, 12-H).

${ }^{13} \mathrm{C}$ NMR $\left(125 \mathrm{MHz}, \mathrm{CDCl}_{3}\right): \delta=23.6$ (bs, C-7), 25.8 (t, C-18), $26.0(\mathrm{t}, \mathrm{C}-17), 26.4$ (t, C-19), 27.6 (t, C-18'), 28.5 (t, C-17'), 33.4 (t, C-6), 34.8 (t, C-8), 35.6 (t, C-5), 43.1 (d, C-16), 83.5 (d, C-15), 125.6 (d, C-1), 125.9 (d, C-12), 126.7 (d, C-14), 128.3 (d, C-2), 128.4 (d, C-3), 128.4 (d, C-13), 130.3 (d, C-10), 130.4 (d, C-9), 137.8 (s, C-11), 143.0 (s, C-4).

The $Z$ isomer could not be detected.

HRMS (CI) m/z calcd for $\mathrm{C}_{32} \mathrm{H}_{44} \mathrm{O}_{2} \mathrm{~B}[\mathrm{M}+\mathrm{H}]^{+}:$471.3429, found: 471.3441 .

\section{$(4 S, 5 S)-4,5-D i c y c l o h e x y l-2-[(S, E)-5-m e t h y l-1,6-d i p h e n y l h e x-5-e n-3-y l]-1,3,2-d i o x a b o r o-$ lane (10h)}

According to GP $4100 \mathrm{mg}(256 \mu \mathrm{mol}) \alpha$-chloroboronic ester 7 were reacted with $1.39 \mathrm{ml}$ $(513 \mu \mathrm{mol})$ allylzinc reagent $9 \mathrm{~h}(0.37 \mathrm{M}$ in THF). After stirring for $18 \mathrm{~h}$, the reaction was worked up and the crude product was purified by flash chromatography (petroleum ether, ethyl acetate 98:2) to give 10h $\left(E: Z=86: 14\right.$ according to ${ }^{1} \mathrm{H}$ NMR after oxidation to alcohol 10h') in $85 \%$ yield $(106 \mathrm{mg}, 219 \mu \mathrm{mol})$ as a colorless oil, $\mathrm{R}_{\mathrm{f}}=0.20$ (petroleum ether, ethyl acetate 98:2).

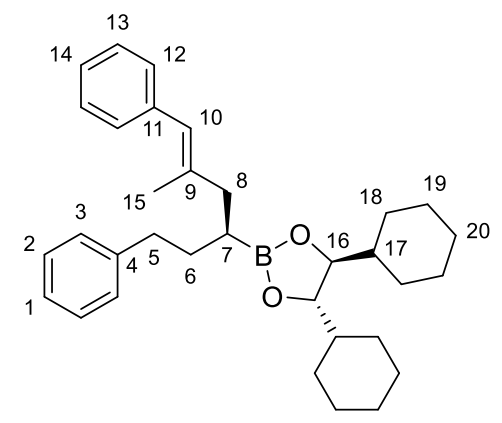

major isomer (E isomer):

${ }^{1} \mathbf{H}$ NMR $\left(400 \mathrm{MHz}, \mathrm{CDCl}_{3}\right): \delta=0.97(\mathrm{~m}, 2 \mathrm{H}, 19-\mathrm{H}), 1.06(\mathrm{~m}, 2 \mathrm{H}, 18-\mathrm{H}), 1.08-1.25(\mathrm{~m}, 6 \mathrm{H}$, 18-H', 19-H'), 1.30 (m, 2 H, 17-H), 1.47 (m, $1 \mathrm{H}, 7-\mathrm{H}), 1.57-1.85$ (m, $12 \mathrm{H}, 6-\mathrm{H}, 18-\mathrm{H}^{\prime}$, $19-\mathrm{H}$ ', $20-\mathrm{H}), 1.86\left(\mathrm{~d},{ }^{4} J_{15,10}=0.9 \mathrm{~Hz}, 3 \mathrm{H}, 15-\mathrm{H}\right), 2.29\left(\mathrm{dd},{ }^{2} J_{8 \mathrm{a}, 8 \mathrm{~b}}=13.6 \mathrm{~Hz},{ }^{3} J_{8 \mathrm{a}, 7}=7.6 \mathrm{~Hz}\right.$, $\left.1 \mathrm{H}, 8-\mathrm{H}_{\mathrm{a}}\right), 2.40\left(\mathrm{dd},{ }^{2} J_{8 \mathrm{~b}, 8 \mathrm{a}}=13.6 \mathrm{~Hz},{ }^{3} J_{8 \mathrm{~b}, 7}=8.4 \mathrm{~Hz}, 1 \mathrm{H}, 8-\mathrm{H}_{\mathrm{b}}\right), 2.68(\mathrm{~m}, 2 \mathrm{H}, 5-\mathrm{H}), 3.86(\mathrm{~m}$, $2 \mathrm{H}, 16-\mathrm{H}), 6.31$ (s, $1 \mathrm{H}, 10-\mathrm{H}), 7.09-7.36$ (m, $10 \mathrm{H}, 1-\mathrm{H}, 2-\mathrm{H}, 3-\mathrm{H}, 12-\mathrm{H}, 13-\mathrm{H}, 14-\mathrm{H})$.

${ }^{13} \mathbf{C ~ N M R}\left(100 \mathrm{MHz}, \mathrm{CDCl}_{3}\right.$ ): $\delta=17.7$ (q, C-15), 21.8 (bs, C-7), 25.8 (t, C-19), 26.0 (t, C-18), 26.4 (t, C-20), 27.7 (t, C-19'), 28.5 (t, C-18'), 33.5 (t, C-6), 35.7 (t, C-5), 42.6 (t, C-8), 43.1 (d, C-17), 83.5 (d, C-16), 125.5 (d, C-1/10), 125.6 (d, C-1/10), 125.7 (d, C-14), 127.9 (d, C-13), 128.2 (d, C-2), 128.4 (d, C-3), 128.9 (d, C-12), 138.6 (s, C-11), 138.6 (s, C-9), 143.0 (s, C-4).

minor isomer ( $Z$ isomer, selected signals):

${ }^{1} \mathbf{H}$ NMR (400 MHz, $\left.\mathrm{CDCl}_{3}\right): \delta=2.35-2.49$ (m, 2 H, 8-H), 3.83 (m, $\left.2 \mathrm{H}, 16-\mathrm{H}\right)$.

${ }^{13} \mathbf{C ~ N M R}\left(100 \mathrm{MHz}, \mathrm{CDCl}_{3}\right): \delta=23.9$ (q, C-15), 25.9 (t, C-19), 26.0 (t, C-18), 26.5 (t, C-20), 27.6 (t, C-19'), 33.1 (t, C-6), 35.6 (t, C-5), 83.5 (d, C-16).

HRMS (CI) m/z calcd for $\mathrm{C}_{33} \mathrm{H}_{45} \mathrm{O}_{2} \mathrm{~B}[\mathrm{M}]^{+}:$484.3507, found: 484.3513 . 


\section{(S,E)-5-Methyl-1,6-diphenylhex-5-en-3-ol (10h')}

$100 \mathrm{mg}(206 \mu \mathrm{mol})$ Boronic ester 10h, $96 \mu \mathrm{l}(33 \%$ in water, $\rho=1.11 \mathrm{~g} / \mathrm{ml}, 1.03 \mathrm{mmol})$ hydrogen peroxide and $41 \mathrm{mg}(1.03 \mathrm{mmol})$ sodium hydroxide were reacted according to GP 2 . After $3 \mathrm{~h}$, the reaction was worked up and the crude product was purified by flash chromatography (pentane, diethyl ether $3: 1)$ to give $\mathbf{1 0 h}$ ' $\left(E: Z=86: 14\right.$ according to $\left.{ }^{1} \mathrm{H} N M R\right)$ in $85 \%$ yield $(47 \mathrm{mg}, 176 \mu \mathrm{mol})$ as a colorless oil, $R_{\mathrm{f}}=0.13$ (pentane, diethyl ether 8:2).

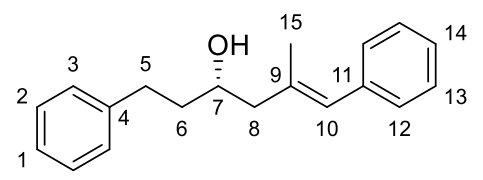

major isomer (E isomer):

${ }^{1} \mathbf{H}$ NMR $\left(500 \mathrm{MHz}, \mathrm{CDCl}_{3}\right): \delta=1.77$ (bs, $\left.1 \mathrm{H}, \mathrm{OH}\right), 1.84(\mathrm{~m}, 2 \mathrm{H}, 6-\mathrm{H}), 1.88\left(\mathrm{~d},{ }^{4} J_{15,10}=\right.$ $1.3 \mathrm{~Hz}, 3 \mathrm{H}, 15-\mathrm{H}), 2.28\left(\mathrm{dd},{ }^{2} J_{8 \mathrm{a}, 8 \mathrm{~b}}=13.2 \mathrm{~Hz},{ }^{3} J_{8,7}=9.1 \mathrm{~Hz}, 1 \mathrm{H}, 8-\mathrm{H}_{\mathrm{a}}\right), 2.39\left(\mathrm{dd},{ }^{2} J_{8 \mathrm{~b}, 8 \mathrm{a}}=\right.$ $\left.13.2 \mathrm{~Hz},{ }^{3} J_{8 \mathrm{~b}, 7}=3.5 \mathrm{~Hz}, 1 \mathrm{H}, 8-\mathrm{H}_{\mathrm{b}}\right), 2.74\left(\mathrm{dt},{ }^{2} J_{5 \mathrm{a}, 5 \mathrm{~b}}=13.9 \mathrm{~Hz},{ }^{3} J_{5 \mathrm{a}, 6}=8.2 \mathrm{~Hz}, 1 \mathrm{H}, 5-\mathrm{H}_{\mathrm{a}}\right), 2.87$ $\left(\mathrm{dt},{ }^{2} J_{5 \mathrm{~b}, 5 \mathrm{a}}=13.9 \mathrm{~Hz},{ }^{3} J_{5 \mathrm{~b}, 6}=7.9 \mathrm{~Hz}, 1 \mathrm{H}, 5-\mathrm{H}_{\mathrm{b}}\right), 3.84(\mathrm{~m}, 1 \mathrm{H}, 7-\mathrm{H}), 6.37$ (s, $\left.1 \mathrm{H}, 10-\mathrm{H}\right), 7.16-$ 7.22 (m, 2 H, 1-H, 14-H), 7.22-7.27 (m, 4 H, 3-H, 3-H, 12-H), 7.27-7.36 (m, 4 H, 2-H, 13-H).

${ }^{13} \mathrm{C}$ NMR (125 MHz, CDCl 3 ): $\delta=18.0$ (q, C-15), 32.1 (t, C-5), 38.8 (t, C-6), $49.1(\mathrm{t}, \mathrm{C}-8), 68.3$ (d, C-7), 125.8 (d, C-1), 126.3 (d, C-14), 128.1 (d, C-13), 128.3 (d, C-10), 128.4 (d, C-2), 128.4 (d, C-3), 128.8 (d, C-12), 135.4 (s, C-9), 137.7 (s, C-11), 142.1 (s, C-4).

minor isomer ( $Z$ isomer, selected signals):

${ }^{1} \mathbf{H}$ NMR $\left(500 \mathrm{MHz}, \mathrm{CDCl}_{3}\right): \delta=1.79(\mathrm{~m}, 2 \mathrm{H}, 6-\mathrm{H}), 1.90\left(\mathrm{~d},{ }^{4} J_{15,10}=1.6 \mathrm{~Hz}, 3 \mathrm{H}, 15-\mathrm{H}\right), 2.25$ $\left(\mathrm{dd},{ }^{2} J_{8 \mathrm{a}, 8 \mathrm{~b}}=13.9 \mathrm{~Hz},{ }^{3} J_{8 \mathrm{a}, 7}=4.1 \mathrm{~Hz}, 1 \mathrm{H}, 8-\mathrm{H}_{\mathrm{a}}\right), 2.62-2.81\left(\mathrm{~m}, 3 \mathrm{H}, 5-\mathrm{H}, 8-\mathrm{H}_{\mathrm{b}}\right), 3.90(\mathrm{~m}, 1 \mathrm{H}$, 7-H), 6.49 (s, $1 \mathrm{H}, 10-\mathrm{H})$.

${ }^{13} \mathrm{C} \mathrm{NMR}\left(125 \mathrm{MHz}, \mathrm{CDCl}_{3}\right): \delta=24.1$ (q, C-15), 32.0 (t, C-5), $38.9(\mathrm{t}, \mathrm{C}-6), 40.2(\mathrm{t}, \mathrm{C}-8), 69.0$ (d, C-7), 126.2 (d, C-14), 128.1 (d, C-13), 128.8 (d, C-10), 129.0 (d, C-12), 135.4 (s, C-9), 137.8 (s, C-11), 142.0 (s, C-4).

HRMS (CI) m/z calcd for $\mathrm{C}_{19} \mathrm{H}_{23} \mathrm{O}[\mathrm{M}+\mathrm{H}]^{+}:$267.1743, found: 267.1761.

\section{(4S,5S)-2-[(R)-1-Chloroethyl]-4,5-dicyclohexyl-1,3,2-dioxaborolane (11)}

(4S,5S)-4,5-Dicyclohexyl-2-methyl-1,3,2-dioxaborolane was prepared as described by Matteson et al. ${ }^{13}$

According to GP 1a $297 \mathrm{mg}$ (1.19 mmol) (4S,5S)-4,5-dicyclohexyl-2-methyl-1,3,2dioxaborolane, $229 \mu \mathrm{l}(\rho=1.32 \mathrm{~g} / \mathrm{ml}, 3.56 \mathrm{mmol})$ anhydrous DCM, $927 \mu \mathrm{l}(1.6 \mathrm{M}$ in hexanes, $1.48 \mathrm{mmol}) n$-butyllithium, $228 \mu \mathrm{l}(\rho=0.71 \mathrm{~g} / \mathrm{ml}, 1.60 \mathrm{mmol})$ diisopropylamine and $324 \mathrm{mg}$ $(2.37 \mathrm{mmol})$ zinc chloride were reacted. After stirring at room temperature for $2 \mathrm{~h}$, the reaction was worked up to obtain crude 11 in $96 \%$ yield (339 mg, $1.13 \mathrm{mmol}$ ) as a pale yellow oil, $\alpha_{D}^{20}$ $=-62.1\left(\mathrm{c}=1.0, \mathrm{CHCl}_{3}\right) .11$ was used in the next step without further purification.

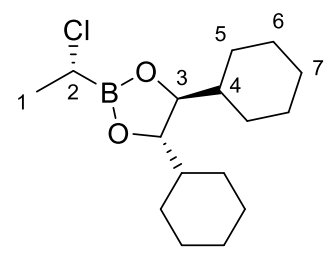

\footnotetext{
${ }^{13}$ Hiscox, W. C.; Matteson, D.S., An Efficient Preparation of $\left(R^{*}, R^{*}\right)$-1,2-Dicyclohexylethane-1,2-diol, a Superior Chiral Director for Synthesis with Boronic Esters. J. Org. Chem. 1996, 61, 8315-8316.
} 
${ }^{1} \mathbf{H}$ NMR $\left(500 \mathrm{MHz}, \mathrm{CDCl}_{3}\right): \delta=0.97(\mathrm{~m}, 2 \mathrm{H}, 6-\mathrm{H}), 1.07(\mathrm{~m}, 2 \mathrm{H}, 5-\mathrm{H}), 1.12-1.30(\mathrm{~m}, 6 \mathrm{H}$, 5-H', 6-H'), 1.38 (m, $2 \mathrm{H}, 4-\mathrm{H}), 1.57$ (d, $\left.{ }^{3} J_{1,2}=7.6 \mathrm{~Hz}, 3 \mathrm{H}, 1-\mathrm{H}\right), 1.60$ (m, $2 \mathrm{H}, 5-\mathrm{H}^{\prime}$ '), 1.68 (m, $2 \mathrm{H}, 7-\mathrm{H}), 1.72-1.81$ (m, $6 \mathrm{H}, 5-\mathrm{H}^{\prime}$ ', $6-\mathrm{H}^{\prime}$ ', 7-H'), 3.56 (q, $\left.{ }^{3} J_{1,2}=7.6 \mathrm{~Hz}, 3 \mathrm{H}, 2-\mathrm{H}\right), 3.95$ (m, $2 \mathrm{H}, 3-\mathrm{H})$.

${ }^{13} \mathrm{C}$ NMR (125 MHz, CDCl 3 ): $\delta=20.7$ (q, C-1), 25.8 (t, C-6), 25.9 (t, C-5), 26.4 (t, C-7), 27.2 (t, C-6'), 28.1 (t, C-5'), 42.8 (d, C-4), 84.1 (d, C-3).

The signal of $\mathrm{C}-2$ could not be detected.

HRMS (CI) $\mathrm{m} / \mathrm{z}$ calcd for $\mathrm{C}_{16} \mathrm{H}_{28} \mathrm{O}_{2} \mathrm{~B}^{37} \mathrm{Cl}[\mathrm{M}]^{+}:$300.1836, found: 300.1829 .

\section{(4S,5S)-2-[(R)-Chloro(cyclohexyl)methyl]-4,5-dicyclohexyl-1,3,2-dioxaborolane (12)}

$(4 S, 5 S)$-2,4,5-tricyclohexyl-1,3,2-dioxaborolane was prepared as described previously. ${ }^{14}$

According to GP 1a $378 \mathrm{mg}(1.18 \mathrm{mmol})(4 S, 5 S)$-2,4,5-tricyclohexyl-1,3,2-dioxaborolane, $229 \mu \mathrm{l}(\rho=1.32 \mathrm{~g} / \mathrm{ml}, 3.56 \mathrm{mmol})$ anhydrous DCM, $928 \mu \mathrm{l}(1.6 \mathrm{M}$ in hexanes, $1.48 \mathrm{mmol})$ $n$-butyllithium, $228 \mu \mathrm{l}(\rho=0.71 \mathrm{~g} / \mathrm{ml}, 1.60 \mathrm{mmol})$ diisopropylamine and $324 \mathrm{mg}(2.38 \mathrm{mmol})$ zinc chloride were reacted. After stirring at room temperature for $2 \mathrm{~h}$, the reaction was worked up to obtain crude 12 in $96 \%$ yield $\left(447 \mathrm{mg}, 1.15 \mathrm{mmol}\right.$ ) as a pale yellow oil, $\alpha_{D}^{20}=-48.3$ $\left(\mathrm{c}=0.5, \mathrm{CHCl}_{3}\right) .12$ was used in the next step without further purification.

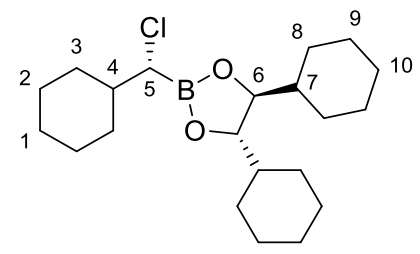

${ }^{1} \mathbf{H}$ NMR (400 MHz, $\left.\mathrm{CDCl}_{3}\right): \delta=0.91-1.32$ (m, $\left.14 \mathrm{H}, 1-\mathrm{H}, 2-\mathrm{H}, 3-\mathrm{H}, 8-\mathrm{H}, 9-\mathrm{H}, 10-\mathrm{H}\right), 1.37$ (m, 2 H, 7-H), 1.54-1.81 (m, 16 H, 1-H', 2-H', 3-H', 4-H, 8-H', 9-H', 10-H'), 1.95 (m, 2 H, $\left.3-\mathrm{H}^{\prime \prime}\right), 3.29$ (d, $\left.{ }^{3} J_{5,4}=7.2 \mathrm{~Hz}, 1 \mathrm{H}, 5-\mathrm{H}\right), 3.94$ (m, $\left.2 \mathrm{H}, 6-\mathrm{H}\right)$.

${ }^{13}$ C NMR (100 MHz, $\left.\mathrm{CDCl}_{3}\right): \delta=25.8(\mathrm{t}, \mathrm{C}-9), 25.9(\mathrm{t}, \mathrm{C}-8, \mathrm{C}-1 / 2), 26.1(\mathrm{t}, \mathrm{C}-1 / 2), 26.2(\mathrm{t}$, C-1/2'), 26.4 (t, C-10), 27.4 (t, C-9'), 28.2 (t, C-8'), 30.5 (t, C-3), 30.9 (t, C-3'), 41.4 (d, C-4), 42.9 (d, C-7), 49.4 (bs, C-5), 84.0 (d, C-6).

HRMS (CI) $\mathrm{m} / \mathrm{z}$ calcd for $\mathrm{C}_{21} \mathrm{H}_{36} \mathrm{O}_{2} \mathrm{~B}[\mathrm{M}-\mathrm{Cl}]^{+}: 331.2803$, found: 331.2801 .

\section{(4S,5S)-2-[(1R,2R)-1-Chloro-2-ethylpentyl]-4,5-dicyclohexyl-1,3,2-dioxaborolane (13)}

$(4 S, 5 S)-4,5$-Dicyclohexyl-2-[(R)-hexan-3-yl]-1,3,2-dioxaborolane was prepared as described previously. ${ }^{14}$

According to GP 1a $504 \mathrm{mg}$ (1.57 mmol) (4S,5S)-4,5-Dicyclohexyl-2-[(R)-hexan-3-yl]-1,3,2dioxaborolane, $304 \mu \mathrm{l}(\rho=1.32 \mathrm{~g} / \mathrm{ml}, 4.72 \mathrm{mmol})$ anhydrous DCM, $1.23 \mathrm{ml}(1.6 \mathrm{M}$ in hexanes, $1.97 \mathrm{mmol}) n$-butyllithium, $303 \mu \mathrm{l}(\rho=0.71 \mathrm{~g} / \mathrm{ml}, 2.12 \mathrm{mmol})$ diisopropylamine and $429 \mathrm{mg}$ (3.15 mmol) zinc chloride were reacted. After stirring at room temperature for $2 \mathrm{~h}$, the reaction was worked up to obtain crude 13 in $97 \%$ yield $(565 \mathrm{mg}, 1.53 \mathrm{mmol})$ as a pale yellow oil, $\alpha_{D}^{20}=-45.4\left(\mathrm{c}=0.5, \mathrm{CHCl}_{3}\right) .13$ was used in the next step without further purification.

\footnotetext{
${ }^{14}$ Andler, O.; Kazmaier, U., A Straightforward Synthesis of Polyketides via Ester Dienolate Matteson Homologation. Chem. Eur. J. 2021, 27, 949-953.
} 


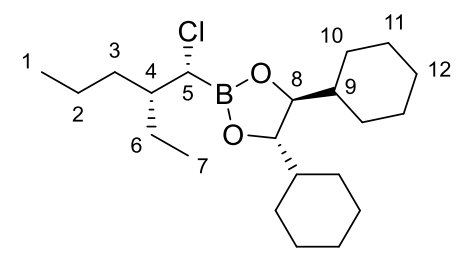

${ }^{1} \mathbf{H}$ NMR $\left(400 \mathrm{MHz}, \mathrm{CDCl}_{3}\right): \delta=0.89\left(\mathrm{t},{ }^{3} J_{7.6}=7.5 \mathrm{~Hz}, 3 \mathrm{H}, 7-\mathrm{H}\right), 0.90\left(\mathrm{t},{ }^{3} J_{1,2}=7.1 \mathrm{~Hz}, 3 \mathrm{H}\right.$, 1-H), 0.95-1.11 (m, 4 H, 10-H, 11-H), 1.12-1.44 (m, 12 H, 2-H, 3-H, 9-H, 10-H', 11-H'), 1.48 (m, $2 \mathrm{H}, 6-\mathrm{H}), 1.61$ (m, $2 \mathrm{H}, 10-\mathrm{H}^{\prime}$ '), 1.68 (m, $\left.2 \mathrm{H}, 12-\mathrm{H}\right), 1.71-1.83$ (m, 7 H, 4-H, 10-H' ', 11-H', 12-H'), 2.13 (m, 2 H, 6-H), 3.57 (d, $\left.{ }^{3} J_{5,4}=5.7 \mathrm{~Hz}, 1 \mathrm{H}, 5-\mathrm{H}\right), 3.93(\mathrm{~m}, 2 \mathrm{H}, 8-\mathrm{H})$.

${ }^{13} \mathrm{C} \mathrm{NMR}\left(100 \mathrm{MHz}, \mathrm{CDCl}_{3}\right): \delta=11.0$ (q, C-7), 14.3 (q, C-1), 20.2 (t, C-2), 24.1 (t, C-6), 25.8 (t, C-11), 25.9 (t, C-10), 26.4 (t, C-12), 27.5 (t, C-11'), 28.3 (t, C-10'), 33.1 (t, C-3), 42.7 (d, C-4), 42.9 (d, C-9), 47.4 (bs, C-5), 84.2 (d, C-8).

HRMS (CI) $\mathrm{m} / \mathrm{z}$ calcd for $\mathrm{C}_{21} \mathrm{H}_{38} \mathrm{O}_{2} \mathrm{~B}$ [M-Cl] $]^{+}: 333.2959$, found: 333.2950 .

\section{(4S,5S)-4,5-Dicyclohexyl-2-[(S,Z)-hex-4-en-2-yl]-1,3,2-dioxaborolane (14c)}

According to GP $4105 \mathrm{mg}(350 \mu \mathrm{mol}) \alpha$-chloroboronic ester 11 were reacted with $1.63 \mathrm{ml}$ $(700 \mu \mathrm{mol})$ allylzinc reagent $4 \mathrm{c}(0.43 \mathrm{M}$ in THF). After stirring for $18 \mathrm{~h}$, the reaction was worked up and the crude product was purified by flash chromatography (petroleum ether, ethyl acetate 98:2) to give 14c ( $E: Z=24: 76$ according to ${ }^{1} \mathrm{H} N M R$ after oxidation to alcohol 17c) in $77 \%$ yield $\left(86 \mathrm{mg}, 270 \mu \mathrm{mol}\right.$ ) as a colorless oil, $R_{\mathrm{f}}=0.27$ (petroleum ether, ethyl acetate 98:2).

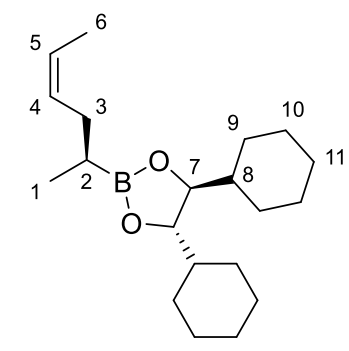

major isomer ( $Z$ isomer):

${ }^{1} \mathbf{H}$ NMR $\left(500 \mathrm{MHz}, \mathrm{CDCl}_{3}\right): \delta=0.94(\mathrm{~m}, 2 \mathrm{H}, 10-\mathrm{H}), 0.99\left(\mathrm{~d},{ }^{3} \mathrm{~J}_{1,2}=7.6 \mathrm{~Hz}, 3 \mathrm{H}, 1-\mathrm{H}\right), 1.05$ (m, 2 H, 9-H), 1.08-1.26 (m, 7 H, 2-H, 9-H', 10-H'), 1.31 (m, $2 \mathrm{H}, 8-\mathrm{H}), 1.56-1.64$ (m, $5 \mathrm{H}$, 6-H, 9-H' '), 1.67 (m, $2 \mathrm{H}, 11-\mathrm{H}), 1.72-1.80$ (m, $6 \mathrm{H}, 9-\mathrm{H}^{\prime}$ ', $\left., 10-\mathrm{H}^{\prime},, 11-\mathrm{H}^{\prime}\right), 2.08$ (ddd, ${ }^{2} J_{3 \mathrm{a}, 3 \mathrm{~b}}=$ $\left.14.4 \mathrm{~Hz},{ }^{3} J_{3 \mathrm{a}, 2} \approx{ }^{3} J_{3 \mathrm{a}, 4}=7.4 \mathrm{~Hz}, 1 \mathrm{H}, 3-\mathrm{H}_{\mathrm{a}}\right), 2.19\left(\mathrm{ddd},{ }^{2} J_{3 \mathrm{~b}, 3 \mathrm{a}}=13.6 \mathrm{~Hz},{ }^{3} J_{3 \mathrm{~b}, 2} \approx{ }^{3} J_{3 \mathrm{~b}, 4}=6.9 \mathrm{~Hz}\right.$, $\left.1 \mathrm{H}, 3-\mathrm{H}_{\mathrm{b}}\right), 3.83$ (m, $\left.2 \mathrm{H}, 7-\mathrm{H}\right), 5.36-5.50$ (m, $\left.2 \mathrm{H}, 4-\mathrm{H}, 5-\mathrm{H}\right)$.

${ }^{13} \mathrm{C} \mathrm{NMR}\left(125 \mathrm{MHz}, \mathrm{CDCl}_{3}\right): \delta=12.9$ (q, C-6), 15.4 (q, C-1), 25.9 (t, C-10), 26.0 (t, C-9), 26.5 (t, C-11), 27.3 (t, C-10'), 28.2 (t, C-9'), 30.2 (t, C-3), 43.0 (d, C-8), 83.2 (d, C-7), 124.0 (d, C-5), 130.5 (d, C-4).

The signal of C-2 could not be detected.

minor isomer ( $E$ isomer, selected signals):

${ }^{13} \mathrm{C}$ NMR (125 MHz, $\mathrm{CDCl}_{3}$ ): $\delta=15.4$ (q, C-1), 17.9 (q, C-6), 25.9 (t, C-10), 27.4 (t, C-10'), 83.2 (d, C-7), 125.2 (d, C-5), 131.1 (d, C-4).

HRMS (CI) m/z calcd for $\mathrm{C}_{20} \mathrm{H}_{35} \mathrm{O}_{2} \mathrm{~B}[\mathrm{M}]^{+}:$318.2725, found: 318.2727. 


\section{(4S,5S)-4,5-Dicyclohexyl-2-[(S,Z)-dec-4-en-2-yl]-1,3,2-dioxaborolane (14d)}

According to GP $4105 \mathrm{mg}(350 \mu \mathrm{mol}) \alpha$-chloroboronic ester 11 were reacted with $1.75 \mathrm{ml}$ $(700 \mu \mathrm{mol})$ allylzinc reagent $9 d(0.40 \mathrm{M}$ in THF). After stirring for $18 \mathrm{~h}$, the reaction was worked up and the crude product was purified by flash chromatography (petroleum ether, ethyl acetate 98:2) to give 14d (E:Z=37:63 according to ${ }^{1} \mathrm{H}$ NMR after oxidation to alcohol 17d) in $91 \%$ yield $(119 \mathrm{mg}, 318 \mu \mathrm{mol})$ as a colorless oil, $\mathrm{R}_{\mathrm{f}}=0.26$ (petroleum ether, ethyl acetate 98:2).

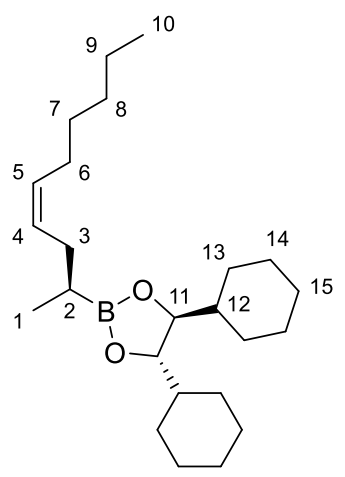

major isomer ( $\mathrm{Z}$ isomer):

${ }^{1} \mathbf{H}$ NMR $\left(500 \mathrm{MHz}, \mathrm{CDCl}_{3}\right): \delta=0.88\left(\mathrm{q},{ }^{3} J_{10,9}=6.8 \mathrm{~Hz}, 3 \mathrm{H}, 10-\mathrm{H}\right), 0.91-1.00\left(\mathrm{~m},{ }^{3} J_{1,2}=\right.$ $7.2 \mathrm{~Hz}, 5 \mathrm{H}, 1-\mathrm{H}, 14-\mathrm{H}), 1.05$ (m, $2 \mathrm{H}, 13-\mathrm{H}), 1.09-1.38$ (m, $15 \mathrm{H}, 2-\mathrm{H}, 7-\mathrm{H}, 8-\mathrm{H}, 9-\mathrm{H}, 12-\mathrm{H}$, 13-H', 14-H'), 1.58 (m, 2 H, 13-H' '), 1.68 (m, 2 H, 15-H), 1.71-1.82 (m, 6 H, 13-H', , 14-H', 15-H'), 1.99-2.11 (m, $\left.3 \mathrm{H}, 3-\mathrm{H}_{\mathrm{a}}, 6-\mathrm{H}\right), 2.17$ (m, $\left.1 \mathrm{H}, 3-\mathrm{H}_{\mathrm{b}}\right), 3.83$ (m, $\left.2 \mathrm{H}, 11-\mathrm{H}\right), 5.31-5.46$ (m, $2 \mathrm{H}, 4-\mathrm{H}, 5-\mathrm{H})$.

${ }^{13} \mathrm{C}$ NMR (125 MHz, CDCl 3 ): $\delta=14.1$ (q, C-10), 15.4 (q, C-1), 16.8 (bs, C-2), 22.6 (t, C-9), 25.9 (t, C-14), 26.0 (t, C-13), 26.5 (t, C-15), 27.2 (t, C-6), 27.4 (t, C-14'), 28.2 (t, C-13'), 29.5 (t, C-7), 30.6 (t, C-3), 31.6 (t, C-8), 43.0 (d, C-12), 83.2 (d, C-11), 129.5 (d, C-4), 130.4 (d, C-5).

minor isomer (E isomer, selected signals):

${ }^{1} \mathbf{H}$ NMR $\left(500 \mathrm{MHz}, \mathrm{CDCl}_{3}\right): \delta=1.96$ (m, $\left.2 \mathrm{H}, 6-\mathrm{H}\right)$.

${ }^{13}$ C NMR (125 MHz, CDCl 3 ): $\delta=15.4$ (q, C-1), 22.6 (t, C-9), 29.3 (t, C-7), 31.4 (t, C-8), 32.6 (t, C-6), 36.2 (t, C-3), 129.7 (d, C-4), 131.0 (d, C-5).

HRMS (CI) m/z calcd for $\mathrm{C}_{24} \mathrm{H}_{44} \mathrm{O}_{2} \mathrm{~B}[\mathrm{M}+\mathrm{H}]^{+}: 375.3429$, found: 375.3431 .

\section{(4S,5S)-4,5-Dicyclohexyl-2-[(R,Z)-1-cyclohexylpent-3-en-1-yl]-1,3,2-dioxaborolane (15c)}

According to GP $4128 \mathrm{mg}$ ( $350 \mu \mathrm{mol}) \alpha$-chloroboronic ester 12 were reacted with $1.63 \mathrm{ml}$ $(700 \mu \mathrm{mol})$ allylzinc reagent $4 \mathrm{c}(0.43 \mathrm{M}$ in THF). After stirring for $18 \mathrm{~h}$, the reaction was worked up and the crude product was purified by flash chromatography (petroleum ether, ethyl acetate 98:2) to give 15c $\left(E: Z=39: 61\right.$ according to ${ }^{1} \mathrm{H}$ NMR after oxidation to alcohol 18c $)$ in $92 \%$ yield $(124 \mathrm{mg}, 321 \mu \mathrm{mol})$ as a colorless oil, $\mathrm{R}_{\mathrm{f}}=0.32$ (petroleum ether, ethyl acetate 98:2).

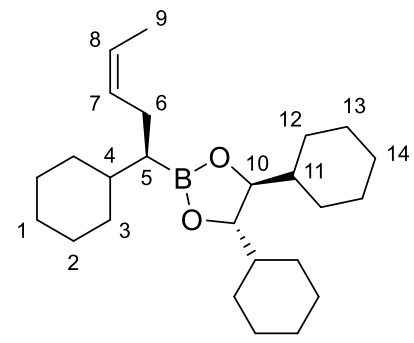


major isomer ( $Z$ isomer):

${ }^{1} \mathbf{H}$ NMR $\left(500 \mathrm{MHz}, \mathrm{CDCl}_{3}\right): \delta=0.90-1.24$ (m, $\left.16 \mathrm{H}, 1-\mathrm{H}, 2-\mathrm{H}, 3-\mathrm{H}, 5-\mathrm{H}, 12-\mathrm{H}, 13-\mathrm{H}\right), 1.28$ (m, 2 H, 11-H), 1.38 (m, $1 \mathrm{H}, 4-\mathrm{H}), 1.56-1.63$ (m, $\left.5 \mathrm{H}, 9-\mathrm{H}, 12-\mathrm{H}^{\prime}\right), 1.63-1.72$ (m, $6 \mathrm{H}, 1-\mathrm{H}^{\prime}$, 2-H', 3-H', 14-H), 1.72-1.83 (m, 7 H, 1-H', 2-H', 3-H', 12-H', 13-H', 14-H'), 2.15 (dd, $\left.{ }^{3} J_{6,5} \approx^{3} J_{6,7}=7.6 \mathrm{~Hz}, 2 \mathrm{H}, 6-\mathrm{H}\right), 3.79(\mathrm{~m}, 2 \mathrm{H}, 10-\mathrm{H}), 5.33-5.45(\mathrm{~m}, 2 \mathrm{H}, 7-\mathrm{H}, 8-\mathrm{H})$.

${ }^{13} \mathrm{C}$ NMR (125 MHz, CDCl 3 ): $\delta=12.8$ (q, C-9), 25.9 (t, C-13), 26.0 (t, C-12), 26.0 (t, C-6), 26.5 (t, C-14), 26.8 (t, C-1, C-2, C-2'), 27.8 (t, C-13'), 28.5 (t, C-12'), 32.1 (t, C-3), 32.8 (t, C-3'), 39.2 (d, C-4), 43.1 (d, C-11), 83.3 (d, C-10), 123.6 (d, C-8), 131.0 (d, C-7).

The signal of C-5 could not be detected.

minor isomer (E isomer, selected signals):

${ }^{1} \mathbf{H}$ NMR $\left(500 \mathrm{MHz}, \mathrm{CDCl}_{3}\right): \delta=2.07$ (m, 2 H. 6-H).

${ }^{13}$ C NMR (125 MHz, CDCl 3 ): $\delta=18.0$ (q, C-9), 27.8 (t, C-13'), 28.6 (t, C-12'), 31.8 (t, C-6), 32.2 (t, C-3), 32.8 (t, C-3'), 39.3 (d, C-4), 43.1 (d, C-11), 83.3 (d, C-10), 124.7 (d, C-8), 131.7 (d, C-7).

HRMS (CI) m/z calcd for $\mathrm{C}_{25} \mathrm{H}_{44} \mathrm{O}_{2} \mathrm{~B}[\mathrm{M}+\mathrm{H}]^{+}: 387.3429$, found: 387.3426 .

\section{(4S,5S)-4,5-Dicyclohexyl-2-[(R,E)-1-cyclohexylnon-3-en-1-yl]-1,3,2-dioxaborolane (15d)}

According to GP $4102 \mathrm{mg}(277 \mu \mathrm{mol}) \alpha$-chloroboronic ester 12 were reacted with $1.38 \mathrm{ml}$ $(554 \mu \mathrm{mol})$ allylzinc reagent $9 \mathbf{d}(0.40 \mathrm{M}$ in THF $)$. After stirring for $18 \mathrm{~h}$, the reaction was worked up and the crude product was purified by flash chromatography (petroleum ether, ethyl acetate 98:2) to give 15d ( $E: Z=59: 41$ according to GC-FID after oxidation to alcohol 18d) in $89 \%$ yield $(109 \mathrm{mg}, 246 \mu \mathrm{mol})$ as a colorless oil, $\mathrm{R}_{\mathrm{f}}=0.28$ (petroleum ether, ethyl acetate 98:2).

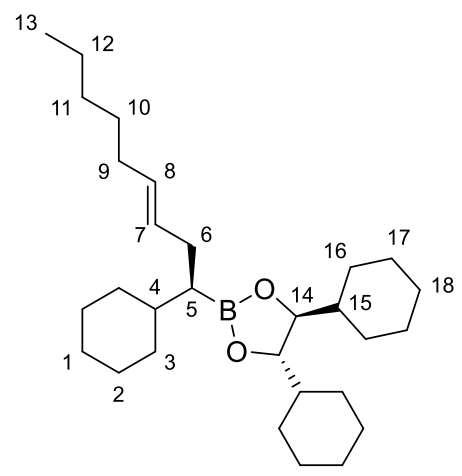

major isomer (E isomer):

${ }^{1} \mathbf{H}$ NMR $\left(400 \mathrm{MHz}, \mathrm{CDCl}_{3}\right): \delta=0.81-1.45$ (m, $28 \mathrm{H}, 1-\mathrm{H}, 2-\mathrm{H}, 3-\mathrm{H}, 4-\mathrm{H}, 5-\mathrm{H}, 10-\mathrm{H}, 11-\mathrm{H}$, 12-H, 13-H, 15-H, 16-H, 17-H), 1.54-1.85 (m, 15 H, 1-H', 2-H', 3-H', 16-H', 17-H', 18-H), 1.93 (m, 2 H, 6-H), 2.10 (m, 2 H, 9-H), 3.79 (m, 2 H, 14-H), 5.36-5.45 (m, 2 H, 7-H, 8-H).

${ }^{13} \mathrm{C}$ NMR (100 MHz, $\left.\mathrm{CDCl}_{3}\right): \delta=14.1$ (q, C-13), 22.6 (t, C-12), 25.9 (t, C-17), 26.0 (t, C-16), 26.5 (t, C-18), 26.7 (t, C-1/2), 26.8 (t, C-1/2), 26.8 (t, C-1/2'), 27.8 (t, C-17'), 28.6 (t, C-16'), 29.3 (t, C-10), 30.8 (bs, C-5), 31.5 (t, C-11), 31.7 (t, C-9), 32.1 (t, C-3), 32.6 (t, C-6), 32.8 (t, C-3'), 39.1 (d, C-4), 43.1 (d, C-15), 83.3 (d, C-14), 130.2 (d, C-7), 130.5 (d, C-8). 
minor isomer ( $Z$ isomer, selected signals):

${ }^{1} \mathbf{H}$ NMR $\left(400 \mathrm{MHz}, \mathrm{CDCl}_{3}\right): \delta=2.02\left(\mathrm{dt},{ }^{3} J_{9,8} \approx^{3} J_{9,10}=6.0 \mathrm{~Hz}, 2 \mathrm{H}, 9-\mathrm{H}\right), 2.14(\mathrm{~m}, 2 \mathrm{H}, 6-\mathrm{H})$, 5.27-5.35 (m, 2 H, 7-H, 8-H).

${ }^{13} \mathrm{C}$ NMR (100 MHz, CDCl 3 ): $\delta=22.6$ (d, C-12), 26.3 (t, C-6), 27.2 (t, C-9), 27.8 (t, C-17'), 28.5 (t, C-16'), 29.5 (t, C-10), 31.6 (t, C-11), 32.8 (t, C-3'), 39.2 (d, C-4), 43.1 (d, C-15), 83.3 (d, C-14), 129.9 (t, C-8), 130.0 (t, C-7).

HRMS (CI) m/z calcd for $\mathrm{C}_{29} \mathrm{H}_{52} \mathrm{O}_{2} \mathrm{~B}[\mathrm{M}+\mathrm{H}]^{+}:$443.4055, found: 443.4077 .

\section{(4S,5S)-4,5-Dicyclohexyl-2-[(R,E)-1-cyclohexyl-5-methylhex-3-en-1-yl]-1,3,2- dioxaborolane (15e)}

According to GP $466 \mathrm{mg}(169 \mu \mathrm{mol}) \alpha$-chloroboronic ester 12 were reacted with $1.30 \mathrm{ml}$ $(338 \mu \mathrm{mol})$ allylzinc reagent $9 \mathrm{e}(0.26 \mathrm{M}$ in THF). After stirring for $18 \mathrm{~h}$, the reaction was worked up and the crude product was purified by flash chromatography (petroleum ether, ethyl acetate $98: 2)$ to give 15e $\left(E: Z=80: 20\right.$ according to $\left.{ }^{1} \mathrm{H} \mathrm{NMR}\right)$ in $34 \%$ yield $(24 \mathrm{mg}, 58 \mu \mathrm{mol})$ as a colorless oil, $R_{\mathrm{f}}=0.30$ (petroleum ether, ethyl acetate $98: 2$ ).

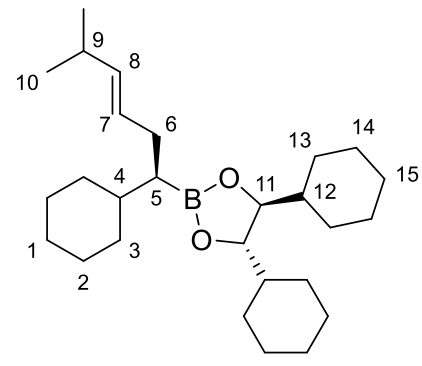

major isomer (E isomer):

${ }^{1} \mathbf{H}$ NMR $\left(500 \mathrm{MHz}, \mathrm{CDCl}_{3}\right): \delta=0.90-1.43\left(\mathrm{~m},{ }^{3} J_{10,9}=6.6 \mathrm{~Hz},{ }^{3} J_{10}, 9=6.9 \mathrm{~Hz}, 25 \mathrm{H}, 1-\mathrm{H}, 2-\mathrm{H}\right.$, 3-H, 4-H, 5-H, 10-H, 10-H', 12-H, 13-H, 14-H), 1.54-1.85 (m, 15 H, 1-H', 2-H', 3-H', 13-H', 14-H', 15-H), 2.09 (dd, $\left.{ }^{3} J_{6,5} \approx^{3} J_{6,7}=6.8 \mathrm{~Hz}, 2 \mathrm{H}, 6-\mathrm{H}\right), 2.19(\mathrm{~m}, 1 \mathrm{H}, 9-\mathrm{H}), 3.79$ (m, $2 \mathrm{H}, 11-$ $\mathrm{H}), 5.33\left(\mathrm{dt},{ }^{3} J_{7,8}=15.4 \mathrm{~Hz},{ }^{3} J_{7,6}=6.0 \mathrm{~Hz}, 1 \mathrm{H}, 7-\mathrm{H}\right), 5.38\left(\mathrm{dd},{ }^{3} J_{8,7}=15.4 \mathrm{~Hz},{ }^{3} J_{8,9}=5.7 \mathrm{~Hz}\right.$, $1 \mathrm{H}, 8-\mathrm{H})$.

${ }^{13}$ C NMR (125 MHz, $\mathrm{CDCl}_{3}$ ): $\delta=22.5$ (q, C-10), 22.6 (q, C-10'), 25.9 (t, C-14), 26.0 (t, C-13), 26.5 (t, C-15), 26.7 (t, C-1/2), 26.8 (t, C-1/2), 26.8 (t, C-1/2'), 27.9 (t, C-14'), 28.6 (t, C-13'), 30.9 (d, C-9), 30.9 (bs, C-5), 31.7 (t, C-6), 32.1 (t, C-3), 32.8 (t, C-3'), 39.2 (d, C-4), 43.2 (d, C-12), 83.3 (d, C-11), 127.2 (d, C-7), 137.5 (d, C-8).

minor isomer ( $Z$ isomer, selected signals):

${ }^{1} \mathbf{H}$ NMR $\left(500 \mathrm{MHz}, \mathrm{CDCl}_{3}\right): \delta=5.13\left(\mathrm{dd},{ }^{3} J_{8,7}=10.4 \mathrm{~Hz},{ }^{3} J_{8,9}=9.5 \mathrm{~Hz}, 1 \mathrm{H}, 8-\mathrm{H}\right), 5.20$ (dt, $\left.{ }^{3} J_{7,8}=10.6 \mathrm{~Hz},{ }^{3} J_{7,6}=7.0 \mathrm{~Hz}, 1 \mathrm{H}, 7-\mathrm{H}\right)$.

${ }^{13}$ C NMR (125 MHz, CDCl $): \delta=23.2$ (q, C-10), 23.3 (q, C-10'), 26.4 (d, C-9), 26.5 (d, C-6), 27.8 (t, C-14'), 32.1 (t, C-3), 32.9 (t, C-3'), 39.2 (d, C-4), 43.1 (d, C-12), 127.7 (d, C-7), 137.4 (d, C-8).

HRMS (CI) m/z calcd for $\mathrm{C}_{27} \mathrm{H}_{48} \mathrm{O}_{2} \mathrm{~B}[\mathrm{M}+\mathrm{H}]^{+}:$415.3742, found: 415.3746 . 


\section{(4S,5S)-4,5-Dicyclohexyl-2-[(5R,6R,Z)-6-ethylnon-2-en-5-yl]-1,3,2-dioxaborolane (16c)}

According to GP $4108 \mathrm{mg}(293 \mu \mathrm{mol}) \alpha$-chloroboronic ester 13 were reacted with $1.50 \mathrm{ml}$ $(586 \mu \mathrm{mol})$ allylzinc reagent $4 \mathrm{c}(0.39 \mathrm{M}$ in $\mathrm{THF})$. After stirring for $18 \mathrm{~h}$, the reaction was worked up and the crude product was purified by flash chromatography (petroleum ether, ethyl acetate 98:2) to give 16c $\left(E: Z=37: 63\right.$ according to ${ }^{1} \mathrm{H}$ NMR after oxidation to alcohol 19c) in $70 \%$ yield ( $80 \mathrm{mg}, 206 \mu \mathrm{mol}$ ) as a colorless oil, $R_{\mathrm{f}}=0.31$ (petroleum ether, ethyl acetate 98:2).

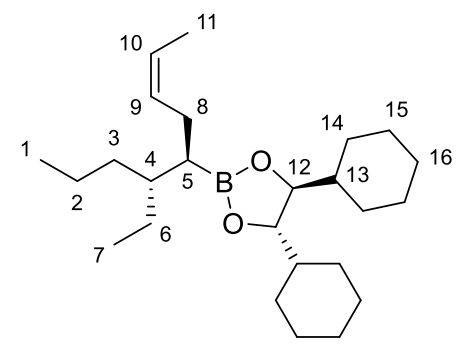

major isomer (E isomer):

${ }^{1} \mathbf{H}$ NMR $\left(500 \mathrm{MHz}, \mathrm{CDCl}_{3}\right): \delta=0.80-0.90(\mathrm{~m}, 6 \mathrm{H}, 1-\mathrm{H}, 7-\mathrm{H}), 0.92-1.05(\mathrm{~m}, 4 \mathrm{H}, 14-\mathrm{H}$, 15-H), 1.09-1.45 (m, 16 H, 2-H, 3-H, 4-H, 5-H, 6-H, 13-H, 14-H', 15-H'), 1.54-1.62 (m, 5 H, 11-H, 14-H' '), 1.67 (m, 2 H, 16-H), 1.71-1.83 (m, 6 H, 14-H' ', 15-H', 16-H'), 2.09 (m, 2 H, 8-H), 3.78 (m, 2 H, 12-H), 5.33-5.46 (m, 2 H, 9-H, 10-H).

${ }^{13} \mathrm{C}$ NMR (125 MHz, $\mathrm{CDCl}_{3}$ ): $\delta=12.3$ (q, C-7), 12.8 (q, C-11), 14.5 (q, C-1), 20.2 (t, C-2), 25.4 (t, C-6), 25.6 (t, C-8), 25.9 (t, C-15), 26.0 (t, C-14), 26.5 (t, C-16), 27.7 (t, C-15)), 28.5 (t, C-14'), 34.6 (t, C-3), 40.6 (d, C-4), 43.1 (d, C-13), 83.2 (d, C-12), 123.4 (d, C-10), 131.3 (d, C-9).

The signal of C-5 could not be detected-

minor isomer ( $Z$ isomer, selected signals):

${ }^{13} \mathrm{C} \mathrm{NMR}\left(125 \mathrm{MHz}, \mathrm{CDCl}_{3}\right.$ ): $\delta=12.2$ (q, C-7), 18.0 (q, C-11), 20.0 (t, C-2), 25.3 (t, C-6), 25.9 (t, C-15), 27.8 (t, C-15'), 28.5 (t, C-14'), 31.4 (t, C-8), 34.4 (t, C-3), 40.5 (d, C-4), 43.1 (d, C-13), 124.7 (d, C-10), 131.9 (d, C-9).

HRMS $(\mathrm{CI}) \mathrm{m} / \mathrm{z}$ calcd for $\mathrm{C}_{25} \mathrm{H}_{46} \mathrm{O}_{2} \mathrm{~B}[\mathrm{M}+\mathrm{H}]^{+}: 389.3585$, found: 389.3585 .

\section{(4S,5S)-4,5-Dicyclohexyl-2-[(5R,6R,E)-6-ethylnon-2-en-5-yl]-1,3,2-dioxaborolane (16d)}

According to GP $4108 \mathrm{mg}(293 \mu \mathrm{mol}) \alpha$-chloroboronic ester 13 were reacted with $1.43 \mathrm{ml}$ $(586 \mu \mathrm{mol})$ allylzinc reagent 9d $(0.41 \mathrm{M}$ in $\mathrm{THF})$. After stirring for $18 \mathrm{~h}$, the reaction was worked up and the crude product was purified by flash chromatography (petroleum ether, ethyl acetate 98:2) to give 16d ( $E: Z=57: 43$ according to GC-FID after oxidation to alcohol 19d) in $75 \%$ yield $\left(98 \mathrm{mg}, 220 \mu \mathrm{mol}\right.$ ) as a colorless oil, $R_{\mathrm{f}}=0.38$ (petroleum ether, ethyl acetate 98:2).

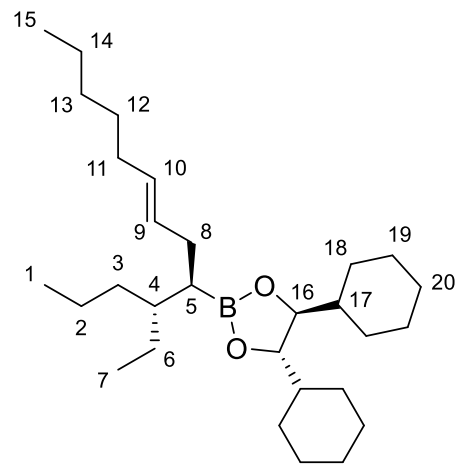


major isomer (E isomer):

${ }^{1} \mathbf{H}$ NMR $\left(500 \mathrm{MHz}, \mathrm{CDCl}_{3}\right): \delta=0.81-0.92(\mathrm{~m}, 9 \mathrm{H}, 1-\mathrm{H}, 7-\mathrm{H}, 15-\mathrm{H}), 0.93-1.05(\mathrm{~m}, 4 \mathrm{H}, 18-\mathrm{H}$, 19-H), 1.09-1.44 (m, 22 H, 2-H, 3-H, 4-H, 5-H, 6-H, 12-H, 13-H, 14-H, 17-H, 18-H', 19-H'), 1.59 (m, 2 H, 18-H' '), 1.67 (m, 2 H, 20-H), 1.71-1.83 (m, 6 H, 18-H' ', 19-H', 20-H'), 1.90 2.16 (m, 4 H, 8-H, 11-H), 3.78 (m, 2 H, 16-H), 5.27-5.43 (m, 2 H, 9-H, 10-H).

${ }^{13} \mathrm{C}$ NMR (125 MHz, $\left.\mathrm{CDCl}_{3}\right): \delta=12.2$ (q, C-7), 14.1 (q, C-15), 14.5 (q, C-1), 20.1 (t, C-2), 22.6 (t, C-14), 25.3 (t, C-6), 25.9 (t, C-19), 26.0 (t, C-18), 26.5 (t, C-20), 27.8 (t, C-19'), 28.5 (t, C-18'), 29.3 (t, C-12), 31.5 (t, C-13), 31.6 (t, C-11), 32.6 (t, C-8), 34.5 (t, C-3), 40.4 (d, C-4), 43.1 (d, C-17), 83.2 (d, C-16), 130.5 (d, C-9), 130.5 (d, C-10).

The signal of $\mathrm{C}-5$ could not be detected-

minor isomer ( $Z$ isomer, selected signals):

${ }^{13} \mathrm{C}$ NMR (125 MHz, $\mathrm{CDCl}_{3}$ ): $\delta=12.3$ (q, C-7), 20.1 (t, C-2), 22.6 (t, C-14), $25.4(\mathrm{t}, \mathrm{C}-6), 25.9$ (t, C-8), 27.2 (t, C-11), 27.8 (t, C-19'), 29.5 (t, C-12), 31.4 (t, C-13), 34.6 (t, C-3), 40.6 (d, C-4), 43.1 (d, C17), 129.8 (d, C-10), 130.3 (d, C-9).

HRMS (CI) m/z calcd for $\mathrm{C}_{29} \mathrm{H}_{53} \mathrm{O}_{2} \mathrm{~B}[\mathrm{M}]^{+}$: 444.4133, found: 444.4139 .

\section{$(4 S, 5 S)-4,5$-Dicyclohexyl-2-[(4R,5R,E)-4-ethyl-9-methyldec-7-en-5-yl]-1,3,2-dioxaboro- lane (16e)}

According to GP $4108 \mathrm{mg}(293 \mu \mathrm{mol}) \alpha$-chloroboronic ester 13 were reacted with $2.25 \mathrm{ml}$ $(586 \mu \mathrm{mol})$ allylzinc reagent $9 \mathrm{e}(0.26 \mathrm{M}$ in THF). After stirring for $18 \mathrm{~h}$, the reaction was worked up and the crude product was purified by flash chromatography (petroleum ether, ethyl acetate $98: 2)$ to give $16 \mathbf{e}\left(E: Z=78: 22\right.$ according to $\left.{ }^{1} \mathrm{H} N M R\right)$ in $68 \%$ yield $(83 \mathrm{mg}, 199 \mu \mathrm{mol})$ as a colorless oil, $\mathrm{R}_{\mathrm{f}}=0.37$ (petroleum ether, ethyl acetate 98:2).

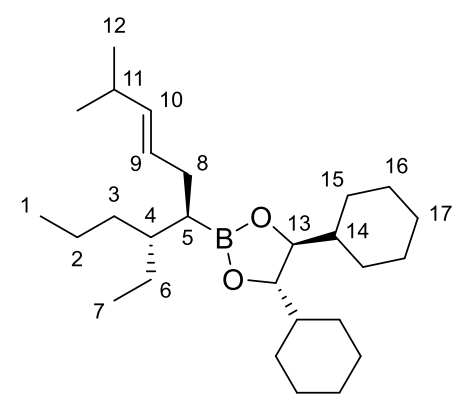

major isomer (E isomer):

${ }^{1} \mathbf{H}$ NMR $\left(500 \mathrm{MHz}, \mathrm{CDCl}_{3}\right): \delta=0.80-0.90(\mathrm{~m}, 6 \mathrm{H}, 1-\mathrm{H}, 7-\mathrm{H}), 0.90-0.98\left(\mathrm{~m},{ }^{3} J_{12,11}=6.6 \mathrm{~Hz}\right.$, $\left.{ }^{3} J_{12}, 11=6.9 \mathrm{~Hz}, 8 \mathrm{H}, 12-\mathrm{H}, 12-\mathrm{H}^{\prime}, 16-\mathrm{H}\right), 1.02(\mathrm{~m}, 2 \mathrm{H}, 15-\mathrm{H}), 1.11-1.44(\mathrm{~m}, 16 \mathrm{H}, 2-\mathrm{H}, 3-\mathrm{H}$, 4-H, 5-H, 6-H, 14-H, 15-H', 16-H'), 1.59 (m, 2 H, 11-H, 15-H'), 1.67 (m, 2 H, 17-H), 1.71$1.83\left(\mathrm{~m}, 6 \mathrm{H}, 15-\mathrm{H}^{\prime},{ }^{\prime}, 16-\mathrm{H}^{\prime},, 17-\mathrm{H}^{\prime}\right), 2.02\left(\mathrm{ddd},{ }^{2} J_{8 \mathrm{a}, 8 \mathrm{~b}}=12.9 \mathrm{~Hz},{ }^{3} J_{8 \mathrm{a}, 5} \approx{ }^{3} J_{8 \mathrm{a}, 9}=6.0 \mathrm{~Hz}, 1 \mathrm{H}\right.$, $\left.8-\mathrm{H}_{\mathrm{a}}\right), 2.10\left(\mathrm{~m}, 1 \mathrm{H}, 8-\mathrm{H}_{\mathrm{b}}\right), 2.20\left(\mathrm{qqd},{ }^{3} J_{11,12} \approx{ }^{3} J_{11,12^{\circ}}=6.6 \mathrm{~Hz},{ }^{3} J_{11,10}=5.4 \mathrm{~Hz}, 1 \mathrm{H}, 11-\mathrm{H}\right)$, $3.78(\mathrm{~m}, 2 \mathrm{H}, 13-\mathrm{H}), 5.34\left(\mathrm{dt},{ }^{3} J_{9,10}=15.4 \mathrm{~Hz},{ }^{3} J_{9,8}=6.0 \mathrm{~Hz}, 1 \mathrm{H}, 9-\mathrm{H}\right), 5.38\left(\mathrm{dd},{ }^{3} J_{10,9}=\right.$ $\left.15.1 \mathrm{~Hz},{ }^{3} J_{10,11}=5.0 \mathrm{~Hz}, 1 \mathrm{H}, 10-\mathrm{H}\right)$.

${ }^{13} \mathrm{C}$ NMR (125 MHz, $\left.\mathrm{CDCl}_{3}\right): \delta=12.2$ (q, C-7), 14.5 (q, C-1), 20.1 (t, C-2), 22.5 (q, C-12), 22.6 (q, C-12'), 25.3 (t, C-6), 25.9 (t, C-16), 26.0 (t, C-15), 26.5 (t, C-17), 27.8 (t, C-16'), 28.5 (t, C-15'), 30.9 (d, C-11), 31.3 (t, C-8), 34.5 (t, C-3), 40.4 (d, C-4), 43.1 (d, C-14), 83.2 (d, C-13), 127.5 (d, C-9), 137.5 (d, C-10).

The signal of $\mathrm{C}-5$ could not be detected. 
minor isomer ( $Z$ isomer, selected signals):

${ }^{1} \mathbf{H}$ NMR $\left(500 \mathrm{MHz}, \mathrm{CDCl}_{3}\right): \delta=2.63\left(\mathrm{dqq},{ }^{3} J_{11,10}=9.1 \mathrm{~Hz},{ }^{3} J_{11,12} \approx{ }^{3} J_{11,12^{*}}=6.5 \mathrm{~Hz}, 1 \mathrm{H}\right.$, $11-\mathrm{H}), 5.13\left(\mathrm{dd},{ }^{3} J_{10,9}=10.7 \mathrm{~Hz},{ }^{3} J_{10,11}=9.1 \mathrm{~Hz}, 1 \mathrm{H}, 10-\mathrm{H}\right), 5.21\left(\mathrm{dt},{ }^{3} J_{9,10}=10.7 \mathrm{~Hz},{ }^{3} J_{9,8}=\right.$ $7.3 \mathrm{~Hz}, 1 \mathrm{H}, 9-\mathrm{H})$.

${ }^{13} \mathrm{C}$ NMR (125 MHz, $\mathrm{CDCl}_{3}$ ): $\delta=20.2$ (t, C-2), 23.2 (q, C-12), 23.3 (q, C-12'), 25.4 (t, C-6), 26.5 (d, C-11), 34.6 (t, C-3), 40.5 (d, C-4), 43.1 (d, C-14), 127.9 (d, C-9), 137.2 (d, C-10).

HRMS (CI) m/z calcd for $\mathrm{C}_{27} \mathrm{H}_{50} \mathrm{O}_{2} \mathrm{~B}[\mathrm{M}+\mathrm{H}]^{+}:$417.3898, found: 417.3886.

\section{(S,Z)-Hex-4-en-2-ol (17c)}

$73 \mathrm{mg}(229 \mu \mathrm{mol})$ boronic ester $\mathbf{1 4 c}, 106 \mu \mathrm{l}(33 \%$ in water, $\rho=1.11 \mathrm{~g} / \mathrm{ml}, 1.15 \mathrm{mmol})$ hydrogen peroxide and $46 \mathrm{mg}$ ( $1.15 \mathrm{mmol})$ sodium hydroxide were reacted according to GP 2 . After $2.5 \mathrm{~h}$, the reaction was worked up and the crude product was purified by flash chromatography (pentane, diethyl ether 7:3) to give 17c $\left(E: Z=24: 76\right.$ according to ${ }^{1} \mathrm{H}$ NMR; $75 \mathrm{wt}-\%$ in pentane, diethyl ether) in $46 \%$ yield $\left(14.1 \mathrm{mg}, 106 \mu \mathrm{mol}\right.$ ) as a colorless oil, $\mathrm{R}_{\mathrm{f}}=0.20$ (pentane, diethyl ether 6:4). NMR spectra of $\mathbf{1 7} \mathbf{c}$ were in accordance with previously published data. ${ }^{15}$

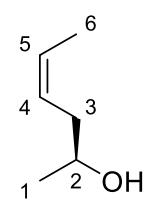

major isomer ( $\mathrm{Z}$ isomer):

${ }^{1} \mathbf{H}$ NMR $\left(500 \mathrm{MHz}, \mathrm{CDCl}_{3}\right): \delta=1.21\left(\mathrm{~d},{ }^{3} J_{1,2}=6.0 \mathrm{~Hz}, 3 \mathrm{H}, 1-\mathrm{H}\right), 1.59(\mathrm{bs}, 1 \mathrm{H}, \mathrm{OH}), 1.64$ $(\mathrm{m}, 3 \mathrm{H}, 6-\mathrm{H}), 2.18\left(\mathrm{~m}, 1 \mathrm{H}, 3-\mathrm{H}_{\mathrm{a}}\right), 2.26\left(\mathrm{~m}, 1 \mathrm{H}, 3-\mathrm{H}_{\mathrm{b}}\right), 3.81(\mathrm{~m}, 1 \mathrm{H}, 2-\mathrm{H}), 5.43(\mathrm{~m}, 1 \mathrm{H}, 4-\mathrm{H})$, $5.65\left(\mathrm{dqt},{ }^{3} J_{5,4}=11.0 \mathrm{~Hz},{ }^{3} J_{5,6}=6.9 \mathrm{~Hz},{ }^{4} J_{5,3}=1.6 \mathrm{~Hz}, 1 \mathrm{H}, 5-\mathrm{H}\right)$.

${ }^{13}$ C NMR (125 MHz, $\mathrm{CDCl}_{3}$ ): $\delta=13.0$ (q, C-6), 22.8 (q, C-1), 36.7 (t, C-3), 67.7 (d, C-2), 126.0 (d, C-5), 127.3 (d, C-4).

minor isomer (E isomer, selected signals):

${ }^{1} \mathbf{H}$ NMR $\left(500 \mathrm{MHz}, \mathrm{CDCl}_{3}\right): \delta=1.18\left(\mathrm{~d},{ }^{3} J_{1,2}=6.0 \mathrm{~Hz}, 3 \mathrm{H}, 1-\mathrm{H}\right), 1.69$ (m, $\left.3 \mathrm{H}, 6-\mathrm{H}\right), 2.07$ $\left(\mathrm{m}, 1 \mathrm{H}, 3-\mathrm{H}_{\mathrm{a}}\right), 5.56\left(\mathrm{dqt},{ }^{3} J_{5,4}=15.1 \mathrm{~Hz},{ }^{3} J_{5,6}=6.3 \mathrm{~Hz},{ }^{4} J_{5,3}=1.3 \mathrm{~Hz}, 1 \mathrm{H}, 5-\mathrm{H}\right)$.

${ }^{13} \mathrm{C}$ NMR (125 MHz, $\mathrm{CDCl}_{3}$ ): $\delta=18.1$ (q, C-6), 22.6 (q, C-1), 67.2 (d, C-2), 127.1 (d, C-5), 129.0 (d, C-4).

\section{(S,Z)-Dec-4-en-2-ol (17d)}

$95 \mathrm{mg}(254 \mu \mathrm{mol})$ boronic ester 14d, $118 \mu \mathrm{l}(33 \%$ in water, $\rho=1.11 \mathrm{~g} / \mathrm{ml}, 1.27 \mathrm{mmol})$ hydrogen peroxide and $51 \mathrm{mg}(1.27 \mathrm{mmol})$ sodium hydroxide were reacted according to GP 2 . After $2.5 \mathrm{~h}$, the reaction was worked up and the crude product was purified by flash chromatography (pentane, diethyl ether $3: 1)$ to give 17d $\left(E: Z=37: 63\right.$ according to $\left.{ }^{1} \mathrm{H} N M R\right)$ in $86 \%$ yield ( $34 \mathrm{mg}, 218 \mu \mathrm{mol}$ ) as a colorless oil, $\mathrm{R}_{\mathrm{f}}=0.14$ (pentane, diethyl ether 8:2). NMR spectra of $\mathbf{1 7 d}$ were in accordance with previously published data. ${ }^{16}$

\footnotetext{
${ }^{15}$ Adam, W.; Saha-Möller, C. R.; Schmid, K. S., Synthesis of 4,6-Dideoxyfuranoses through the Regioselective and Diastereoselective Oxyfunctionalization of a Dimethylphenylsilyl-Substituted Chiral Homoallylic Alcohol. J. Org. Chem. 2001, 66, 7365-7371.

${ }^{16}$ Maryanoff, B. E.; Reitz, A. B.; Duhl-Emswiler, B. A., Stereochemistry of the Wittig reaction. Effect of nucleophilic groups in the phosphonium ylide. J. Am. Chem. Soc. 1985, 107, 217-226.
} 


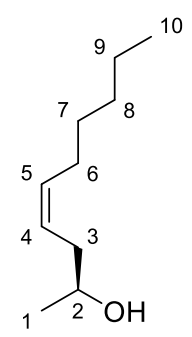

major isomer ( $Z$ isomer):

${ }^{1} \mathbf{H}$ NMR $\left(500 \mathrm{MHz}, \mathrm{CDCl}_{3}\right): \delta=0.88\left(\mathrm{t},{ }^{3} J_{10,9}=7.3 \mathrm{~Hz}, 3 \mathrm{H}, 10-\mathrm{H}\right), 1.21\left(\mathrm{~d},{ }^{3} J_{1,2}=6.3 \mathrm{~Hz}\right.$, $3 \mathrm{H}, 1-\mathrm{H}, 1.24-1.40$ (m, $6 \mathrm{H}, 7-\mathrm{H}, 8-\mathrm{H}, 9-\mathrm{H}), 1.62$ (bs, $1 \mathrm{H}, \mathrm{OH}), 2.05$ (m, $2 \mathrm{H}, 6-\mathrm{H}), 2.20$ (m, $2 \mathrm{H}, 3-\mathrm{H}), 3.80$ (m, $1 \mathrm{H}, 2-\mathrm{H}), 5.39$ (m, $1 \mathrm{H}, 4-\mathrm{H}), 5.55$ (m, $1 \mathrm{H}, 5-\mathrm{H})$.

${ }^{13}$ C NMR (125 MHz, CDCl 3$): \delta=14.0$ (q, C-10), 22.5 (t, C-9), 22.7 (q, C-1), 27.4 (t, C-6), 29.3 (t, C-7), 31.5 (t, C-8), 37.1 (t, C-3), 67.7 (d, C-2), 125.0 (d, C-5), 133.6 (d, C-4).

minor isomer (E isomer, selected signals):

${ }^{1} \mathbf{H}$ NMR $\left(500 \mathrm{MHz}, \mathrm{CDCl}_{3}\right): \delta=1.18\left(\mathrm{~d},{ }^{3} J_{1,2}=6.3 \mathrm{~Hz}, 3 \mathrm{H}, 1-\mathrm{H}\right), 1.56(\mathrm{bs}, 1 \mathrm{H}, \mathrm{OH})$.

${ }^{13} \mathrm{C}$ NMR $\left(125 \mathrm{MHz}, \mathrm{CDCl}_{3}\right.$ ): $\delta=22.5$ (t, C-9), 22.6 (q, C-1), 29.1 (t, C-7), 31.4 (t, C-8), 32.6 (t, C-6), 42.5 (t, C-3), 67.1 (d, C-2), 125.7 (d, C-5), 134.9 (d, C-4).

HRMS (CI) m/z calcd for $\mathrm{C}_{10} \mathrm{H}_{21} \mathrm{O}[\mathrm{M}+\mathrm{H}]^{+}:$157.1587, found: 157.1558 .

\section{(R,Z)-1-Cyclohexylpent-3-en-1-ol (18c)}

$107 \mathrm{mg}(277 \mu \mathrm{mol})$ boronic ester 15c, $129 \mu \mathrm{l}(33 \%$ in water, $\rho=1.11 \mathrm{~g} / \mathrm{ml}, 1.38 \mathrm{mmol})$ hydrogen peroxide and $55 \mathrm{mg}(1.38 \mathrm{mmol})$ sodium hydroxide were reacted according to GP 2 . After $1 \mathrm{~h}$, the reaction was worked up and the crude product was purified by flash chromatography (pentane, diethyl ether 8:2) to give 18c $\left(E: Z=39: 61\right.$ according to ${ }^{1} \mathrm{H}$ NMR) in $90 \%$ yield $\left(42 \mathrm{mg}, 250 \mu \mathrm{mol}\right.$ ) as a colorless oil, $\mathrm{R}_{\mathrm{f}}=0.42$ (pentane, diethyl ether 6:4). NMR spectra of $18 \mathbf{c}$ were in accordance with previously published data. ${ }^{17}$

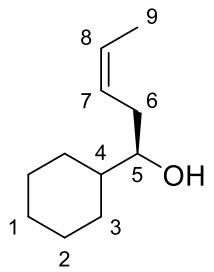

major isomer ( $\mathrm{Z}$ isomer):

${ }^{1} \mathbf{H}$ NMR (500 MHz, $\left.\mathrm{CDCl}_{3}\right): \delta=0.98-1.30$ (m, $\left.5 \mathrm{H}, 1-\mathrm{H}, 2-\mathrm{H}, 3-\mathrm{H}\right), 1.35$ (m, $\left.1 \mathrm{H}, 4-\mathrm{H}\right), 1.52$ $\left(\mathrm{d},{ }^{3} J_{\mathrm{OH}, 5}=3.2 \mathrm{~Hz}, 1 \mathrm{H}, \mathrm{OH}\right), 1.62-1.71\left(\mathrm{~m}, 5 \mathrm{H}, 1-\mathrm{H}^{\prime}, 3-\mathrm{H}^{\prime}, 9-\mathrm{H}\right), 1.75$ (m, $\left.2 \mathrm{H}, 2-\mathrm{H}^{\prime}\right), 1.86$ (m, $1 \mathrm{H}, 3-\mathrm{H}$ ') $2.25(\mathrm{~m}, 2 \mathrm{H}, 6-\mathrm{H}), 3.38(\mathrm{~m}, 1 \mathrm{H}, 5-\mathrm{H}), 5.44(\mathrm{~m}, 1 \mathrm{H}, 7-\mathrm{H}), 5.66\left(\mathrm{dqt},{ }^{3} J_{8,7}=\right.$ $\left.10.7 \mathrm{~Hz},{ }^{3} J_{8,9}=6.9 \mathrm{~Hz},{ }^{4} J_{8,6}=1.6 \mathrm{~Hz}, 1 \mathrm{H}, 8-\mathrm{H}\right)$.

${ }^{13}$ C NMR (125 MHz, CDCl 3 ): $\delta=13.0$ (q, C-9), 26.1 (t, C-2), 26.3 (t, C-2'), 26.5 (t, C-1), 28.1 (t, C-3), 29.2 (t, C-3'), 31.8 (t, C-6), 43.1 (d, C-4), 75.5 (d, C-5), 126.7 (d, C-7), 127.3 (d, C-8).

\footnotetext{
${ }^{17}$ Incerti-Pradillos, C. A.; Kabeshov, M. A.; Malkov, A. M., Highly Stereoselective Synthesis of Z-Homoallylic Alcohols by Kinetic Resolution of Racemic Secondary Allyl Boronates. Angew. Chem. Int. Ed. 2013, 52, 53385341 .
} 
minor isomer (E isomer, selected signals):

${ }^{1} \mathbf{H}$ NMR $\left(500 \mathrm{MHz}, \mathrm{CDCl}_{3}\right): \delta=1.56\left(\mathrm{~d},{ }^{3} \mathrm{~J}_{\mathrm{OH}, 5}=3.5 \mathrm{~Hz}, 1 \mathrm{H}, \mathrm{OH}\right), 2.04\left(\mathrm{~m}, 1 \mathrm{H}, 6-\mathrm{H}_{\mathrm{a}}\right), 2.25$ $\left(\mathrm{m}, 1 \mathrm{H}, 6-\mathrm{H}_{\mathrm{b}}\right), 3.32$ (dddd, ${ }^{3} J_{5,6 \mathrm{a}}=8.6 \mathrm{~Hz},{ }^{3} J_{5,4}=5.7 \mathrm{~Hz},{ }^{3} J_{5,6 \mathrm{~b}} \approx{ }^{3} J_{5, \mathrm{OH}}=3.0 \mathrm{~Hz}, 1 \mathrm{H}, 5-\mathrm{H}$ ), 5.56 (dqt, $\left.{ }^{3} J_{8,7}=15.1 \mathrm{~Hz},{ }^{3} J_{8,9}=6.3 \mathrm{~Hz},{ }^{4} J_{8,6}=1.3 \mathrm{~Hz}, 1 \mathrm{H}, 8-\mathrm{H}\right)$.

${ }^{13}$ C NMR (125 MHz, CDCl 3 ): $\delta=18.0$ (q, C-9), 26.3 (t, C-2'), 28.2 (t, C-3), 29.1 (t, C-3'), 37.5 (t, C-6), 43.0 (d, C-4), 74.9 (d, C-5), 127.6 (d, C-7), 128.9 (d, C-8).

\section{(R,E)-1-Cyclohexylnon-3-en-1-ol (18d)}

$95 \mathrm{mg}(215 \mu \mathrm{mol})$ boronic ester $\mathbf{1 5 d}, 100 \mu \mathrm{l}(33 \%$ in water, $\rho=1.11 \mathrm{~g} / \mathrm{ml}, 1.07 \mathrm{mmol})$ hydrogen peroxide and $43 \mathrm{mg}(1.07 \mathrm{mmol})$ sodium hydroxide were reacted according to GP 2 . After $2.5 \mathrm{~h}$, the reaction was worked up and the crude product was purified by flash chromatography (pentane, diethyl ether 8:2) to give 18d $(E: Z=59: 41$ according to GC-FID) in $87 \%$ yield (42 mg, $187 \mu \mathrm{mol})$ as a colorless oil, $\mathrm{R}_{\mathrm{f}}=0.31$ (pentane, diethyl ether 8:2).

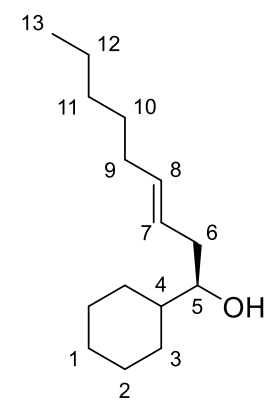

major isomer (E isomer):

${ }^{1} \mathbf{H}$ NMR $\left(500 \mathrm{MHz}, \mathrm{CDCl}_{3}\right): \delta=0.87\left(\mathrm{t},{ }^{3} J_{13,12}=7.1 \mathrm{~Hz}, 3 \mathrm{H}, 13-\mathrm{H}\right), 0.95-1.40(\mathrm{~m}, 12 \mathrm{H}, 1-\mathrm{H}$, 2-H, 3-H, 4-H, 10-H, 11-H, 12-H), $1.62\left(\mathrm{~d},{ }^{3} \mathrm{~J}_{\mathrm{OH}, 5}=3.2 \mathrm{~Hz}, 1 \mathrm{H}, \mathrm{OH}\right), 1.63-1.70\left(\mathrm{~m}, 2 \mathrm{H}, 1-\mathrm{H}^{\prime}\right.$, 3-H'), 1.75 (m, 2 H, 2-H'), 1.85 (m, 1 H, 3-H'), 1.97-2.30 (m, 4 H, 6-H, 9-H), 3.31 (dddd, $\left.{ }^{3} J_{5,6 \mathrm{a}}=8.4 \mathrm{~Hz},{ }^{3} J_{5,4}=5.8 \mathrm{~Hz},{ }^{3} J_{5,6 \mathrm{~b}} \approx{ }^{3} J_{5, \mathrm{OH}}=3.2 \mathrm{~Hz}, 1 \mathrm{H}, 5-\mathrm{H}\right), 5.39(\mathrm{~m}, 1 \mathrm{H}, 7-\mathrm{H}), 5.56(\mathrm{~m}$, $1 \mathrm{H}, 8-\mathrm{H})$.

${ }^{13} \mathrm{C}$ NMR (125 MHz, $\left.\mathrm{CDCl}_{3}\right): \delta=14.0$ (q, C-13), 22.5 (t, C-12), 26.1 (t, C-2), 26.3 (t, C-2'), 26.5 (t, C-1), 28.2 (t, C-3), 29.1 (t, C-3'), 29.1 (t, C-10), 31.4 (t, C-11), 32.6 (t, C-9), 37.5 (t, C-6), 42.9 (d, C-4), 74.9 (d, C-5), 126.2 (d, C-7), 133.7 (d, C-8).

minor isomer ( $Z$ isomer, selected signals):

${ }^{1} \mathbf{H}$ NMR $\left(500 \mathrm{MHz}, \mathrm{CDCl}_{3}\right): \delta=1.57\left(\mathrm{~d},{ }^{3} \mathrm{~J}_{\mathrm{OH}, 5}=3.5 \mathrm{~Hz}, 1 \mathrm{H}, \mathrm{OH}\right), 3.35$ (m, $\left.1 \mathrm{H}, 5-\mathrm{H}\right)$.

${ }^{13} \mathrm{C} \mathrm{NMR}\left(125 \mathrm{MHz}, \mathrm{CDCl}_{3}\right): \delta=22.5$ (t, C-12), 27.3 (t, C-9), 28.1 (t, C-3), $29.2(\mathrm{t}, \mathrm{C}-3$ '), 29.3 (t, C-10), 31.5 (t, C-11), 32.1 (t, C-6), 43.0 (d, C-4), 75.5 (d, C-5), 125.6 (d, C-7), 134.5 (d, C-8).

GC-FID (CP-Chirasil-Dex $C B, \mathrm{~T}_{0}[5 \mathrm{~min}]=40{ }^{\circ} \mathrm{C}, 5{ }^{\circ} \mathrm{C} / \mathrm{min}$ to $200{ }^{\circ} \mathrm{C}$ [5 min], injector $250{ }^{\circ} \mathrm{C}$, detector $275^{\circ} \mathrm{C}, \mathrm{t}_{\mathrm{R}}(R, Z)-\mathbf{1 8 d}=36.43 \mathrm{~min}, \mathrm{t}_{\mathrm{R}}(R, E)-\mathbf{1 8 d}=36.66 \mathrm{~min}$.

HRMS (CI) m/z calcd for $\mathrm{C}_{15} \mathrm{H}_{29} \mathrm{O}[\mathrm{M}+\mathrm{H}]^{+}:$225.2213, found: 225.2219 .

\section{$(5 R, 6 R, Z)-6-E t h y l n o n-2-e n-5-o l ~(19 c)$}

$68 \mathrm{mg}(175 \mu \mathrm{mol})$ boronic ester 16c, $81 \mu \mathrm{l}(33 \%$ in water, $\rho=1.11 \mathrm{~g} / \mathrm{ml}, 875 \mu \mathrm{mol})$ hydrogen peroxide and $35 \mathrm{mg}(875 \mu \mathrm{mol})$ sodium hydroxide were reacted according to GP 2 . After $1 \mathrm{~h}$, the reaction was worked up and the crude product was purified by flash chromatography (pentane, diethyl ether 8:2) to give 19c $\left(E: Z=37: 63\right.$ according to $\left.{ }^{1} \mathrm{H} N M R\right)$ in $58 \%$ yield $\left(17 \mathrm{mg}, 101 \mu \mathrm{mol}\right.$ ) as a colorless oil, $\mathrm{R}_{\mathrm{f}}=0.25$ (pentane, diethyl ether 8:2). 


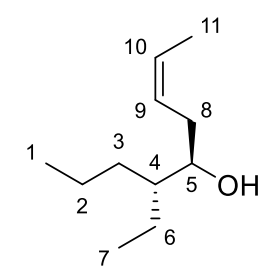

major isomer ( $Z$ isomer):

${ }^{1} \mathbf{H}$ NMR $\left(500 \mathrm{MHz}, \mathrm{CDCl}_{3}\right): \delta=0.87-0.94(\mathrm{~m}, 6 \mathrm{H}, 1-\mathrm{H}, 7-\mathrm{H}), 1.16-1.53(\mathrm{~m}, 8 \mathrm{H}, 2-\mathrm{H}, 3-\mathrm{H}$, 4-H, 6-H, OH), $1.65(\mathrm{~m}, 3 \mathrm{H}, 11-\mathrm{H}), 2.21(\mathrm{~m}, 2 \mathrm{H}, 8-\mathrm{H}), 3.62\left(\mathrm{dddd},{ }^{3} J_{5,6 \mathrm{a}}=8.4 \mathrm{~Hz},{ }^{3} J_{5,6 \mathrm{~b}} \approx\right.$ $\left.{ }^{3} J_{5,4} \approx^{3} J_{5, \mathrm{OH}}=4.1 \mathrm{~Hz}, 1 \mathrm{H}, 5-\mathrm{H}\right), 5.44(\mathrm{~m}, 1 \mathrm{H}, 9-\mathrm{H}), 5.65\left(\mathrm{dqt},{ }^{3} J_{10,9}=10.7 \mathrm{~Hz},{ }^{3} J_{10,11}=6.9 \mathrm{~Hz}\right.$, $\left.{ }^{4} J_{10,8}=1.3 \mathrm{~Hz}, 1 \mathrm{H}, 10-\mathrm{H}\right)$.

${ }^{13} \mathrm{C}$ NMR (125 MHz, $\mathrm{CDCl}_{3}$ ): $\delta=11.7$ (q, C-7), 13.0 (q, C-11), 14.5 (q, C-1), 20.5 (t, C-6), 21.8 (t, C-2), 31.7 (t, C-8), 31.9 (t, C-3), 44.3 (d, C-4), 72.9 (d, C-5), 127.0 (d, C-9), 127.1 (d, $\mathrm{C}-10)$.

minor isomer ( $E$ isomer, selected signals):

${ }^{1} \mathbf{H}$ NMR $\left(500 \mathrm{MHz}, \mathrm{CDCl}_{3}\right): \delta=1.69(\mathrm{~m}, 3 \mathrm{H}, 11-\mathrm{H}), 2.05\left(\mathrm{ddd},{ }^{2} J_{8 \mathrm{a}, 8 \mathrm{~b}}=13.9 \mathrm{~Hz},{ }^{3} J_{8 \mathrm{a}, 5} \approx\right.$ $\left.{ }^{3} J_{8 \mathrm{a}, 9}=8.7 \mathrm{~Hz}, 1 \mathrm{H}, 8-\mathrm{H}_{\mathrm{a}}\right), 2.21\left(\mathrm{~m}, 1 \mathrm{H}, 8-\mathrm{H}_{\mathrm{b}}\right), 3.57(\mathrm{~m}, 1 \mathrm{H}, 5-\mathrm{H}), 5.56\left(\mathrm{dqt},{ }^{3} J_{10,9}=15.1 \mathrm{~Hz}\right.$, $\left.{ }^{3} J_{10,11}=6.3 \mathrm{~Hz},{ }^{4} J_{10,8}=0.9 \mathrm{~Hz}, 1 \mathrm{H}, 10-\mathrm{H}\right)$.

${ }^{13}$ C NMR (125 MHz, CDCl 3 ): $\delta=11.7$ (q, C-7), 18.0 (q, C-11), 20.5 (t, C-6), 21.8 (t, C-2), 31.8 (t, C-3), 37.4 (t, C-8), 42.2 (d, C-4), 72.4 (d, C-5), 128.0 (d, C-9), 128.7 (d, C-10).

HRMS (CI) m/z calcd for $\mathrm{C}_{11} \mathrm{H}_{22} \mathrm{O}[\mathrm{M}]^{+}:$170.1665, found: 170.1679 .

\section{$(4 R, 5 R, E)-4-E t h y l t r i d e c-7-e n-5-o l ~(19 d)$}

$85 \mathrm{mg}(191 \mu \mathrm{mol})$ boronic ester 16d, $89 \mu \mathrm{l}(33 \%$ in water, $\rho=1.11 \mathrm{~g} / \mathrm{ml}, 956 \mu \mathrm{mol})$ hydrogen peroxide and $38 \mathrm{mg}(956 \mu \mathrm{mol})$ sodium hydroxide were reacted according to GP 2 . After $1.5 \mathrm{~h}$, the reaction was worked up and the crude product was purified by flash chromatography (pentane, diethyl ether 8:2) to give 19d $(E: Z=57: 43$ according to GC-FID) in $74 \%$ yield (32 $\mathrm{mg}, 141 \mu \mathrm{mol}$ ) as a colorless oil, $\mathrm{R}_{\mathrm{f}}=0.34$ (pentane, diethyl ether 8:2).

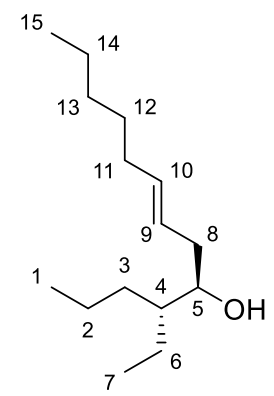

major isomer (E isomer):

${ }^{1} \mathbf{H}$ NMR $\left(500 \mathrm{MHz}, \mathrm{CDCl}_{3}\right): \delta=0.85-0.95(\mathrm{~m}, 9 \mathrm{H}, 1-\mathrm{H}, 7-\mathrm{H}, 15-\mathrm{H}), 1.17-1.51(\mathrm{~m}, 13 \mathrm{H}$, 2-H, 3-H, 4-H, 5-H, 12-H, 13-H, 14-H), 1.54 (d, $\left.{ }^{3} J_{\mathrm{OH}, 5}=2.8 \mathrm{~Hz}, 1 \mathrm{H}, \mathrm{OH}\right), 1.98-2.09(\mathrm{~m}, 3 \mathrm{H}$, 8- $\left.\mathrm{H}_{\mathrm{a}}, 11-\mathrm{H}\right), 2.21\left(\mathrm{~m}, 1 \mathrm{H}, 8-\mathrm{H}_{\mathrm{b}}\right), 3.56(\mathrm{~m}, 1 \mathrm{H}, 5-\mathrm{H}), 5.40(\mathrm{~m}, 1 \mathrm{H}, 11-\mathrm{H}), 5.55(\mathrm{~m}, 1 \mathrm{H}, 10-\mathrm{H})$.

${ }^{13} \mathrm{C}$ NMR (125 MHz, $\mathrm{CDCl}_{3}$ ): $\delta=11.6$ (q, C-7), 14.0 (q, C-15), 14.5 (q, C-1), 20.4 (t, C-6), 21.7 (t, C-2), 22.5 (t, C-14), 29.1 (t, C-12), 31.4 (t, C-13), 31.7 (t, C-3), 32.6 (t, C-11), 37.4 (t, C-8), 44.1 (d, C-4), 72.2 (d, C-5), 126.5 (d, C-9), 134.7 (d, C-10). 
minor isomer ( $Z$ isomer, selected signals):

${ }^{1} \mathbf{H}$ NMR $\left(500 \mathrm{MHz}, \mathrm{CDCl}_{3}\right): \delta=1.69$ (bs, $\left.1 \mathrm{H}, \mathrm{OH}\right), 2.03(\mathrm{~m}, 2 \mathrm{H}, 8-\mathrm{H}), 2.21(\mathrm{~m}, 2 \mathrm{H}, 11-\mathrm{H})$, $3.60(\mathrm{~m}, 1 \mathrm{H}, 5-\mathrm{H})$.

${ }^{13}$ C NMR (125 MHz, CDCl 3 ): $\delta=11.7$ (q, C-7), 20.5 (t, C-6), 21.7 (t, C-2), 22.5 (t, C-14), 27.4 (t, C-11), 29.3 (t, C-12), 31.5 (t, C-13), 31.8 (t, C-3), 32.0 (t, C-8), 44.2 (d, C-4), 72.8 (d, C-5), 125.8 (d, C-9), 133.4 (d, C-10).

GC-FID $\left(C P\right.$-Chirasil-Dex $C B, \mathrm{~T}_{0}[5 \mathrm{~min}]=40{ }^{\circ} \mathrm{C}, 5{ }^{\circ} \mathrm{C} / \mathrm{min}$ to $200{ }^{\circ} \mathrm{C}[5 \mathrm{~min}]$, injector $250{ }^{\circ} \mathrm{C}$, detector $275^{\circ} \mathrm{C}, \mathrm{t}_{\mathrm{R}}(R, Z)-\mathbf{1 9 d}=32.97 \mathrm{~min}, \mathrm{t}_{\mathrm{R}}(R, E)-\mathbf{1 9 d}=33.31 \mathrm{~min}$.

HRMS (CI) $\mathrm{m} / \mathrm{z}$ calcd for $\mathrm{C}_{15} \mathrm{H}_{31} \mathrm{O}[\mathrm{M}+\mathrm{H}]^{+}: 227.2369$, found: 227.2362 .

\section{tert-Butyl $\{[(R)-5-C h l o r o-5-([4 S, 5 S]-4,5-$ dicyclohexyl-1,3,2-dioxaborolan-2-yl)pentyl $]$ oxy $\}$ dimethylsilane (20-Cl)}

tert-Butyl $\{4-[(4 S, 5 S)-4,5$-dicyclohexyl-1,3,2-dioxaborolan-2-yl]butoxy $\}$ dimethylsilane was prepared as described previously. ${ }^{14}$

According to GP 1a $499 \mathrm{mg}(1.18 \mathrm{mmol}) \mathbf{2 0}, 228 \mu \mathrm{l}(\rho=1.32 \mathrm{~g} / \mathrm{ml}, 3.54 \mathrm{mmol})$ anhydrous DCM, $923 \mu \mathrm{l}(1.6 \mathrm{M}$ in hexanes, $1.48 \mathrm{mmol}) n$-butyllithium, $227 \mu \mathrm{l}(\rho=0.71 \mathrm{~g} / \mathrm{ml}, 1.59 \mathrm{mmol})$ diisopropylamine and $483 \mathrm{mg}(3.54 \mathrm{mmol})$ zinc chloride were reacted. After stirring at room temperature for $2 \mathrm{~h}$, the reaction was worked up to obtain crude $\mathbf{2 0}-\mathbf{C l}$ in $98 \%$ yield $(547 \mathrm{mg}$, $1.16 \mathrm{mmol})$ as a pale yellow oil, $\alpha_{D}^{20}=-43.7\left(\mathrm{c}=1.0, \mathrm{CHCl}_{3}\right) . \mathbf{2 0}-\mathbf{C l}$ was used in the next step without further purification.

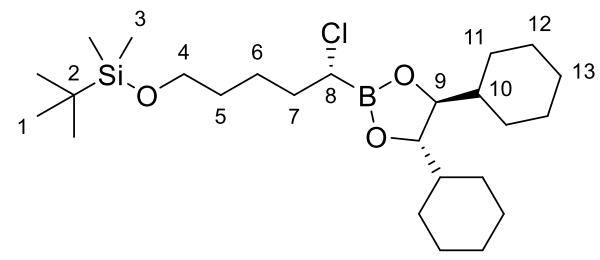

${ }^{1} \mathbf{H}$ NMR $\left(400 \mathrm{MHz}, \mathrm{CDCl}_{3}\right): \delta=0.04(\mathrm{~s}, 6 \mathrm{H}, 3-\mathrm{H}), 0.89(\mathrm{~s}, 9 \mathrm{H}, 1-\mathrm{H}), 0.98(\mathrm{~m}, 2 \mathrm{H}, 12-\mathrm{H})$, 1.07 (m, 2 H, 11-H), 1.11-1.30 (m, 6 H, 11-H', 12-H'), 1.37 (m, 2 H, 10-H), 1.42-1.64 (m, $6 \mathrm{H}$, 5-H, 6-H, 11-H' '), 1.68 (m, 2 H, 13-H), 1.72-1.81 (m, $6 \mathrm{H}, 11-\mathrm{H}^{\prime}, ', 12-\mathrm{H}^{\prime}, 11-\mathrm{H}$ '), 1.85 (m, $2 \mathrm{H}, 7-\mathrm{H}), 3.46\left(\mathrm{dd},{ }^{3} J_{8,7 \mathrm{a}}=8.4 \mathrm{~Hz},{ }^{3} J_{8,7 \mathrm{~b}}=6.4 \mathrm{~Hz}, 1 \mathrm{H}, 8-\mathrm{H}\right), 3.61\left(\mathrm{t},{ }^{3} J_{4,5}=6.2 \mathrm{~Hz}, 2 \mathrm{H}, 4-\mathrm{H}\right)$, $3.95(\mathrm{~m}, 2 \mathrm{H}, 9-\mathrm{H})$.

${ }^{13}$ C NMR (100 MHz, $\left.\mathrm{CDCl}_{3}\right): \delta=-5.3$ (q, C-3), 18.3 (s, C-2), 23.7 (t, C-6), 25.8 (t, C-12), 25.9 (t, C-11), 26.0 (q, C-1), 26.4 (t, C-13), 27.2 (t, C-12'), 28.1 (t, C-11'), 32.3 (t, C-5), 34.0 (t, C-7), 42.8 (d, C-10), 63.0 (t, C-4), 84.1 (d, C-9).

The signal of C-8 could not be detected.

HRMS (CI) m/z calcd for $\mathrm{C}_{25} \mathrm{H}_{49} \mathrm{O}_{3} \mathrm{BClSi}[\mathrm{M}+\mathrm{H}]^{+}$: 471.3227 , found: 471.3244 .

\section{(4S,5S)-4,5-Dicyclohexyl-2-[(S,E)-7,7-dimethyl-1-phenyloct-5-en-3-yl]-1,3,2-dioxaboro- lane (21)}

According to GP $498 \mathrm{mg}(208 \mu \mathrm{mol}) \alpha$-chloroboronic ester $\mathbf{2 0 - C l}$ were reacted with $1.13 \mathrm{ml}$ $(416 \mu \mathrm{mol})$ allylzinc reagent $9 \mathrm{~g}(0.37 \mathrm{M}$ in THF). After stirring for $18 \mathrm{~h}$, the reaction was worked up and the crude product was purified by flash chromatography (petroleum ether, ethyl acetate 98:2) to give 21 ( $E: Z>96: 4$ according to ${ }^{1} \mathrm{H}$ NMR) in $87 \%$ yield $(100 \mathrm{mg}, 199 \mu \mathrm{mol})$ as a colorless oil, $\mathrm{R}_{\mathrm{f}}=0.16$ (petroleum ether, ethyl acetate 98:2); $\alpha_{D}^{20}=-16.2\left(\mathrm{c}=0.5, \mathrm{CHCl}_{3}\right)$. An analytically pure sample of $\mathbf{2 1}$ was obtained by reversed-phase flash chromatography $\left(\mathrm{H}_{2} \mathrm{O}\right.$, $70 \% \mathrm{MeCN} \rightarrow 100 \% \mathrm{MeCN}$ ). 


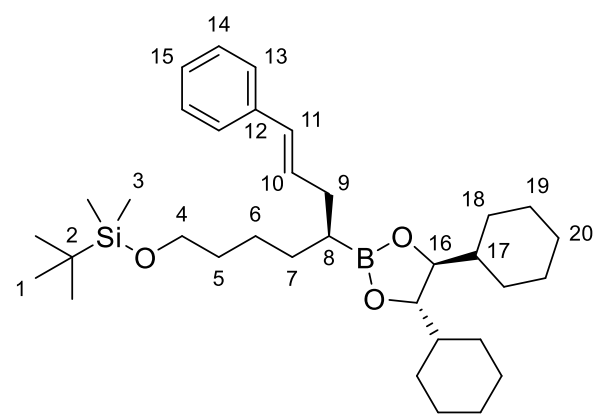

${ }^{1} \mathbf{H}$ NMR $\left(500 \mathrm{MHz}, \mathrm{CDCl}_{3}\right): \delta=0.04(\mathrm{~s}, 6 \mathrm{H}, 3-\mathrm{H}), 0.89$ (s, $\left.9 \mathrm{H}, 1-\mathrm{H}\right), 0.95(\mathrm{~m}, 2 \mathrm{H}, 19-\mathrm{H})$, 1.02 (m, 2 H, 18-H), 1.07-1.23 (m, 7 H, 8-H, 18-H', 19-H'), 1.27 (m, 2 H, 17-H), 1.32-1.54 (m, 6 H, 5-H, 6-H, 7-H), 1.68 (m, 2 H, 13-H), 1.57 (m, 2 H, 18-H' '), 1.63 (m, 2 H, 20-H), 1.661.80 (m, $6 \mathrm{H}, 18-\mathrm{H}^{\prime}$ ', $19-\mathrm{H}^{\prime}$ ', $\left.20-\mathrm{H}^{\prime}\right), 2.32\left(\mathrm{dd},{ }^{3} J_{9,8} \approx{ }^{3} J_{9,10}=7.1 \mathrm{~Hz}, 2 \mathrm{H}, 9-\mathrm{H}\right), 3.59$ (t, ${ }^{3} J_{4,5}=$ $6.8 \mathrm{~Hz}, 2 \mathrm{H}, 4-\mathrm{H}), 3.82(\mathrm{~m}, 2 \mathrm{H}, 16-\mathrm{H}), 6.24\left(\mathrm{dt},{ }^{3} J_{10,11}=15.9 \mathrm{~Hz},{ }^{3} J_{10,9}=7.2 \mathrm{~Hz}, 1 \mathrm{H}, 10-\mathrm{H}\right)$, $6.37\left(\mathrm{~d},{ }^{3} J_{11,10}=15.7 \mathrm{~Hz}, 1 \mathrm{H}, 11-\mathrm{H}\right), 7.16(\mathrm{~m}, 1 \mathrm{H}, 15-\mathrm{H}), 7.26(\mathrm{~m}, 2 \mathrm{H}, 14-\mathrm{H}), 7.32(\mathrm{~m}, 2 \mathrm{H}$, $13-\mathrm{H})$.

${ }^{13}$ C NMR (125 MHz, CDCl 3 ): $\delta=-5.3$ (q, C-3), 18.4 (s, C-2), 23.7 (bs, C-8), 25.4 (t, C-6), 25.8 (t, C-19), 26.0 (t, C-18), 26.0 (q, C-1), 26.4 (t, C-20), 27.6 (t, C-19'), 28.4 (t, C-18'), 31.0 (t, C-7), 33.2 (t, C-5), 34.8 (t, C-9), 43.1 (d, C-17), 63.4 (t, C-4), 83.4 (d, C-16), 125.9 (d, C-13), 126.6 (d, C-15), 128.4 (d, C-14), 130.2 (d, C-11), 130.7 (d, C-10), 137.9 (s, C-12).

The $Z$ isomer could not be detected.

HRMS (CI) m/z calcd for $\mathrm{C}_{34} \mathrm{H}_{58} \mathrm{O}_{3} \mathrm{BSi}[\mathrm{M}+\mathrm{H}]^{+}:$553.4243, found: 553.4240 .

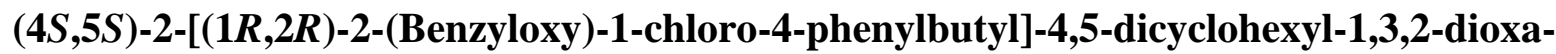 borolane (22)}

$(4 S, 5 S)-2-[(S)-1-($ Benzyloxy)-3-phenylpropyl]-4,5-dicyclohexyl-1,3,2-dioxaborolane 22 was prepared from 1 according to a slightly modified literature procedure. ${ }^{3}$

Nucleophile solution: $428 \mu \mathrm{l}(\rho=1.04 \mathrm{~g} / \mathrm{ml}, 4.11 \mathrm{mmol}, 1.4$ eq.) benzyl alcohol were added to a suspension of $153 \mathrm{mg}$ ( $3.82 \mathrm{mmol}, 1.3$ eq.) sodium hydride (60\% in mineral oil) in $1.8 \mathrm{ml}$ anhydrous THF and $5.3 \mathrm{ml}$ anhydrous DMSO. The resulting solution was stirred at room temperature for $8 \mathrm{~h}$.

Homologation and nucleophilic substitution: According to GP $11.00 \mathrm{~g}$ (2.94 mmol) boronic ester 1, $567 \mu 1(\rho=1.32 \mathrm{~g} / \mathrm{ml}, 8.82 \mathrm{mmol})$ anhydrous DCM, $2.30 \mathrm{ml}(1.6 \mathrm{M}$ in hexanes, 3.67 mmol) $n$-butyllithium, $565 \mu \mathrm{l}(\rho=0.71 \mathrm{~g} / \mathrm{ml}, 3.97 \mathrm{mmol})$ diisopropylamine and $801 \mathrm{mg}(5.88$ mmol) zinc chloride were reacted. The nucleophile solution was slowly added to the $\alpha$-chloroboronic ester solution at $0{ }^{\circ} \mathrm{C}$. After stirring at room temperature for $17 \mathrm{~h}$, the reaction was worked up and the crude product was purified by flash chromatography (petroleum ether, ethyl acetate $95: 5)$ to give 22 in $78 \%$ yield $(1.05 \mathrm{~g}, 2.28 \mathrm{mmol})$ as a pale yellow oil, $\mathrm{R}_{\mathrm{f}}=0.32$ (petroleum ether, ethyl acetate 95:5); $\alpha_{D}^{20}=-25.1\left(\mathrm{c}=1.0, \mathrm{CHCl}_{3}\right)$. Analytical data of 22 were in accordance with those previously reported. ${ }^{3}$ 


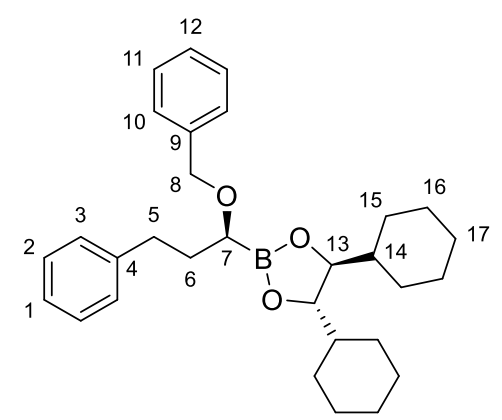

${ }^{1} \mathbf{H}$ NMR (400 MHz, $\left.\mathrm{CDCl}_{3}\right): \delta=0.99(\mathrm{~m}, 2 \mathrm{H}, 16-\mathrm{H}), 1.09(\mathrm{~m}, 2 \mathrm{H}, 15-\mathrm{H}), 1.12-1.30(\mathrm{~m}, 6 \mathrm{H}$, 15- $\left.\mathrm{H}^{\circ}, 16-\mathrm{H}^{\circ}\right), 1.35(\mathrm{~m}, 2 \mathrm{H}, 14-\mathrm{H}), 1.61\left(\mathrm{~m}, 2 \mathrm{H}, 15-\mathrm{H}^{\prime \prime}\right), 1.68(\mathrm{~m}, 2 \mathrm{H}, 17-\mathrm{H}), 1.77$ (m, $6 \mathrm{H}$, $15-\mathrm{H}^{\text {‘ }}$, $16-\mathrm{H}^{\circ}$, $\left.17-\mathrm{H}^{\circ}\right), 1.95\left(\mathrm{~m}, 1 \mathrm{H}, 6-\mathrm{H}_{\mathrm{a}}\right), 2.03\left(\mathrm{~m}, 1 \mathrm{H}, 6-\mathrm{H}_{\mathrm{b}}\right), 2.71$ (ddd, ${ }^{2} J_{5 \mathrm{a}, 5 \mathrm{~b}}=13.8 \mathrm{~Hz}$, $\left.{ }^{3} J_{5 \mathrm{a}, 6 \mathrm{a}}=10.0 \mathrm{~Hz},{ }^{3} J_{5 \mathrm{a}, 6 \mathrm{~b}}=6.6 \mathrm{~Hz}, 1 \mathrm{H}, 5-\mathrm{H}_{\mathrm{a}}\right), 2.80\left(\mathrm{ddd},{ }^{2} J_{5 \mathrm{~b}, 5 \mathrm{a}}=13.7 \mathrm{~Hz},{ }^{3} J_{5 \mathrm{~b}, 6 \mathrm{a}}=10.0 \mathrm{~Hz}\right.$, $\left.{ }^{3} J_{5 \mathrm{~b}, 6 \mathrm{~b}}=5.5 \mathrm{~Hz}, 1 \mathrm{H}, 5-\mathrm{H}_{\mathrm{b}}\right), 3.39\left(\mathrm{dd},{ }^{3} J_{7,6 \mathrm{~b}}=8.1 \mathrm{~Hz},{ }^{3} J_{7,6 \mathrm{a}}=5.4 \mathrm{~Hz}, 1 \mathrm{H}, 7-\mathrm{H}\right), 3.92(\mathrm{~m}, 2 \mathrm{H}$, $13-\mathrm{H}), 4.49\left(\mathrm{~d},{ }^{2} J_{8 \mathrm{a}, 8 \mathrm{~b}}=11.7 \mathrm{~Hz}, 1 \mathrm{H}, 8-\mathrm{H}_{\mathrm{a}}\right), 4.63\left(\mathrm{~d},{ }^{2} J_{8 \mathrm{~b}, 8 \mathrm{a}}=11.9 \mathrm{~Hz}, 1 \mathrm{H}, 8-\mathrm{H}_{\mathrm{b}}\right), 7.13-7.20$ (m, 3 H, 1-H, 3-H), 7.24-7.30 (m, 3 H, 2-H, 12-H), 7.34 (m, 2 H, 11-H), 7.39 (m, 2 H, 10-H).

${ }^{13} \mathrm{C}$ NMR $\left(100 \mathrm{MHz}, \mathrm{CDCl}_{3}\right): \delta=25.9(\mathrm{t}, \mathrm{C}-16), 26.0(\mathrm{t}, \mathrm{C}-15), 26.4(\mathrm{t}, \mathrm{C}-17), 27.4\left(\mathrm{t}, \mathrm{C}-16^{\circ}\right)$, 28.2 (t, C-15'), 32.8 (t, C-5), 33.5 (t, C-6), 42.9 (d, C-14), 72.3 (t, C-8), 83.7 (d, C-13), 125.6 (d, C-1), 127.4 (d, C-12), 128.0 (d, C-10), 128.2 (d, C-2/3/11), 128.3 (d, C-2/3/11), 128.6 (d, C-2/3/11), 139.1 (s, C-9), 142.5 (s, C-4).

\section{(4S,5S)-2-[(1R,2R)-2-(Benzyloxy)-1-chloro-4-phenylbutyl]-4,5-dicyclohexyl-1,3,2-dioxa- borolane (23)}

According to GP 1a $986 \mathrm{mg}(2.14 \mathrm{mmol}) 22,413 \mu \mathrm{l}(\rho=1.32 \mathrm{~g} / \mathrm{ml}, 6.42 \mathrm{mmol})$ anhydrous DCM, $1.67 \mathrm{ml}(1.6 \mathrm{M}$ in hexanes, $2.68 \mathrm{mmol}) n$-butyllithium, $412 \mu \mathrm{l}(\rho=0.71 \mathrm{~g} / \mathrm{ml}, 2.89 \mathrm{mmol})$ diisopropylamine and $875 \mathrm{mg}(6.42 \mathrm{mmol})$ zinc chloride were reacted. After stirring at room temperature for $3 \mathrm{~h}$, the reaction was worked up to obtain crude 23 in $96 \%$ yield $(1.05 \mathrm{~g}$, $2.06 \mathrm{mmol}$ ) as a yellow solid, m.p. $69^{\circ} \mathrm{C}$ (from pentane, THF); $\alpha_{D}^{20}=-24.4\left(\mathrm{c}=0.5, \mathrm{CHCl}_{3}\right)$. $\mathbf{2 3}$ was used in the next step without further purification.

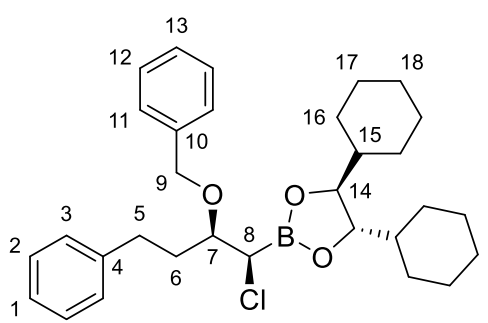

${ }^{1} \mathbf{H}$ NMR $\left(500 \mathrm{MHz}, \mathrm{CDCl}_{3}\right): \delta=0.98(\mathrm{~m}, 2 \mathrm{H}, 17-\mathrm{H}), 1.05(\mathrm{~m}, 2 \mathrm{H}, 16-\mathrm{H}), 1.09-1.25(\mathrm{~m}, 6 \mathrm{H}$, 16-H', 17-H'), 1.35 (m, 2 H, 15-H), 1.61 (m, 2 H, 16-H' '), 1.66 (m, 2 H, 18-H), 1.70-1.81 (m, $\left.6 \mathrm{H}, 16-\mathrm{H}^{\prime}, ', 17-\mathrm{H}^{\prime},, 18-\mathrm{H}^{\prime}\right), 1.94$ (dddd, ${ }^{2} J_{6 \mathrm{a}, 6 \mathrm{~b}}=14.0 \mathrm{~Hz},{ }^{3} J_{6 \mathrm{a}, 5 \mathrm{a}}=10.3 \mathrm{~Hz},{ }^{3} J_{6 \mathrm{a}, 7}=7.7 \mathrm{~Hz}$, ${ }^{3} J_{6 \mathrm{a}, 5 \mathrm{~b}}=5.4 \mathrm{~Hz}, 1 \mathrm{H}, 6-\mathrm{H}_{\mathrm{a}}$ ), 2.07 (dddd, ${ }^{2} J_{6 \mathrm{~b}, 6 \mathrm{a}}=14.2 \mathrm{~Hz},{ }^{3} J_{5 \mathrm{~b}, 6 \mathrm{~b}}=10.3 \mathrm{~Hz},{ }^{3} J_{6 \mathrm{~b}, 5 \mathrm{a}}=6.2 \mathrm{~Hz}$, $\left.{ }^{3} J_{6 \mathrm{~b}, 7}=4.3 \mathrm{~Hz}, 1 \mathrm{H}, 6-\mathrm{H}_{\mathrm{b}}\right), 2.68\left(\mathrm{ddd},{ }^{2} J_{5 \mathrm{a}, 5 \mathrm{~b}}=13.7 \mathrm{~Hz},{ }^{3} J_{5 \mathrm{a}, 6 \mathrm{a}}=10.2 \mathrm{~Hz},{ }^{3} J_{5 \mathrm{a}, 6 \mathrm{~b}}=6.1 \mathrm{~Hz}, 1 \mathrm{H}\right.$, $\left.5-\mathrm{H}_{\mathrm{a}}\right), 2.77\left(\mathrm{ddd},{ }^{2} J_{5 \mathrm{~b}, 5 \mathrm{a}}=13.9 \mathrm{~Hz},{ }^{3} J_{5 \mathrm{~b}, 6 \mathrm{~b}}=10.5 \mathrm{~Hz},{ }^{3} J_{5 \mathrm{~b}, 6 \mathrm{a}}=5.5 \mathrm{~Hz}, 1 \mathrm{H}, 5-\mathrm{H}_{\mathrm{b}}\right), 3.69\left(\mathrm{~d},{ }^{3} J_{8,7}=\right.$ $6.9 \mathrm{~Hz}, 1 \mathrm{H}, 8-\mathrm{H}), 3.80\left(\mathrm{ddd},{ }^{3} J_{7,6 \mathrm{a}} \approx{ }^{3} J_{7,8}=7.2 \mathrm{~Hz},{ }^{3} J_{7,6 \mathrm{~b}}=4.3 \mathrm{~Hz}, 1 \mathrm{H}, 7-\mathrm{H}\right), 3.95(\mathrm{~m}, 2 \mathrm{H}$, $14-\mathrm{H}), 4.60\left(\mathrm{~d},{ }^{2} J_{9 \mathrm{a}, 9 \mathrm{~b}}=11.4 \mathrm{~Hz}, 1 \mathrm{H}, 9-\mathrm{H}_{\mathrm{a}}\right), 4.73\left(\mathrm{~d},{ }^{2} J_{9 \mathrm{~b}, 9 \mathrm{a}}=11.3 \mathrm{~Hz}, 1 \mathrm{H}, 9-\mathrm{H}_{\mathrm{b}}\right), 7.13-7.21$ (m, 3 H, 1-H, 3-H), 7.24-7.31 (m, 3 H, 2-H, 13-H), 7.34 (m, 2 H, 12-H), 7.39 (m, 2 H, 11-H).

${ }^{13} \mathrm{C}$ NMR $\left(125 \mathrm{MHz}, \mathrm{CDCl}_{3}\right): \delta=25.8(\mathrm{t}, \mathrm{C}-17), 25.9$ (t, C-16), $16.3(\mathrm{t}, \mathrm{C}-18), 27.4$ (t, C-17'), 28.2 (t, C-16'), 31.6 (t, C-5), 33.8 (t, C-6), 42.8 (d, C-15), 72.6 (t, C-9), 80.0 (d, C-7), 84.2 (d, C-14), 125.8 (d, C-1), 127.5 (d, C-13), 127.7 (d, C-11), 128.3 (d, C-2/3/12), 128.3 (d, C-2/3/12), 128.4 (d, C-2/3/12), 138.5 (s, C-10), 141.8 (s, C-4).

The signal of C-8 could not be detected. 
HRMS (CI) m/z calcd for $\mathrm{C}_{31} \mathrm{H}_{43} \mathrm{O}_{3} \mathrm{BCl}[\mathrm{M}+\mathrm{H}]^{+}:$509.2988, found: 509.2999.

\section{(4S,5S)-2-[(4R,5R,E)-5-(Benzyloxy)-1,7-diphenylhept-1-en-4-yl]-4,5-dicyclohexyl-1,3,2- dioxaborolane (24)}

According to GP $496 \mathrm{mg}(189 \mu \mathrm{mol}) \alpha$-chloroboronic ester 23 were reacted with $1.02 \mathrm{ml}$ $(377 \mu \mathrm{mol})$ allylzinc reagent $9 \mathrm{~g}(0.37 \mathrm{M}$ in THF). After stirring for $18 \mathrm{~h}$, the reaction was worked up and the crude product was purified by flash chromatography (petroleum ether, ethyl acetate 98:2) to give $\mathbf{2 4}\left(E: Z>96: 4\right.$ according to $\left.{ }^{1} \mathrm{H} \mathrm{NMR}\right)$ in $87 \%$ yield $(97 \mathrm{mg}, 164 \mu \mathrm{mol})$ as a colorless oil, $\mathrm{R}_{\mathrm{f}}=0.14$ (petroleum ether, ethyl acetate 98:2); $\alpha_{D}^{20}=-20.5\left(\mathrm{c}=0.5, \mathrm{CHCl}_{3}\right)$. An analytically pure sample of $\mathbf{2 4}$ was obtained by reversed-phase flash chromatography $\left(\mathrm{H}_{2} \mathrm{O}\right.$, $70 \% \mathrm{MeCN} \rightarrow 100 \% \mathrm{MeCN})$.

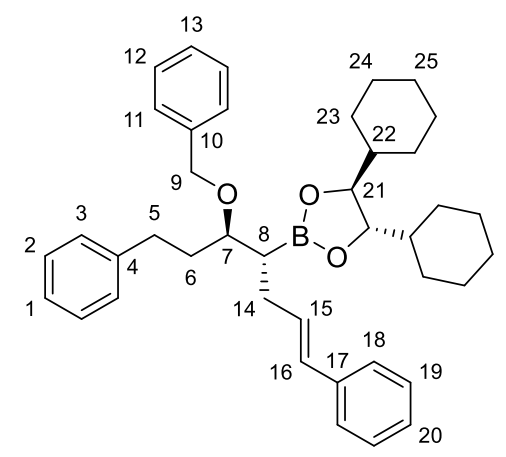

${ }^{1} \mathbf{H}$ NMR (500 MHz, $\left.\mathrm{CDCl}_{3}\right): \delta=0.92(\mathrm{~m}, 2 \mathrm{H}, 24-\mathrm{H}), 1.00(\mathrm{~m}, 2 \mathrm{H}, 23-\mathrm{H}), 1.04-1.18(\mathrm{~m}, 6 \mathrm{H}$, 23-H', 24-H'), 1.25 (m, 2 H, 22-H), 1.56 (m, 2 H, 23-H'), 1.59-1.81 (m, 9 H, 8-H, 23-H', ', 24-H', 25-H), $1.98(\mathrm{~m}, 2 \mathrm{H}, 6-\mathrm{H}), 2.42(\mathrm{~m}, 2 \mathrm{H}, 14-\mathrm{H}), 2.67\left(\mathrm{ddd}, J_{5 \mathrm{a}, 5 \mathrm{~b}}=13.7 \mathrm{~Hz},{ }^{3} J_{5 \mathrm{a}, 6 \mathrm{a}}=\right.$ $\left.9.8 \mathrm{~Hz},{ }^{3} J_{5 \mathrm{a}, 6 \mathrm{~b}}=6.3 \mathrm{~Hz}, 1 \mathrm{H}, 5-\mathrm{H}_{\mathrm{a}}\right), 2.79\left(\mathrm{ddd},{ }^{2} J_{5 \mathrm{~b}, 5 \mathrm{a}}=13.8 \mathrm{~Hz},{ }^{3} J_{5 \mathrm{~b}, 6 \mathrm{~b}}=10.1 \mathrm{~Hz},{ }^{3} J_{5 \mathrm{~b}, 6 \mathrm{a}}=5.7 \mathrm{~Hz}\right.$, $\left.1 \mathrm{H}, 5-\mathrm{H}_{\mathrm{b}}\right), 3.65\left(\mathrm{ddd},{ }^{3} J_{7,6 \mathrm{a}} \approx{ }^{3} J_{7,8}=7.2 \mathrm{~Hz},{ }^{3} J_{7,6 \mathrm{~b}}=4.6 \mathrm{~Hz}, 1 \mathrm{H}, 7-\mathrm{H}\right), 3.82(\mathrm{~m}, 2 \mathrm{H}, 21-\mathrm{H})$, $4.51\left(\mathrm{~d},{ }^{2} J_{9 \mathrm{a}, 9 \mathrm{~b}}=11.7 \mathrm{~Hz}, 1 \mathrm{H}, 9-\mathrm{H}_{\mathrm{a}}\right), 4.57\left(\mathrm{~d},{ }^{2} J_{9 \mathrm{~b}, 9 \mathrm{a}}=11.7 \mathrm{~Hz}, 1 \mathrm{H}, 9-\mathrm{H}_{\mathrm{b}}\right), 6.26\left(\mathrm{dt},{ }^{3} J_{15,16}=\right.$ $\left.15.7 \mathrm{~Hz},{ }^{3} J_{15,14}=6.9 \mathrm{~Hz}, 1 \mathrm{H}, 15-\mathrm{H}\right), 6.35\left(\mathrm{~d},{ }^{3} J_{16,15}=15.7 \mathrm{~Hz}, 1 \mathrm{H}, 16-\mathrm{H}\right), 7.12-7.40(\mathrm{~m}, 15 \mathrm{H}$, 1-H, 2-H, 3-H, 11-H, 12-H, 13-H, 18-H, 19-H, 20-H).

${ }^{13}$ C NMR (125 MHz, $\left.\mathrm{CDCl}_{3}\right): \delta=25.8$ (t, C-24), 25.9 (t, C-23), 26.4 (t, C-25), 27.7 (t, C-24'), 28.3 (bs, C-8), 28.5 (t, C-23'), 30.1 (t, C-14), 32.1 (t, C-5), 34.6 (t, C-6), 43.0 (d, C-22), 70.8 (t, C-9), 79.9 (d, C-7), 83.5 (d, C-21), 125.6 (d, C-1), 125.9 (d, C-18), 126.7 (d, C-20), 127.2 (d, C-13), 127.5 (d, C-11), 128.2 (d, C-2/3/12/19), 128.3 (d, C-2/3/12/19), 128.4 (d, C-2/3/12/19), 128.4 (d, C-2/3/12/19), 130.2 (d, C-16), 130.9 (d, C-15), 137.9 (s, C-17), 139.2 (s, C-10), 142.6 (s, C-4).

The $Z$ isomer could not be detected.

HRMS (CI) m/z calcd for $\mathrm{C}_{40} \mathrm{H}_{52} \mathrm{O}_{3} \mathrm{~B}[\mathrm{M}+\mathrm{H}]^{+}:$591.4004, found: 591.4000 .

\section{(4S,5S)-2-[(S)-2-(Benzyloxy)-4-phenylbutyl]-4,5-dicyclohexyl-1,3,2-dioxaborolane (23-H)}

$1.51 \mathrm{ml}\left(1.51 \mathrm{mmol}, 1.1\right.$ eq.) $\mathrm{LiBHEt}_{3}(1.0 \mathrm{M}$ in THF) were added dropwise to a solution of $700 \mathrm{mg}$ ( $1.38 \mathrm{mmol}, 1.0$ eq.) $\alpha$-chloroboronic ester $23 \mathrm{in} 3.4 \mathrm{ml}$ anhydrous THF at $0{ }^{\circ} \mathrm{C}$. The mixture was allowed to warm to room temperature and stirred for $27 \mathrm{~h}$. Saturated $\mathrm{NH}_{4} \mathrm{Cl}$ solution was added and the layers were separated. The aqueous layer was extracted twice with pentane. The combined organic layers were dried over $\mathrm{Na}_{2} \mathrm{SO}_{4}$ and concentrated in vacuo. Purification of the residue by flash chromatography (petroleum ether, ethyl acetate 95:5) gave 23-H in $90 \%$ yield $(590 \mathrm{mg}, 1.24 \mathrm{mmol})$ as a colorless oil, $\mathrm{R}_{\mathrm{f}}=0.29$ (petroleum ether, ethyl acetate 95:5); $\alpha_{D}^{20}=-17.1\left(\mathrm{c}=0.5, \mathrm{CHCl}_{3}\right)$. 


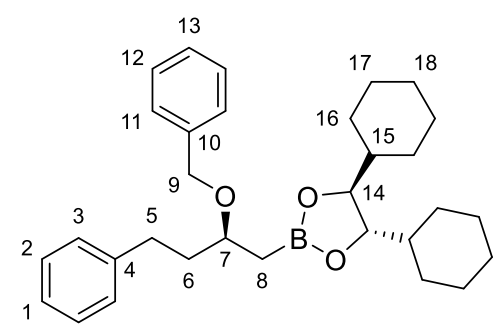

${ }^{1} \mathbf{H}$ NMR $\left(500 \mathrm{MHz}, \mathrm{CDCl}_{3}\right): \delta=0.97(\mathrm{~m}, 2 \mathrm{H}, 17-\mathrm{H}), 1.06(\mathrm{~m}, 2 \mathrm{H}, 16-\mathrm{H}), 1.10-1.25(\mathrm{~m}, 7 \mathrm{H}$, $\left.8-\mathrm{H}_{\mathrm{a}}, 16-\mathrm{H}^{\prime}, 17-\mathrm{H}^{\prime}\right), 1.31(\mathrm{~m}, 2 \mathrm{H}, 15-\mathrm{H}), 1.36\left(\mathrm{dd},{ }^{2} J_{8 \mathrm{~b}, 8 \mathrm{a}}=15.1 \mathrm{~Hz},{ }^{3} J_{8,7}=5.4 \mathrm{~Hz}, 1 \mathrm{H}, 8-\mathrm{H}_{\mathrm{a}}\right)$, 1.59 (m, 2 H, 16-H' '), 1.67 (m, 2 H, 18-H), 1.71-1.82 (m, 6 H, 16-H' ', 17-H', 18-H'), 1.93 $(\mathrm{m}, 2 \mathrm{H}, 6-\mathrm{H}), 2.68\left(\mathrm{ddd},{ }^{2} J_{5 \mathrm{a}, 5 \mathrm{~b}}=13.9 \mathrm{~Hz},{ }^{3} J_{5 \mathrm{a}, 6 \mathrm{a}}=9.5 \mathrm{~Hz},{ }^{3} J_{5 \mathrm{a}, 6 \mathrm{~b}}=7.3 \mathrm{~Hz}, 1 \mathrm{H}, 5-\mathrm{H}_{\mathrm{a}}\right), 2.80$ $\left(\mathrm{ddd},{ }^{2} J_{5 \mathrm{~b}, 5 \mathrm{a}}=14.2 \mathrm{~Hz},{ }^{3} J_{5 \mathrm{~b}, 6 \mathrm{~b}}=9.5 \mathrm{~Hz},{ }^{3} J_{5 \mathrm{~b}, 6 \mathrm{a}}=6.3 \mathrm{hz}, 1 \mathrm{H}, 5-\mathrm{H}_{\mathrm{b}}\right), 3.72(\mathrm{~m}, 1 \mathrm{H}, 7-\mathrm{H}), 3.84(\mathrm{~m}$, $2 \mathrm{H}, 14-\mathrm{H}), 4.47\left(\mathrm{~d},{ }^{2} J_{9 \mathrm{a}, 9 \mathrm{~b}}=11.7 \mathrm{~Hz}, 1 \mathrm{H}, 9-\mathrm{H}_{\mathrm{a}}\right), 4.61\left(\mathrm{~d},{ }^{2} J_{9 \mathrm{~b}, 9 \mathrm{a}}=11.7 \mathrm{~Hz}, 1 \mathrm{H}, 9-\mathrm{H}_{\mathrm{a}}\right), 7.14-$ 7.20 (m, 3 H, 1-H, 3-H), 7.24-7.29 (sh, 3 H, 2-H, 13-H), 7.33 (m, 2 H, 12-H), 7.37 (m, 2 H, 11-H).

${ }^{13} \mathrm{C}$ NMR $\left(125 \mathrm{MHz}, \mathrm{CDCl}_{3}\right): \delta=16.9$ (bs, C-8), 25.8 (t, C-17), 26.0 (t, C-16), 26.4 (t, C-18), 27.4 (t, C-17'), 28.4 (t, C-16'), 23.7 (t, C-5), 38.0 (t, C-6), 43.0 (d, C-15), 70.4 (t, C-9), 76.1 (d, C-7), 83.4 (d, C-14), 125.5 (d, C-1), 127.3 (d, C-13), 127.6 (d, C-11), 128.2 (d, C-2, C-12), 128.4 (d, C-3), 139.2 (s, C-10), 142.6 (s, C-4).

HRMS (CI) m/z calcd for $\mathrm{C}_{31} \mathrm{H}_{43} \mathrm{O}_{3} \mathrm{~B}[\mathrm{M}]^{+}:$474.3300, found: 474.3331 .

\section{(4S,5S)-2-[(1R,3R)-3-(Benzyloxy)-1-chloro-5-phenylpentyl]-4,5-dicyclohexyl-1,3,2- dioxaborolane (25)}

According to GP 1a $562 \mathrm{mg}(1.18 \mathrm{mmol}) \mathbf{2 3 - H}, 229 \mu 1(\rho=1.32 \mathrm{~g} / \mathrm{ml}, 3.55 \mathrm{mmol})$ anhydrous DCM, $925 \mu \mathrm{l}(1.6 \mathrm{M}$ in hexanes, $1.48 \mathrm{mmol}) n$-butyllithium, $228 \mu \mathrm{l}(\rho=0.71 \mathrm{~g} / \mathrm{ml}, 1.60 \mathrm{mmol})$ diisopropylamine and $484 \mathrm{mg}$ (3.55 mmol) zinc chloride were reacted. After stirring at room temperature for $2 \mathrm{~h}$, the reaction was worked up to obtain crude 25 in $96 \%$ yield (598 mg, $1.14 \mathrm{mmol})$ as a yellow oil, $\alpha_{D}^{20}=-52.4\left(\mathrm{c}=0.5, \mathrm{CHCl}_{3}\right) .25$ was used in the next step without further purification.

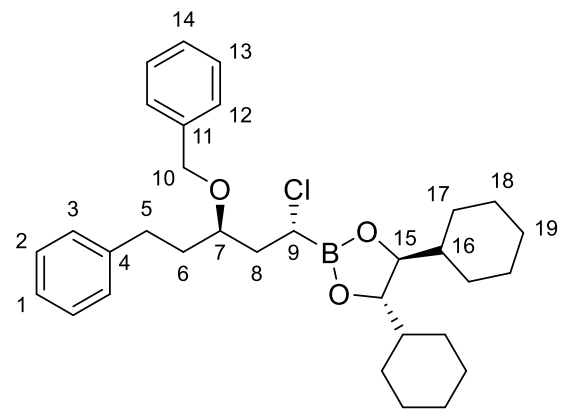

${ }^{1} \mathbf{H}$ NMR $\left(500 \mathrm{MHz}, \mathrm{CDCl}_{3}\right): \delta=0.98(\mathrm{~m}, 2 \mathrm{H}, 18-\mathrm{H}), 1.07(\mathrm{~m}, 2 \mathrm{H}, 17-\mathrm{H}), 1.12-1.30(\mathrm{~m}, 6 \mathrm{H}$, 17-H', 18-H'), 1.35 (m, 2 H, 16-H), 1.60 (m, 2 H, 17-H' '), 1.68 (m, 2 H, 19-H), 1.71-1.82 (m, $\left.6 \mathrm{H}, 17-\mathrm{H}^{\prime}, ', 18-\mathrm{H}^{\prime}, 19-\mathrm{H}^{\prime}\right), 1.87-2.04\left(\mathrm{~m}, 3 \mathrm{H}, 6-\mathrm{H}, 8-\mathrm{H}_{\mathrm{a}}\right), 2.10\left(\mathrm{ddd},{ }^{2} J_{8 \mathrm{a}, 8 \mathrm{~b}}=14.5 \mathrm{~Hz},{ }^{3} J_{8 \mathrm{~b}, 7}=\right.$ $\left.9.1 \mathrm{~Hz},{ }^{3} J_{8 \mathrm{~b}, 9}=4.1 \mathrm{~Hz}, 1 \mathrm{H}, 8-\mathrm{H}_{\mathrm{b}}\right), 2.69\left(\mathrm{t},{ }^{3} J_{5,6}=8.2 \mathrm{~Hz}, 2 \mathrm{H}, 5-\mathrm{H}\right), 3.78\left(\mathrm{dd},{ }^{3} J_{9,8 \mathrm{a}}=11.0 \mathrm{~Hz}\right.$, $\left.{ }^{3} J_{9,8 \mathrm{~b}}=4.1 \mathrm{~Hz}, 1 \mathrm{H}, 8-\mathrm{H}_{\mathrm{b}}\right), 3.82(\mathrm{~m}, 1 \mathrm{H}, 7-\mathrm{H}), 3.94(\mathrm{~m}, 2 \mathrm{H}, 15-\mathrm{H}), 4.55\left(\mathrm{~d},{ }^{2} J_{10 \mathrm{a}, 10 \mathrm{~b}}=11.3 \mathrm{~Hz}\right.$, $1 \mathrm{H}, 10-\mathrm{H}), 4.62\left(\mathrm{~d},{ }^{3} J_{10 \mathrm{~b}, 10 \mathrm{a}}=11.3 \mathrm{~Hz}, 1 \mathrm{H}, 10-\mathrm{H}_{\mathrm{b}}\right), 7.14-7.22(\mathrm{~m}, 3 \mathrm{H}, 1-\mathrm{H}, 3-\mathrm{H}), 7.26-7.32$ (m, $3 \mathrm{H}, 2-\mathrm{H}, 14-\mathrm{H}), 7.32-7.41$ (m, $4 \mathrm{H}, 12-\mathrm{H}, 13-\mathrm{H})$.

${ }^{13} \mathrm{C}$ NMR $\left(125 \mathrm{MHz}, \mathrm{CDCl}_{3}\right): \delta=25.8(\mathrm{t}, \mathrm{C}-18), 25.9$ (t, C-17), $26.4(\mathrm{t}, \mathrm{C}-19), 27.3(\mathrm{t}, \mathrm{C}-18$ '), 28.2 (t, C-17'), 31.3 (t, C-5), 35.8 (t, C-6), 39.0 (t, C-8), 39.8 (bs, C-9), 42.8 (d, C-16), 71.8 (t, 
C-10), 75.2 (d, C-7), 84.1 (d, C-18), 125.8 (d, C-1), 127.6 (d, C-14), 128.0 (d, C-12), 128.3 (d, C-1/2/13), 128.4 (d, C-1/2/13), 128.4 (d, C-1/2/13), 138.6 (s, C-11), 142.2 (s, C-4).

HRMS $(\mathrm{CI}) \mathrm{m} / \mathrm{z}$ calcd for $\mathrm{C}_{32} \mathrm{H}_{45} \mathrm{O}_{3} \mathrm{BCl}[\mathrm{M}+\mathrm{H}]^{+}:$523.3145, found: 523.3138 .

\section{$(4 S, 5 S)-2-[(4 R, 6 R, E)-6-(B e n z y l o x y)-1,8-d i p h e n y l o c t-1-e n-4-y l]-4,5-d i c y c l o h e x y l-1,3,2-$ dioxaborolane (26)}

According to GP $4100 \mathrm{mg}(191 \mu \mathrm{mol}) \alpha$-chloroboronic ester 25 were reacted with $1.03 \mathrm{ml}$ $(381 \mu \mathrm{mol})$ allylzinc reagent $\mathbf{9 g}(0.37 \mathrm{M}$ in THF). After stirring for $14 \mathrm{~h}$, the reaction was worked up and the crude product was purified by flash chromatography (petroleum ether, ethyl acetate 98:2) to give $\mathbf{2 6}\left(E: Z>96: 4\right.$ according to $\left.{ }^{1} \mathrm{H} \mathrm{NMR}\right)$ in $75 \%$ yield $(86 \mathrm{mg}, 142 \mu \mathrm{mol})$ as a colorless oil, $\mathrm{R}_{\mathrm{f}}=0.07$ (petroleum ether, ethyl acetate 98:2); $\alpha_{D}^{20}=-21.8\left(\mathrm{c}=0.5, \mathrm{CHCl}_{3}\right)$. An analytically pure sample of $\mathbf{2 6}$ was obtained by reversed-phase flash chromatography $\left(\mathrm{H}_{2} \mathrm{O}\right.$, $70 \% \mathrm{MeCN} \rightarrow 100 \% \mathrm{MeCN})$.

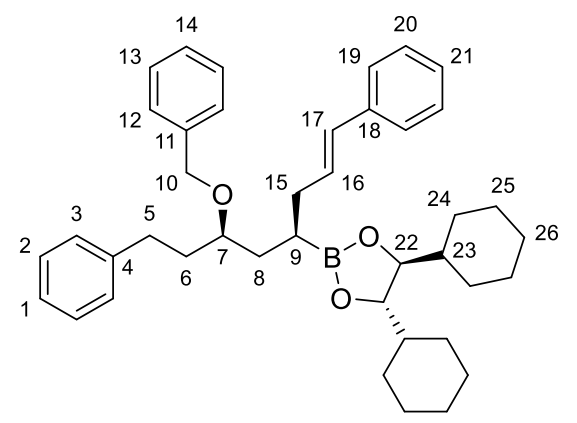

${ }^{1} \mathbf{H}$ NMR $\left(500 \mathrm{MHz}, \mathrm{CDCl}_{3}\right): \delta=0.94(\mathrm{~m}, 2 \mathrm{H}, 25-\mathrm{H}), 1.00$ (m, $\left.2 \mathrm{H}, 24-\mathrm{H}\right), 1.05-1.39(\mathrm{~m}, 6 \mathrm{H}$, 23-H, 24-H', 25-H'), 1.25 (m, 2 H, 22-H), 1.46-1.58 (m, 3 H, 9-H, 24-H' '), 1.59-1.81 (m, $10 \mathrm{H}, 8-\mathrm{H}, 23-\mathrm{H}$ ', $24-\mathrm{H}$ ', $25-\mathrm{H}), 1.87$ (m, $2 \mathrm{H}, 6-\mathrm{H}), 2.33\left(\mathrm{dd},{ }^{3} J_{15,9} \approx{ }^{3} J_{15,16}=7.1 \mathrm{~Hz}, 2 \mathrm{H}\right.$, $15-\mathrm{H}), 2.64\left(\mathrm{ddd},{ }^{2} J_{5 \mathrm{a}, 5 \mathrm{~b}}=13.6 \mathrm{~Hz},{ }^{3} J_{5 \mathrm{a}, 6 \mathrm{a}}=10.5 \mathrm{~Hz},{ }^{3} J_{5 \mathrm{a}, 6 \mathrm{~b}}=6.3 \mathrm{~Hz}, 1 \mathrm{H}, 5-\mathrm{H}_{\mathrm{a}}\right), 2.72(\mathrm{ddd}$, $\left.{ }^{2} J_{5 \mathrm{~b}, 5 \mathrm{a}}=13.7 \mathrm{~Hz},{ }^{3} J_{5 \mathrm{~b}, 6 \mathrm{~b}}=10.5 \mathrm{~Hz},{ }^{3} J_{5 \mathrm{~b}, 6 \mathrm{a}}=5.8 \mathrm{~Hz}, 1 \mathrm{H}, 5-\mathrm{H}_{\mathrm{b}}\right), 3.53\left(\mathrm{tt},{ }^{3} J_{7,6} \approx{ }^{3} J_{7,8}=6.0 \mathrm{~Hz}\right.$, $1 \mathrm{H}, 7-\mathrm{H}), 3.79(\mathrm{~m}, 2 \mathrm{H}, 22-\mathrm{H}), 4.52\left(\mathrm{~d},{ }^{2} J_{10 \mathrm{a}, 10 \mathrm{~b}}=11.7 \mathrm{~Hz}, 1 \mathrm{H}, 10-\mathrm{H}_{\mathrm{a}}\right), 4.55\left(\mathrm{~d},{ }^{2} J_{10 \mathrm{~b}, 10 \mathrm{a}}=\right.$ $\left.11.6 \mathrm{~Hz}, 1 \mathrm{H}, 10-\mathrm{H}_{\mathrm{b}}\right), 6.24\left(\mathrm{dt},{ }^{3} J_{16,17}=15.7 \mathrm{~Hz},{ }^{3} J_{16,15}=7.2 \mathrm{~Hz}, 1 \mathrm{H}, 16-\mathrm{H}\right), 6.37\left(\mathrm{~d},{ }^{3} J_{17,16}=\right.$ $15.9 \mathrm{~Hz}, 1 \mathrm{H}, 17-\mathrm{H}$ ), 7.14-7.39 (sh, 15 H, 1-H, 2-H, 3-H, 12-H, 13-H, 14-H, 19-H, 20-H, 21-H).

${ }^{13} \mathrm{C}$ NMR (125 MHz, $\left.\mathrm{CDCl}_{3}\right): \delta=19.9$ (bs, C-9), 25.8 (t, C-25), 25.9 (t, C-24), 26.4 (t, C-26), 27.7 (t, C-25'), 28.6 (t, C-24'), 31.7 (t, C-5), 35.4 (t, C-15), 35.9 (t, C-8), 36.1 (t, C-6), 43.0 (d, C-23), 70.9 (t, C-10), 78.3 (d, C-7), 83.5 (d, C-22), 125.7 (d, C-1), 126.0 (d, C-19), 126.7 (d, C-21), 127.4 (d, C-14), 127.8 (d, C-12), 128.3 (d, C-2/3/13/20), 128.3 (d, C-2/3/13/20), 128.4 (d, C-2/3/13/20), 130.4 (d, C-17), 130.5 (d, C-16), 137.8 (s, C-18), 139.0 (s, C-11), 142.6 (s, C-4).

The $Z$ isomer could not be detected.

HRMS (CI) m/z calcd for $\mathrm{C}_{41} \mathrm{H}_{54} \mathrm{O}_{3} \mathrm{~B}[\mathrm{M}+\mathrm{H}]^{+}$: 605.4161, found: 605.4167.

\section{Matteson homologation and modification of $\alpha$-allylated boronic esters}

\section{(4S,5S)-4,5-Dicyclohexyl-2-[(3R,4S,E)-4-phenethyl-7-phenylhept-6-en-3-yl]-1,3,2-dioxa-} borolane (27)

According to GP $1 \mathrm{~b} 415 \mathrm{mg}(882 \mu \mathrm{mol})$ boronic ester $10 \mathrm{~g}, 170 \mu \mathrm{l}(\rho=1.32 \mathrm{~g} / \mathrm{ml}, 2.64 \mathrm{mmol})$ anhydrous DCM, $689 \mu \mathrm{l}(1.6 \mathrm{M}$ in hexanes, $1.10 \mathrm{mmol}) n$-butyllithium, $170 \mu 1(\rho=0.71 \mathrm{~g} / \mathrm{ml}$, $1.19 \mathrm{mmol})$ diisopropylamine and $240 \mathrm{mg}(1.76 \mathrm{mmol})$ zinc chloride were reacted. To the $\alpha-$ chloroboronic ester solution were added $735 \mu 1$ (2.21 mmol, 2.5 eq.) ethylmagnesium bromide (3.0 $\mathrm{M}$ in diethyl ether) at $0{ }^{\circ} \mathrm{C}$. After stirring at room temperature for $2 \mathrm{~d}$, the reaction was 
worked up and the crude product was purified by flash chromatography (petroleum ether, ethyl acetate $98: 2)$ to give 27 in $83 \%$ yield $(375 \mathrm{mg}, 732 \mu \mathrm{mol})$ as a colorless oil, $\mathrm{R}_{\mathrm{f}}=0.18$ (petroleum ether, ethyl acetate 98:2); $\alpha_{D}^{20}=-54.3\left(\mathrm{c}=0.5, \mathrm{CHCl}_{3}\right)$. An analytically pure sample of 21 was obtained by reversed-phase flash chromatography $\left(\mathrm{H}_{2} \mathrm{O}, 70 \% \mathrm{MeCN} \rightarrow 100 \%\right.$ $\mathrm{MeCN})$.

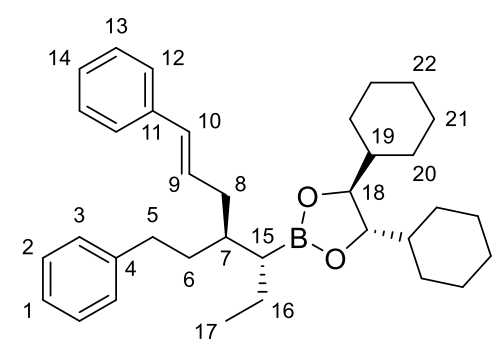

${ }^{1} \mathbf{H}$ NMR $\left(500 \mathrm{MHz}, \mathrm{CDCl}_{3}\right): \delta=0.93\left(\mathrm{t},{ }^{3} J_{17,16}=7.3 \mathrm{~Hz}, 3 \mathrm{H}, 17-\mathrm{H}\right), 1.00(\mathrm{~m}, 2 \mathrm{H}, 21-\mathrm{H}), 1.07$ (m, 2 H, 21-H), 1.11-1.35 (m, 9 H, 15-H, 19-H, 20-H', 21-H'), 1.45 (m, $\left.1 \mathrm{H}, 16-\mathrm{H}_{\mathrm{a}}\right), 1.53$ (m, $\left.1 \mathrm{H}, 16-\mathrm{H}_{\mathrm{b}}\right), 1.60$ (m, $2 \mathrm{H}, 20-\mathrm{H}^{\prime}$ '), 1.64-1.80 (m, $11 \mathrm{H}, 6-\mathrm{H}, 7-\mathrm{H}, 20-\mathrm{H}^{\prime}$ ', $\left.21-\mathrm{H}^{\prime},, 22-\mathrm{H}\right), 2.21$ $\left(\mathrm{ddd},{ }^{2} J_{8 \mathrm{a}, 8 \mathrm{~b}}=14.4 \mathrm{~Hz},{ }^{3} J_{8 \mathrm{a}, 7} \approx{ }^{3} J_{8 \mathrm{a}, 9}=7.4 \mathrm{~Hz}, 1 \mathrm{H}, 8-\mathrm{H}_{\mathrm{a}}\right), 2.37\left(\mathrm{ddd},{ }^{2} J_{8 \mathrm{~b}, 8 \mathrm{a}}=14.2 \mathrm{~Hz},{ }^{3} J_{8 \mathrm{~b}, 7} \approx\right.$ $\left.{ }^{3} J_{8 \mathrm{~b}, 9}=5.7 \mathrm{~Hz}, 1 \mathrm{H}, 8-\mathrm{H}_{\mathrm{b}}\right), 2.59\left(\mathrm{dt},{ }^{2} J_{5 \mathrm{a}, 5 \mathrm{~b}}=13.9 \mathrm{~Hz},{ }^{3} J_{5 \mathrm{a}, 6}=8.2 \mathrm{~Hz}, 1 \mathrm{H}, 5-\mathrm{H}_{\mathrm{a}}\right), 2.67\left(\mathrm{dt},{ }^{2} J_{5 \mathrm{~b}, 5 \mathrm{a}}=\right.$ $\left.13.9 \mathrm{~Hz},{ }^{3} J_{5 \mathrm{~b}, 6}=8.2 \mathrm{~Hz}, 1 \mathrm{H}, 5-\mathrm{H}_{\mathrm{b}}\right), 3.82(\mathrm{~m}, 2 \mathrm{H}, 18-\mathrm{H}), 6.20\left(\mathrm{dt},{ }^{3} J_{9,10}=15.4 \mathrm{~Hz},{ }^{3} J_{9,8}=6.9 \mathrm{~Hz}\right.$, $1 \mathrm{H}, 9-\mathrm{H}), 6.36\left(\mathrm{~d},{ }^{3} \mathrm{~J}_{10,9}=15.8 \mathrm{~Hz}, 1 \mathrm{H}, 10-\mathrm{H}\right), 7.10-7.20(\mathrm{~m}, 4 \mathrm{H}, 1-\mathrm{H}, 3-\mathrm{H}, 14-\mathrm{H}), 7.21-7.30$ (m, 4 H, 2-H, 13-H), 7.32 (m, 2 H, 12-H).

${ }^{13}$ C NMR (125 MHz, $\left.\mathrm{CDCl}_{3}\right): \delta=14.3$ (q, C.17), 21.4 (t, C-16), 26.0 (t, C-21), 26.1 (t, C-20), 26.6 (t, C-22), 27.9 (t, C-21 '), 28.8 (t, C-20`), 33.7 (t, C-5), 35.1 (t, C-6), 36.7 (t, C-8), 39.6 (d, C-7), 43.3 (d, C-19), 83.5 (d, C-18), 125.5 (d, C-1), 126.0 (d, C-12), 126.7 (d, C-14), 128.2 (d, C-2), 128.4 (d, C-3), 128.4 (d, C-13), 130.6 (d, C-9), 130.9 (d, C-10), 138.2 (s, C-11), 143.3 (s, C-4).

The signal of $\mathrm{C}-15$ could not be detected.

HRMS (CI) m/z calcd for $\mathrm{C}_{41} \mathrm{H}_{54} \mathrm{O}_{3} \mathrm{~B}[\mathrm{M}-\mathrm{H}]^{+}:$511.3742, found: 511.3770.

\section{Potassium $[(S, E)$-1,6-diphenylhex-5-en-3-yl] trifluorborate (28)}

$827 \mu \mathrm{l}$ (3.72 mmol, 7.0 eq.) $4.5 \mathrm{M}$ aqueous $\mathrm{KHF}_{2}$ solution were added dropwise to a vigorously stirred solution of $250 \mathrm{mg}(531 \mu \mathrm{mol}, 1.0 \mathrm{eq}$.) boronic ester $10 \mathrm{~g}$ in $4.7 \mathrm{ml}$ acetone at room temperature. After stirring for $2 \mathrm{~h}$, acetone was removed in vacuo and $\mathrm{MeCN}(5 \mathrm{ml})$ was added to the aqueous residue. The $\mathrm{MeCN} /$ water layer was washed twice with pentane $(15 \mathrm{ml})$ and concentrated in vacuo. The residue was dissolved in hot MeCN $(7.5 \mathrm{ml})$, filtered and washed twelve times with pentane $(10 \mathrm{ml})$. Concentration of the $\mathrm{MeCN}$ layer in vacuo gave the trifluoroborate salt $\mathbf{2 8}$ in $62 \%$ yield $(112 \mathrm{mg}, 327 \mu \mathrm{mol})$ as a colorless, amorphous solid, $\alpha_{D}^{20}=$ $-1.6(\mathrm{c}=0.5, \mathrm{MeOH})$. By concentration of the combined pentane layers in vacuo, $(S, S)$ DICHED was recovered in $82 \%$ yield $(98 \mathrm{mg}, 435 \mu \mathrm{mol})$ as a colorless solid, m.p. $142{ }^{\circ} \mathrm{C}$. Analytical data of $(S, S)$-DICHED were in accordance with those previously reported. ${ }^{13}$

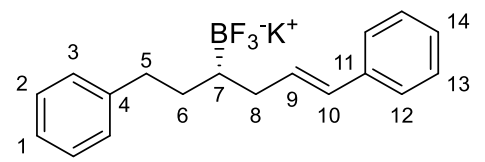

${ }^{\mathbf{1}} \mathbf{H}$ NMR $\left(400 \mathrm{MHz}, \mathrm{CD}_{3} \mathrm{CN}\right): \delta=0.40(\mathrm{~m}, 1 \mathrm{H}, 7-\mathrm{H}), 1.45\left(\mathrm{dddd},{ }^{2} J_{6 \mathrm{a}, 6 \mathrm{~b}}=13.3 \mathrm{~Hz},{ }^{3} J_{6 \mathrm{a}, 5 \mathrm{a}}=\right.$ $\left.9.3 \mathrm{~Hz},{ }^{3} J_{6 \mathrm{a}, 5 \mathrm{~b}} \approx{ }^{3} J_{6 \mathrm{a}, 7}=6.6 \mathrm{~Hz}, 1 \mathrm{H}, 6-\mathrm{H}_{\mathrm{a}}\right), 1.58\left(\mathrm{dddd},{ }^{3} J_{6 \mathrm{~b}, 6 \mathrm{a}}=13.6 \mathrm{~Hz},{ }^{3} J_{6 \mathrm{~b}, 5 \mathrm{~b}}=9.3 \mathrm{~Hz},{ }^{3} J_{6 \mathrm{~b}, 5 \mathrm{a}} \approx\right.$ $\left.{ }^{3} J_{6 \mathrm{~b}, 7}=6.8 \mathrm{~Hz}, 1 \mathrm{H}, 6-\mathrm{H}_{\mathrm{b}}\right), 2.08\left(\mathrm{ddd},{ }^{2} J_{8 \mathrm{a}, 8 \mathrm{~b}}=14.0 \mathrm{~Hz},{ }^{3} J_{8 \mathrm{a}, 7} \approx{ }^{3} J_{8 \mathrm{a}, 9}=8.2 \mathrm{~Hz}, 1 \mathrm{H}, 8-\mathrm{H}_{\mathrm{a}}\right), 2.30$ $\left(\mathrm{ddd},{ }^{2} J_{8 \mathrm{~b}, 8 \mathrm{a}}=14.2 \mathrm{~Hz},{ }^{3} J_{8 \mathrm{~b}, 7} \approx{ }^{3} J_{8 \mathrm{~b}, 9}=5.3 \mathrm{~Hz}, 1 \mathrm{H}, 8-\mathrm{H}_{\mathrm{b}}\right), 2.64(\mathrm{~m}, 2 \mathrm{H}, 5-\mathrm{H}), 6.31\left(\mathrm{~d},{ }^{3} J_{10,9}=\right.$ $15.9 \mathrm{~Hz}, 1 \mathrm{H}, 10-\mathrm{H}), 6.43\left(\mathrm{dt},{ }^{3} J_{9,10}=15.8 \mathrm{~Hz},{ }^{3} J_{9,8}=7.1 \mathrm{~Hz}, 1 \mathrm{H}, 9-\mathrm{H}\right), 7.09(\mathrm{~m}, 1 \mathrm{H}, 1-\mathrm{H})$, 7.06-7.12 (m, 5 H, 3-H, 13-H, 14-H), 7.27 (m, 2 H, 2-H), 7.34 (m, 2 H, 12-H). 
${ }^{13}$ C NMR (100 MHz, CD 3 CN): $\delta=29.1$ (bs, C-7), 34.1 (t, C-6), 35.7 (t, C-8), 36.2 (t, C-5), 126.0 (d, C-1), 126.7 (d, C-12), 127.3 (d, C-14), 129.1 (d, C-13), 129.2 (d, C-10), 129.5 (d, C-2), 129.5 (d, C-3), 136.0 (d, C-9), 139.7 (s, C-11), 146.1 (s, C-4).

${ }^{11}$ B-NMR (128 MHz, $\left.\mathrm{CD}_{3} \mathrm{CN}\right): \delta=5.3(\mathrm{bs})$.

HRMS (CI) m/z calcd for $\mathrm{C}_{18} \mathrm{H}_{20} \mathrm{BF}_{2}[\mathrm{M}+\mathrm{H}-\mathrm{KF}]^{+}: 285.1621$, found: 285.1641 .

\section{(S)-4-[(4S,5S)-4,5-Dicyclohexyl-1,3,2-dioxaborolan-2-yl]-6-phenylhexan-2-one (29a)}

$82 \mathrm{mg}(201 \mu \mathrm{mol})$ boronic ester 10a were subjected to ozonolysis according to GP 5 over 3 min. After reductive workup with $63 \mathrm{mg}(241 \mu \mathrm{mol})$ triphenylphosphine, the crude product was purified by flash chromatography (pentane, ethyl acetate 9:1) to give 29a in $76 \%$ yield (63 mg, $153 \mu \mathrm{mol})$ as a colorless oil which was sufficiently pure to be used in the next step. An analytically pure sample of 29a was obtained by reversed-phase flash chromatography $\left(\mathrm{H}_{2} \mathrm{O}\right.$, $70 \% \mathrm{MeCN} \rightarrow 100 \% \mathrm{MeCN}$ ) to remove trace impurities of excess triphenylphosphine; $\mathrm{R}_{\mathrm{f}}=$ 0.29 (pentane, ethyl acetate 9:1); $\alpha_{D}^{20}=-43.6\left(\mathrm{c}=0.5, \mathrm{CHCl}_{3}\right)$.

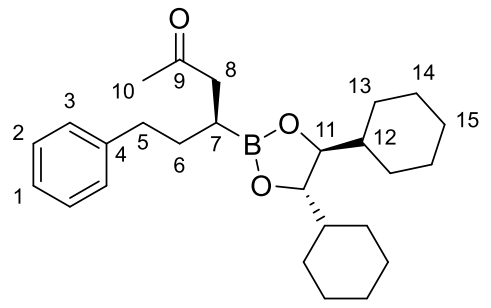

${ }^{1} \mathbf{H}$ NMR $\left(400 \mathrm{MHz}, \mathrm{CDCl}_{3}\right): \delta=0.96(\mathrm{~m}, 2 \mathrm{H}, 14-\mathrm{H}), 1.05(\mathrm{~m}, 2 \mathrm{H}, 13-\mathrm{H}), 1.09-1.43(\mathrm{~m}, 9 \mathrm{H}$, 7-H, 12-H, 13-H', 14-H'), 1.52-1.65 (m, 3 H, 6-Ha, 13-H'”), 1.68 (m, 2 H, 18-H), 1.71-1.85 (m, $7 \mathrm{H}, 6-\mathrm{H}_{\mathrm{b}}, 13-\mathrm{H}^{\prime}$ ',, $14-\mathrm{H}^{\prime}$,, $\left.15-\mathrm{H}^{\prime}\right), 2.11$ (s, $\left.3 \mathrm{H}, 10-\mathrm{H}\right), 2.54-2.72$ (m, $\left.4 \mathrm{H}, 5-\mathrm{H}, 8-\mathrm{H}\right), 3.83$ (m, 2 H, 11-H), 7.13-7.20 (m, 3 H, 1-H, 3-H), 7.26 (m, 2 H, 2-H).

${ }^{13} \mathrm{C} \mathrm{NMR}\left(100 \mathrm{MHz}, \mathrm{CDCl}_{3}\right): \delta=25.9$ (t, C-14), 26.0 (t, C-13), 26.5 (t, C-15), 27.7 (t, C-14'), 28.5 (t, C-13'), 29.7 (q, C-10), 33.0 (t, C-6), 35.4 (t, C-5), 42.9 (d, C-12), 45.8 (t, C-8), 83.5 (d, C-11), 125.6 (d, C-1), 128.3 (d, C-2), 128.4 (d, C-3), 142.7 (s, C-4), 209.0 (s, C-9).

HRMS (CI) m/z calcd for $\mathrm{C}_{26} \mathrm{H}_{40} \mathrm{O}_{3} \mathrm{~B}[\mathrm{M}+\mathrm{H}]^{+}:$:11.3065, found: 411.3078 .

\section{(S)-3-[(4S,5S)-4,5-Dicyclohexyl-1,3,2-dioxaborolan-2-yl]-5-phenylpentanal (29b)}

$100 \mathrm{mg}(213 \mu \mathrm{mol})$ boronic ester $\mathbf{1 0 g}$ were subjected to ozonolysis according to GP 5 over $1.5 \mathrm{~min}$. After reductive workup with $67 \mathrm{mg}(255 \mu \mathrm{mol})$ triphenylphosphine, the crude product was purified by flash chromatography (pentane, ethyl acetate 95:5) to give 29b in $83 \%$ yield (70 $\mathrm{mg}, 177 \mu \mathrm{mol}$ ) as a colorless oil which was sufficiently pure to be used in the next step. An analytically pure sample of 29b was obtained by reversed-phase flash chromatography $\left(\mathrm{H}_{2} \mathrm{O}\right.$, $70 \% \mathrm{MeCN} \rightarrow 100 \% \mathrm{MeCN}$ ) to remove trace impurities of excess triphenylphosphine; $\mathrm{R}_{\mathrm{f}}=$ 0.22 (pentane, ethyl acetate 95:5); $\alpha_{D}^{20}=-44.8\left(\mathrm{c}=0.5, \mathrm{CHCl}_{3}\right)$.

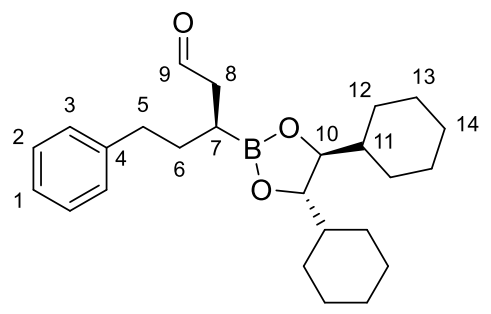

${ }^{1} \mathbf{H}$ NMR (400 MHz, $\left.\mathrm{CDCl}_{3}\right): \delta=0.97(\mathrm{~m}, 2 \mathrm{H}, 13-\mathrm{H}), 1.06$ (m, $\left.2 \mathrm{H}, 12-\mathrm{H}\right), 1.11-1.29(\mathrm{~m}, 6 \mathrm{H}$, 12-H', 13-H'), 1.34 (m, $2 \mathrm{H}, 11-\mathrm{H}), 1.47$ (dddd, ${ }^{3} J_{7,6 \mathrm{~b}} \approx{ }^{3} J_{7,8 \mathrm{a}}=7.9 \mathrm{~Hz},{ }^{3} J_{7,6 \mathrm{a}} \approx{ }^{3} J_{7,8 \mathrm{~b}}=6.4 \mathrm{~Hz}$, 
$1 \mathrm{H}, 7-\mathrm{H}), 1.58-1.72\left(\mathrm{~m}, 5 \mathrm{H}, 6-\mathrm{H}_{\mathrm{a}}, 12-\mathrm{H}\right.$ ', 14-H), 1.72-1.89 (m, $7 \mathrm{H}, 6-\mathrm{H}_{\mathrm{b}}, 12-\mathrm{H}^{\prime \prime}$, , 13-H', 14-H'), $2.56\left(\mathrm{ddd},{ }^{2} J_{8 \mathrm{a}, 8 \mathrm{~b}}=17.9 \mathrm{~Hz},{ }^{3} J_{8 \mathrm{a}, 7}=6.2 \mathrm{~Hz},{ }^{3} J_{8 \mathrm{a}, 9}=1.3 \mathrm{~Hz}, 1 \mathrm{H}, 8-\mathrm{H}_{\mathrm{a}}\right), 2.59-2.74(\mathrm{~m}$, $\left.3 \mathrm{H}, 5-\mathrm{H}, 8-\mathrm{H}_{\mathrm{b}}\right), 3.86(\mathrm{~m}, 2 \mathrm{H}, 10-\mathrm{H}), 7.14-7.21(\mathrm{~m}, 3 \mathrm{H}, 1-\mathrm{H}, 3-\mathrm{H}), 7.27$ (m, $\left.2 \mathrm{H}, 2-\mathrm{H}\right), 9.76$ (t, $\left.{ }^{3} J_{9,8}=1.3 \mathrm{~Hz}, 1 \mathrm{H}, 9-\mathrm{H}\right)$.

${ }^{13} \mathrm{C}$ NMR (100 MHz, CDCl 3 ): $\delta=16.8$ (bs, C-7), 25.9 (t, C-13), 16.0 (t, C-12), 26.4 (t, C-14), 27.5 (t, C-13'), 28.5 (t, C-12'), 32.9 (t, C-6), 35.2 (t, C-5), 43.0 (d, C-11), 45.8 (t, C-8), 83.6 (d, C-10), 125.7 (d, C-1), 128.3 (d, C-2), 128.4 (d, C-3), 142.4 (s, C-4), 202.8 (d, C-9).

HRMS (CI) m/z calcd for $\mathrm{C}_{25} \mathrm{H}_{38} \mathrm{O}_{3} \mathrm{~B}[\mathrm{M}+\mathrm{H}]^{+}:$397.2909, found: 397.2887.

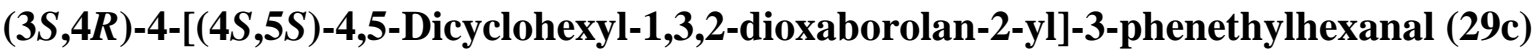

$166 \mathrm{mg}(324 \mu \mathrm{mol})$ boronic ester 27 were subjected to ozonolysis according to GP 5 over $1.5 \mathrm{~min}$. After reductive workup with $102 \mathrm{mg}(389 \mu \mathrm{mol})$ triphenylphosphine, the crude product was purified by flash chromatography (pentane, ethyl acetate 95:5) to give 29c in $76 \%$ yield $(107 \mathrm{mg}, 245 \mu \mathrm{mol})$ as a colorless oil which was sufficiently pure to be used in the next step. An analytically pure sample of 29c was obtained by reversed-phase flash chromatography $\left(\mathrm{H}_{2} \mathrm{O}, 70 \% \mathrm{MeCN} \rightarrow 100 \% \mathrm{MeCN}\right)$ to remove trace impurities of excess triphenylphosphine; $\mathrm{R}_{\mathrm{f}}=0.18$ (pentane, ethyl acetate 95:5); $\alpha_{D}^{20}=-40.4\left(\mathrm{c}=0.5, \mathrm{CHCl}_{3}\right)$.

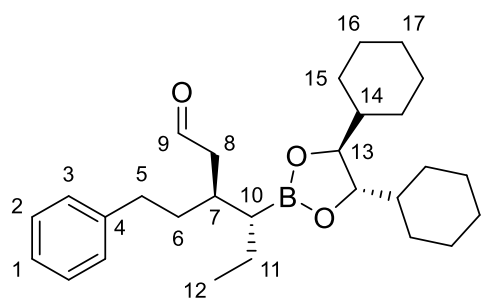

${ }^{1} \mathbf{H}$ NMR $\left(400 \mathrm{MHz}, \mathrm{CDCl}_{3}\right): \delta=0.89-1.00\left(\mathrm{~m},{ }^{3} \mathrm{~J}_{12,11}=7.3 \mathrm{~Hz}, 5 \mathrm{H}, 12-\mathrm{H}, 16-\mathrm{H}\right), 1.06(\mathrm{~m}$, $2 \mathrm{H}, 15-\mathrm{H}), 1.11-1.41$ (m, $\left.10 \mathrm{H}, 10-\mathrm{H}, 11-\mathrm{H}_{\mathrm{a}}, 14-\mathrm{H}, 15-\mathrm{H}^{\prime}, 16-\mathrm{H}^{\prime}\right), 1.52$ (m, $\left.1 \mathrm{H}, 11-\mathrm{H}_{\mathrm{b}}\right), 1.56-$ 1.73 (m, 6 H, 6-H, 15-H', 17-H), 1.73-1.84 (m, 6 H, 15-H', , 16-H', 17-H'), 2.24 (m, $1 \mathrm{H}$, 7-H), $2.40\left(\mathrm{ddd},{ }^{2} J_{8 \mathrm{a}, 8 \mathrm{~b}}=16.3 \mathrm{~Hz},{ }^{3} J_{8 \mathrm{a}, 7}=8.2 \mathrm{~Hz},{ }^{3} J_{8 \mathrm{a}, 9}=2.8 \mathrm{~Hz}, 1 \mathrm{H}, 8-\mathrm{H}_{\mathrm{a}}\right), 2.50-2.60(\mathrm{~m}, 2 \mathrm{H}$, $\left.5-\mathrm{H}_{\mathrm{a}}, 8-\mathrm{H}_{\mathrm{b}}\right), 2.66\left(\mathrm{ddd},{ }^{2} J_{5 \mathrm{~b}, 5 \mathrm{a}}=13.4 \mathrm{~Hz},{ }^{3} J_{5 \mathrm{~b}, 6 \mathrm{~b}}=10.4 \mathrm{~Hz},{ }^{3} J_{5 \mathrm{~b}, 6 \mathrm{a}}=5.7 \mathrm{~Hz}, 1 \mathrm{H}, 5-\mathrm{H}_{\mathrm{b}}\right), 3.83(\mathrm{~m}$, $2 \mathrm{H}, 13-\mathrm{H}), 7.13-7.20(\mathrm{~m}, 3 \mathrm{H}, 1-\mathrm{H}, 3-\mathrm{H}), 7.26(\mathrm{~m}, 2 \mathrm{H}, 2-\mathrm{H}), 9.75\left(\mathrm{dd},{ }^{3} J_{9,8 \mathrm{a}}=2.7 \mathrm{~Hz},{ }^{3} J_{9,8 \mathrm{~b}}=\right.$ $1.8 \mathrm{~Hz}, 1 \mathrm{H}, 9-\mathrm{H})$.

${ }^{13} \mathrm{C}$ NMR $\left(100 \mathrm{MHz}, \mathrm{CDCl}_{3}\right): \delta=14.1$ (q, C-12), 22.0 (t, C-11), 22.9 (t, C-16), 26.0 (t, C-15), 26.4 (t, C-17), 27.7 (t, C-16'), 28.6 (t, C-15'), 29.1 (bs, C-10), 33.6 (t, C-5), 33.8 (d, C-7), 36.6 (t, C-6), 43.1 (d, C-14), 47.5 (t, C-8), 83.5 (d, C-13), 125.7 (d, C-1), 128.3 (d, C-2, C-3), 142.4 (s, C-4), 203.6 (d, C-9).

HRMS (CI) m/z calcd for $\mathrm{C}_{28} \mathrm{H}_{42} \mathrm{O}_{3} \mathrm{~B}[\mathrm{M}-\mathrm{H}]^{+}$: 437.3222, found: 437.3210 .

\section{(S)-4-Hydroxy-6-phenylhexan-2-one (30a)}

$45 \mathrm{mg}(110 \mu \mathrm{mol})$ boronic ester 29a, $51 \mu \mathrm{l}(33 \%$ in water, $\rho=1.11 \mathrm{~g} / \mathrm{ml}, 548 \mu \mathrm{mol})$ hydrogen peroxide and $22 \mathrm{mg}(548 \mu \mathrm{mol})$ sodium hydroxide were reacted according to GP 2 . After $2.5 \mathrm{~h}$, the reaction was worked up and the crude product was treated with $8 \mathrm{mg}(132 \mu \mathrm{mol})$ methylboronic acid to separate $(S, S)$-DICHED. Purification by flash chromatography $\left(\mathrm{Al}_{2} \mathrm{O}_{3}\right.$; pentane, diethyl ether 1:3) gave 30a in $85 \%$ yield $(18 \mathrm{mg}, 94 \mu \mathrm{mol})$ as a colorless oil, $\mathrm{R}_{\mathrm{f}}=0.27$ $\left(\mathrm{Al}_{2} \mathrm{O}_{3}\right.$; pentane, diethyl ether $\left.1: 3\right) ; \alpha_{D}^{20}=+12.1\left(\mathrm{c}=0.5, \mathrm{CHCl}_{3}\right)$.

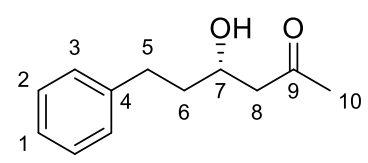


${ }^{1}$ H NMR $\left(400 \mathrm{MHz}, \mathrm{CDCl}_{3}\right): \delta=1.68\left(\mathrm{~m}, 1 \mathrm{H}, 6-\mathrm{H}_{\mathrm{a}}\right), 1.82\left(\mathrm{~m}, 1 \mathrm{H}, 6-\mathrm{H}_{\mathrm{b}}\right), 2.16(\mathrm{~s}, 3 \mathrm{H}, 10-\mathrm{H})$, $2.56\left(\mathrm{dd},{ }^{2} J_{8 \mathrm{a}, 8 \mathrm{~b}}=17.7 \mathrm{~Hz},{ }^{3} J_{8 \mathrm{a}, 7}=8.4 \mathrm{~Hz}, 1 \mathrm{H}, 8-\mathrm{H}_{\mathrm{a}}\right), 2.63\left(\mathrm{dd},{ }^{2} J_{8 \mathrm{~b}, 8 \mathrm{a}}=17.6 \mathrm{~Hz},{ }^{3} J_{8 \mathrm{~b}, 7}=3.5 \mathrm{~Hz}\right.$, $1 \mathrm{H}, 8-\mathrm{H}_{\mathrm{b}}$ ), $2.68\left(\mathrm{ddd},{ }^{2} J_{5 \mathrm{a}, 5 \mathrm{~b}}=13.8 \mathrm{~Hz},{ }^{3} J_{5 \mathrm{a}, 6 \mathrm{a}}=9.5 \mathrm{~Hz},{ }^{3} J_{5 \mathrm{a}, 6 \mathrm{~b}}=7.2 \mathrm{~Hz}, 1 \mathrm{H}, 5-\mathrm{H}_{\mathrm{a}}\right), 2.81$ (ddd, $\left.{ }^{2} J_{5 \mathrm{~b}, 5 \mathrm{a}}=14.2 \mathrm{~Hz},{ }^{3} J_{5 \mathrm{~b}, 6 \mathrm{~b}}=9.7 \mathrm{~Hz},{ }^{3} J_{5 \mathrm{~b}, 6 \mathrm{a}}=5.4 \mathrm{~Hz}, 1 \mathrm{H}, 5-\mathrm{H}_{\mathrm{b}}\right), 3.09\left(\mathrm{~d},{ }^{3} J_{\mathrm{OH}, 7}=3.3 \mathrm{~Hz}, 1 \mathrm{H}, \mathrm{OH}\right)$, 4.05 (ddddd, $\left.{ }^{3} J_{7,6 \mathrm{a}} \approx{ }^{3} J_{7,8 \mathrm{a}}=8.2 \mathrm{~Hz},{ }^{3} J_{7,6 \mathrm{~b}} \approx{ }^{3} J_{7,8 \mathrm{~b}} \approx{ }^{3} J_{7, \mathrm{OH}}=3.9 \mathrm{~Hz}, 1 \mathrm{H}, 7-\mathrm{H}\right), 7.14-7.23(\mathrm{~m}$, $3 \mathrm{H}, 1-\mathrm{H}, 3-\mathrm{H}), 7.28$ (m, $2 \mathrm{H}, 2-\mathrm{H})$.

${ }^{13}$ C NMR (100 MHz, $\mathrm{CDCl}_{3}$ ): $\delta=30.7$ (q, C-10), 31.7 (t, C-5), 37.9 (t, C-6), 49.9 (t, C-8), 66.7 (d, C-7), 125.8 (d, C-1), 128.4 (d, C-2, C-3), 141.8 (s, C-4), 209.9 (s, C-9).

HRMS (CI) m/z calcd for $\mathrm{C}_{12} \mathrm{H}_{17} \mathrm{O}_{2}[\mathrm{M}+\mathrm{H}]^{+}:$193.1223, found: 193.1224 .

\section{(4S,5R)-5-Ethyl-4-phenethyltetrahydrofuran-2-ol (30b)}

$84 \mathrm{mg}(192 \mu \mathrm{mol})$ boronic ester 29c, $89 \mu \mathrm{l}(33 \%$ in water, $\rho=1.11 \mathrm{~g} / \mathrm{ml}, 958 \mu \mathrm{mol})$ hydrogen peroxide and $38 \mathrm{mg}(958 \mu \mathrm{mol})$ sodium hydroxide were reacted according to GP 2 . After $1 \mathrm{~h}$, the reaction was worked up and the crude product was treated with $14 \mathrm{mg}(230 \mu \mathrm{mol})$ methylboronic acid to separate $(S, S)$-DICHED. Purification by flash chromatography (pentane, diethyl ether 1:1) gave 30b (anomeric ratio $=1: 1$ according to $\left.{ }^{1} \mathrm{H} N M R\right)$ in $78 \%$ yield $(33 \mathrm{mg}$, $150 \mu \mathrm{mol})$ as a colorless oil, $\mathrm{R}_{\mathrm{f}}=0.28$ (pentane, diethyl ether $\left.1: 1\right) ; \alpha_{D}^{20}=+48.6\left(\mathrm{c}=1.0, \mathrm{CHCl}_{3}\right)$.

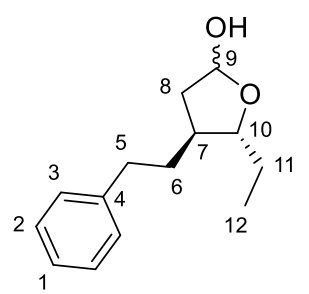

anomer 1:

${ }^{1} \mathbf{H}$ NMR $\left(500 \mathrm{MHz}, \mathrm{CDCl}_{3}\right): \delta=0.94\left(\mathrm{t},{ }^{3} J_{12,11}=7.4 \mathrm{~Hz}, 3 \mathrm{H}, 12-\mathrm{H}\right), 1.44\left(\mathrm{ddq},{ }^{2} J_{11 \mathrm{a}, 11 \mathrm{~b}}=\right.$ $\left.14.4 \mathrm{~Hz},{ }^{3} J_{11 \mathrm{a}, 10} \approx^{3} J_{11 \mathrm{a}, 12}=7.3 \mathrm{~Hz}, 1 \mathrm{H}, 11-\mathrm{H}_{\mathrm{a}}\right), 1.57-1.64\left(\mathrm{~m}, 2 \mathrm{H}, 8-\mathrm{H}_{\mathrm{a}}, 11-\mathrm{H}_{\mathrm{b}}\right), 1.68(\mathrm{~m}, 1 \mathrm{H}$, $\left.6-\mathrm{H}_{\mathrm{a}}\right), 1.76(\mathrm{~m}, 1 \mathrm{H}, 7-\mathrm{H}), 1.84\left(\mathrm{~m}, 1 \mathrm{H}, 6-\mathrm{H}_{\mathrm{b}}\right), 2.35\left(\mathrm{ddd},{ }^{2} J_{8 \mathrm{~b}, 8 \mathrm{a}}=13.5 \mathrm{~Hz}, 8.5 \mathrm{~Hz},{ }^{3} J_{8 \mathrm{~b}, 9}=\right.$ $\left.5.4 \mathrm{~Hz}, 1 \mathrm{H}, 8-\mathrm{H}_{\mathrm{b}}\right), 2.57\left(\mathrm{ddd},{ }^{2} J_{5 \mathrm{a}, 5 \mathrm{~b}}=13.9 \mathrm{~Hz},{ }^{3} J_{5 \mathrm{a}, 6 \mathrm{a}}=9.9 \mathrm{~Hz},{ }^{3} J_{5 \mathrm{a}, 6 \mathrm{~b}}=6.7 \mathrm{~Hz}, 1 \mathrm{H}, 5-\mathrm{H}_{\mathrm{a}}\right), 2.68$ $\left(\mathrm{ddd},{ }^{2} J_{5 \mathrm{~b}, 5 \mathrm{a}}=14.4 \mathrm{~Hz},{ }^{3} J_{5 \mathrm{~b}, 6 \mathrm{~b}}=10.3 \mathrm{~Hz},{ }^{3} J_{5 \mathrm{~b}, 6 \mathrm{a}}=5.4 \mathrm{~Hz}, 1 \mathrm{H}, 5-\mathrm{H}_{\mathrm{b}}\right), 3.70(\mathrm{bs}, 1 \mathrm{H}, \mathrm{OH}), 3.78$ $\left(\mathrm{ddd},{ }^{3} J_{10,11 \mathrm{a}} \approx{ }^{3} J_{10,7}=7.7 \mathrm{~Hz},{ }^{3} J_{10,11 \mathrm{a}}=4.0 \mathrm{~Hz}, 1 \mathrm{H}, 10-\mathrm{H}\right), 5.53\left(\mathrm{ddd},{ }^{3} J_{9,8 \mathrm{~b}}=5.4 \mathrm{~Hz},{ }^{3} J_{9,8 \mathrm{a}} \approx\right.$ $\left.{ }^{3} J_{9, \mathrm{OH}}=3.1 \mathrm{~Hz}, 1 \mathrm{H}, 9-\mathrm{H}\right), 7.12-7.21(\mathrm{~m}, 3 \mathrm{H}, 1-\mathrm{H}, 3-\mathrm{H}), 7.27(\mathrm{~m}, 2 \mathrm{H}, 2-\mathrm{H})$.

${ }^{13}$ C NMR (125 MHz, $\left.\mathrm{CDCl}_{3}\right): \delta=10.4$ (q, C-12), 27.1 (t, C-11), 34.6 (t, C-5), 34.9 (t, C-6), 40.0 (t, C-8), 43.0 (d, C-7), 84.5 (d, C-10), 98.0 (d, C-9), 125.8 (d, C-1), 128.2 (d, C-3), 128.4 (d, C-2), 141.9 (s, C-4).

anomer 2:

${ }^{1} \mathbf{H}$ NMR $\left(500 \mathrm{MHz}, \mathrm{CDCl}_{3}\right): \delta=0.97\left(\mathrm{t},{ }^{3} J_{12,11}=7.4 \mathrm{~Hz}, 3 \mathrm{H}, 12-\mathrm{H}\right), 1.51\left(\mathrm{~m}, 1 \mathrm{H}, 6-\mathrm{H}_{\mathrm{a}}\right), 1.57$ $\left(\mathrm{ddq},{ }^{2} J_{11 \mathrm{a}, 11 \mathrm{~b}}=13.9 \mathrm{~Hz},{ }^{3} J_{11 \mathrm{a}, 10} \approx{ }^{3} J_{11 \mathrm{a}, 12}=7.6 \mathrm{~Hz}, 1 \mathrm{H}, 11-\mathrm{H}_{\mathrm{a}}\right), 1.62-1.72\left(\mathrm{~m}, 2 \mathrm{H}, 8-\mathrm{H}_{\mathrm{a}}, 11-\mathrm{H}_{\mathrm{b}}\right)$, $1.83\left(\mathrm{~m}, 1 \mathrm{H}, 6-\mathrm{H}_{\mathrm{b}}\right), 2.08-2.18\left(\mathrm{~m}, 2 \mathrm{H}, 7-\mathrm{H}, 8-\mathrm{H}_{\mathrm{b}}\right), 2.59\left(\mathrm{ddd},{ }^{2} J_{5 \mathrm{a}, 5 \mathrm{~b}}=13.5 \mathrm{~Hz},{ }^{3} J_{5 \mathrm{a}, 6 \mathrm{a}}=10.3 \mathrm{~Hz}\right.$, $\left.{ }^{3} J_{5 \mathrm{a}, 6 \mathrm{~b}}=6.3 \mathrm{~Hz}, 1 \mathrm{H}, 5-\mathrm{H}_{\mathrm{a}}\right), 2.66\left(\mathrm{ddd},{ }^{2} J_{5 \mathrm{~b}, 5 \mathrm{a}}=13.9 \mathrm{~Hz},{ }^{3} J_{5 \mathrm{~b}, 6 \mathrm{~b}}=10.3 \mathrm{~Hz},{ }^{3} J_{5 \mathrm{~b}, 6 \mathrm{a}}=5.4 \mathrm{~Hz}, 1 \mathrm{H}\right.$, $\left.5-\mathrm{H}_{\mathrm{b}}\right), 3.50(\mathrm{bs}, 1 \mathrm{H}, \mathrm{OH}), 3.56\left(\mathrm{ddd},{ }^{3} J_{10,11 \mathrm{a}} \approx{ }^{3} J_{10,7}=7.7 \mathrm{~Hz},{ }^{3} J_{10,11 \mathrm{a}}=4.3 \mathrm{~Hz}, 1 \mathrm{H}, 10-\mathrm{H}\right), 5.47$ $\left(\mathrm{dd},{ }^{3} J_{9,8 \mathrm{~b}}=4.9 \mathrm{~Hz},{ }^{3} J_{9,8 \mathrm{a}}=3.1 \mathrm{~Hz}, 1 \mathrm{H}, 9-\mathrm{H}\right), 7.12-7.21(\mathrm{~m}, 3 \mathrm{H}, 1-\mathrm{H}, 3-\mathrm{H}), 7.27(\mathrm{~m}, 2 \mathrm{H}, 2-\mathrm{H})$.

${ }^{13}$ C NMR (125 MHz, $\left.\mathrm{CDCl}_{3}\right): \delta=10.5$ (t, C-12), 29.0 (t, C-11), 34.7 (t, C-5), 34.9 (t, C-6), 40.5 (t, C-9), 41.4 (d, C-7), 87.1 (d, C-10), 97.8 (d, C-9), 125.8 (d, C-1), 128.2 (d, C-3), 128.3 (d, C-2), 141.9 (s, C-4).

HRMS (CI) $\mathrm{m} / \mathrm{z}$ calcd for $\mathrm{C}_{14} \mathrm{H}_{21} \mathrm{O}_{2}[\mathrm{M}+\mathrm{H}]^{+}:$221.1536, found: 221.1538 . 


\section{Copies of the NMR spectra, HPLC and GC chromatograms}

$(4 S, 5 S)-4,5-D i c y c l o h e x y l-2-[(S)-1-p h e n y l h e x-5-e n-3-y l]-1,3,2$-dioxaborolane $(2$, crude product)

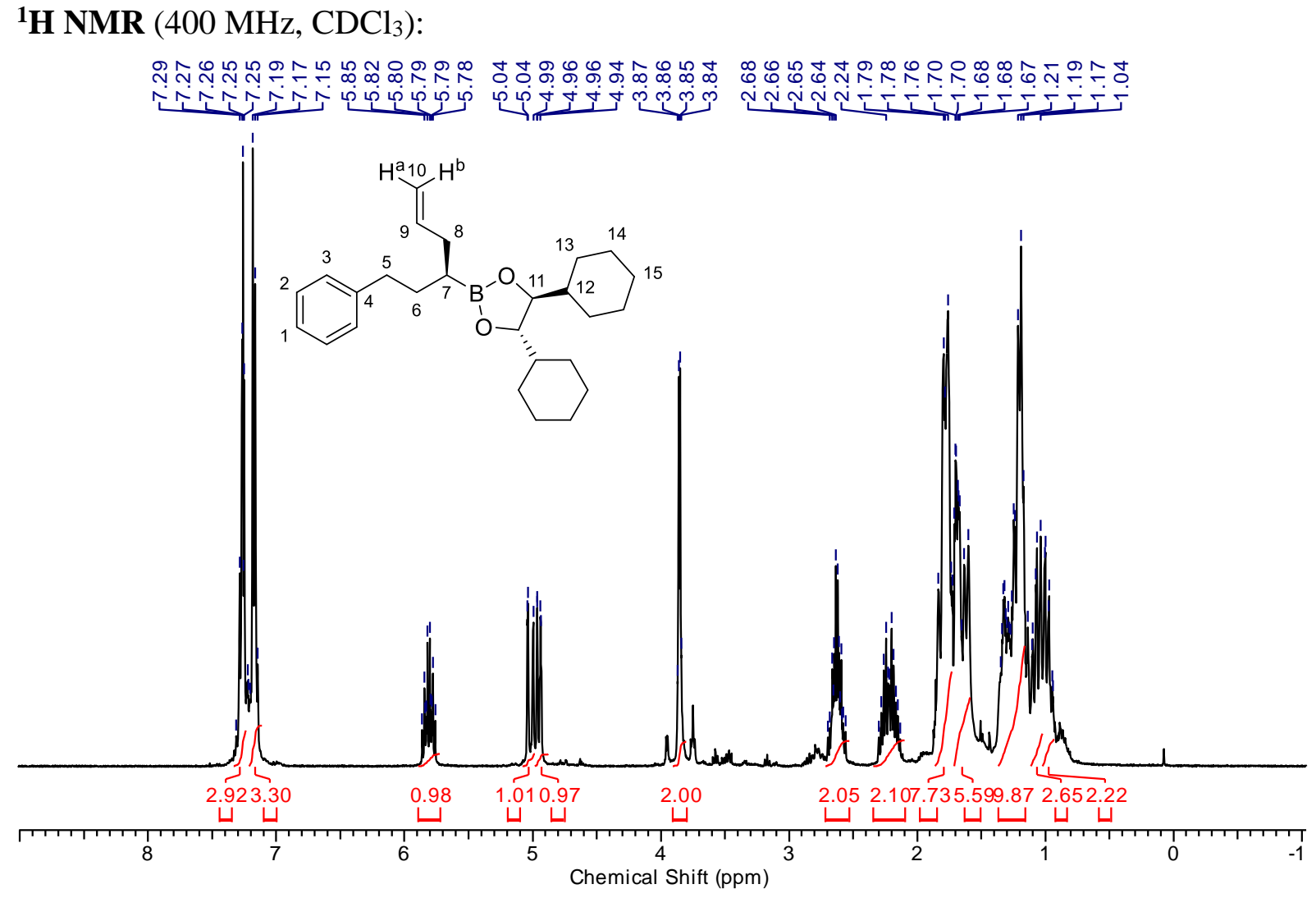

${ }^{13} \mathrm{C}$ NMR (100 MHz, $\left.\mathrm{CDCl}_{3}\right)$ :

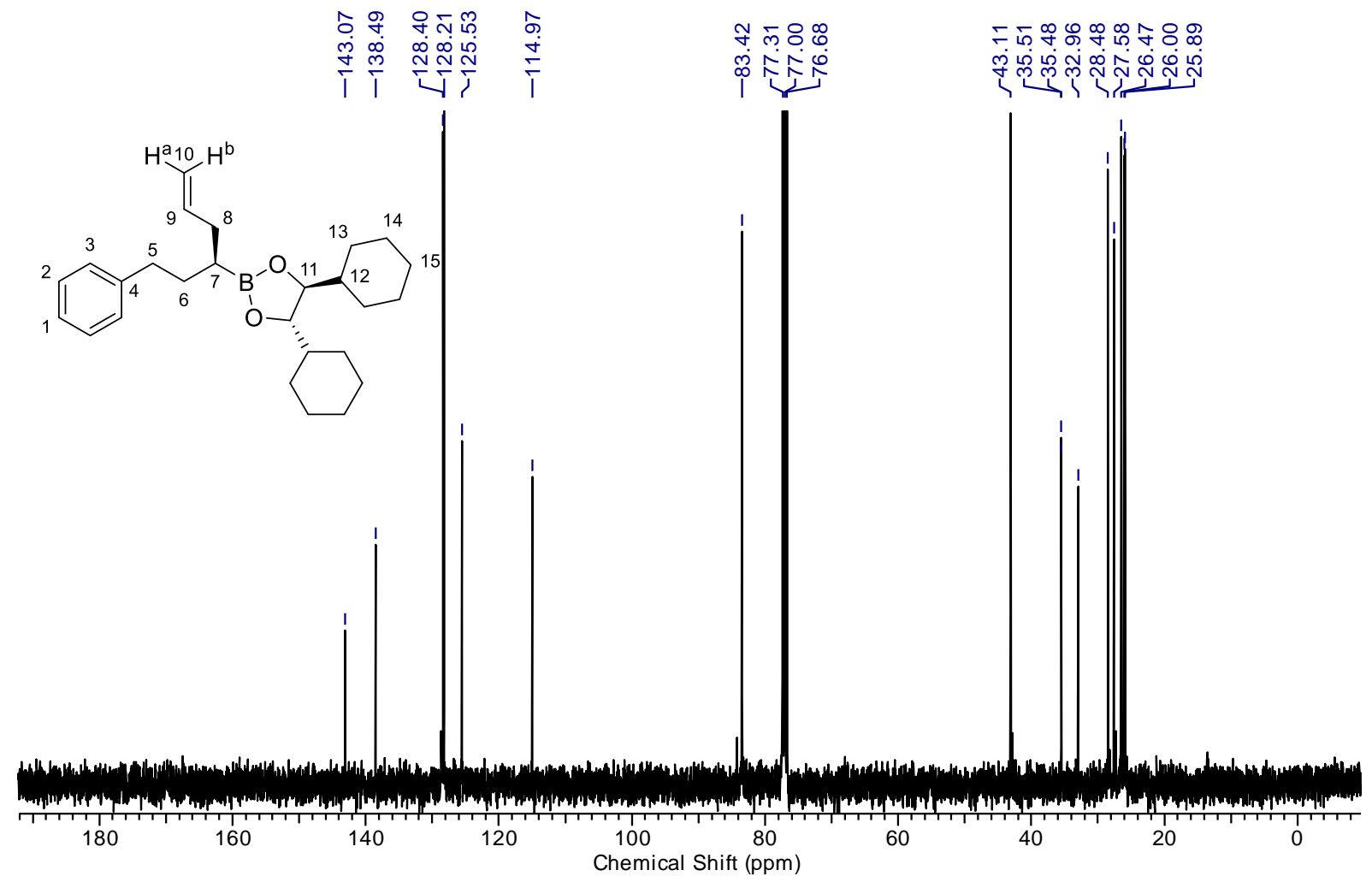




\section{(S)-1-Phenylhex-5-en-3-ol (3)}

\section{${ }^{1} \mathbf{H}$ NMR (400 MHz, $\left.\mathrm{CDCl}_{3}\right)$ :}

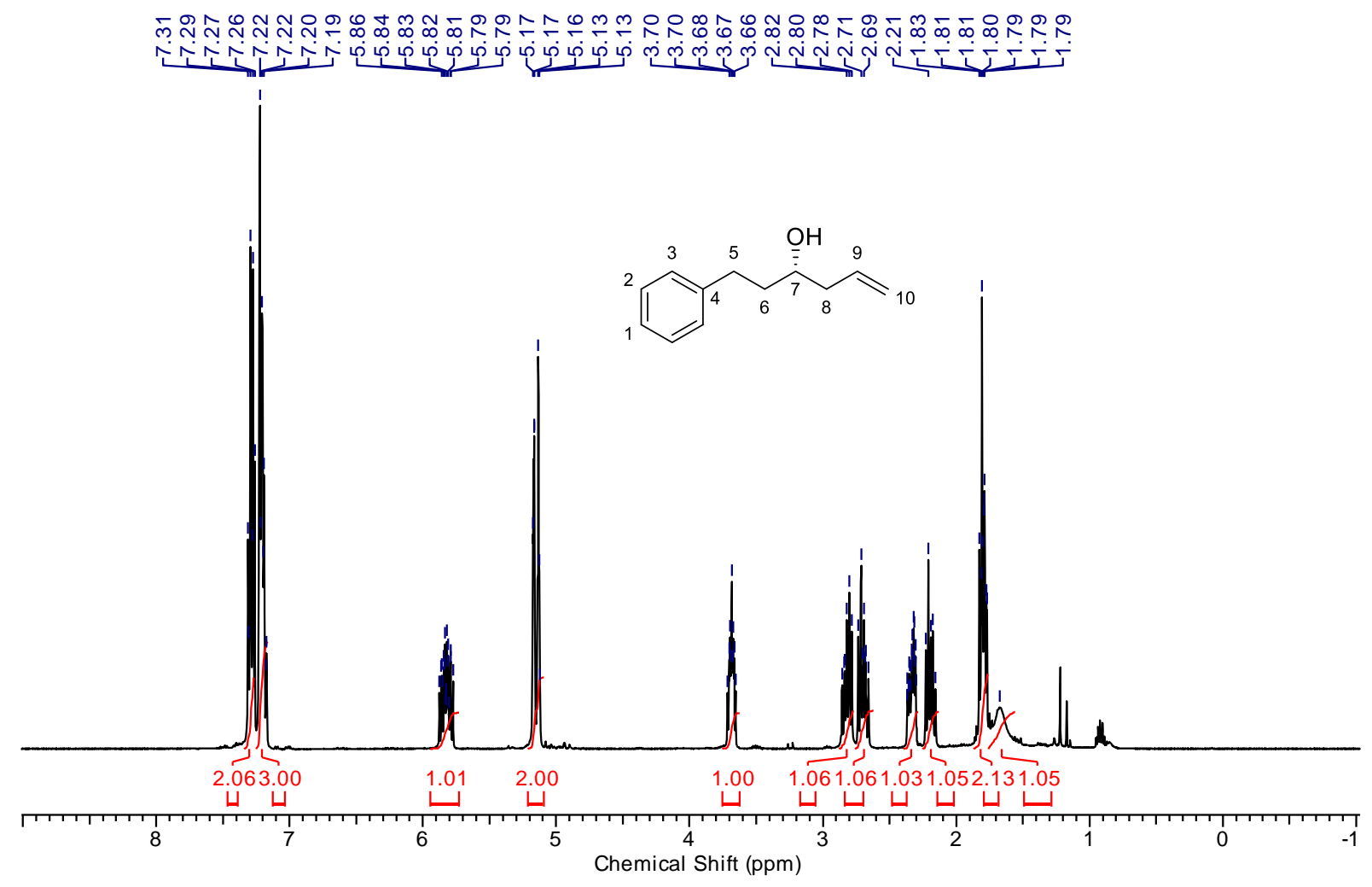

${ }^{13}$ C NMR $\left(100 \mathrm{MHz}, \mathrm{CDCl}_{3}\right)$ :

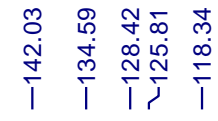

뜌:

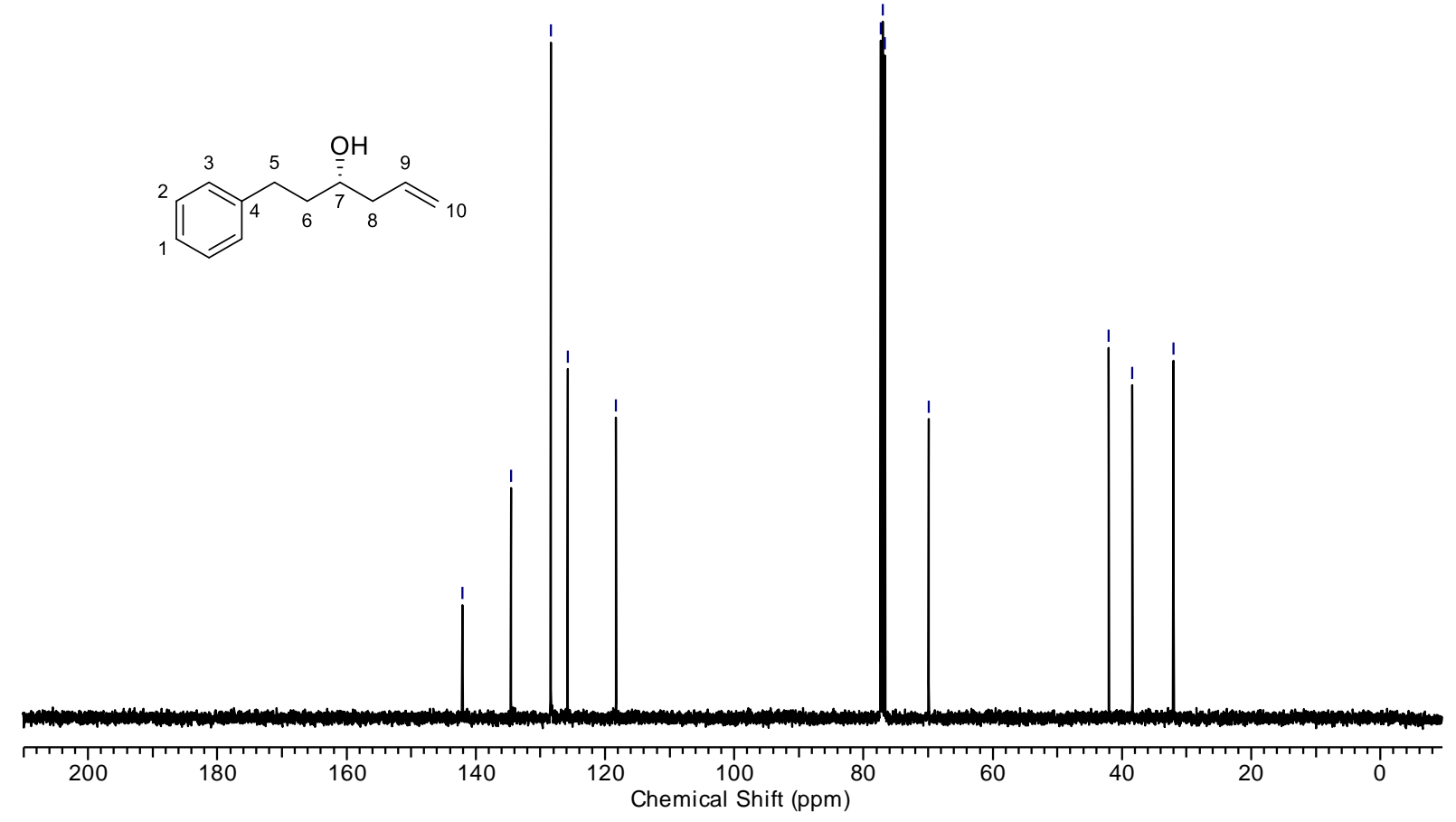




\section{HPLC (3)}

Column: Daicel Chiralcel OD-H (250 x $4.6 \mathrm{~mm}, 5 \mu \mathrm{m})$

Eluent: hexane/iPrOH 9:1, $1.0 \mathrm{ml} / \mathrm{min}, 20{ }^{\circ} \mathrm{C}$

racemic sample of $\mathbf{3}$ :

Chrom Type: Fixed WL Chromatogram, $210 \mathrm{~nm}$

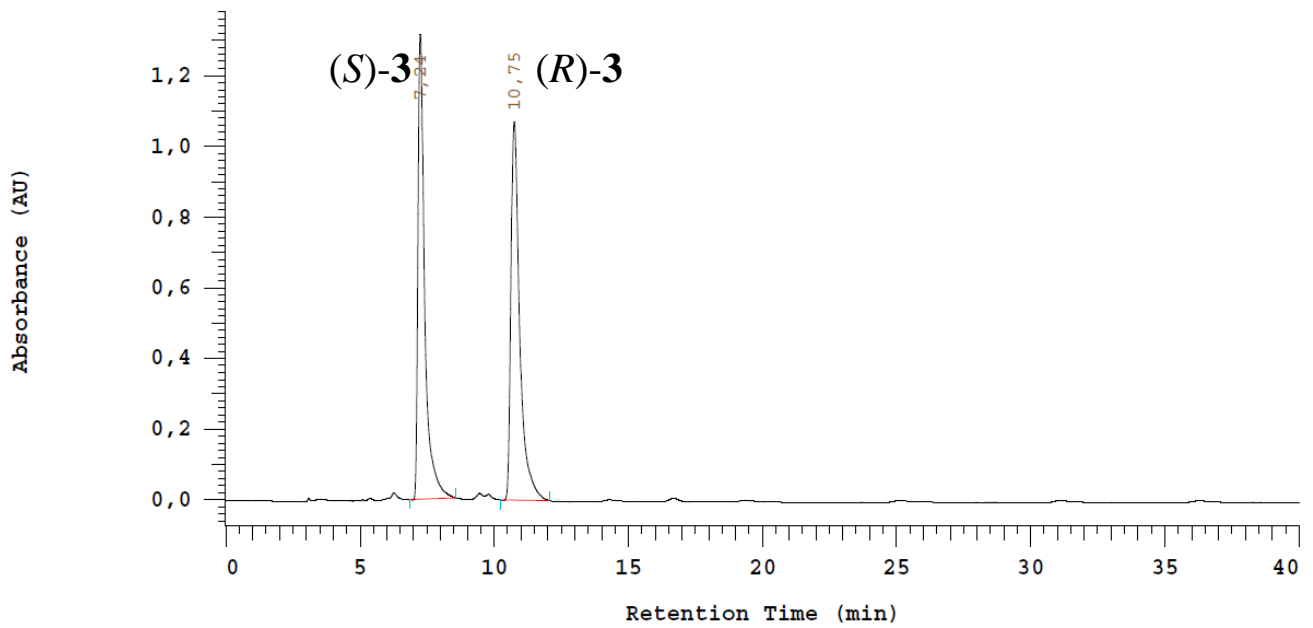

\begin{tabular}{rrrr} 
No. & \multicolumn{1}{c}{ RT } & Area & Area $\%$ \\
\hline 1 & 7,24 & 11556952 & 48,664 \\
2 & 10,75 & 12191588 & 51,336 \\
\hline & & 23748540 & 100,000 \\
\hline
\end{tabular}

3 prepared from boronic ester 2 by oxidation:

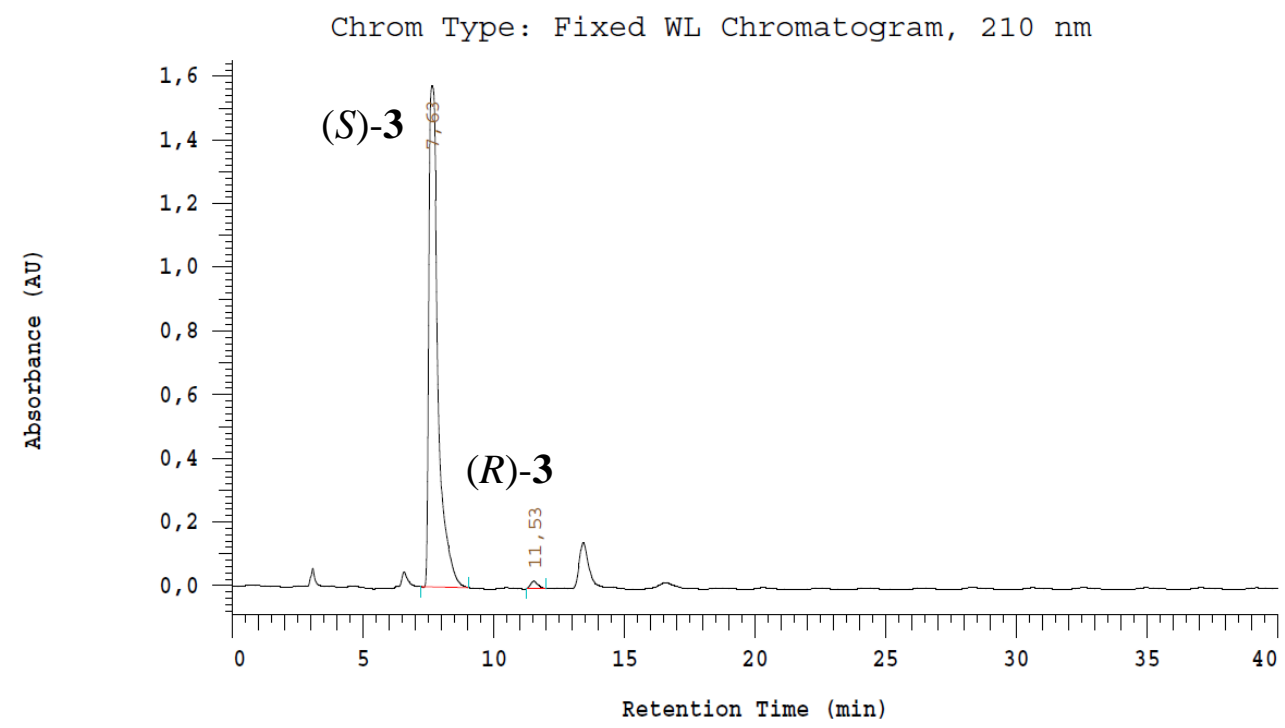

\begin{tabular}{rrrr} 
No. & \multicolumn{1}{c}{ RT } & \multicolumn{1}{c}{ Area } & Area \% \\
\hline 1 & 7,63 & 19132662 & 98,810 \\
2 & 11,53 & 230368 & 1,190 \\
\hline & & 19363030 & 100,000
\end{tabular}


(4S,5S)-4,5-Dicyclohexyl-2-[(S,Z)-1-phenylhept-5-en-3-yl]-1,3,2-dioxaborolane (5, mixture of $(E)$ and $(Z)$ isomers)

(4S,5S)-4,5-Dicyclohexyl-2-[(3S)-4-methyl-1-phenylhex-5-en-3-yl]-1,3,2-dioxaborolane

(6)

${ }^{1} \mathbf{H}$ NMR $\left(400 \mathrm{MHz}, \mathrm{CDCl}_{3}\right)$ :

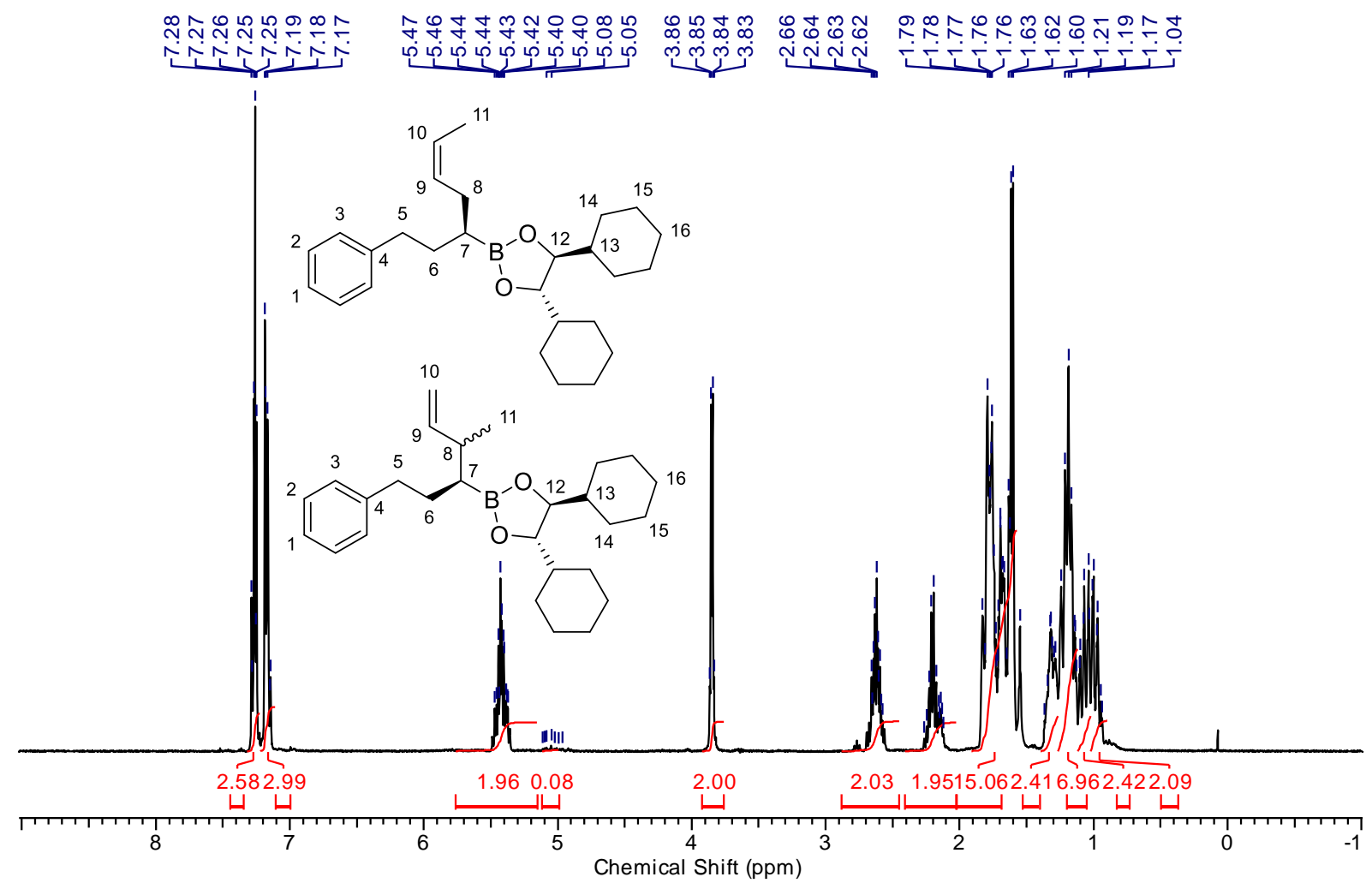

${ }^{13} \mathrm{C}$ NMR $\left(100 \mathrm{MHz}, \mathrm{CDCl}_{3}\right)$ :

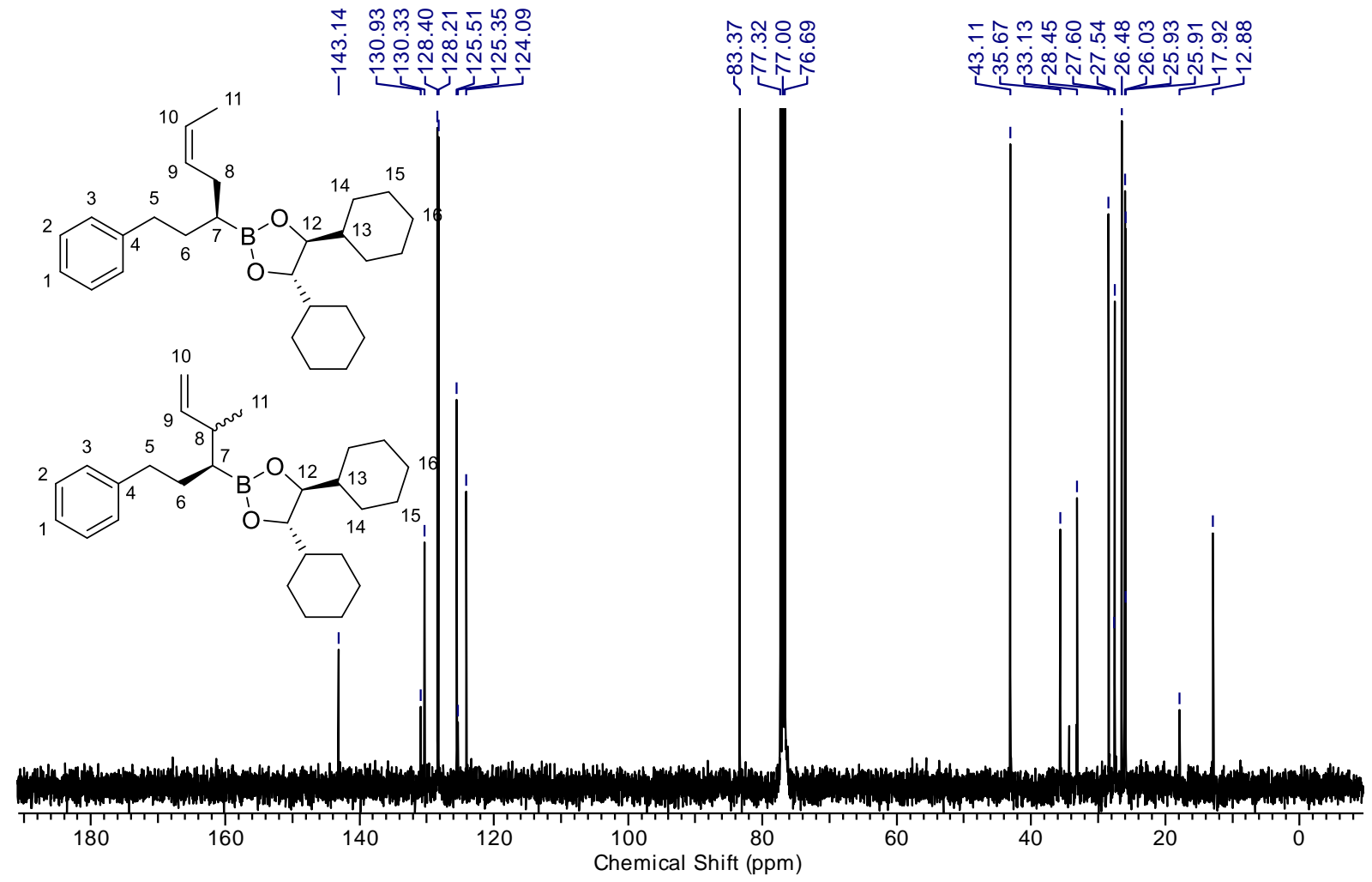


$(S, Z)$-1-Phenylhept-5-en-3-ol (5', mixture of $(E)$ and $(Z)$ isomers)

${ }^{1} \mathbf{H}$ NMR (400 MHz, $\left.\mathrm{CDCl}_{3}\right)$ :

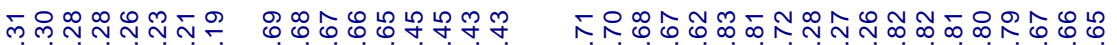

ن

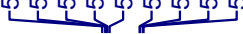

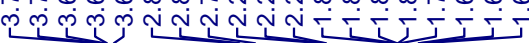
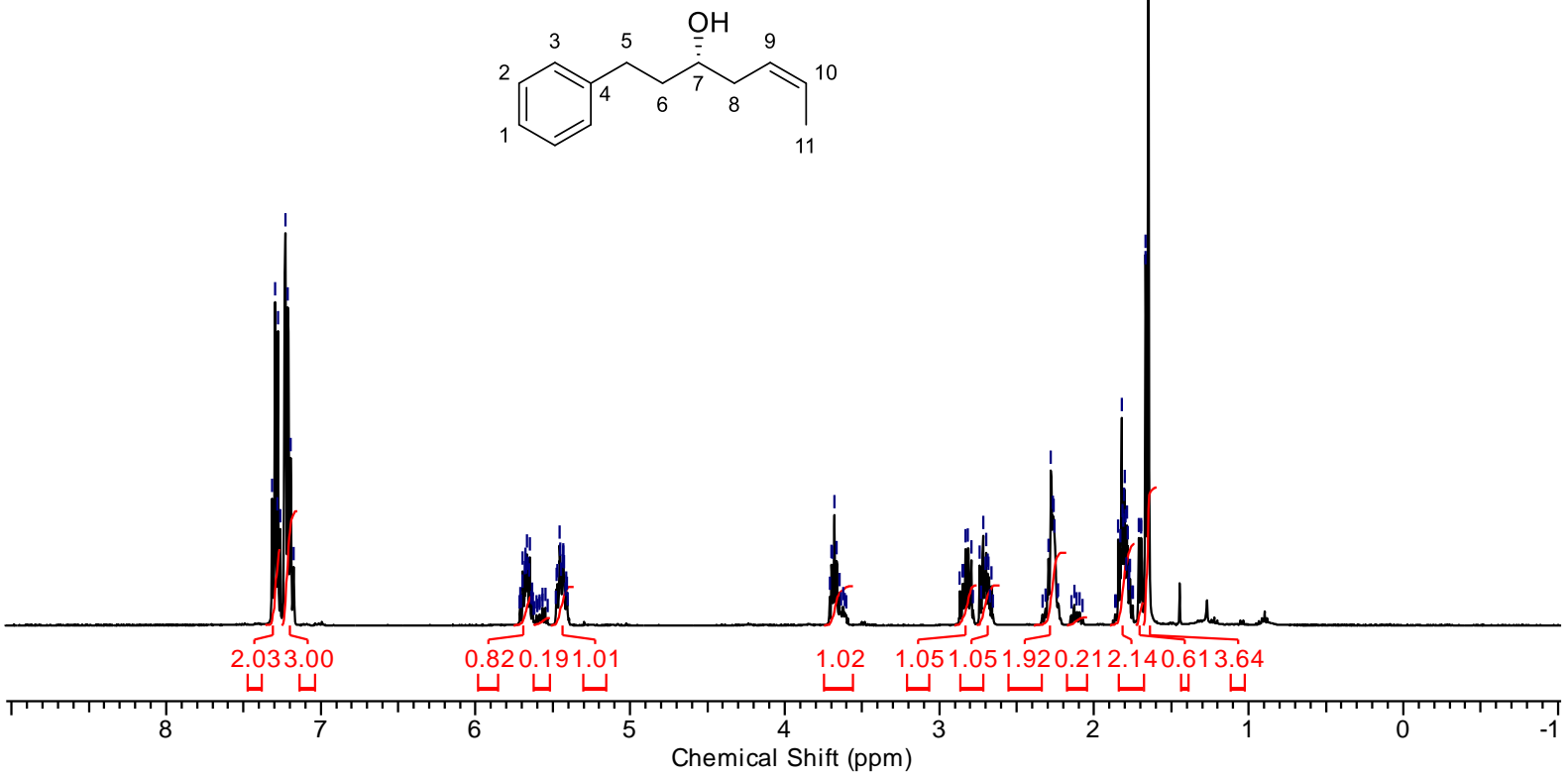

M01: 5.67 ppm; dqt; J=10.9, 6.8x(3), 1.3x(2); 4H

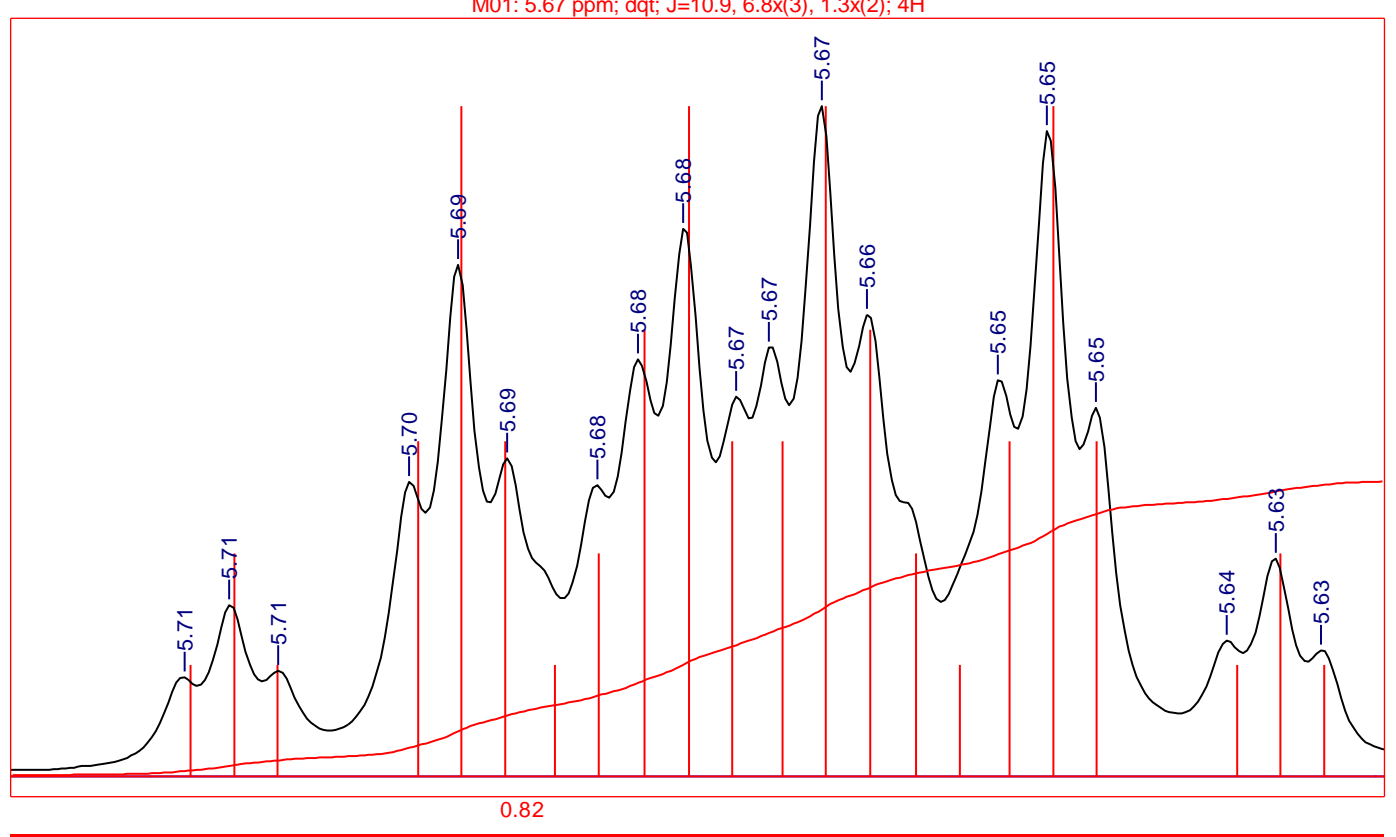




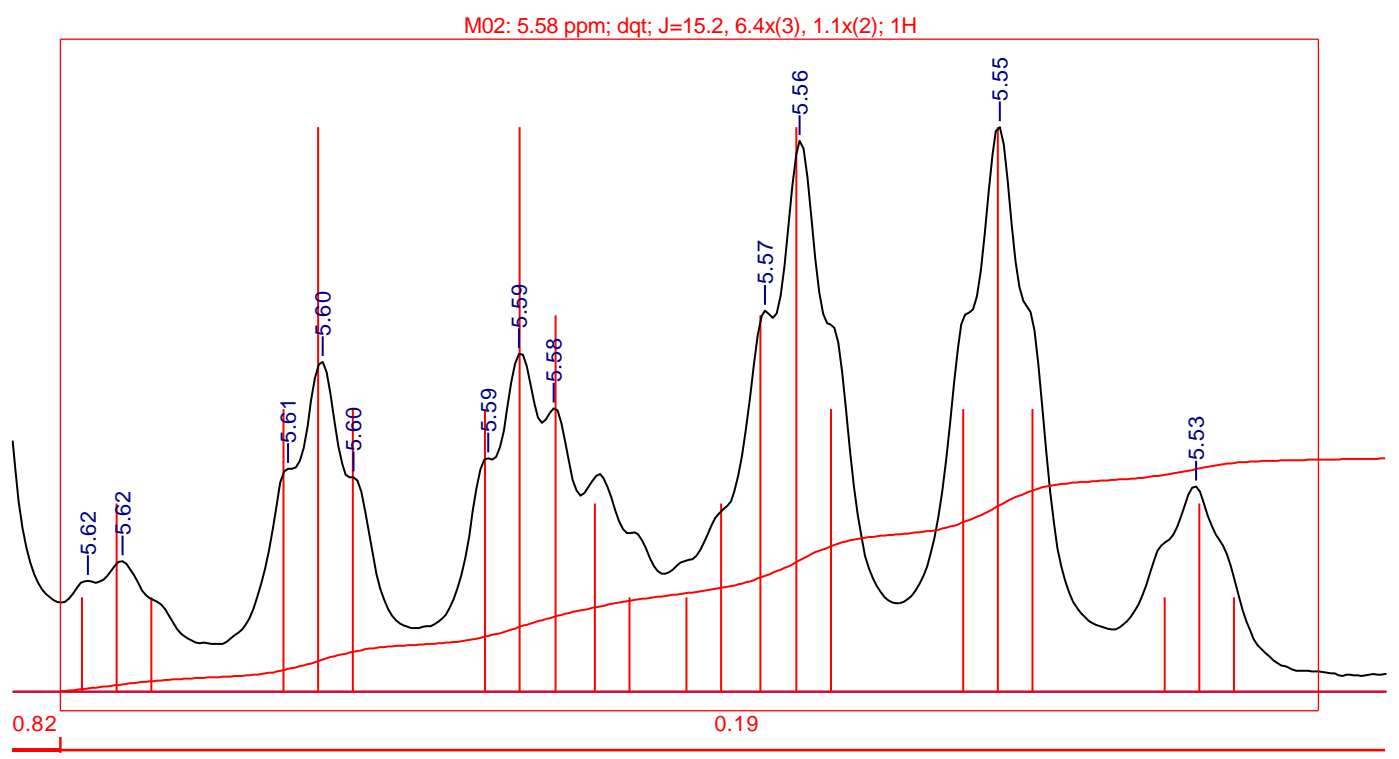

${ }^{13}$ C NMR (100 MHz, $\left.\mathrm{CDCl}_{3}\right)$ :

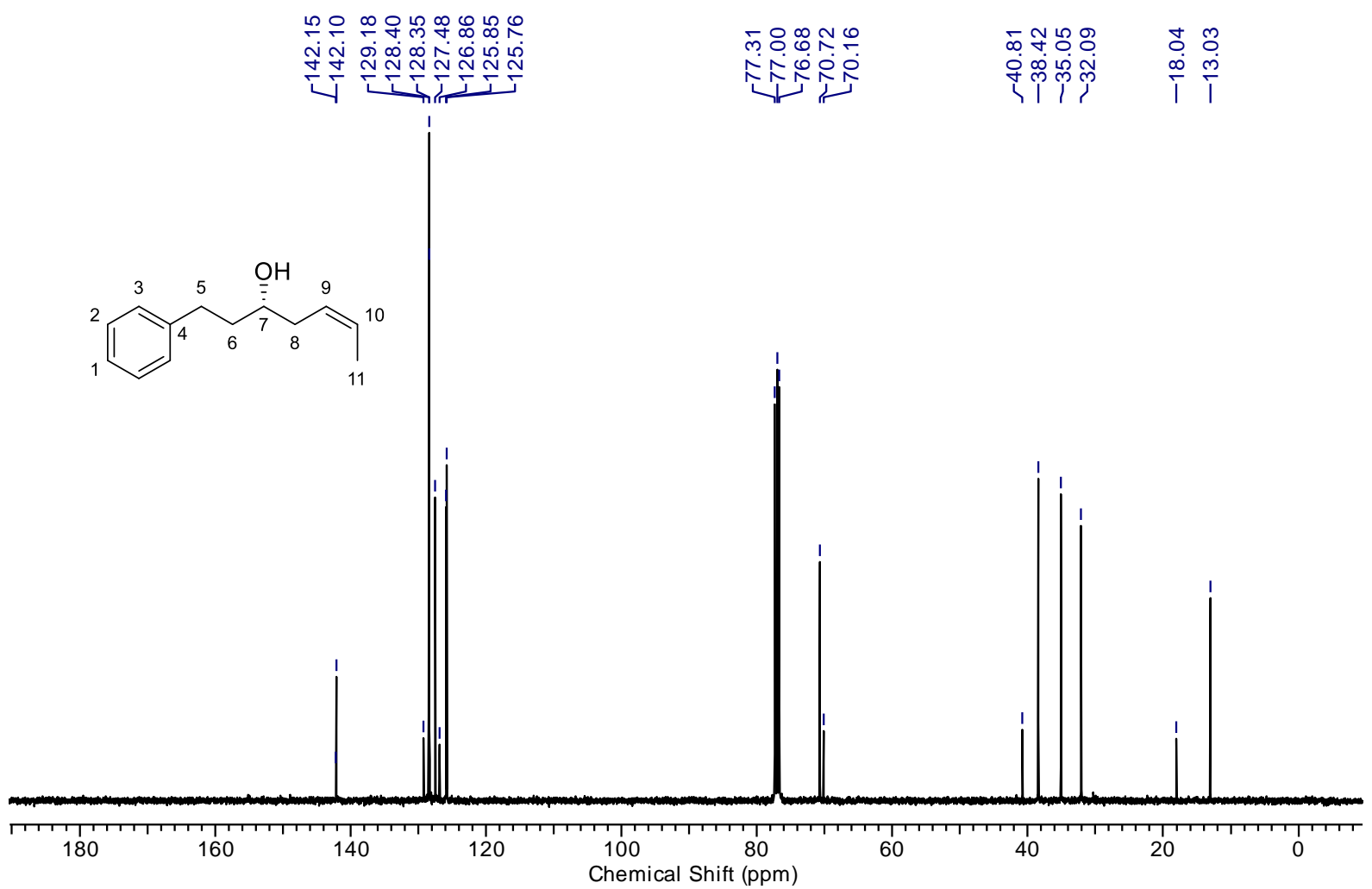


(4S,5S)-4,5-Dicyclohexyl-2-[(S)-5-methyl-1-phenylhex-5-en-3-yl]-1,3,2-dioxaborolane (10a)

${ }^{1} \mathbf{H}$ NMR $\left(400 \mathrm{MHz}, \mathrm{CDCl}_{3}\right)$ :

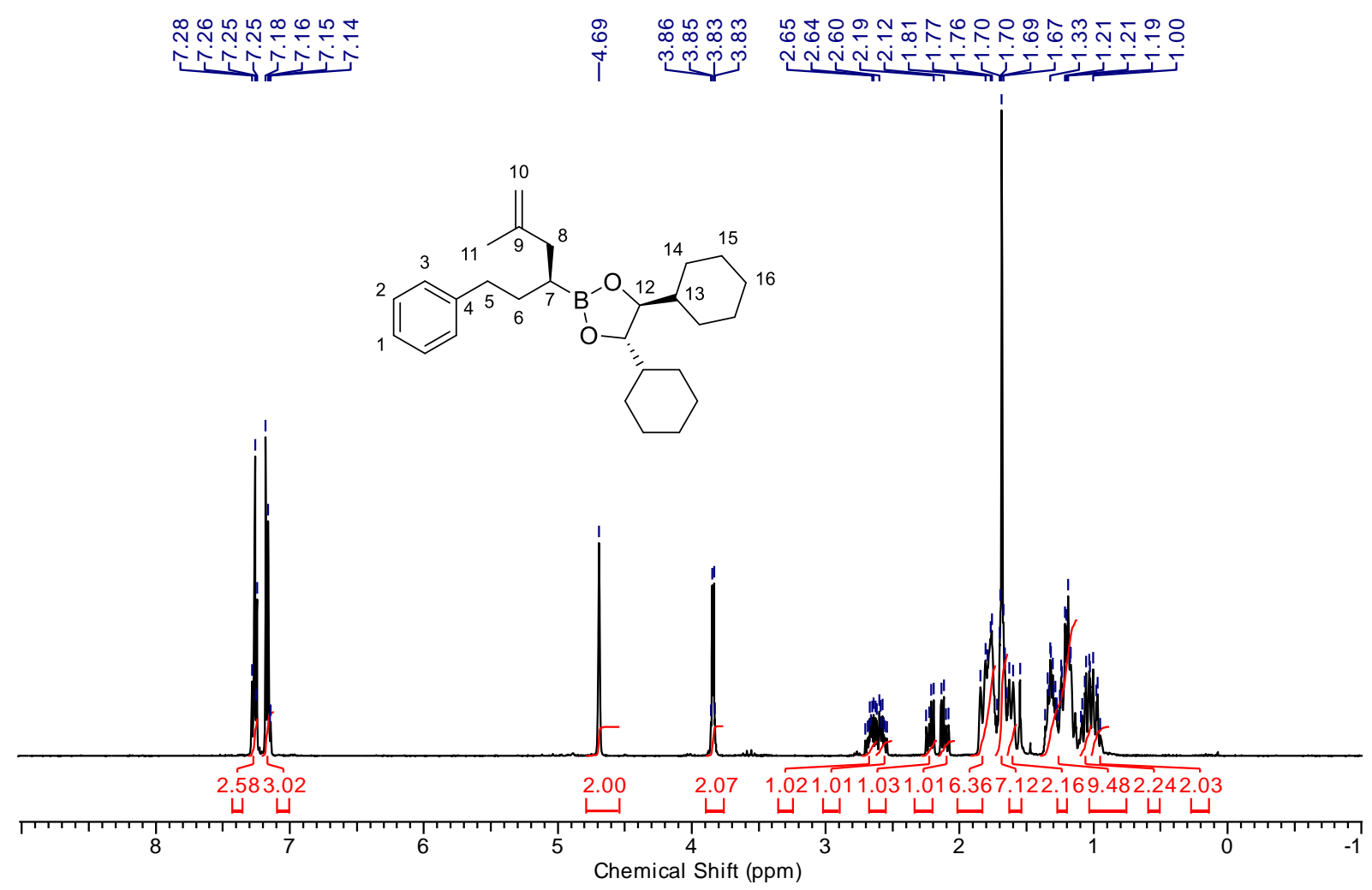

${ }^{13} \mathrm{C}$ NMR $\left(100 \mathrm{MHz}, \mathrm{CDCl}_{3}\right)$ :

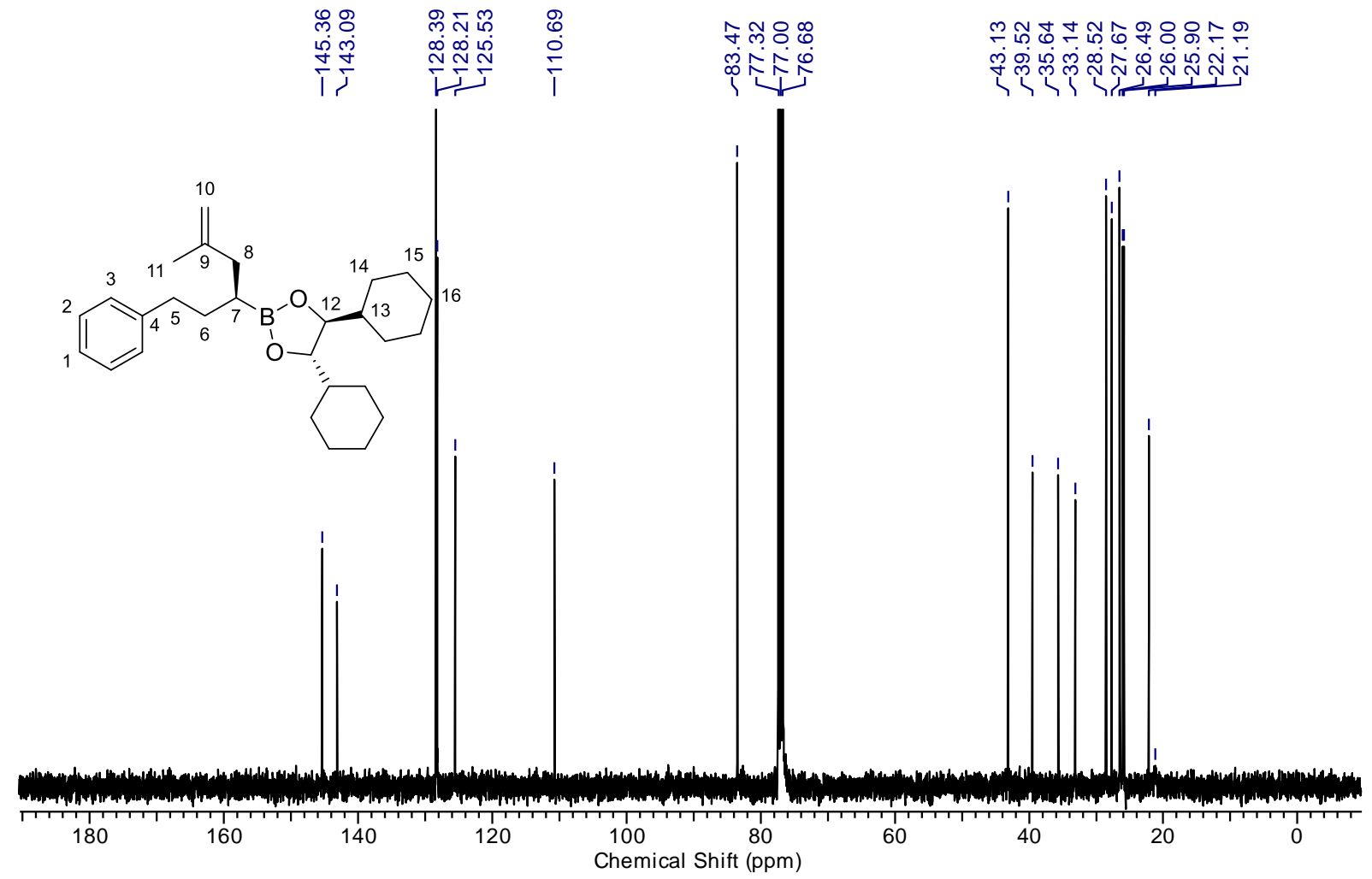


$(4 S, 5 S)-4,5-D i c y c l o h e x y l-2-[(S, Z)-1$-phenyloct-5-en-3-yl]-1,3,2-dioxaborolane (10b, mixture of $(E)$ and $(Z)$ isomers)

${ }^{1} \mathbf{H}$ NMR (500 MHz, $\left.\mathrm{CDCl}_{3}\right)$ :

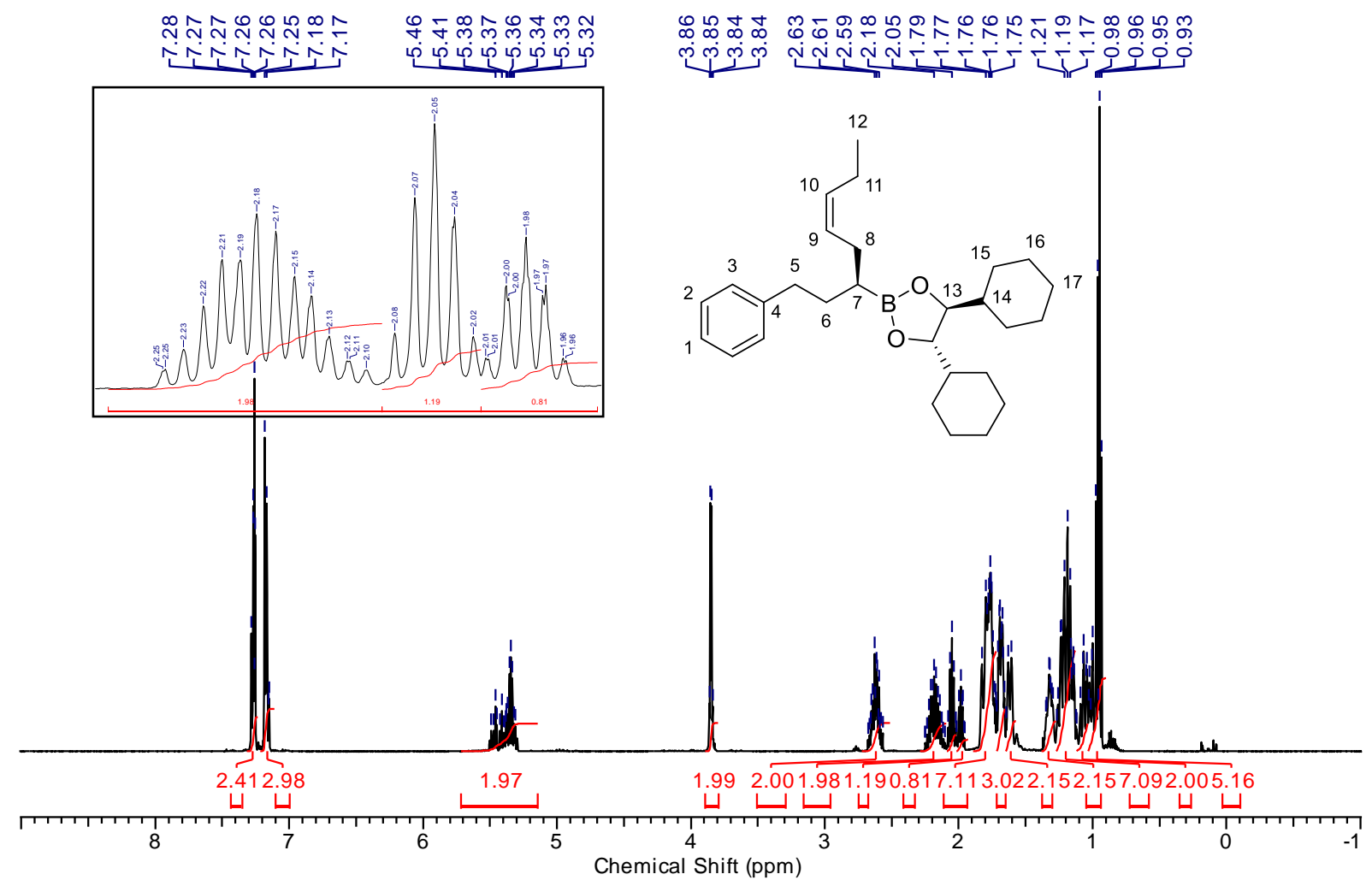

${ }^{13}$ C NMR (125 MHz, $\left.\mathrm{CDCl}_{3}\right)$ :

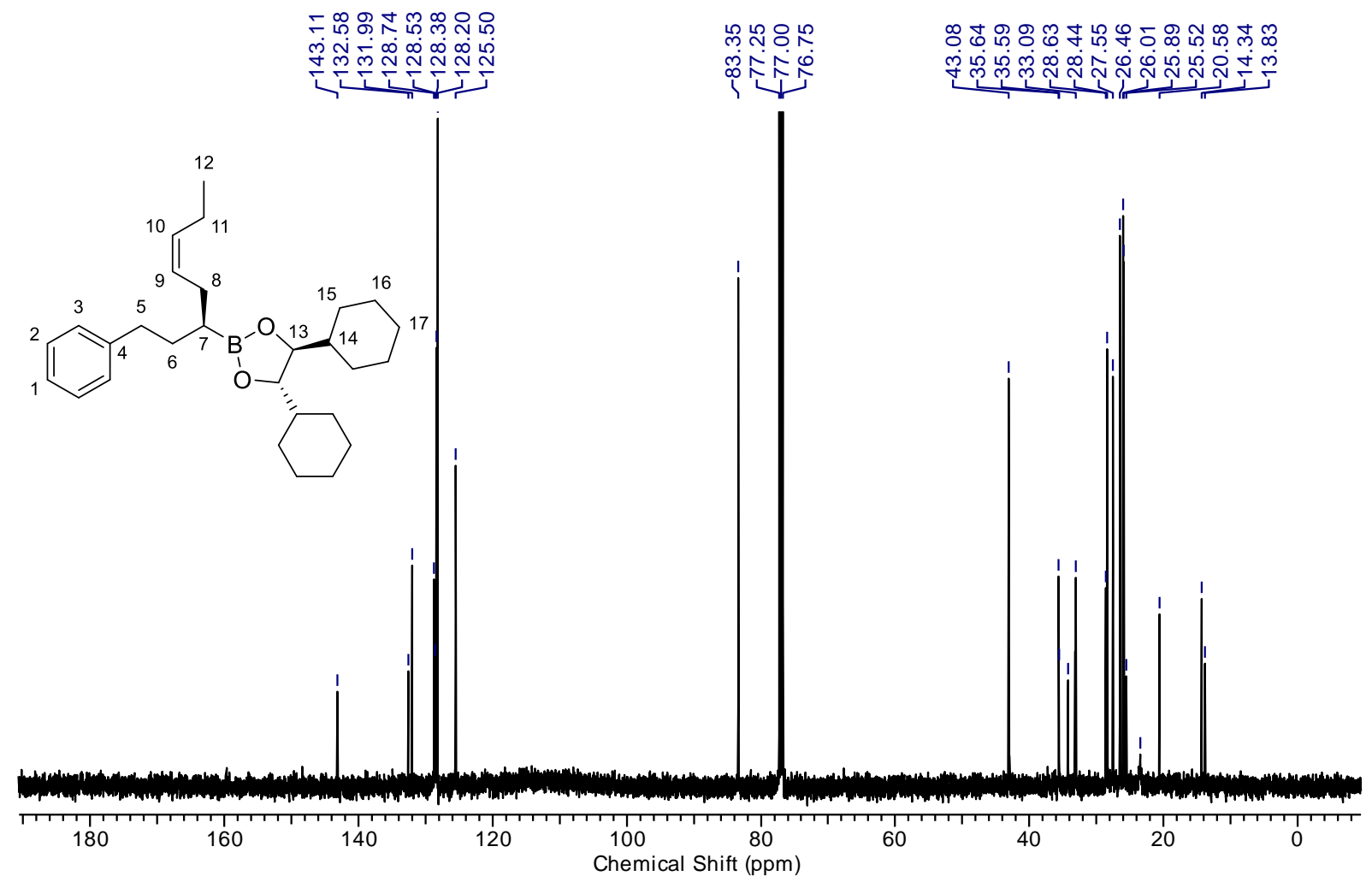


(4S,5S)-4,5-Dicyclohexyl-2-[(S,Z)-1-phenylnon-5-en-3-yl]-1,3,2-dioxaborolane (10c, mixture of $(E)$ and $(Z)$ isomers)

${ }^{1} \mathbf{H}$ NMR (500 MHz, $\left.\mathrm{CDCl}_{3}\right)$ :

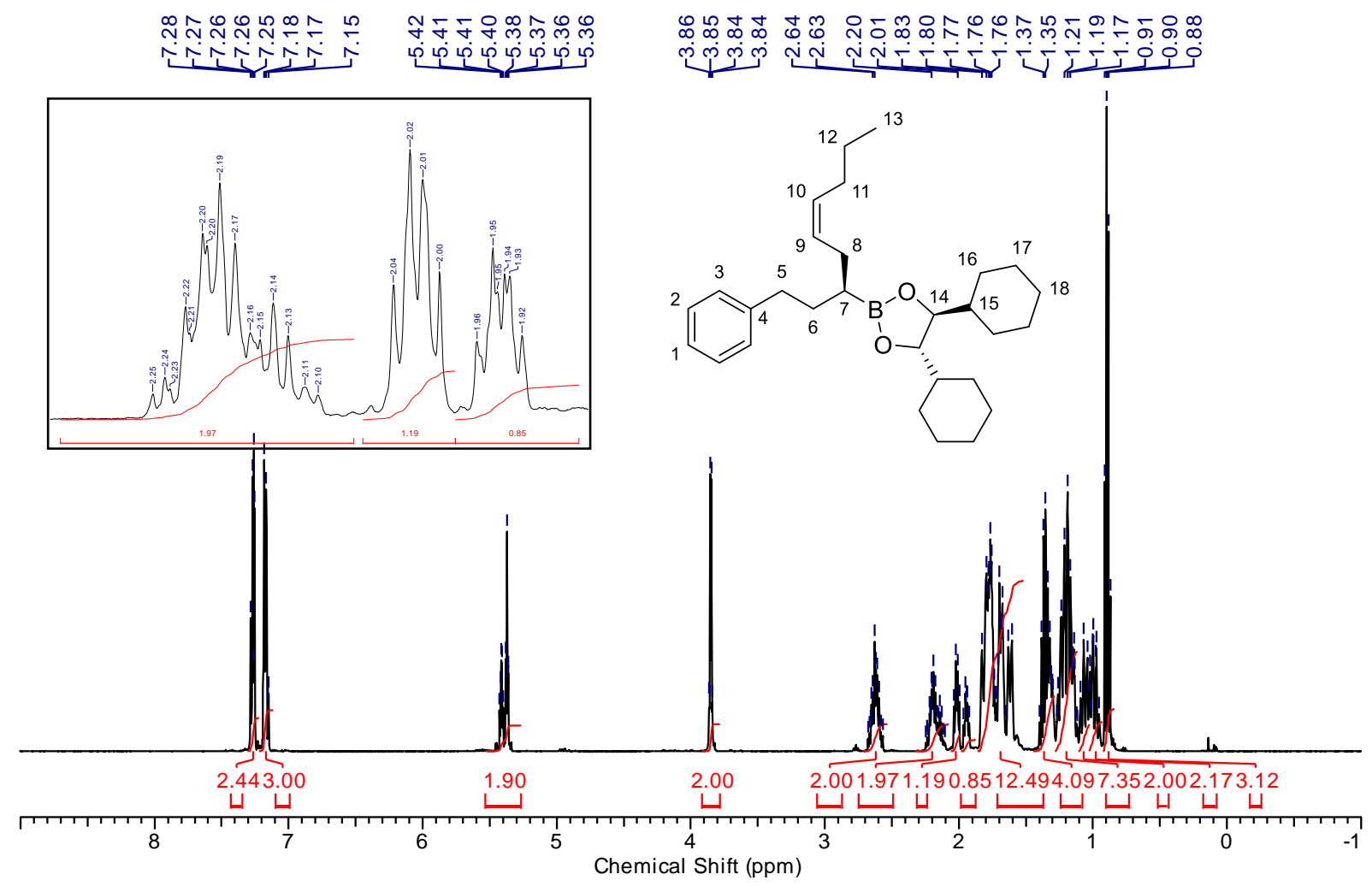

${ }^{13} \mathrm{C}$ NMR (125 MHz, $\left.\mathrm{CDCl}_{3}\right)$ :

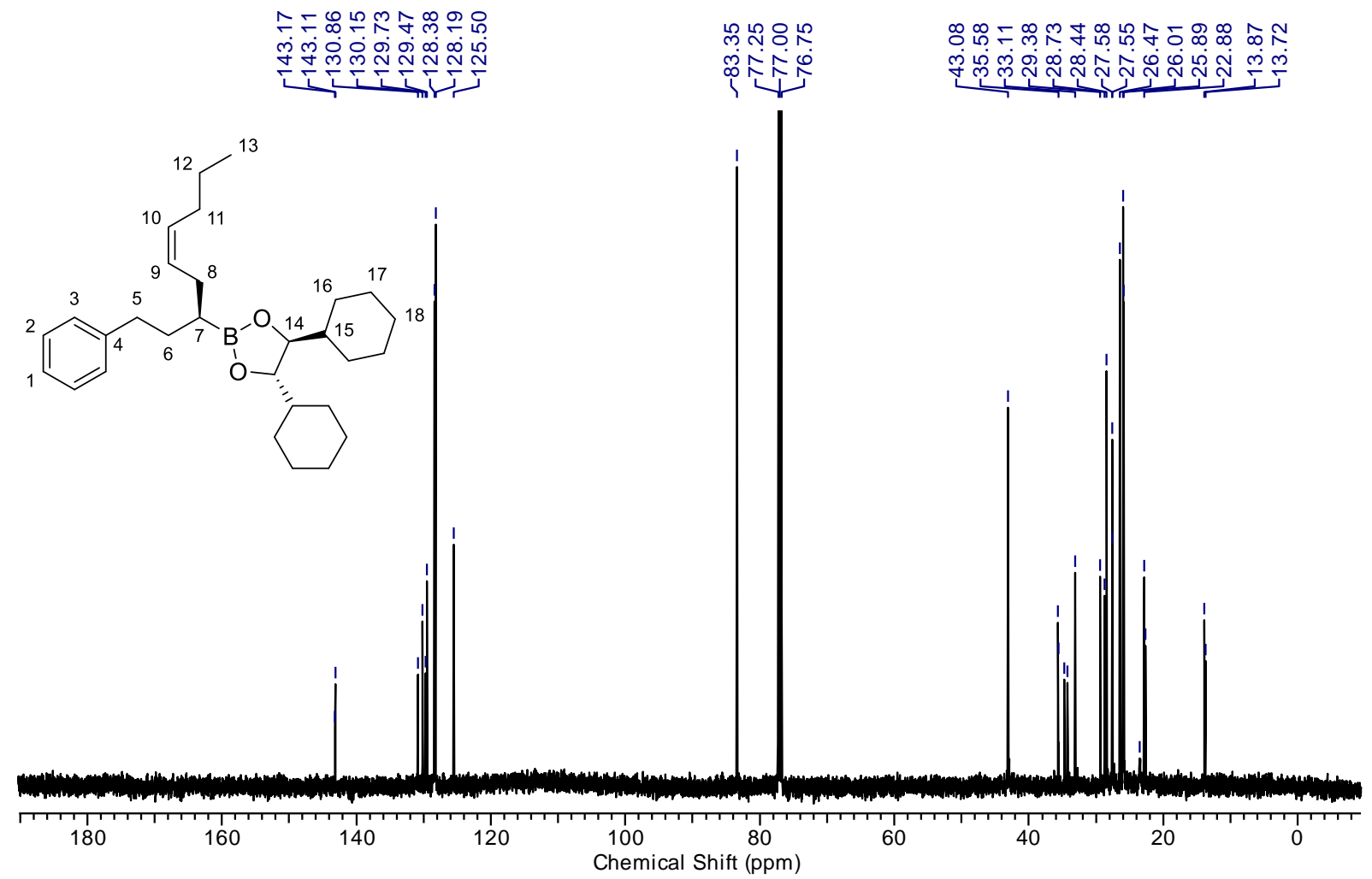


(4S,5S)-4,5-Dicyclohexyl-2-[(S,Z)-1-phenylundec-5-en-3-yl]-1,3,2-dioxaborolane (10d, mixture of $(E)$ and $(Z)$ isomers)

${ }^{1} \mathbf{H}$ NMR $\left(500 \mathrm{MHz}, \mathrm{CDCl}_{3}\right)$ :

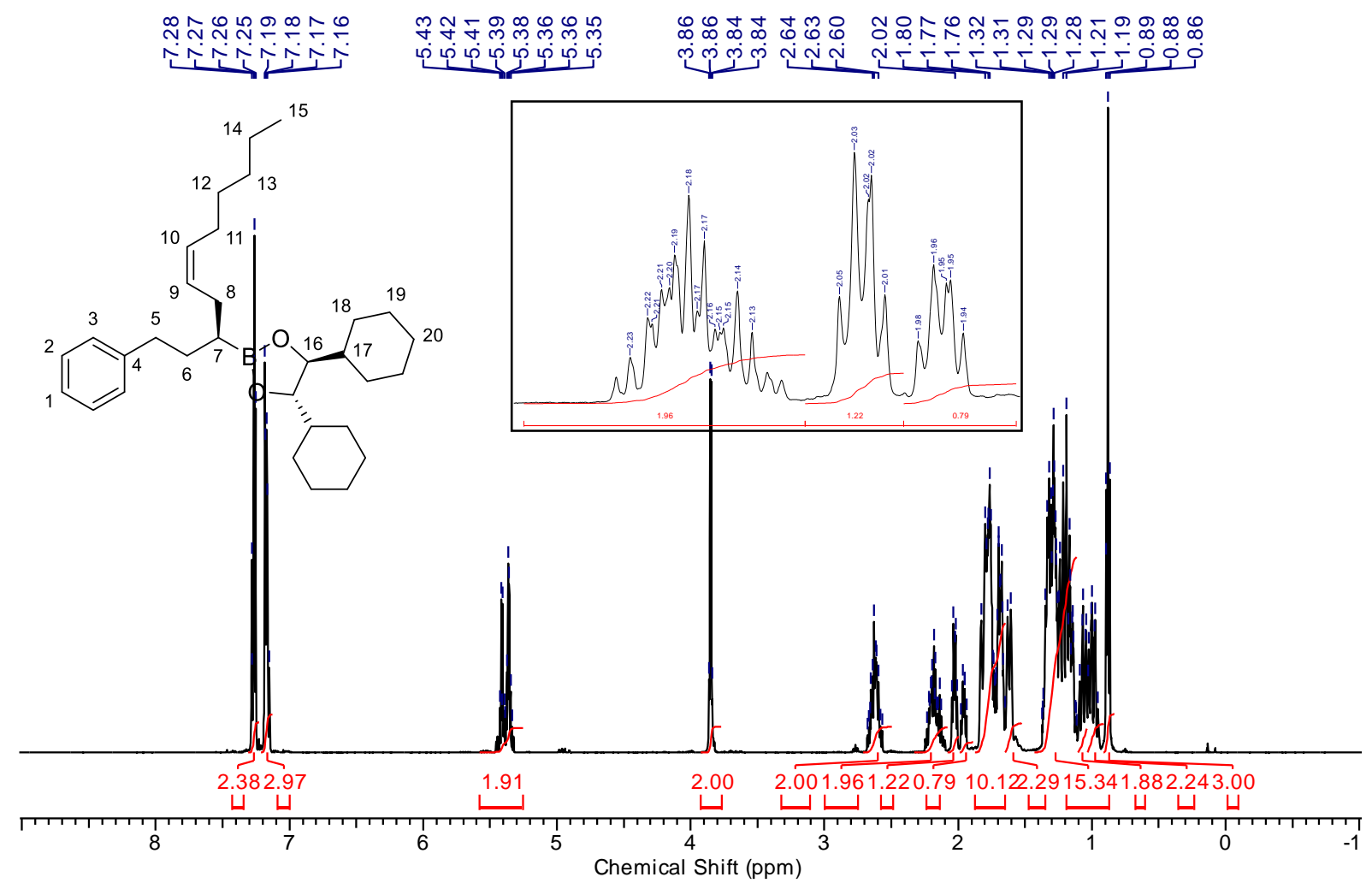

${ }^{13}$ C NMR (125 MHz, $\left.\mathrm{CDCl}_{3}\right)$ :

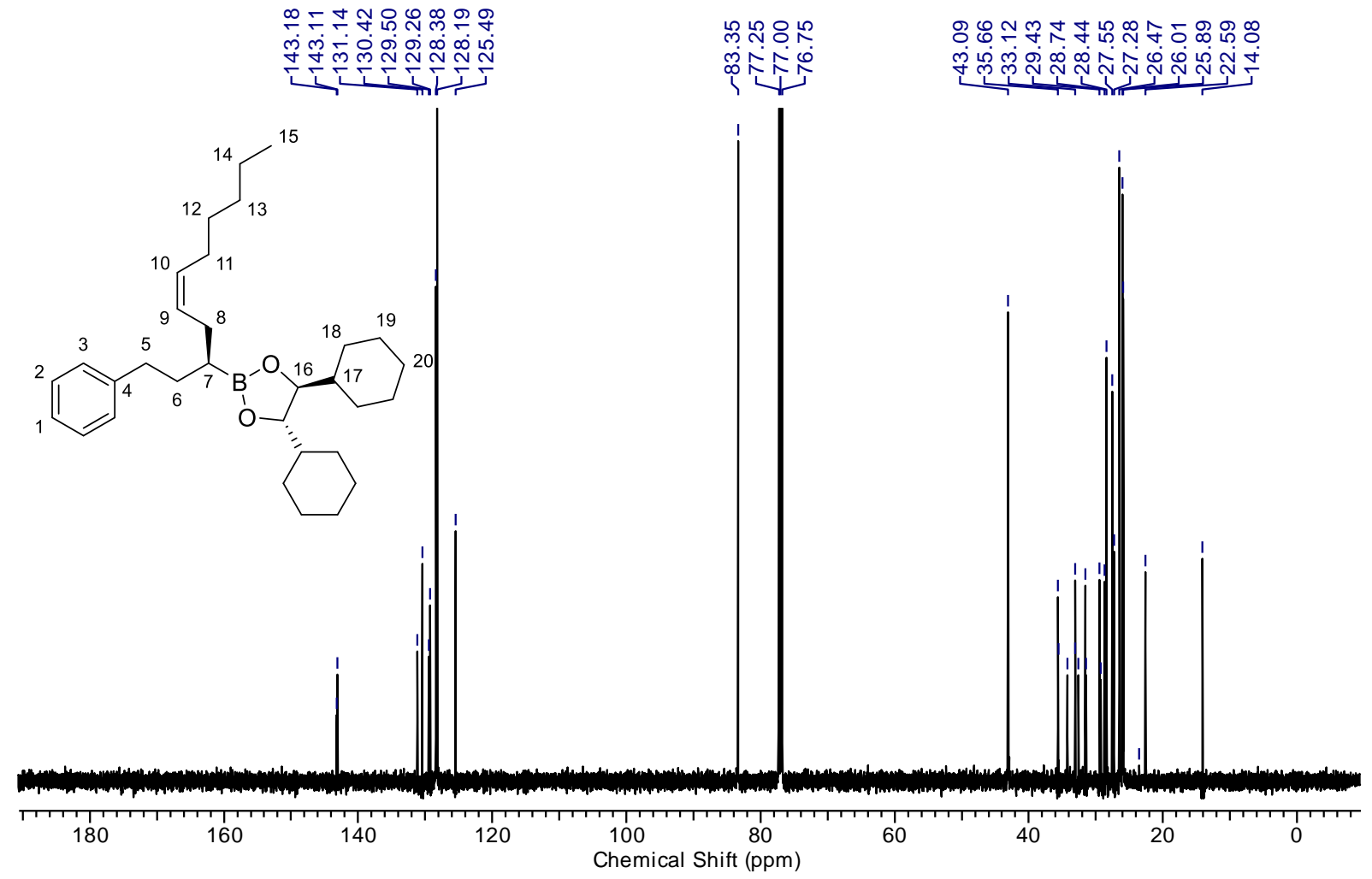


(S,Z)-1-Phenylundec-5-en-3-ol (10d', mixture of $(E)$ and $(Z)$ isomers)

${ }^{1} \mathbf{H}$ NMR (500 MHz, $\left.\mathrm{CDCl}_{3}\right)$ :

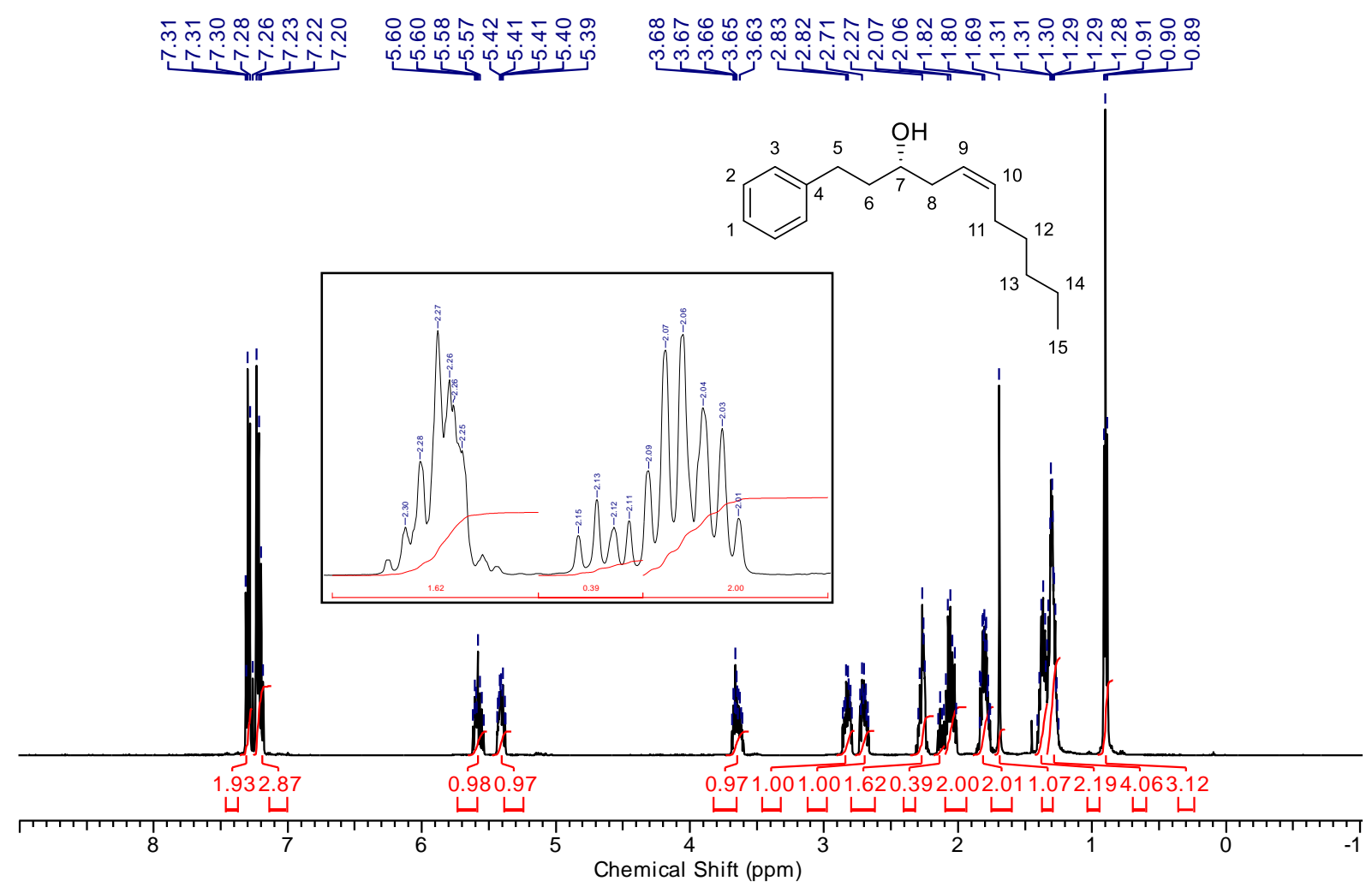

${ }^{13} \mathrm{C}$ NMR $\left(125 \mathrm{MHz}, \mathrm{CDCl}_{3}\right)$ :

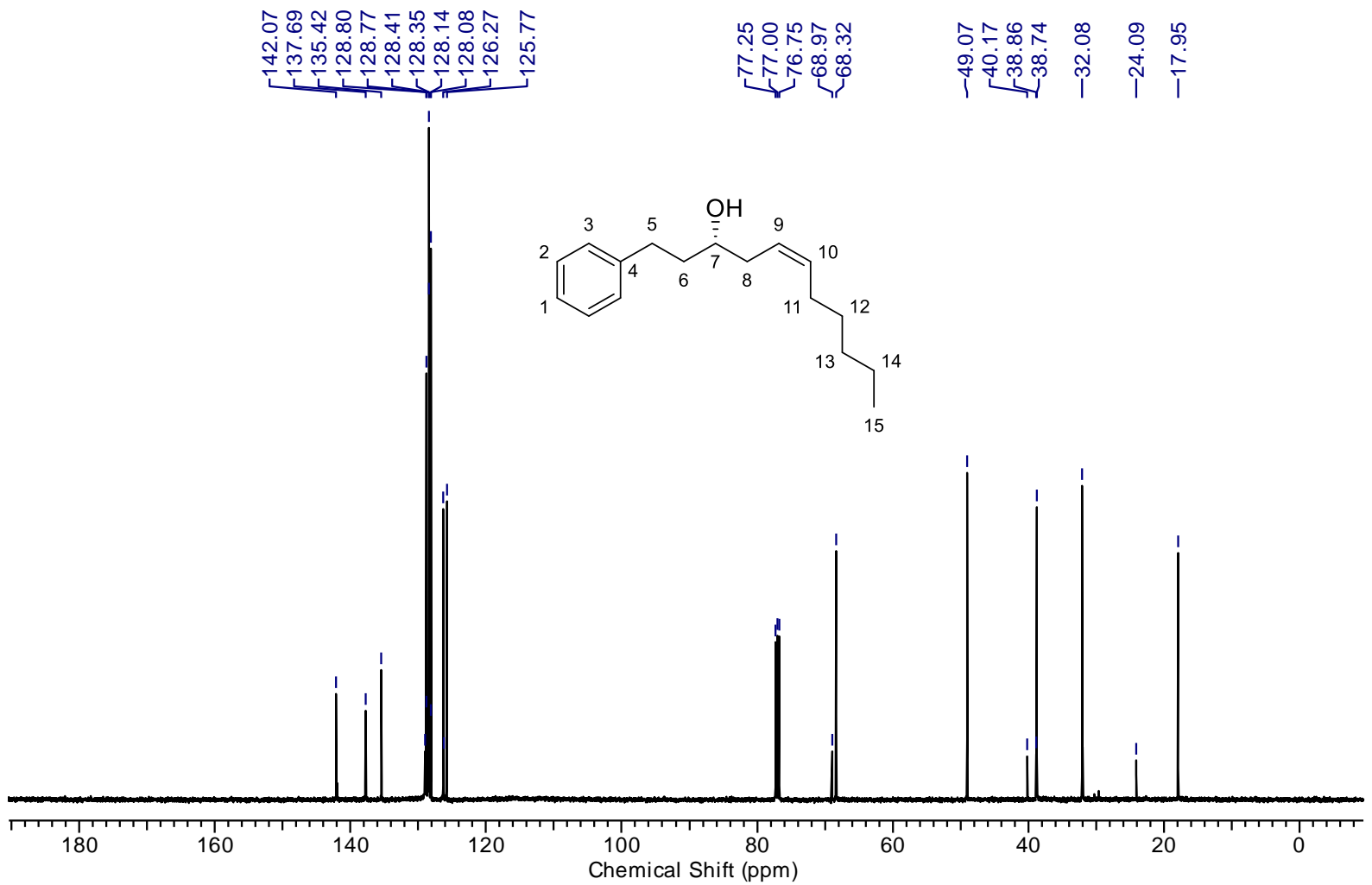


(4S,5S)-4,5-Dicyclohexyl-2-[(S,E)-7-methyl-1-phenyloct-5-en-3-yl]-1,3,2-dioxaborolane (10e, mixture of $(E)$ and $(Z)$ isomers)

${ }^{1} \mathbf{H}$ NMR (500 MHz, $\left.\mathrm{CDCl}_{3}\right)$ :

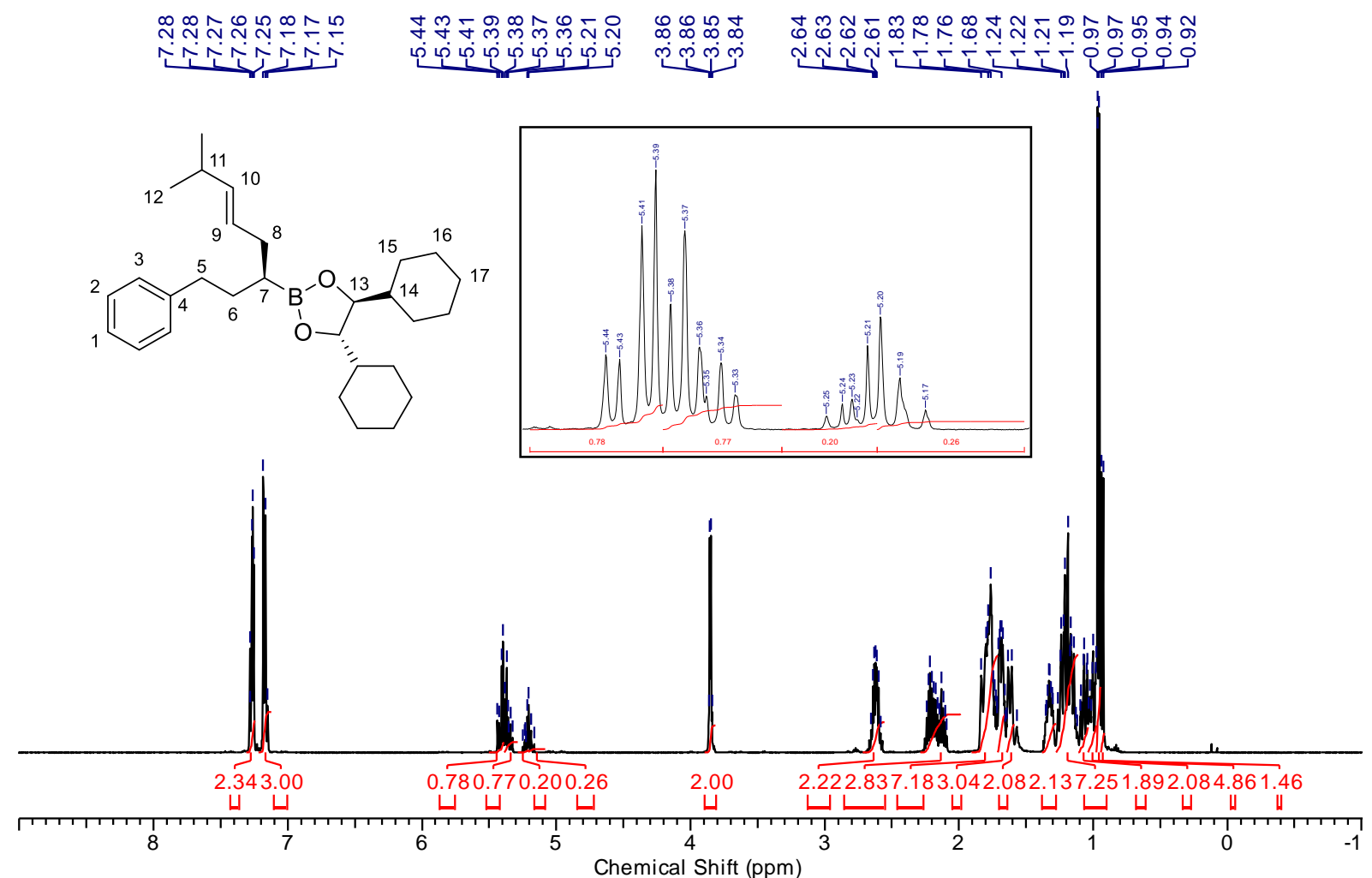

${ }^{13}$ C NMR (125 MHz, $\left.\mathrm{CDCl}_{3}\right)$ :

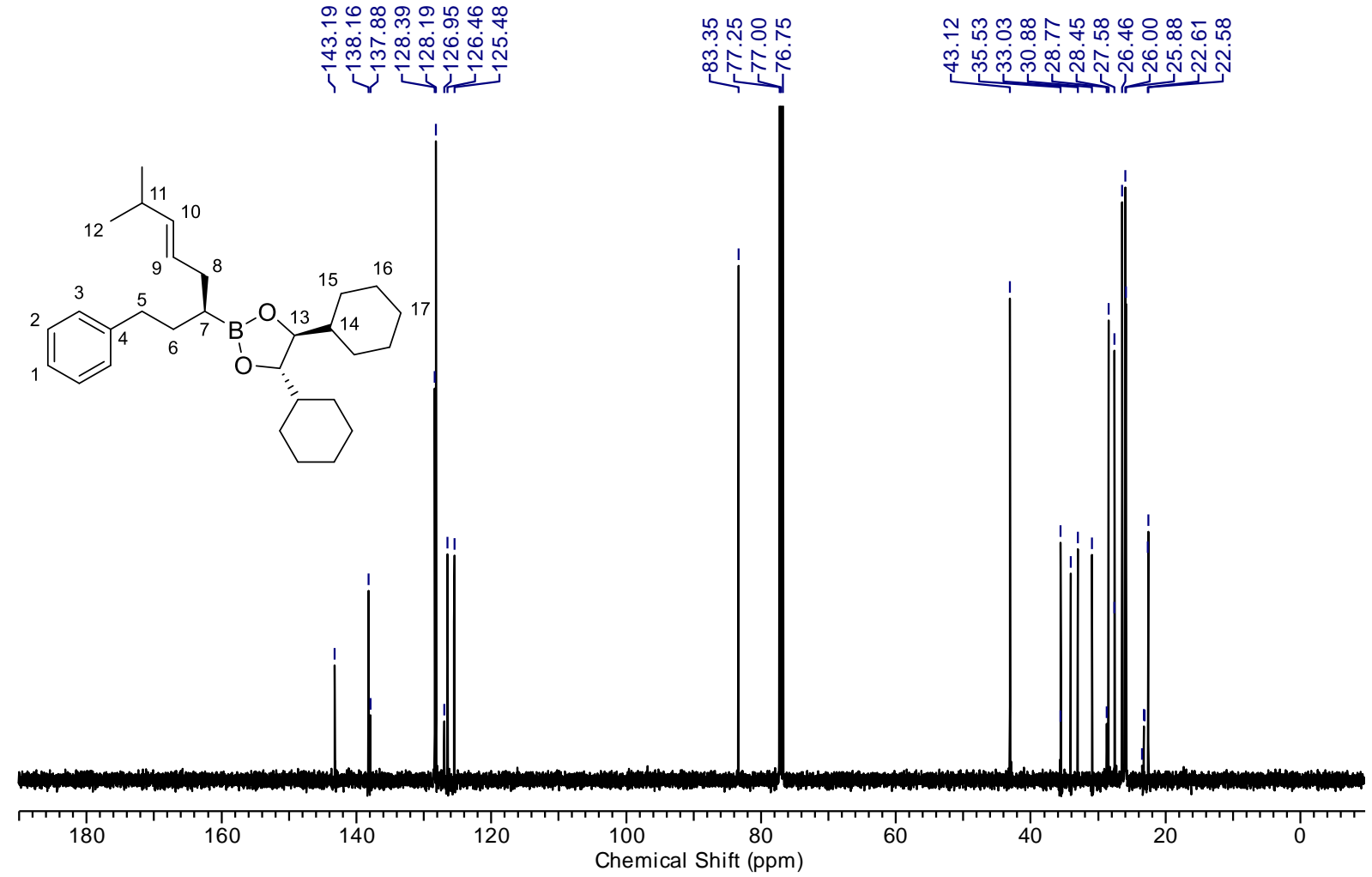


(4S,5S)-4,5-Dicyclohexyl-2-[(S,E)-7,7-dimethyl-1-phenyloct-5-en-3-yl]-1,3,2dioxaborolane (10f)

${ }^{1} \mathbf{H}$ NMR (500 MHz, $\left.\mathrm{CDCl}_{3}\right)$ :

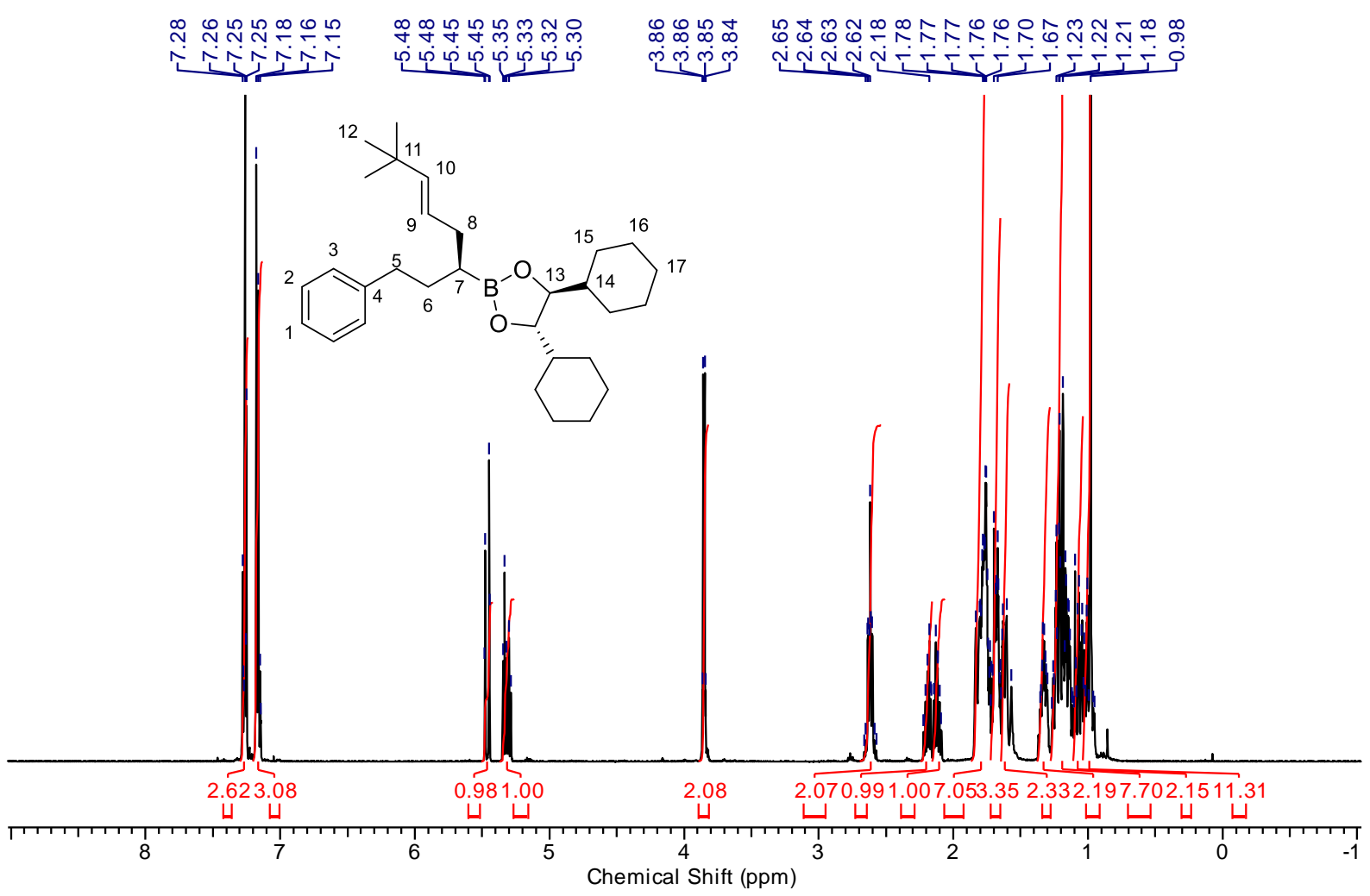

${ }^{13}$ C NMR (125 MHz, $\left.\mathrm{CDCl}_{3}\right)$ :

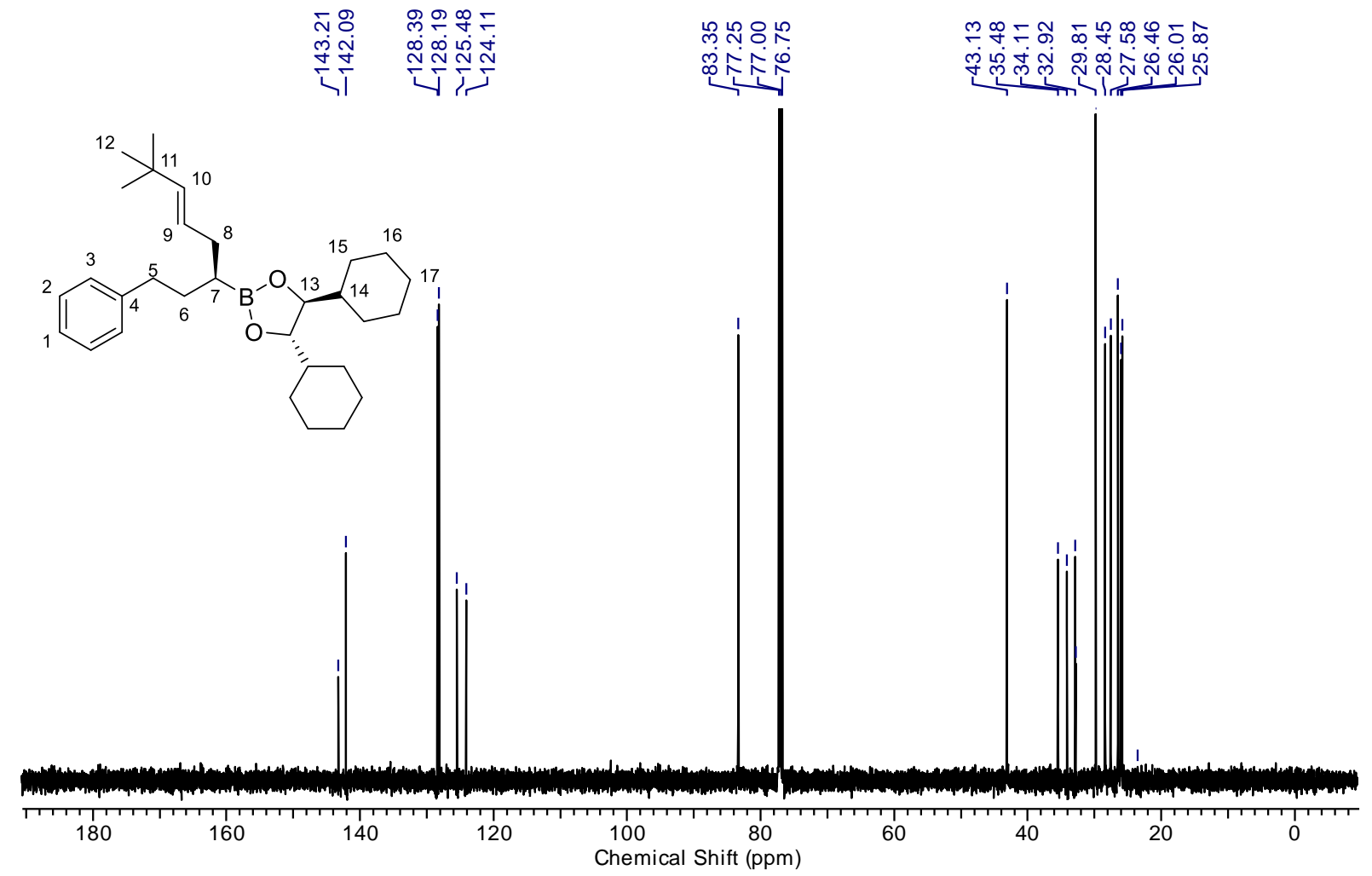


(4S,5S)-4,5-Dicyclohexyl-2-[(S,E)-1,6-diphenylhex-5-en-3-yl]-1,3,2-dioxaborolane (10g) ${ }^{1} \mathbf{H}$ NMR (500 MHz, $\left.\mathrm{CDCl}_{3}\right)$ :

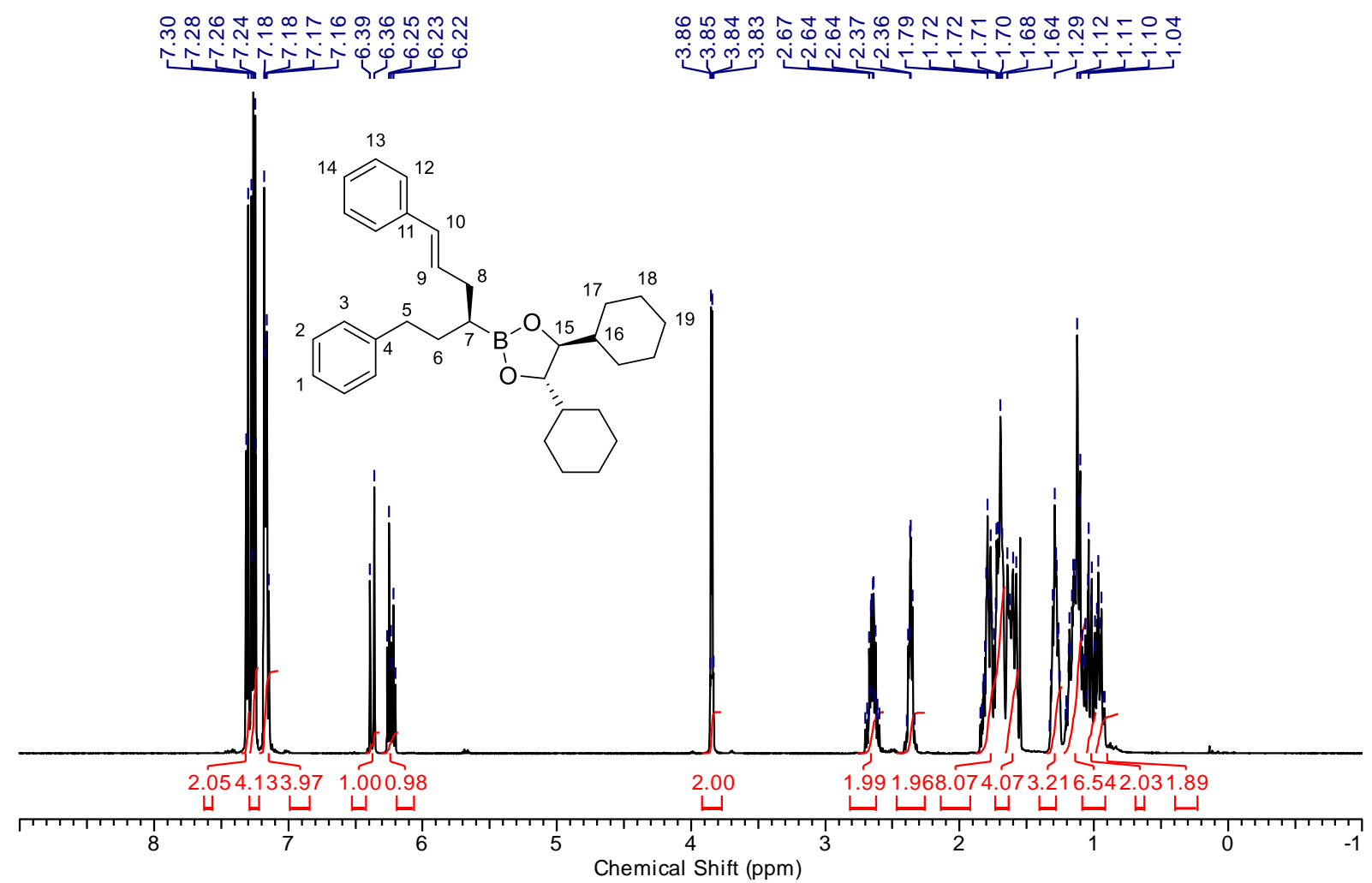

${ }^{13} \mathrm{C}$ NMR $\left(125 \mathrm{MHz}, \mathrm{CDCl}_{3}\right)$ :

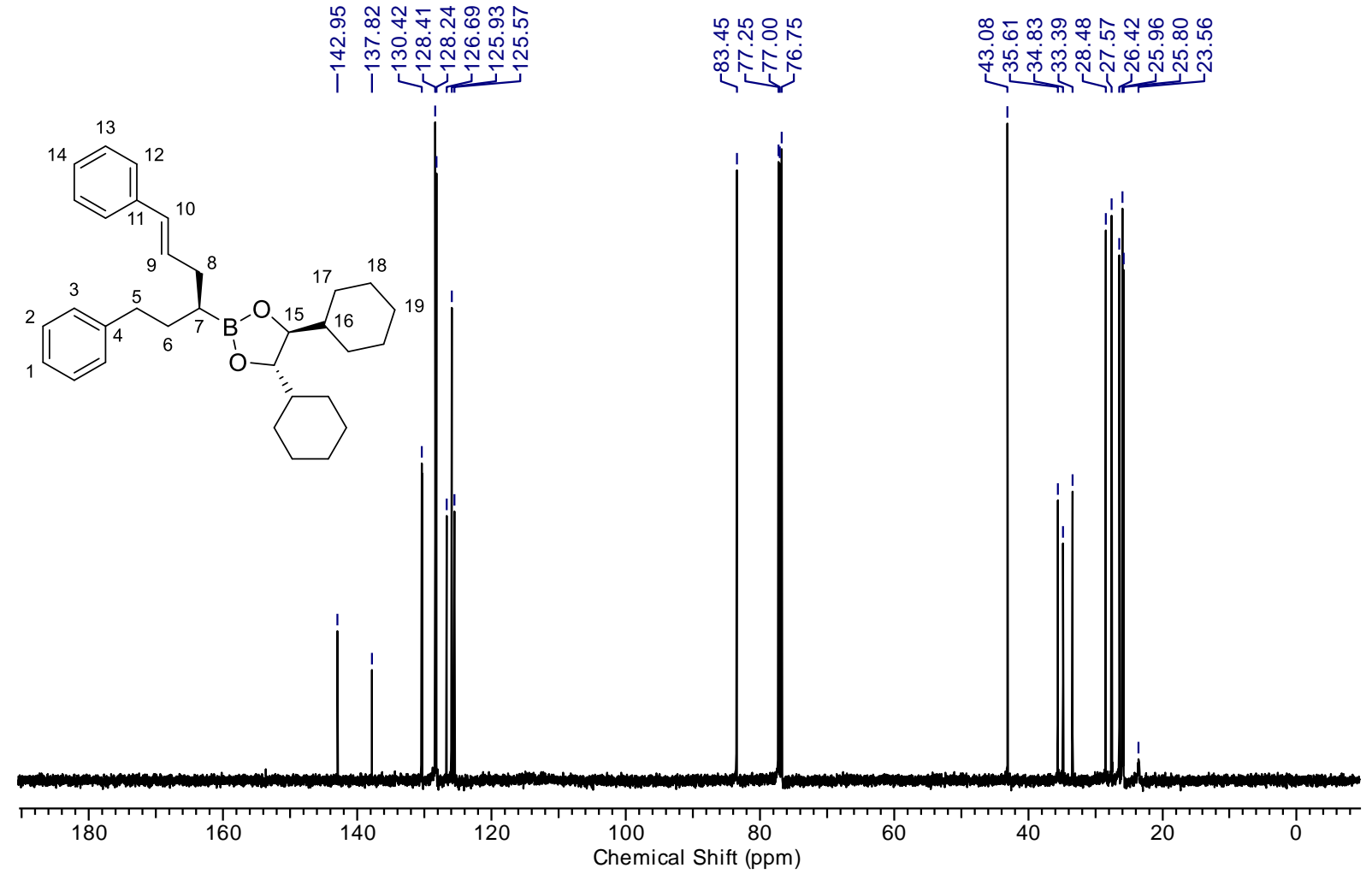


(4S,5S)-4,5-Dicyclohexyl-2-[(S,E)-5-methyl-1,6-diphenylhex-5-en-3-yl]-1,3,2dioxaborolane (10h, mixture of $(E)$ and $(Z)$ isomers)

${ }^{1} \mathbf{H}$ NMR (400 MHz, $\left.\mathrm{CDCl}_{3}\right)$ :

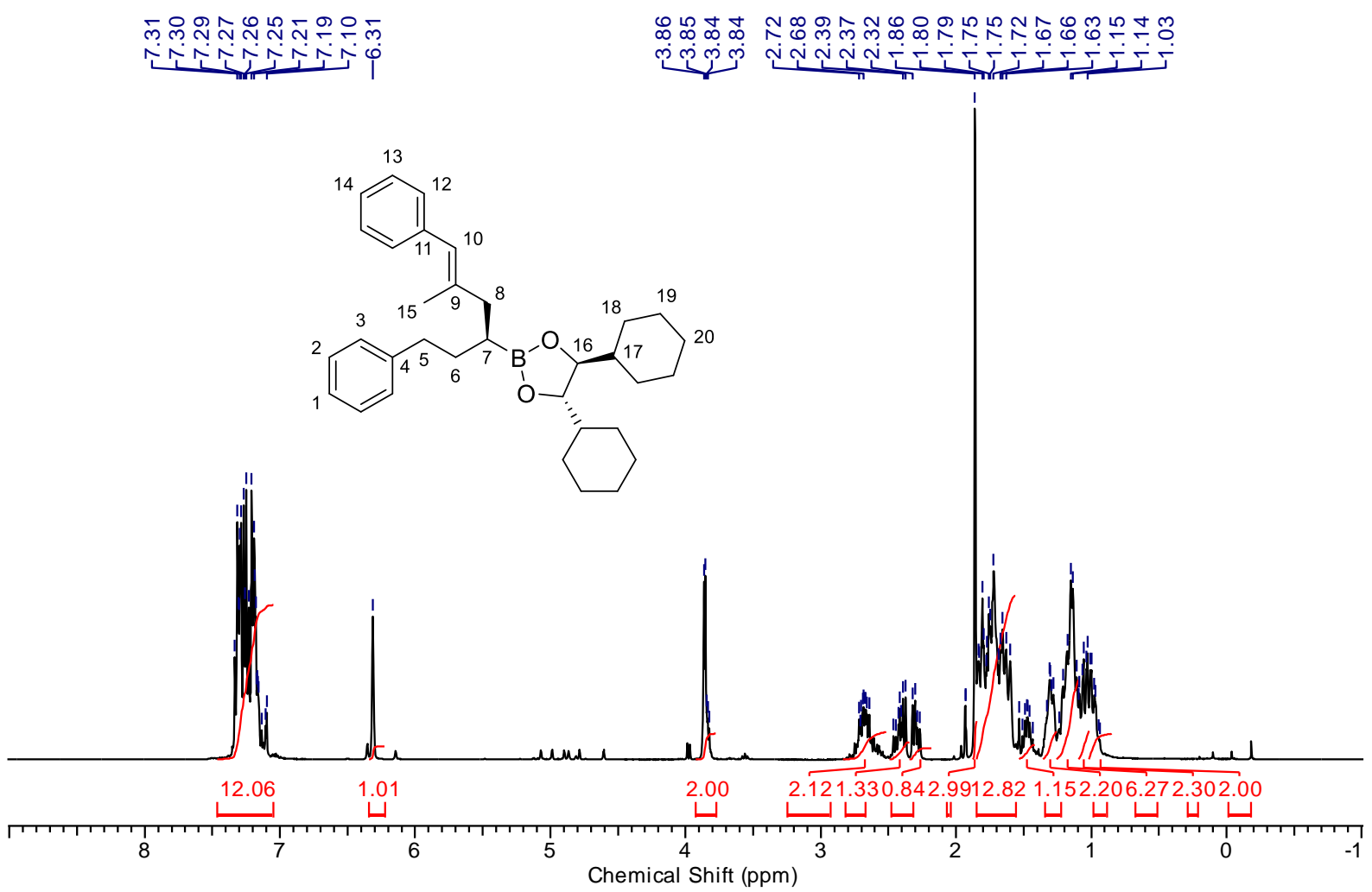

${ }^{13}$ C NMR (100 MHz, $\left.\mathrm{CDCl}_{3}\right)$ :

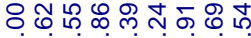

ঙำ

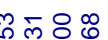

๓송

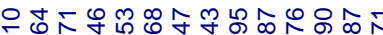

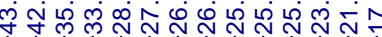<smiles>C/C(=C\c1ccccc1)C[C@H](CCc1ccccc1)B1OC([C]2[CH]CCCC2)C(C2CCCCC2)O1</smiles>
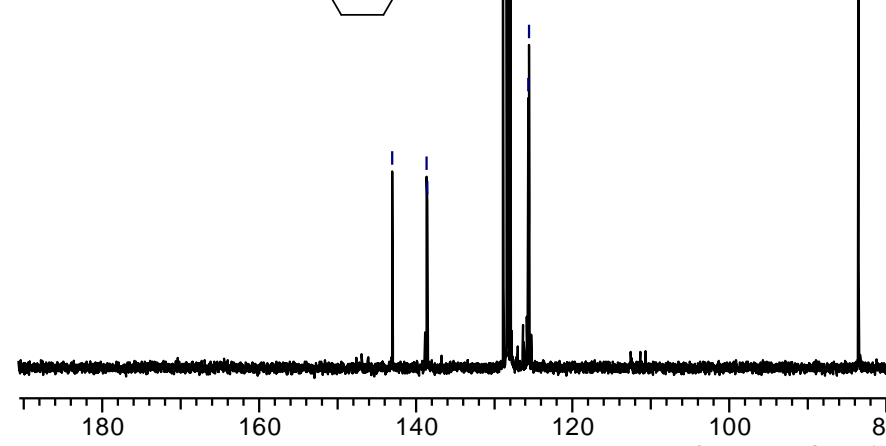
$(S, E)$-5-Methyl-1,6-diphenylhex-5-en-3-ol (10h', mixture of $(E)$ and $(Z)$ isomers) ${ }^{1} \mathbf{H}$ NMR (500 MHz, $\left.\mathrm{CDCl}_{3}\right)$ :

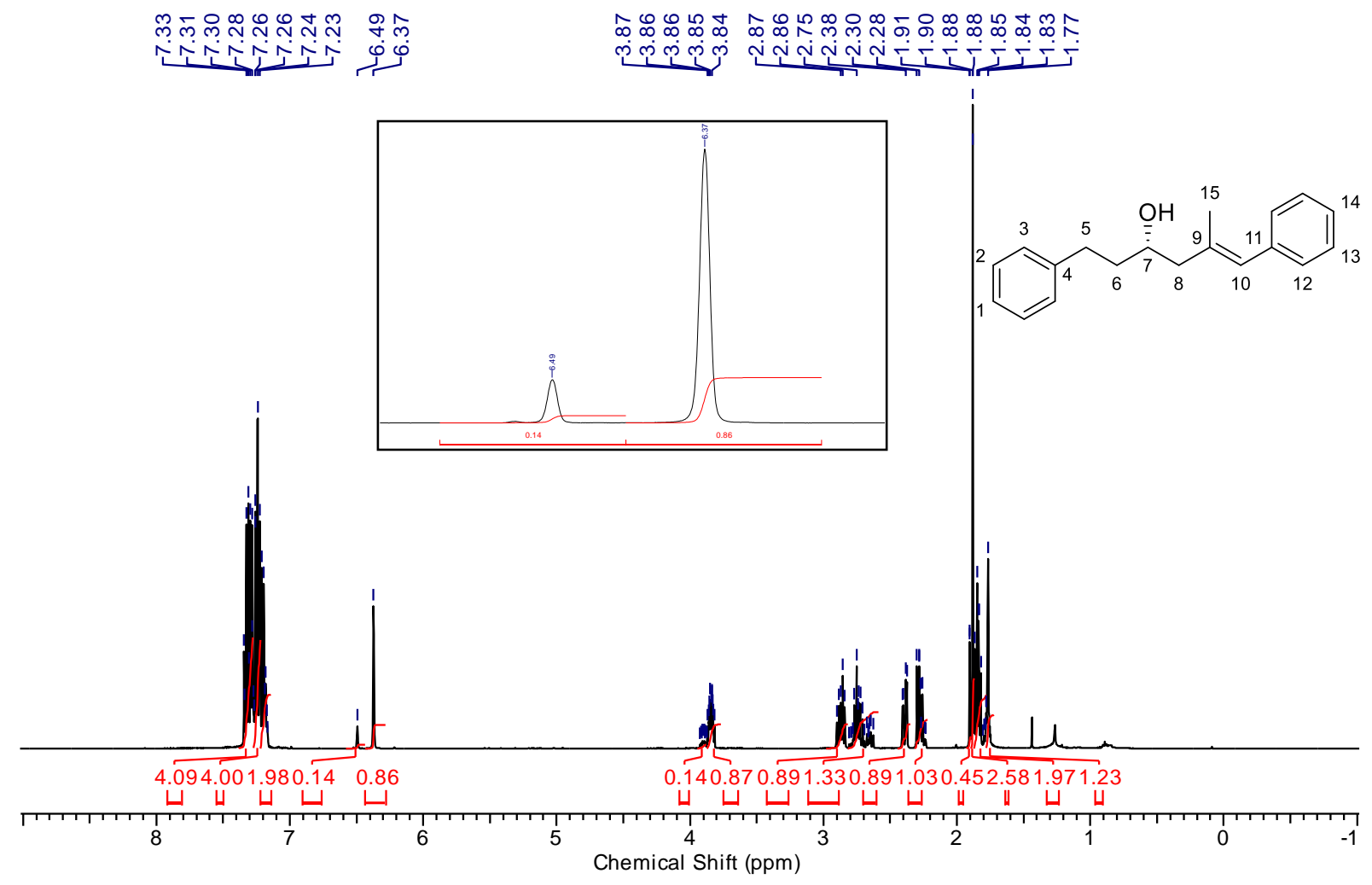

${ }^{13} \mathrm{C}$ NMR (125 MHz, $\left.\mathrm{CDCl}_{3}\right)$ :

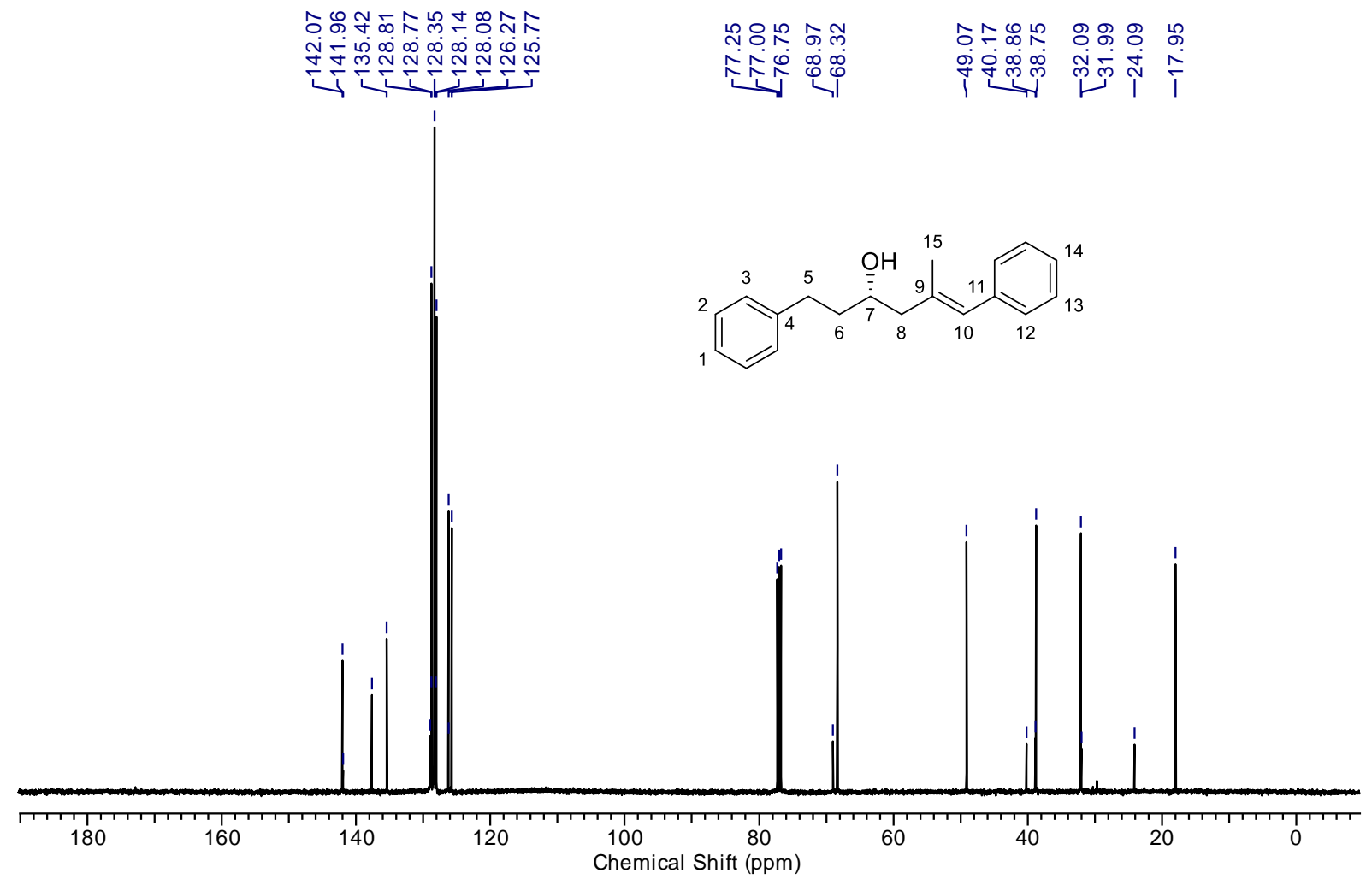


(4S,5S)-2-[(R)-1-Chloroethyl]-4,5-dicyclohexyl-1,3,2-dioxaborolane (11, crude product) ${ }^{1} \mathbf{H}$ NMR $\left(500 \mathrm{MHz}, \mathrm{CDCl}_{3}\right)$ :

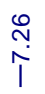

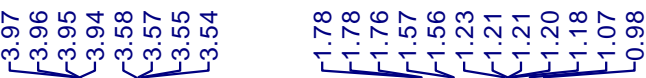
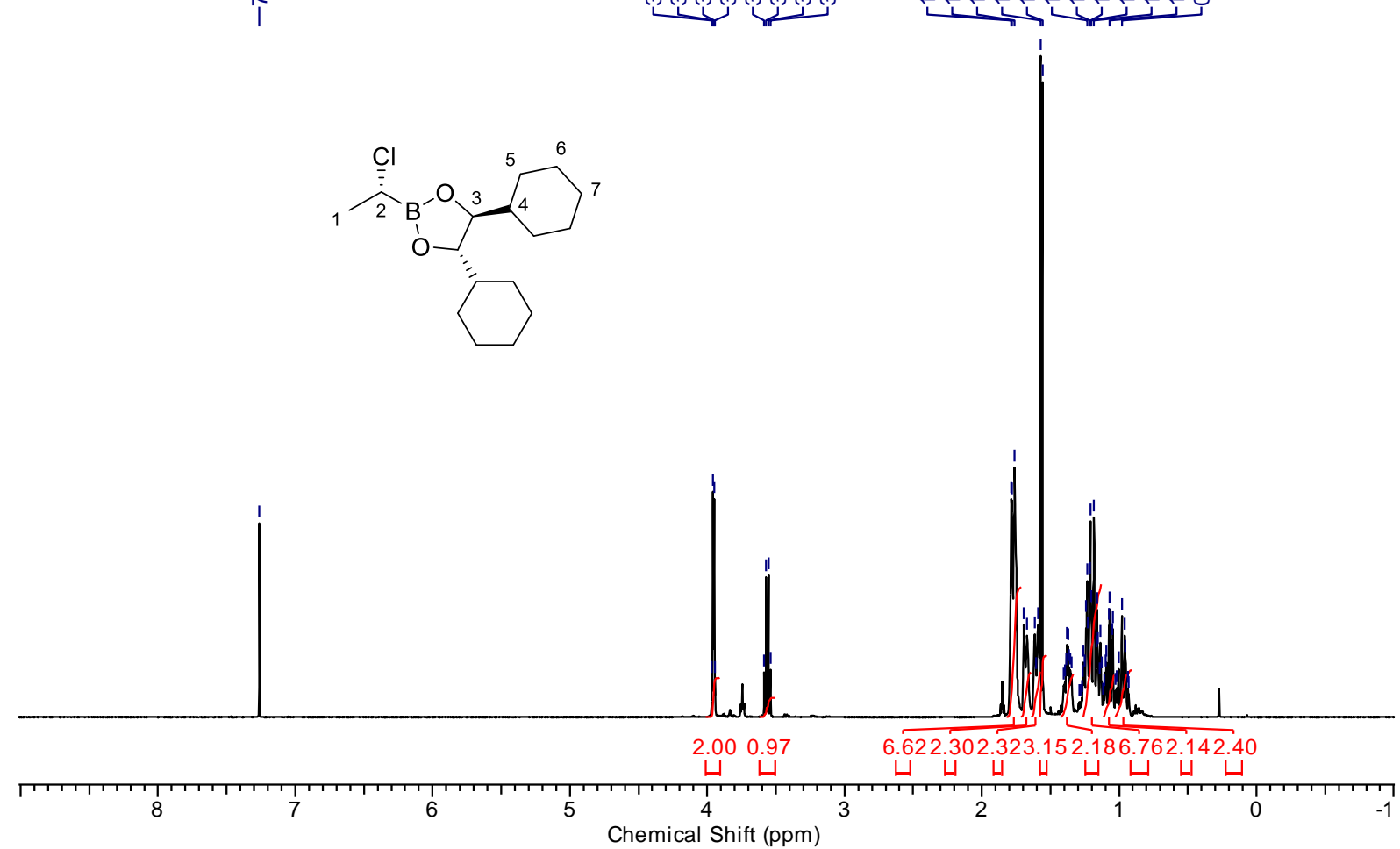

${ }^{13} \mathbf{C}$ NMR (125 MHz, $\left.\mathrm{CDCl}_{3}\right)$ :

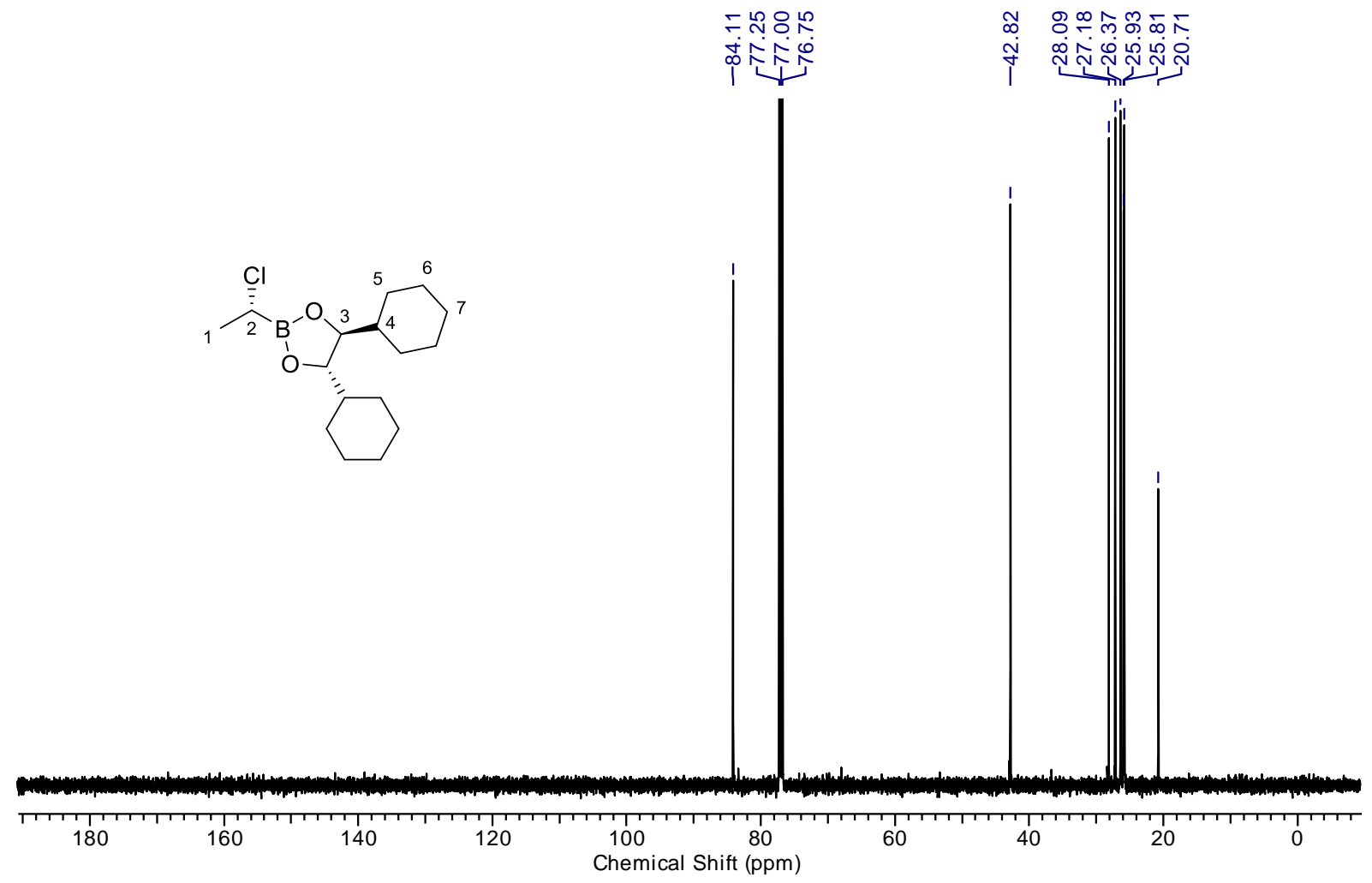


$(4 S, 5 S)-2-[(R)-C h l o r o(c y c l o h e x y l)$ methyl]-4,5-dicyclohexyl-1,3,2-dioxaborolane (12, crude product)

${ }^{1} \mathbf{H}$ NMR (400 MHz, $\left.\mathrm{CDCl}_{3}\right)$ :

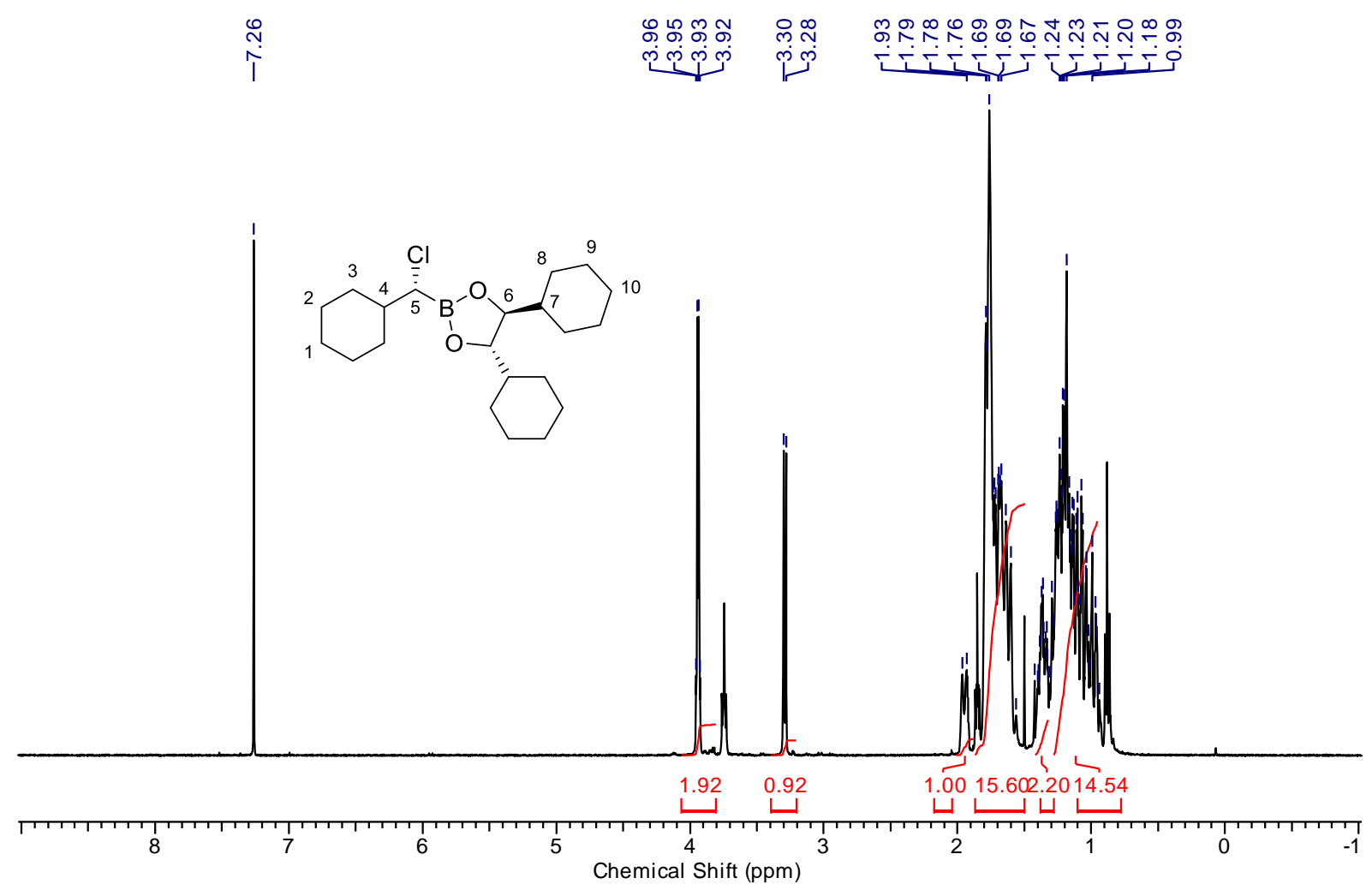

${ }^{13} \mathrm{C}$ NMR (100 MHz, $\left.\mathrm{CDCl}_{3}\right)$ :

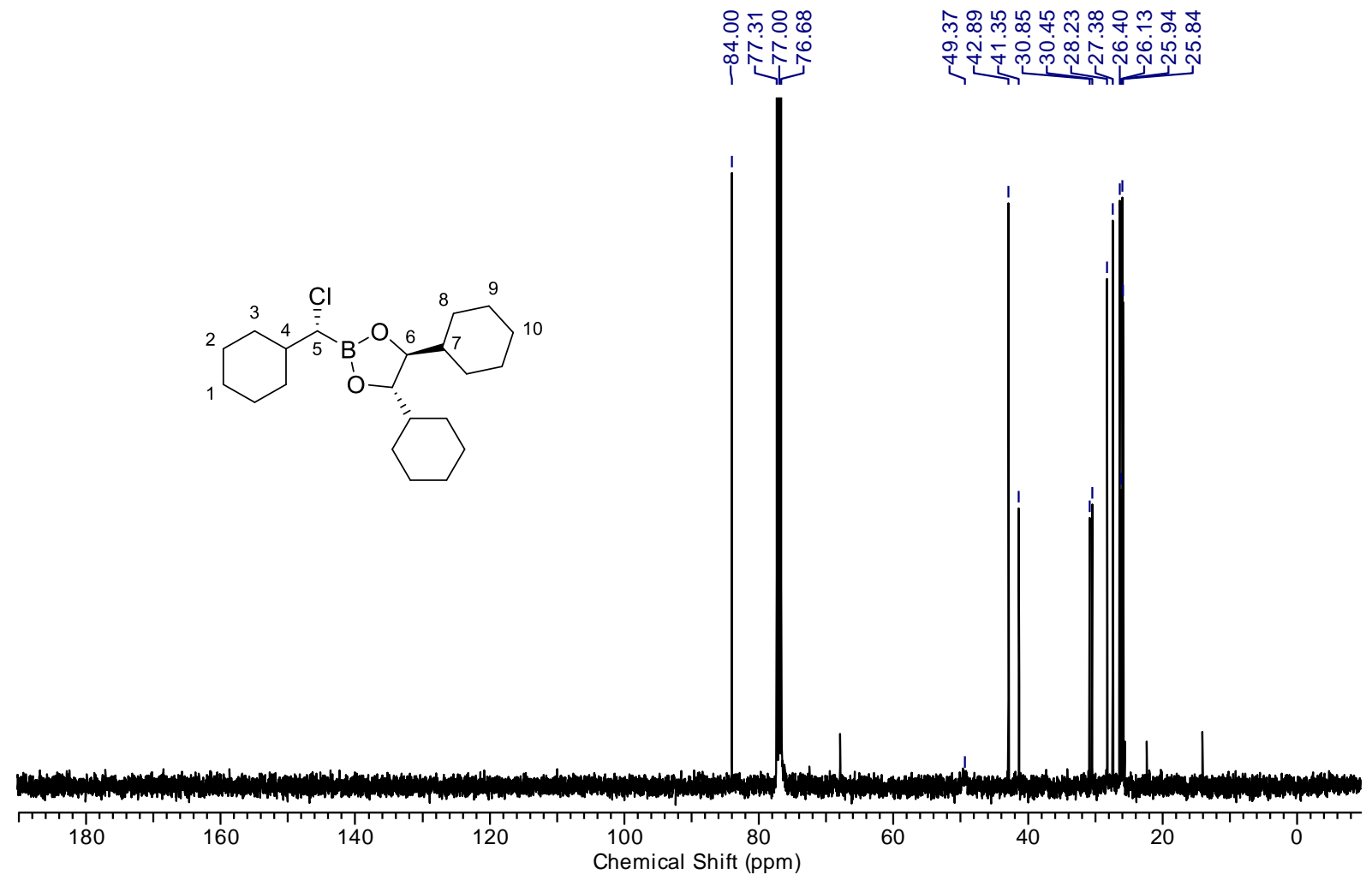




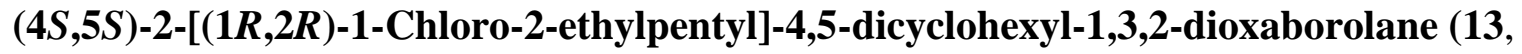
crude product)

${ }^{1} \mathbf{H}$ NMR (400 MHz, $\left.\mathrm{CDCl}_{3}\right)$ :

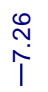
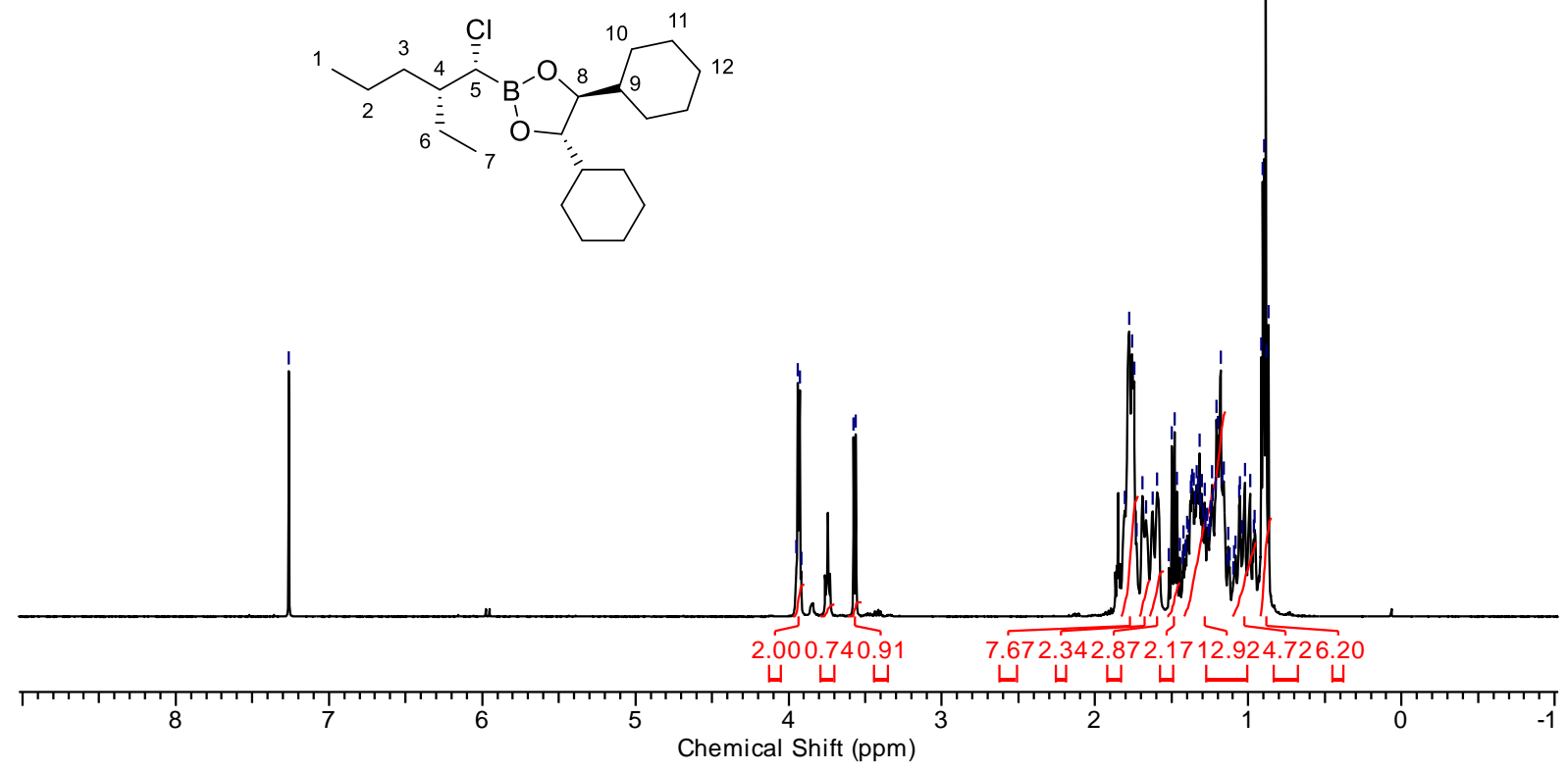

${ }^{13}$ C NMR (100 MHz, $\left.\mathrm{CDCl}_{3}\right)$ :

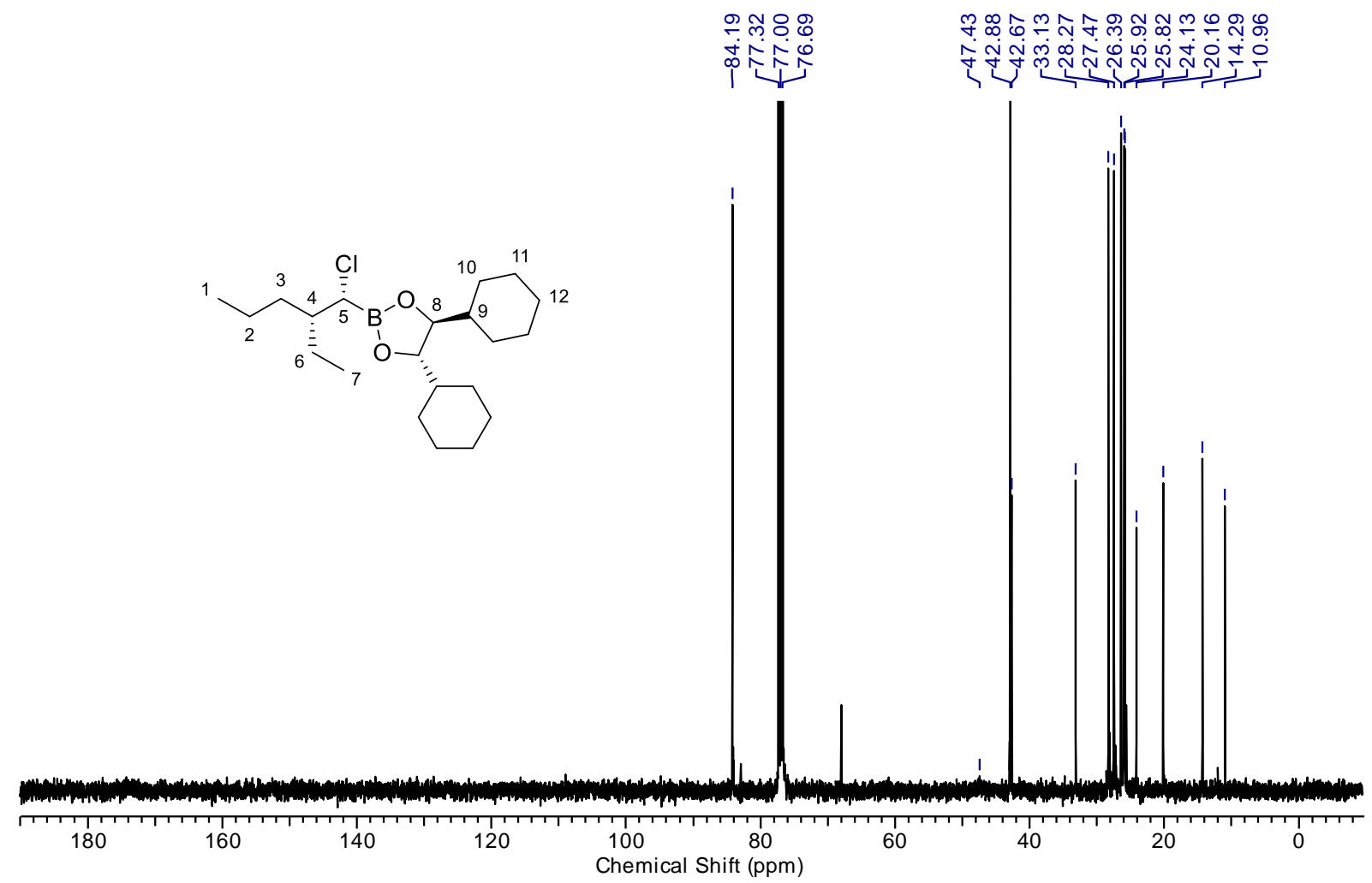


$(4 S, 5 S)-4,5-D i c y c l o h e x y l-2-[(S, Z)$-hex-4-en-2-yl]-1,3,2-dioxaborolane (14c, mixture of $(E)$ and $(Z)$ isomers)

${ }^{1} \mathbf{H}$ NMR $\left(500 \mathrm{MHz}, \mathrm{CDCl}_{3}\right)$ :

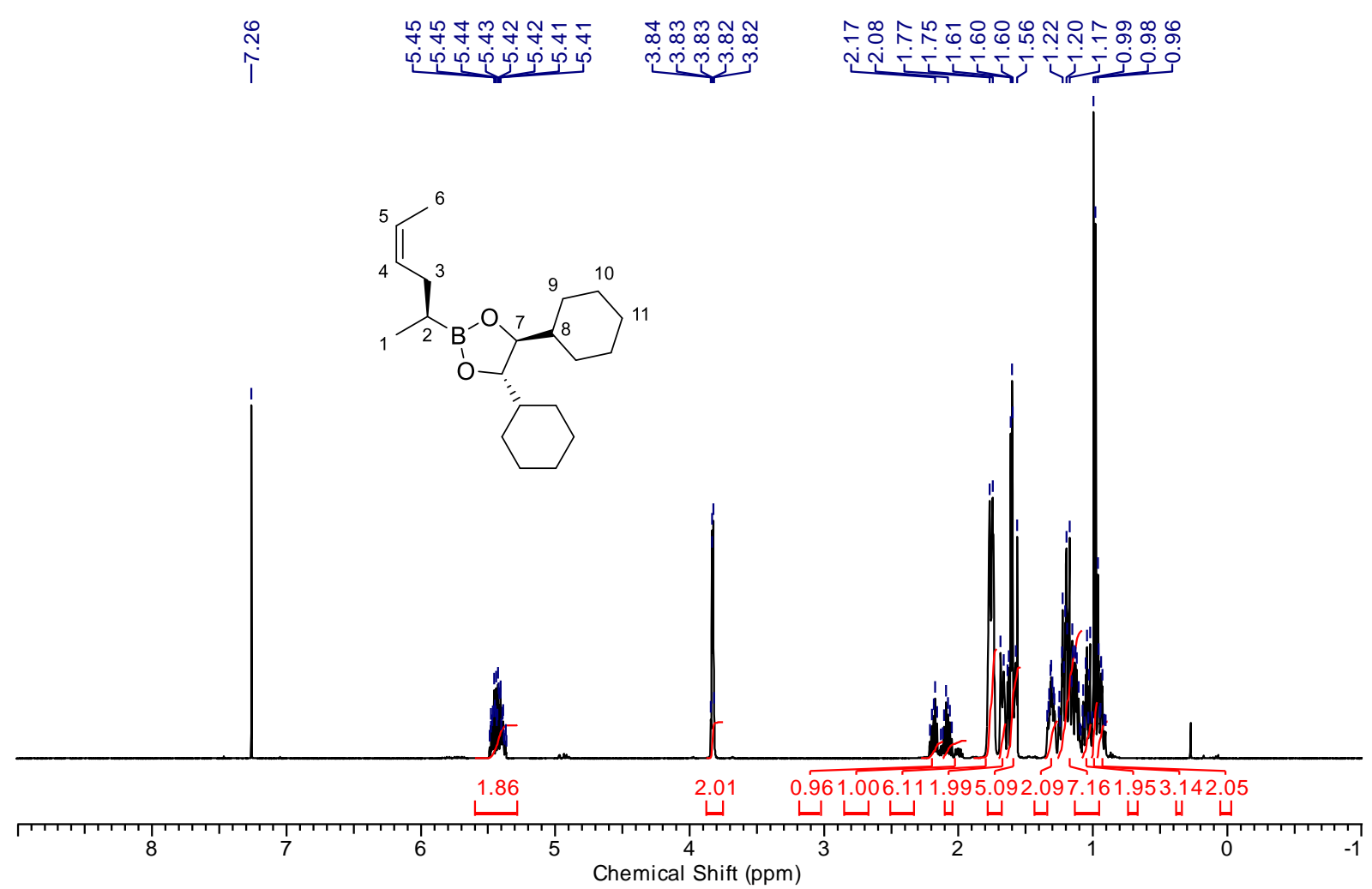

${ }^{13} \mathrm{C}$ NMR $\left(125 \mathrm{MHz}, \mathrm{CDCl}_{3}\right)$ :

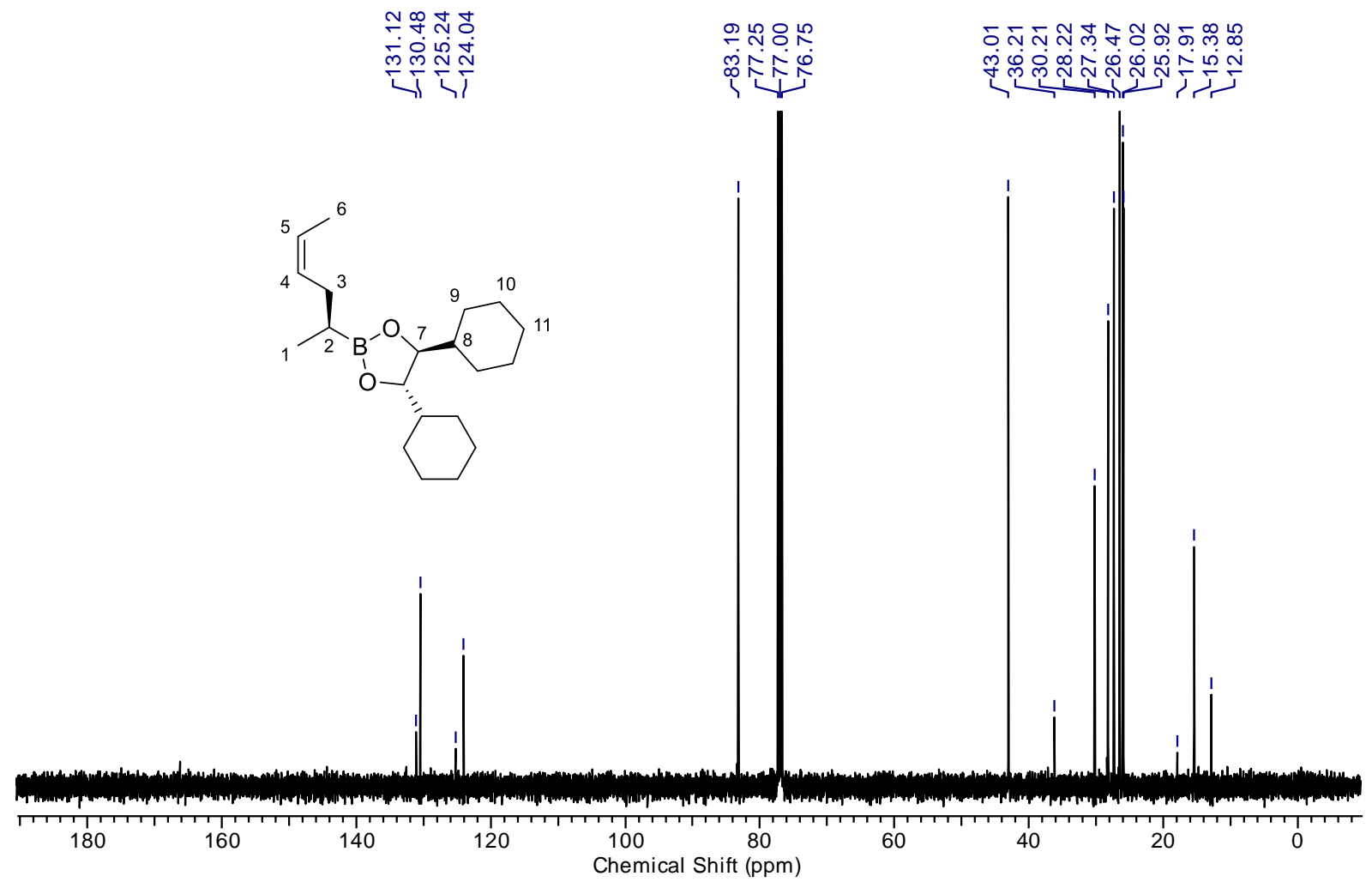


(4S,5S)-4,5-Dicyclohexyl-2-[(S,Z)-dec-4-en-2-yl]-1,3,2-dioxaborolane (14d, mixture of $(E)$ and $(Z)$ isomers)

${ }^{1} \mathbf{H}$ NMR $\left(500 \mathrm{MHz}, \mathrm{CDCl}_{3}\right)$ :

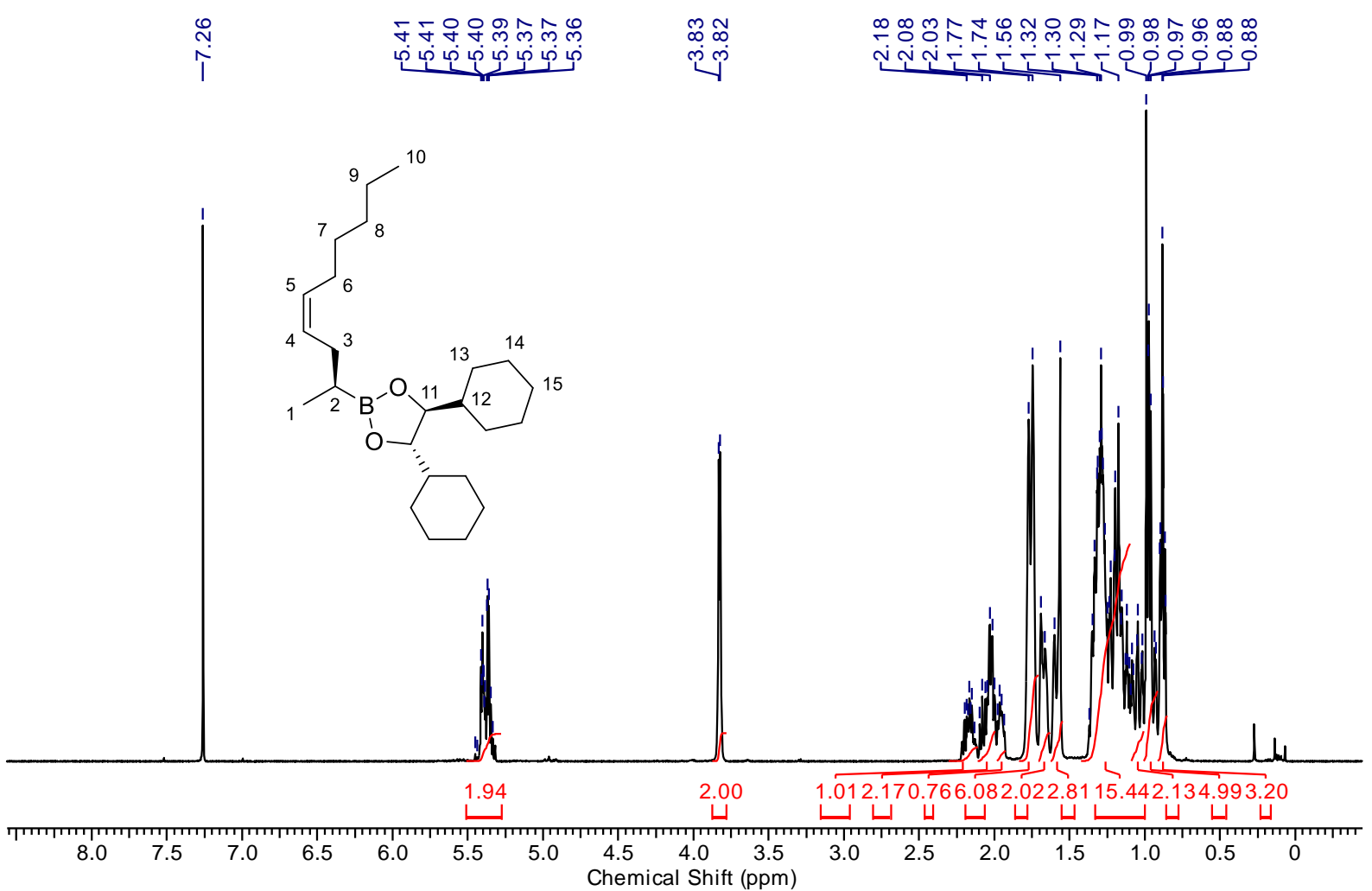

${ }^{13} \mathrm{C}$ NMR (125 MHz, $\left.\mathrm{CDCl}_{3}\right)$ :

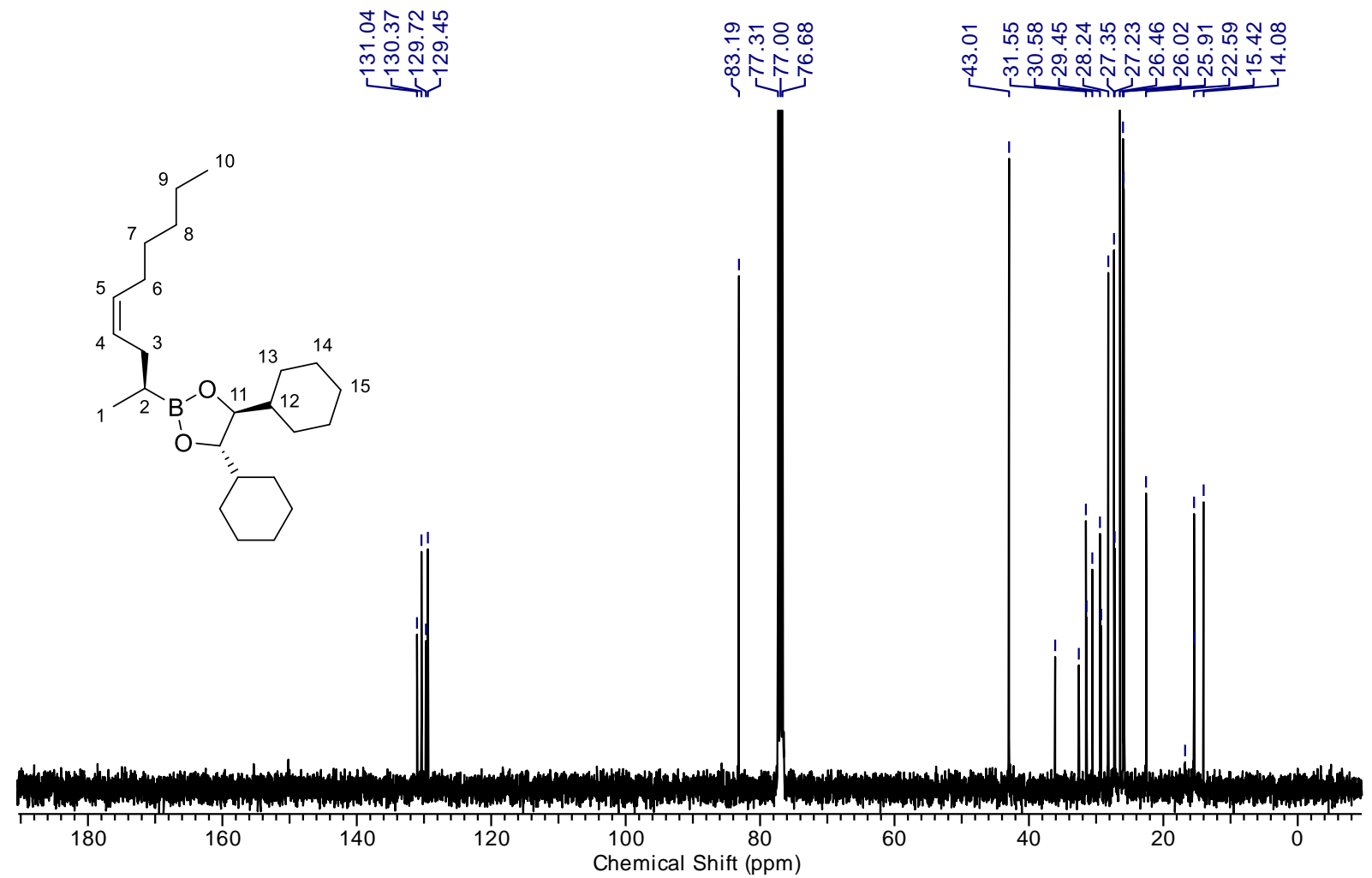



mixture of $(E)$ and $(Z)$ isomers)

${ }^{1} \mathbf{H}$ NMR $\left(500 \mathrm{MHz}, \mathrm{CDCl}_{3}\right)$ :

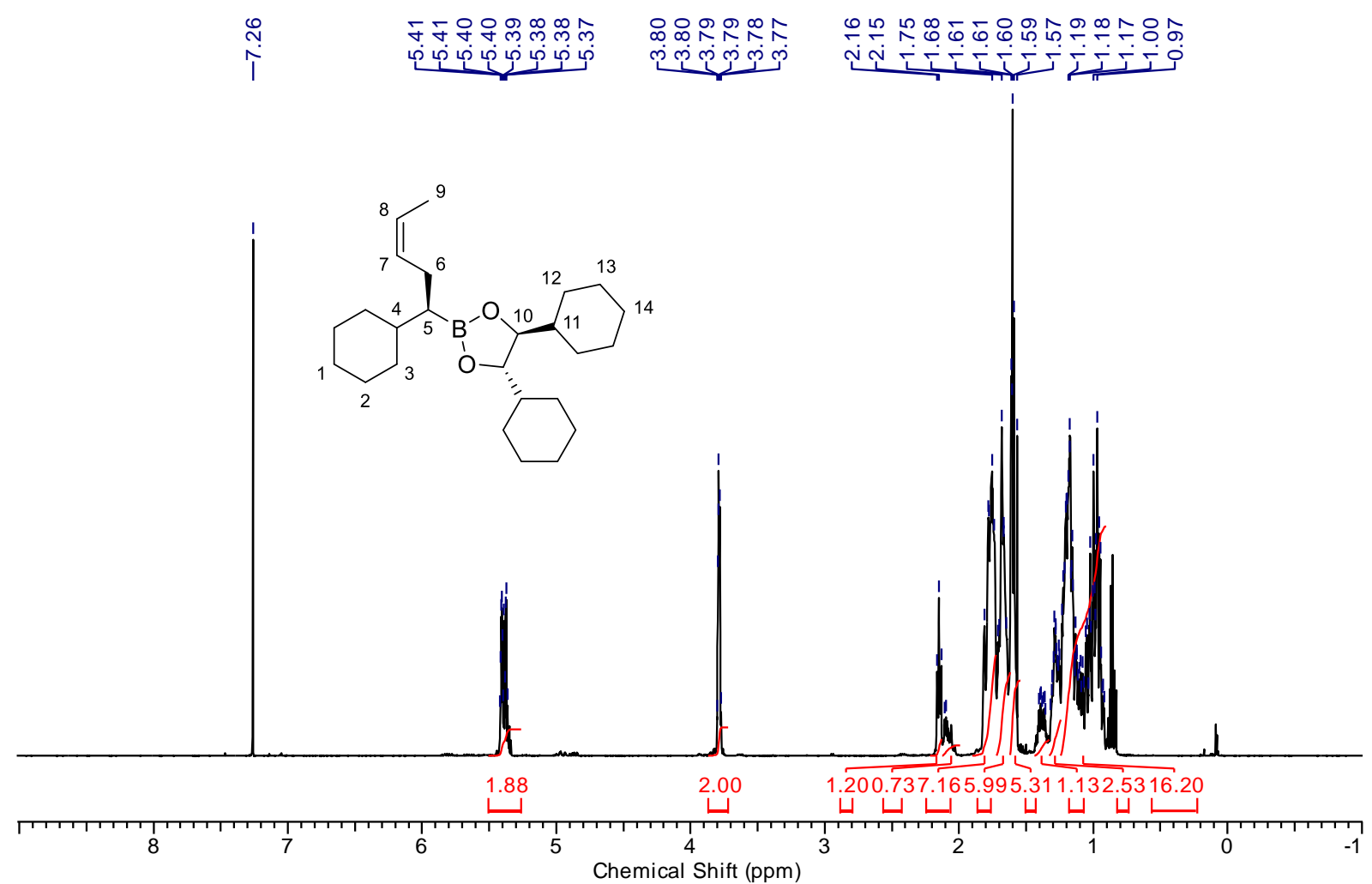

${ }^{13}$ C NMR (125 MHz, $\left.\mathrm{CDCl}_{3}\right)$ :

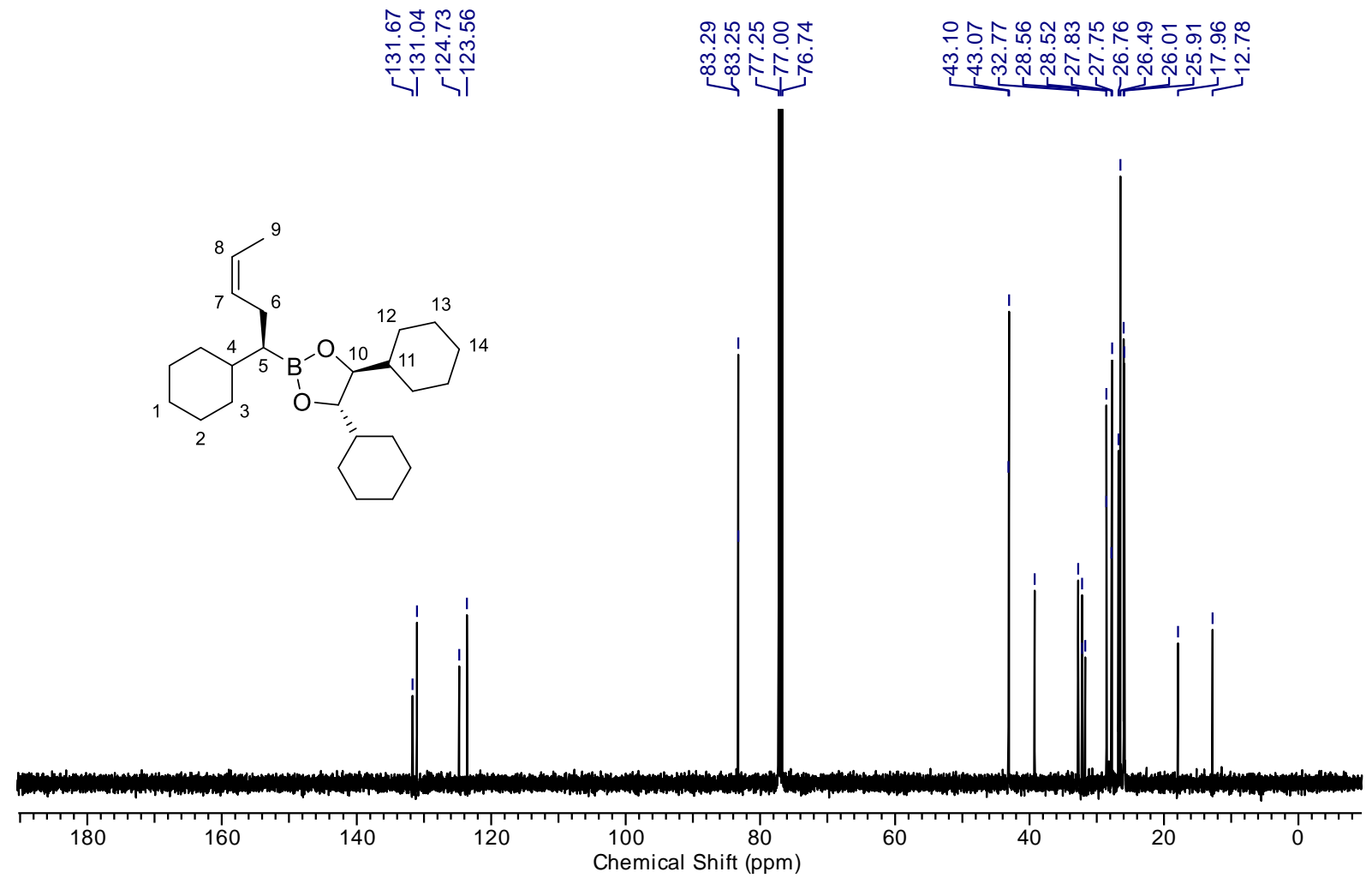


(4S,5S)-4,5-Dicyclohexyl-2-[(R,E)-1-cyclohexylnon-3-en-1-yl]-1,3,2-dioxaborolane $\quad(15 d$, mixture of $(E)$ and $(Z)$ isomers)

${ }^{1} \mathbf{H}$ NMR (400 MHz, $\left.\mathrm{CDCl}_{3}\right)$ :

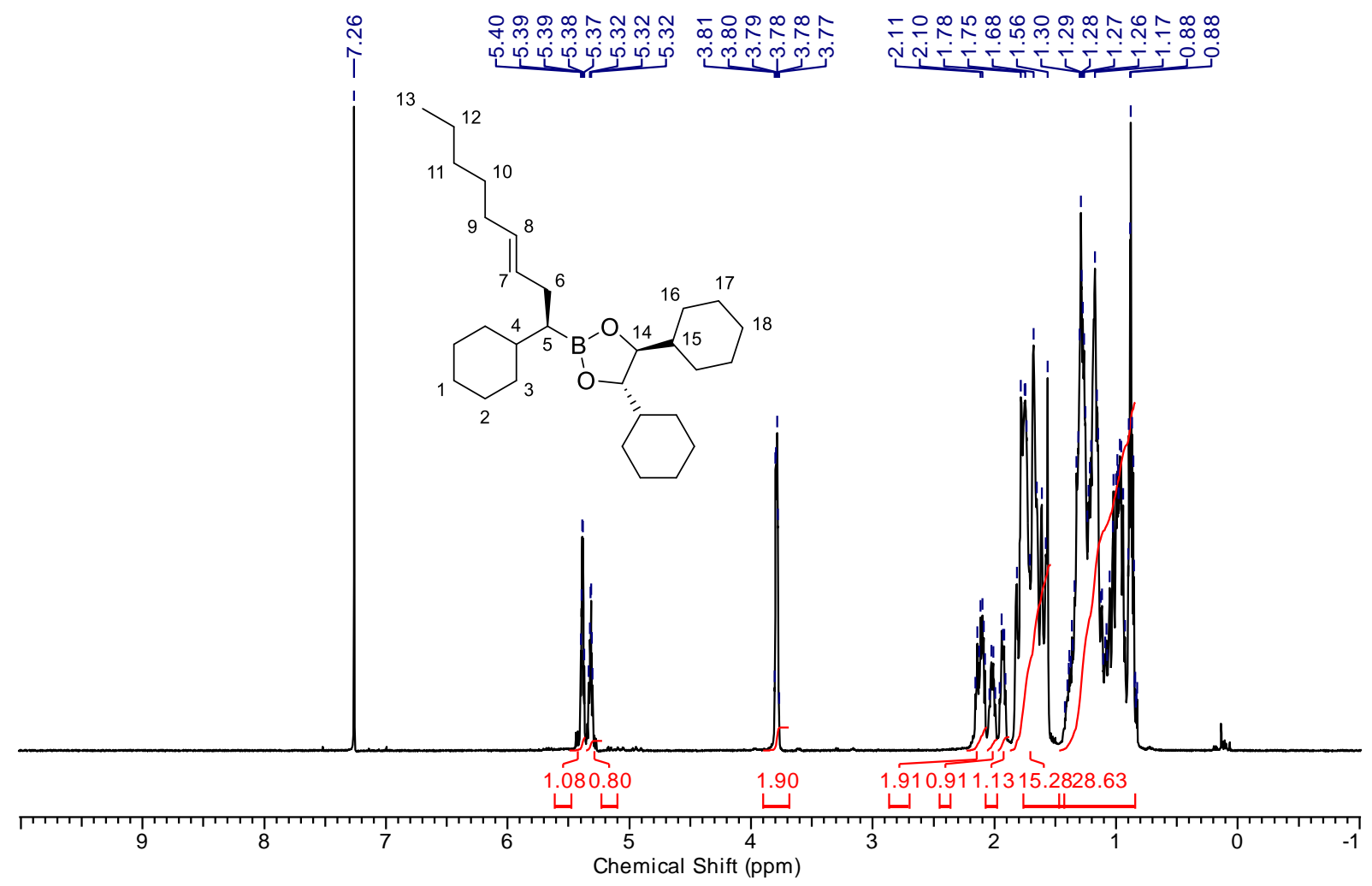

${ }^{13}$ C NMR (100 MHz, $\left.\mathrm{CDCl}_{3}\right)$ :

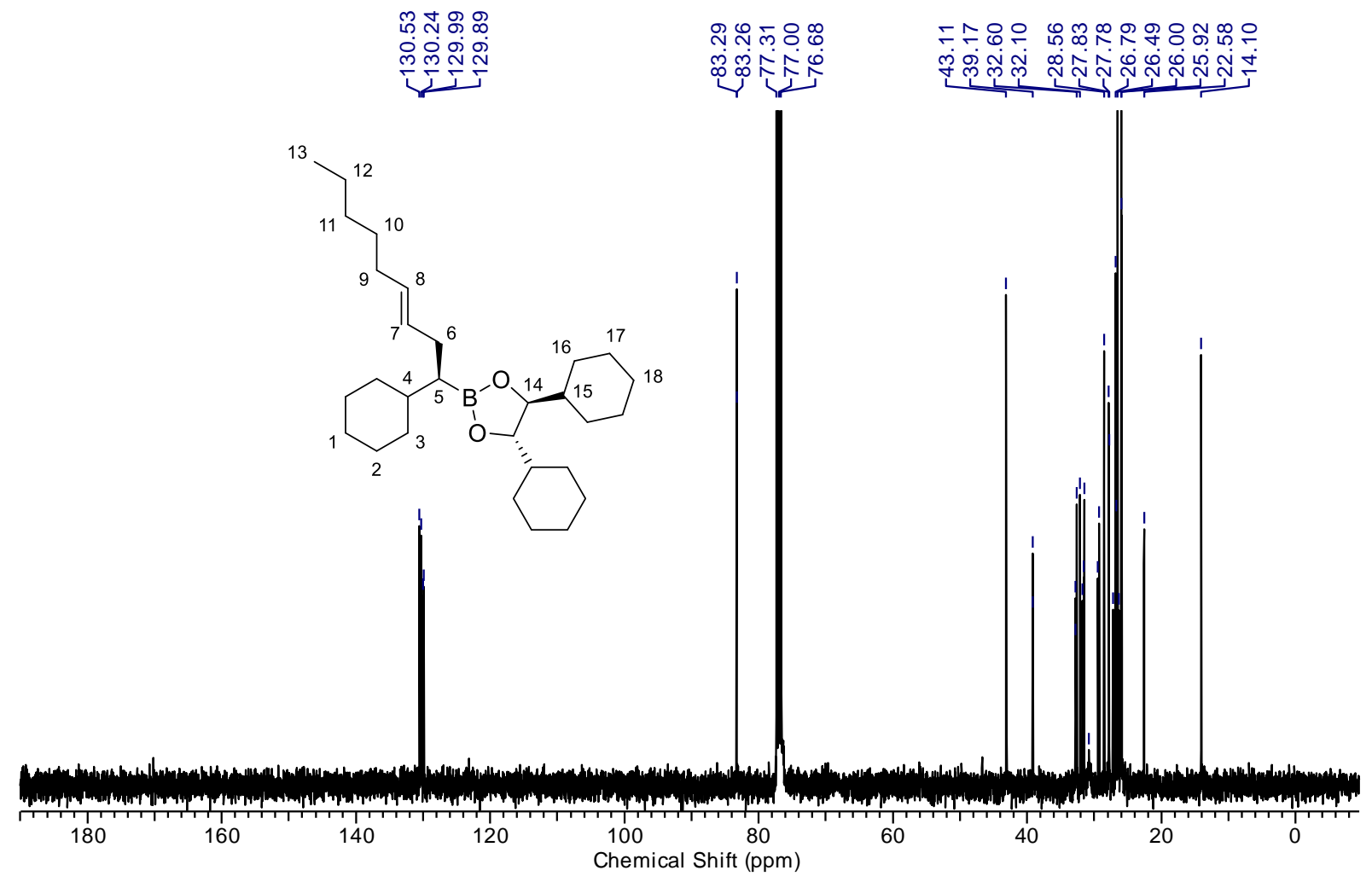


(4S,5S)-4,5-Dicyclohexyl-2-[(R,E)-1-cyclohexyl-5-methylhex-3-en-1-yl]-1,3,2dioxaborolane (15e, mixture of $(E)$ and $(Z)$ isomers)

${ }^{1} \mathbf{H}$ NMR (500 MHz, $\left.\mathrm{CDCl}_{3}\right)$ :
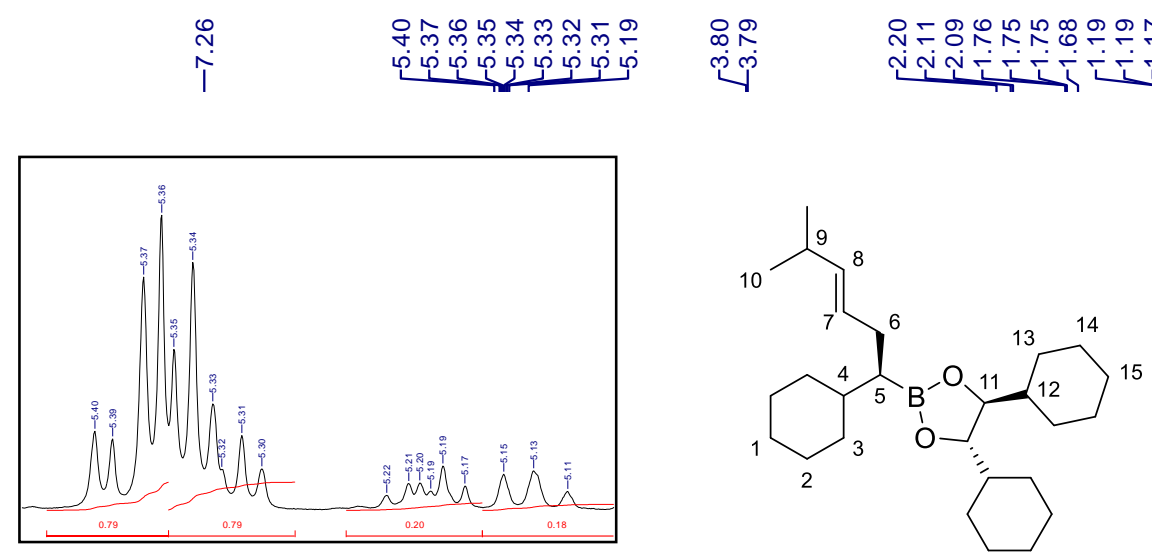

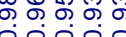

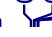

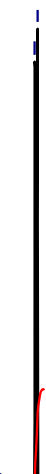

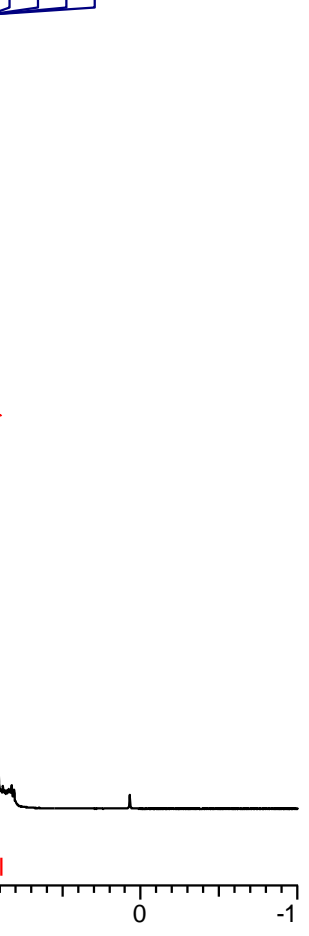

${ }^{13} \mathrm{C}$ NMR (125 MHz, $\left.\mathrm{CDCl}_{3}\right)$ :

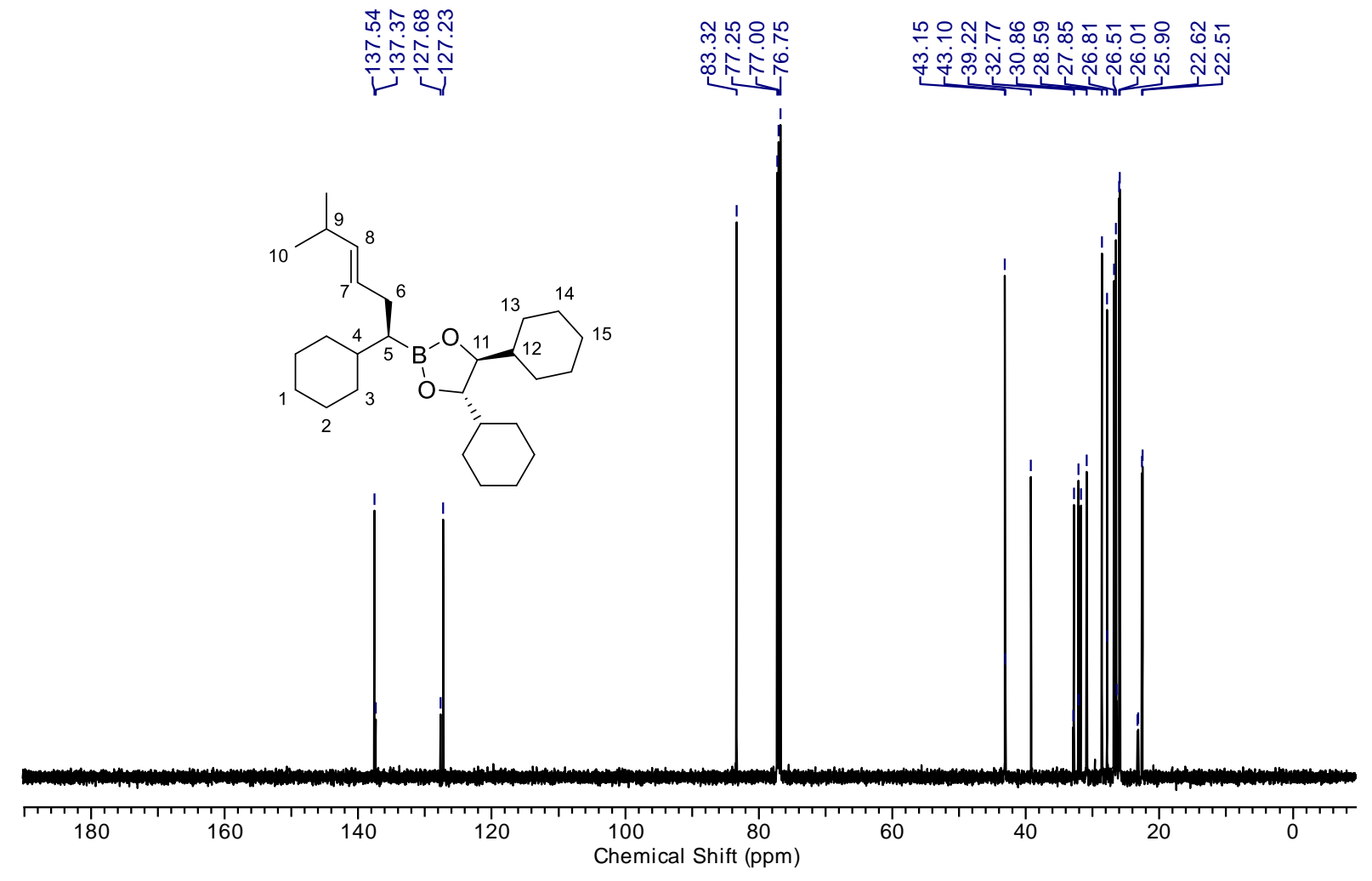



mixture of $(E)$ and $(Z)$ isomers)

${ }^{1} \mathbf{H}$ NMR (500 MHz, $\left.\mathrm{CDCl}_{3}\right)$ :

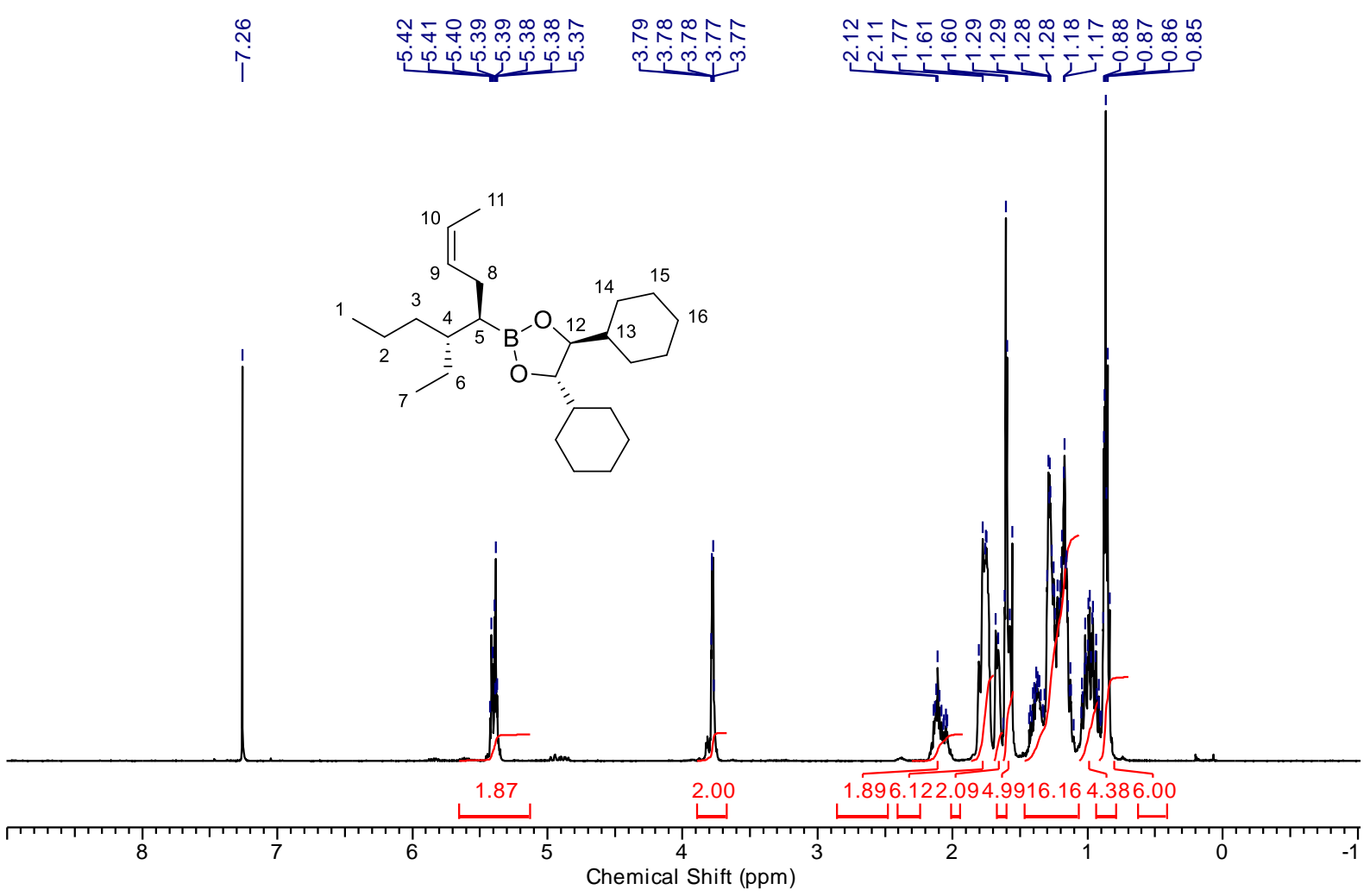

${ }^{13}$ C NMR (125 MHz, $\left.\mathrm{CDCl}_{3}\right)$ :

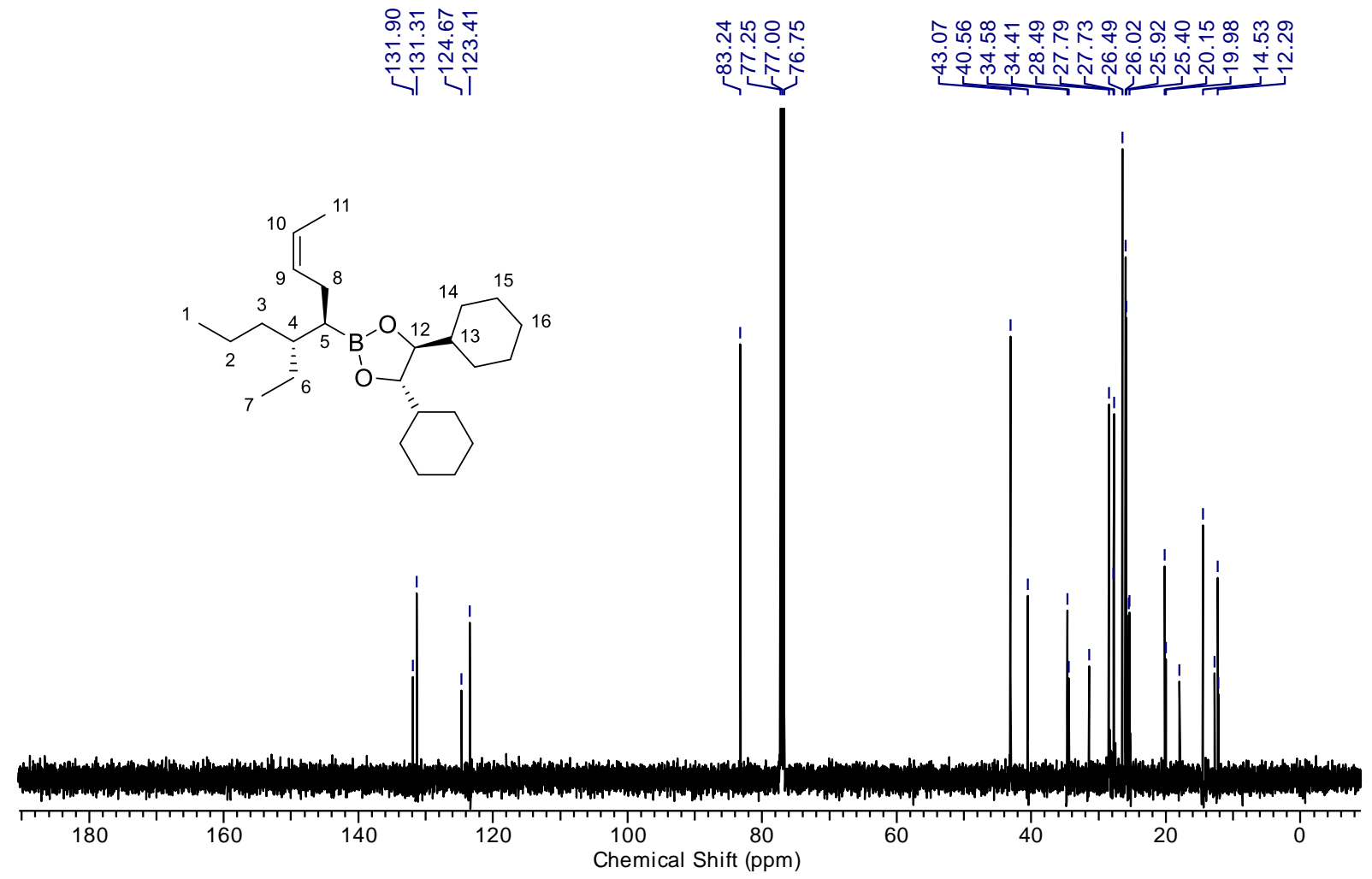



mixture of $(E)$ and $(Z)$ isomers)

${ }^{1} \mathbf{H}$ NMR $\left(500 \mathrm{MHz}, \mathrm{CDCl}_{3}\right)$ :

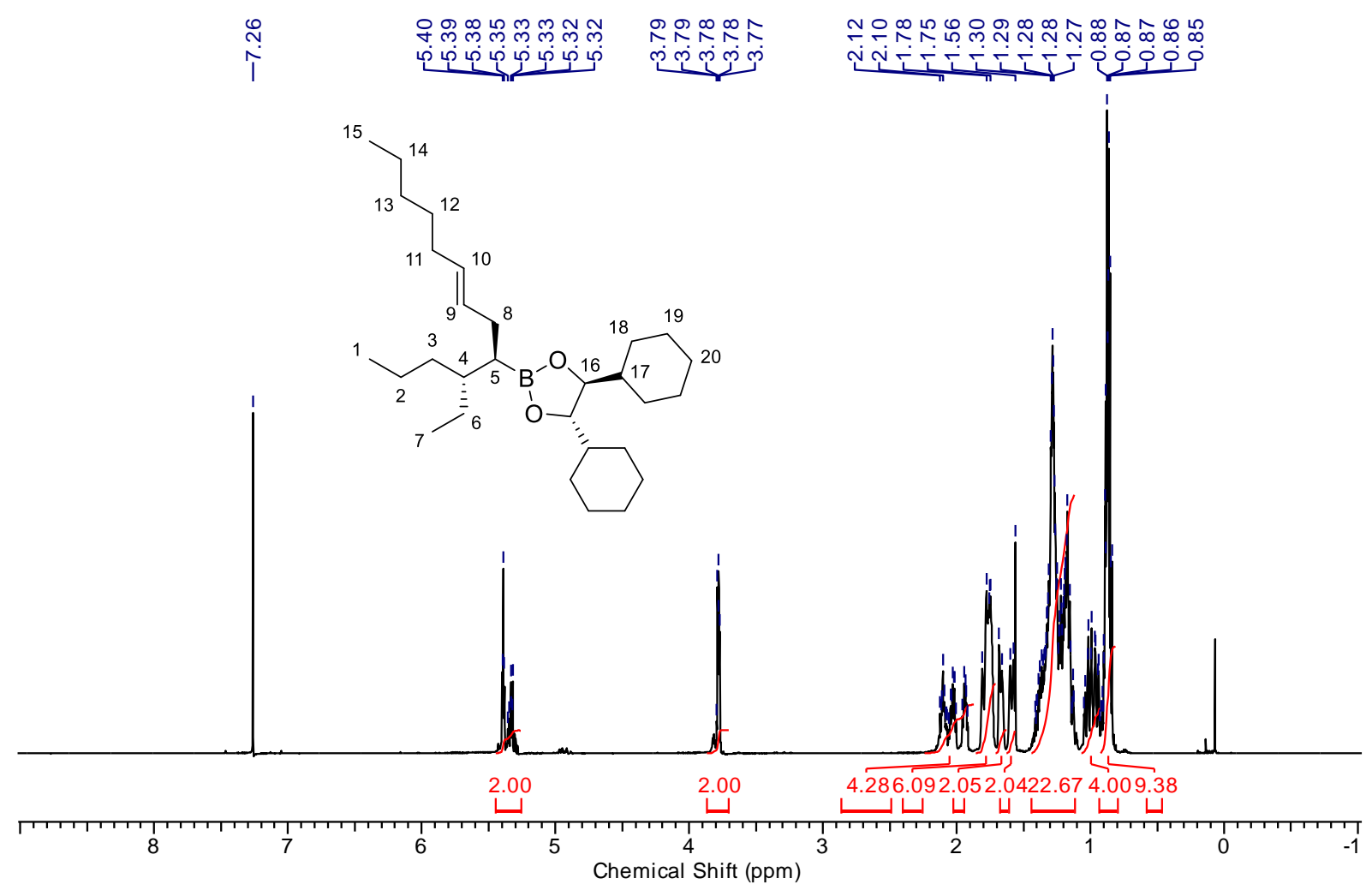

${ }^{13}$ C NMR (125 MHz, $\left.\mathrm{CDCl}_{3}\right)$ :

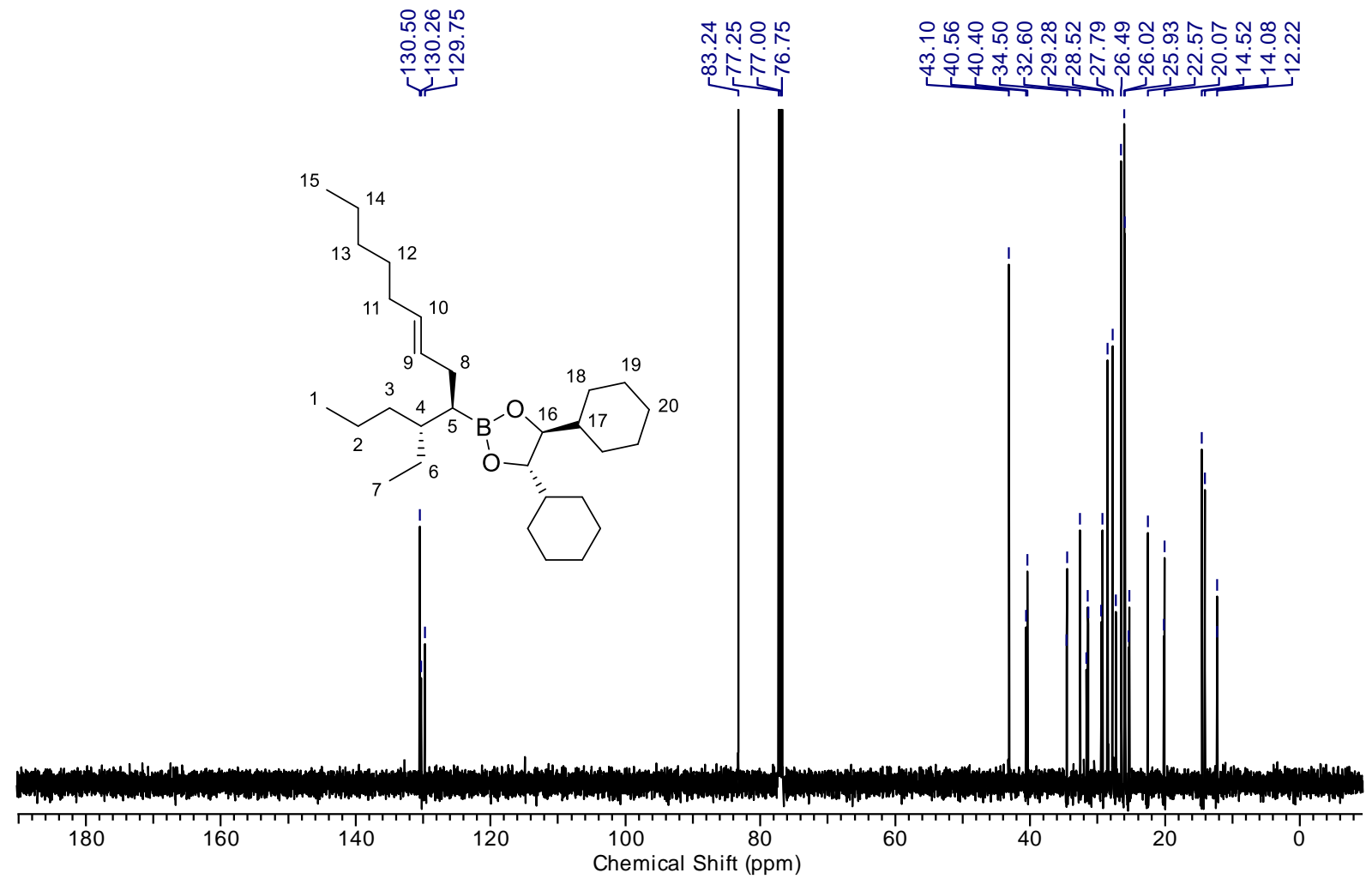


(4S,5S)-4,5-Dicyclohexyl-2-[(4R,5R,E)-4-ethyl-9-methyldec-7-en-5-yl]-1,3,2dioxaborolane (16e, mixture of $(E)$ and $(Z)$ isomers)

${ }^{1} \mathbf{H}$ NMR (500 MHz, $\left.\mathrm{CDCl}_{3}\right)$ :

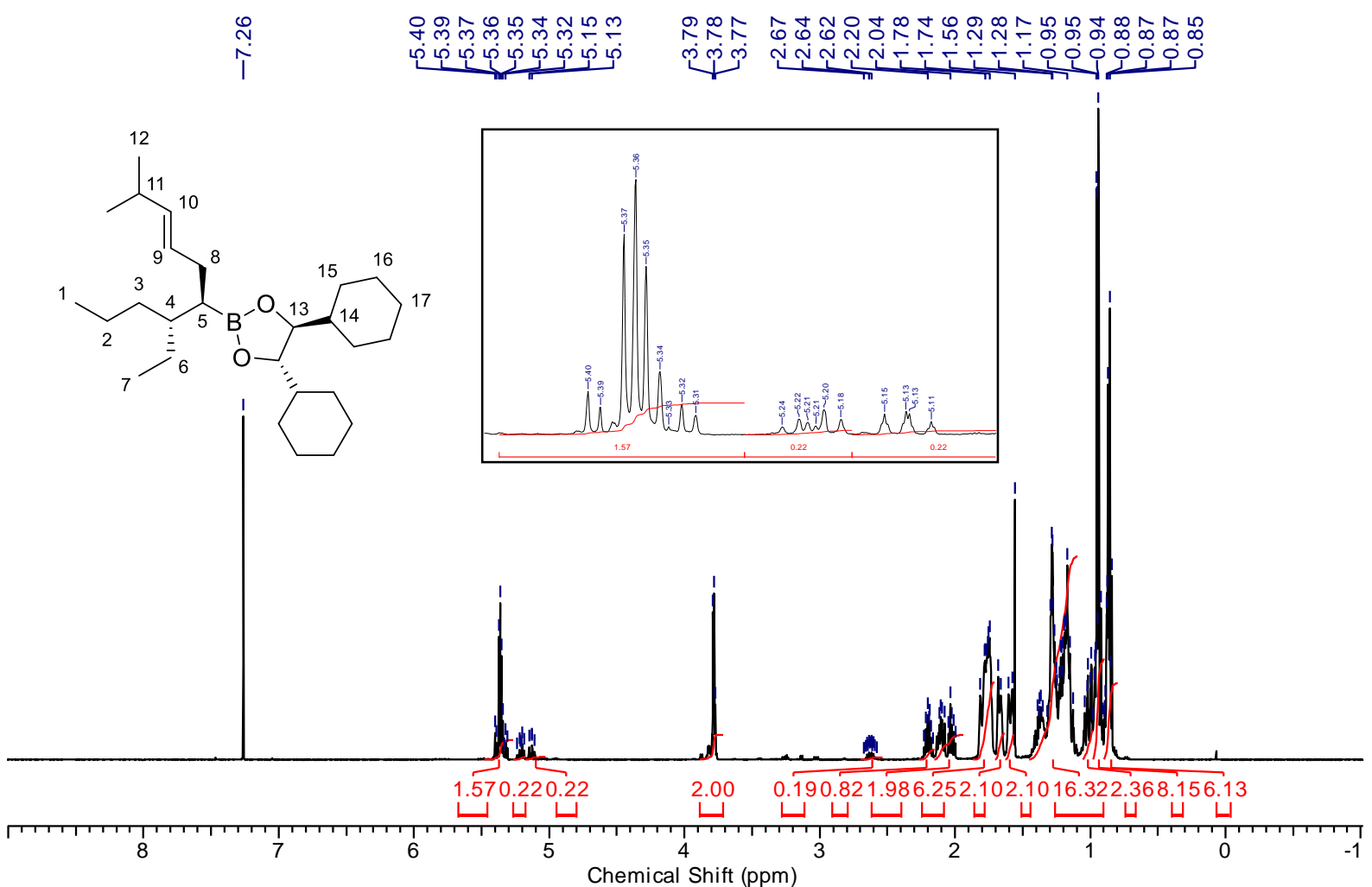

${ }^{13} \mathrm{C}$ NMR (125 MHz, $\left.\mathrm{CDCl}_{3}\right)$ :

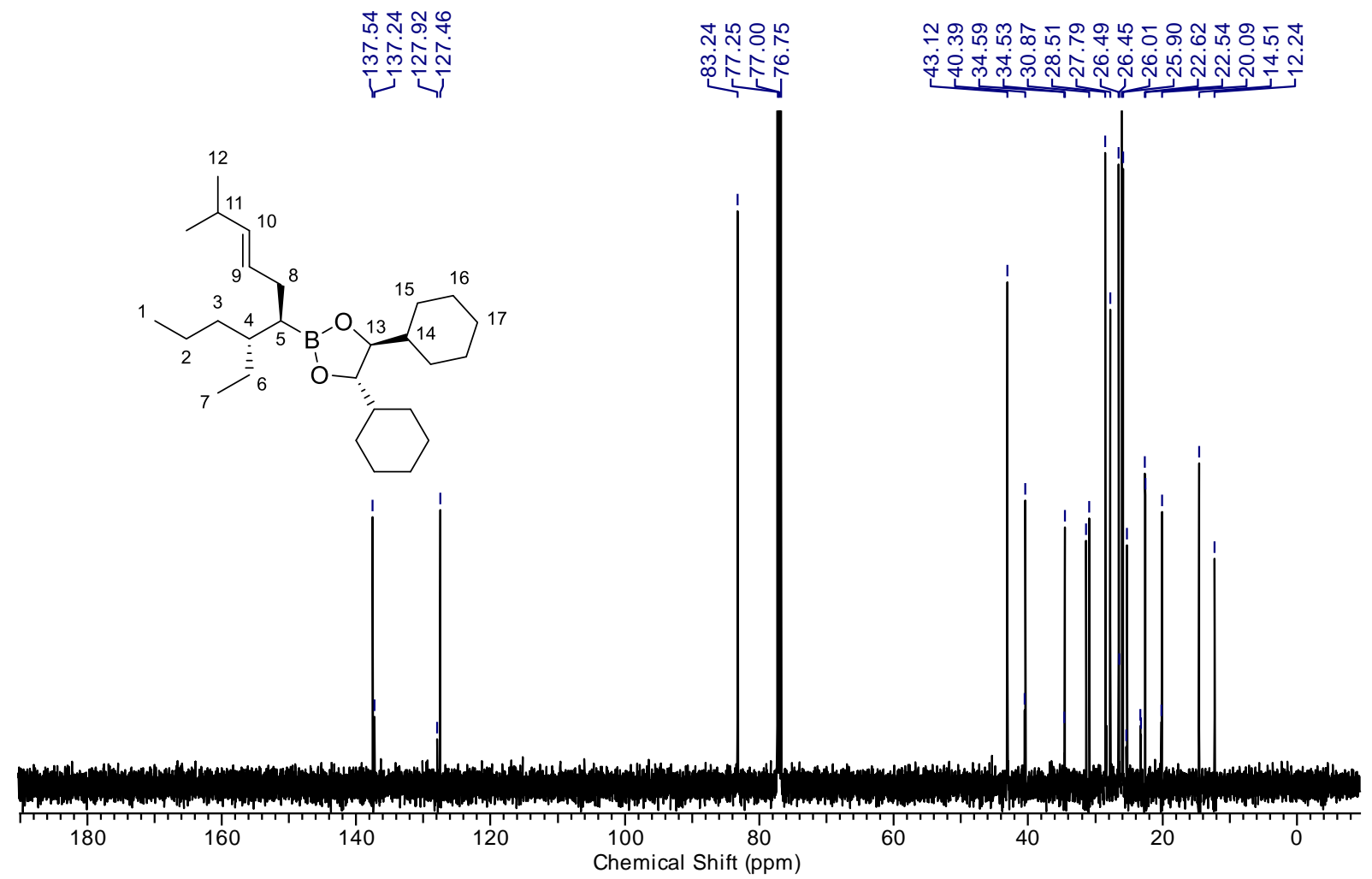


$(S, Z)$-Hex-4-en-2-ol (17c, 75 wt-\% in pentane, di ethyl ether; mixture of $(E)$ and $(Z)$ isomers) ${ }^{1} \mathbf{H}$ NMR $\left(500 \mathrm{MHz}, \mathrm{CDCl}_{3}\right)$ :
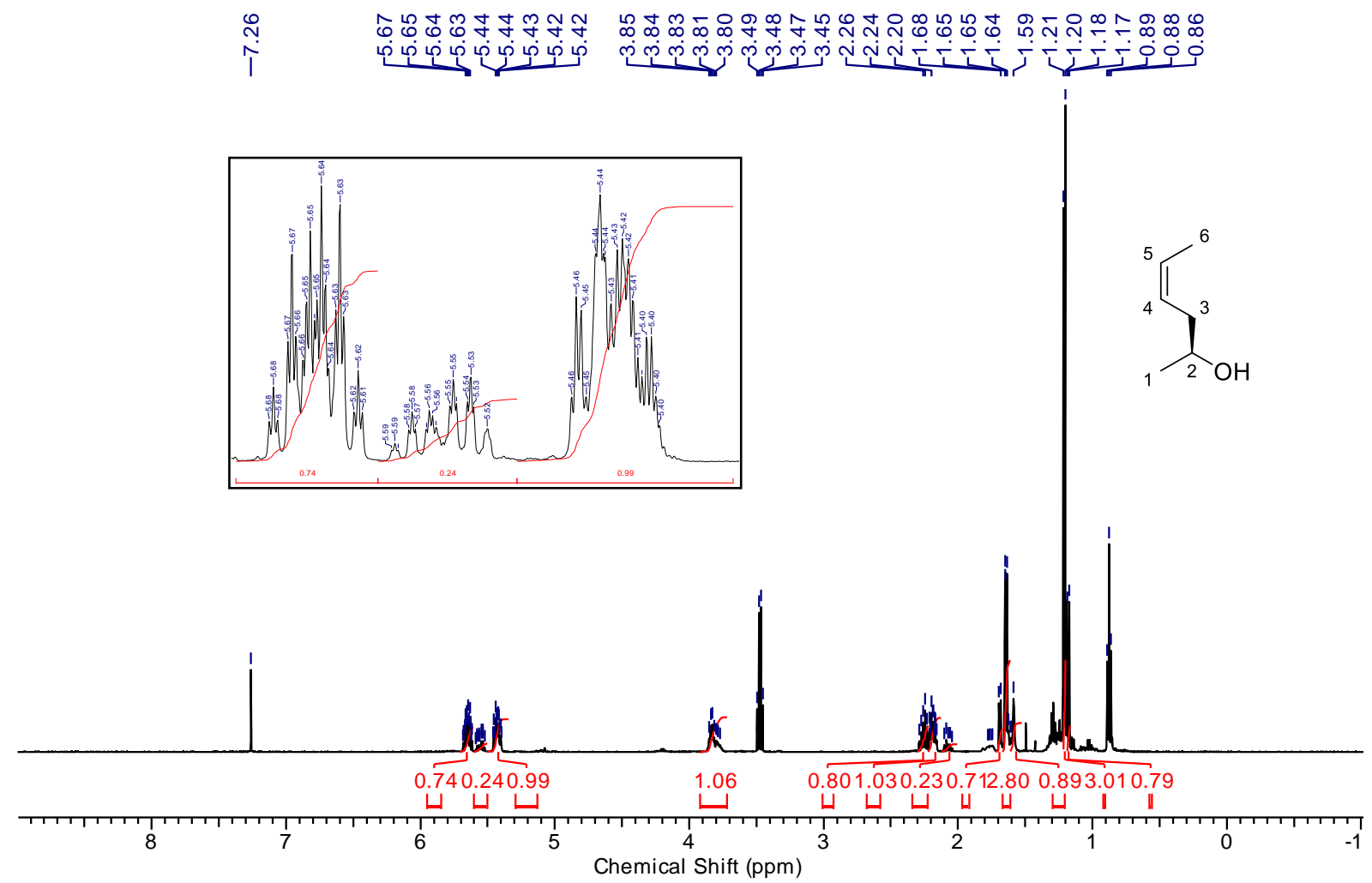

${ }^{13} \mathrm{C}$ NMR $\left(125 \mathrm{MHz}, \mathrm{CDCl}_{3}\right)$ :

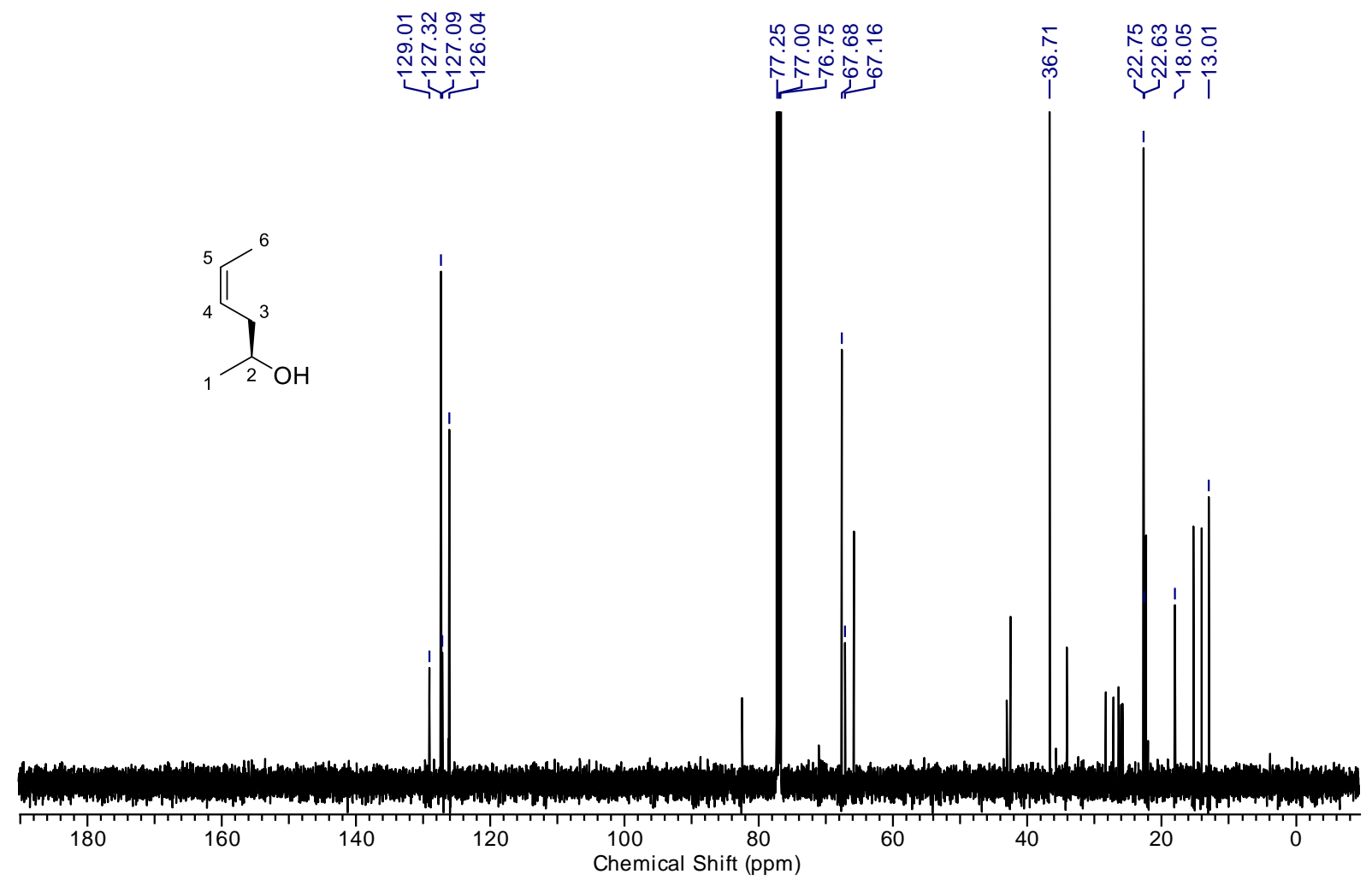


(S,Z)-Dec-4-en-2-ol (17d, mixture of $(E)$ and $(Z)$ isomers)

${ }^{1} \mathbf{H}$ NMR (500 MHz, $\left.\mathrm{CDCl}_{3}\right)$ :
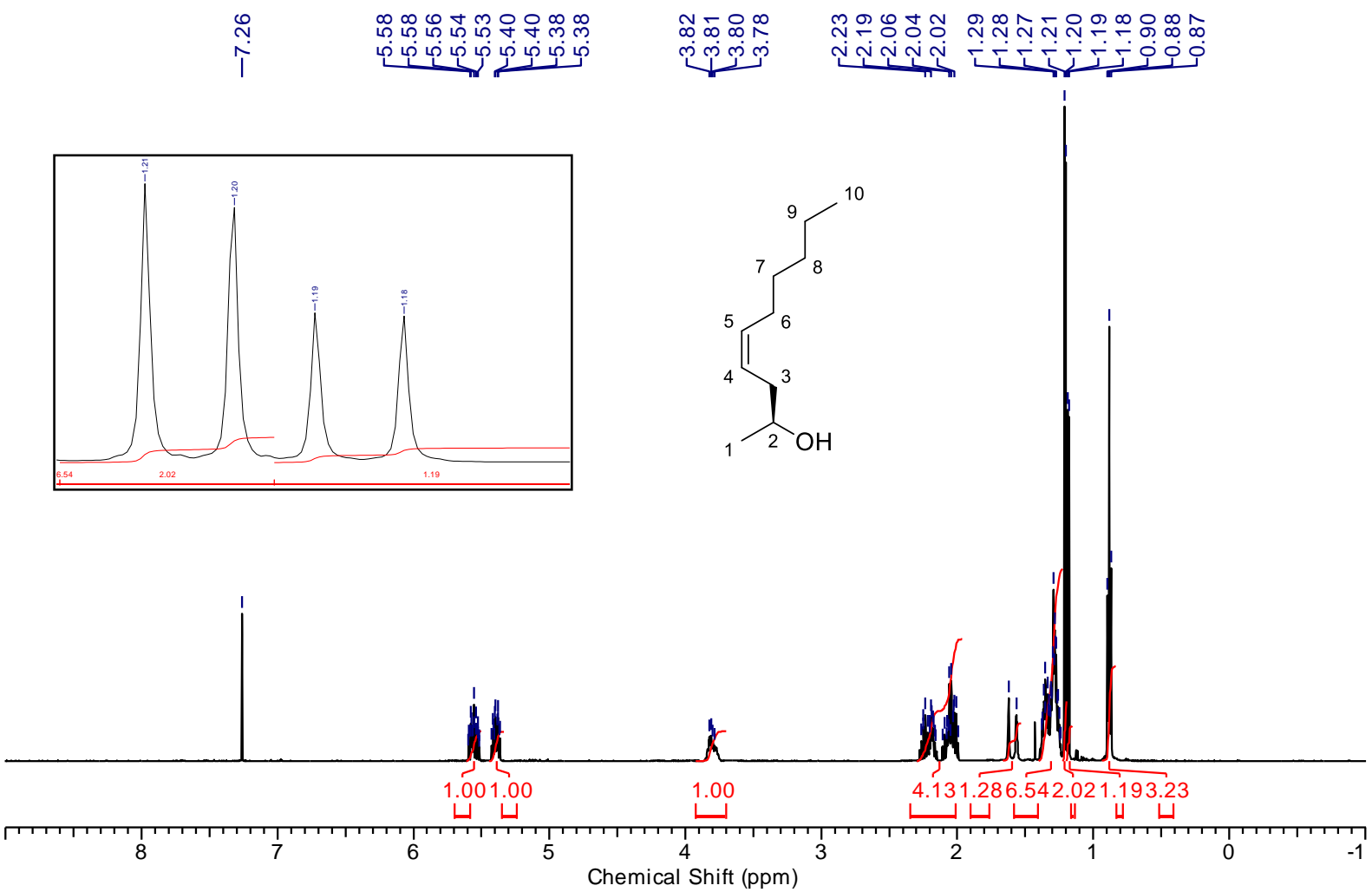

${ }^{13} \mathrm{C}$ NMR (125 MHz, $\left.\mathrm{CDCl}_{3}\right)$ :

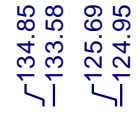

究碎

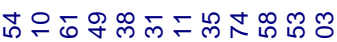

소응

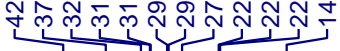

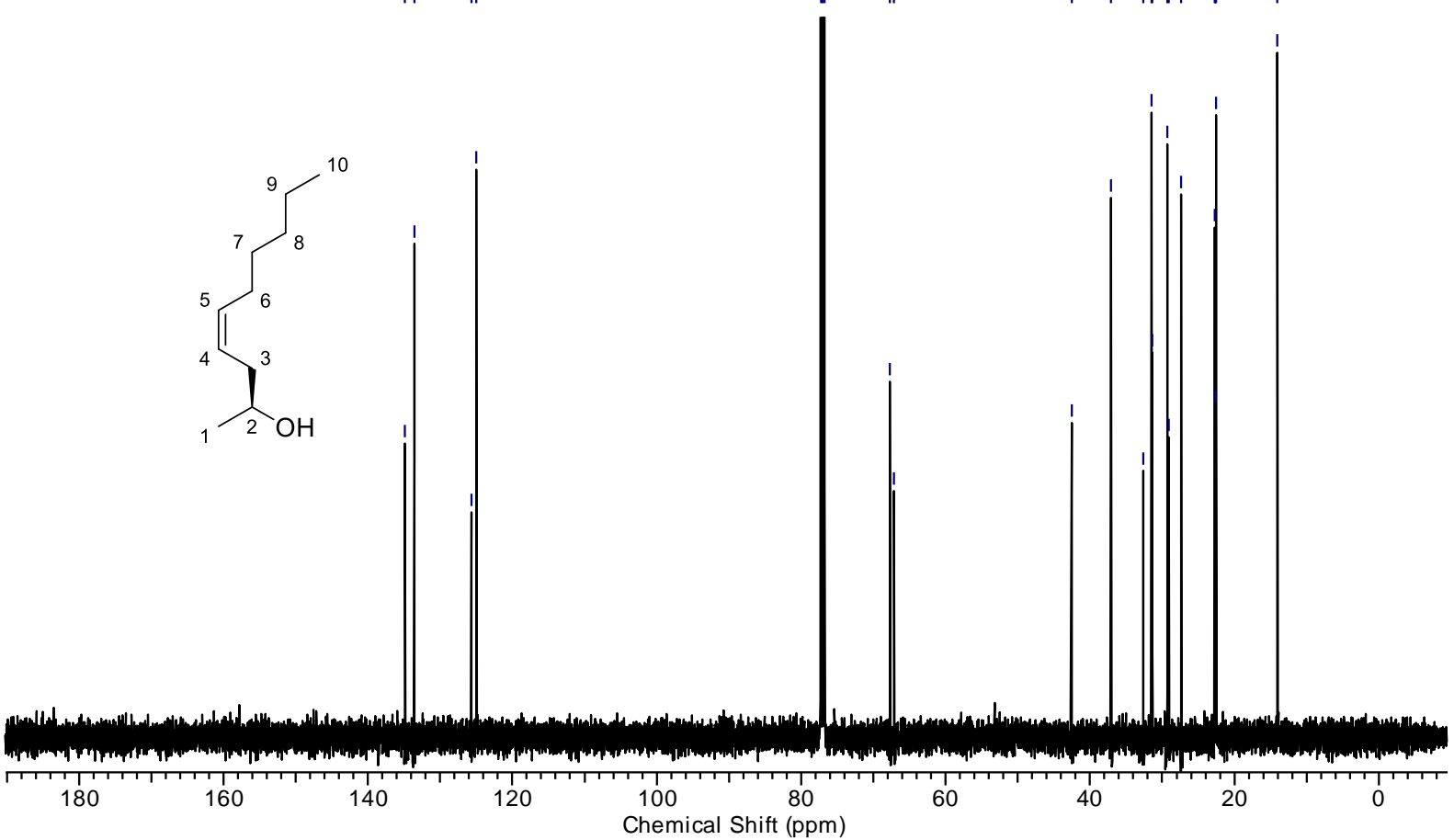


(R,Z)-1-Cyclohexylpent-3-en-1-ol (18c, mixture of $(E)$ and $(Z)$ isomers)

${ }^{1} \mathbf{H}$ NMR (500 MHz, $\left.\mathrm{CDCl}_{3}\right)$ :

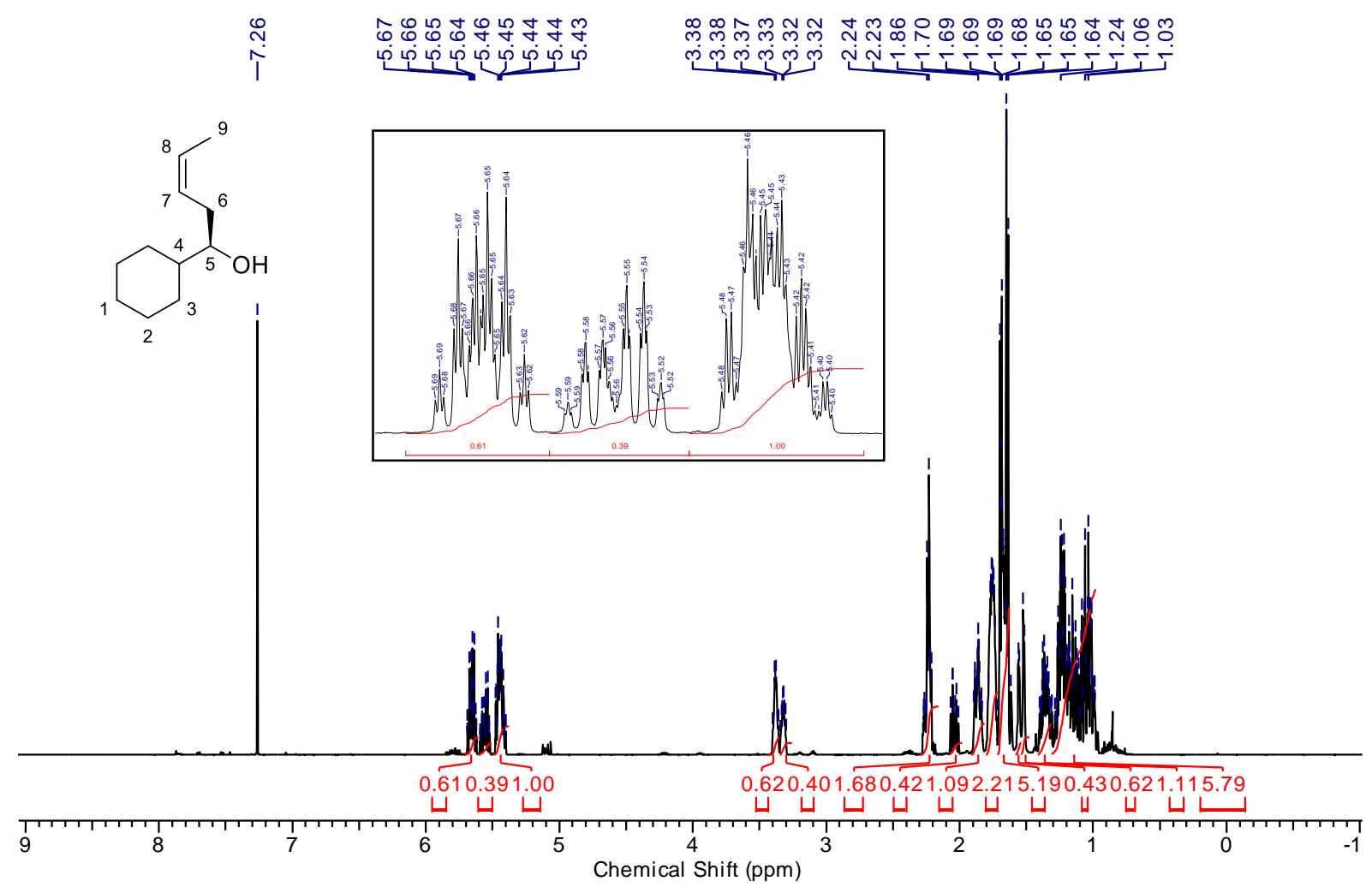

${ }^{13} \mathrm{C} \mathrm{NMR}\left(125 \mathrm{MHz}, \mathrm{CDCl}_{3}\right)$ :

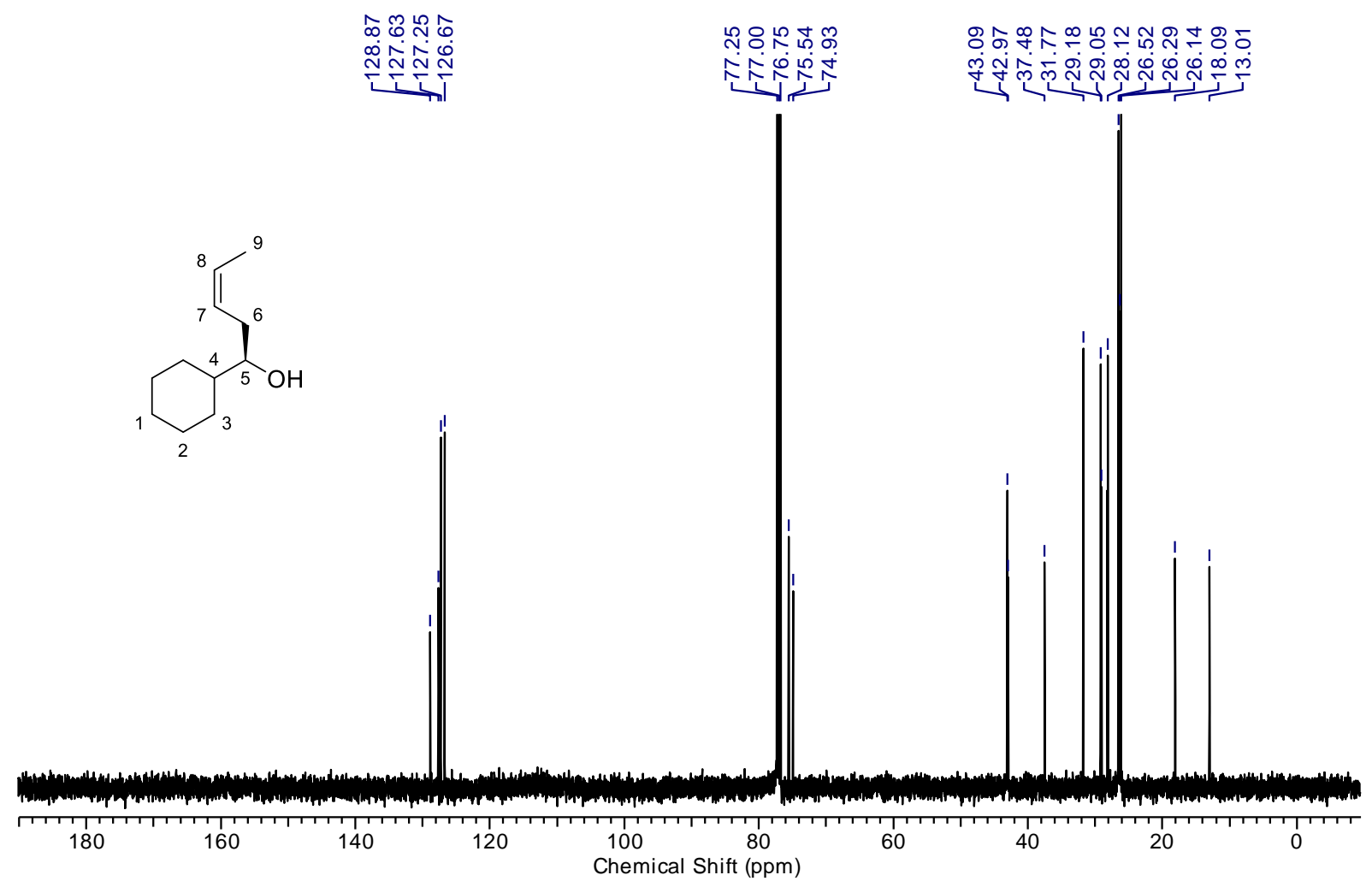


$(\boldsymbol{R}, \boldsymbol{E})$-1-Cyclohexylnon-3-en-1-ol (18d, mixture of $(E)$ and $(Z)$ isomers)

${ }^{1} \mathbf{H}$ NMR (500 MHz, $\left.\mathrm{CDCl}_{3}\right)$ :

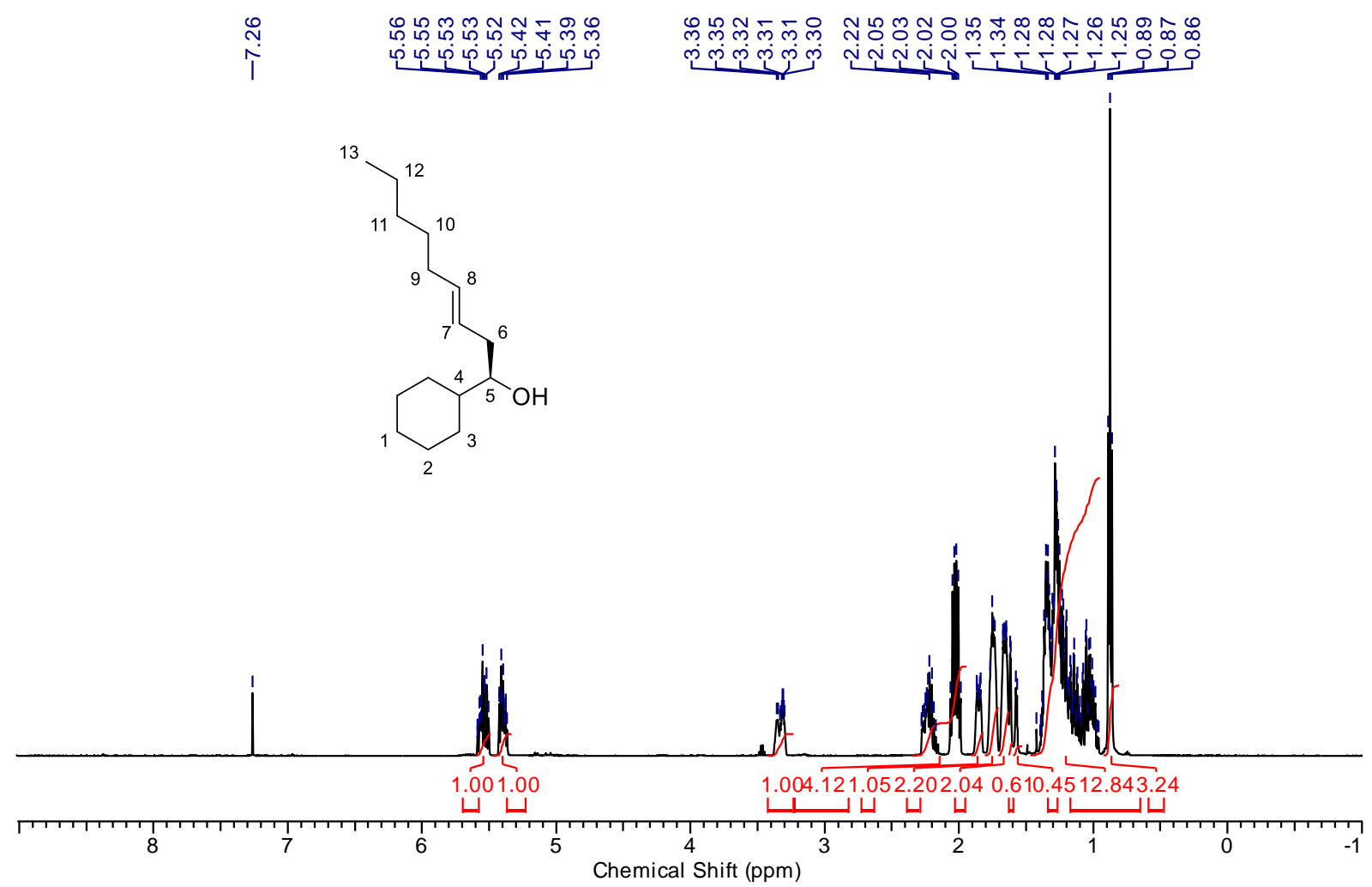

${ }^{13} \mathrm{C}$ NMR $\left(125 \mathrm{MHz}, \mathrm{CDCl}_{3}\right)$ :

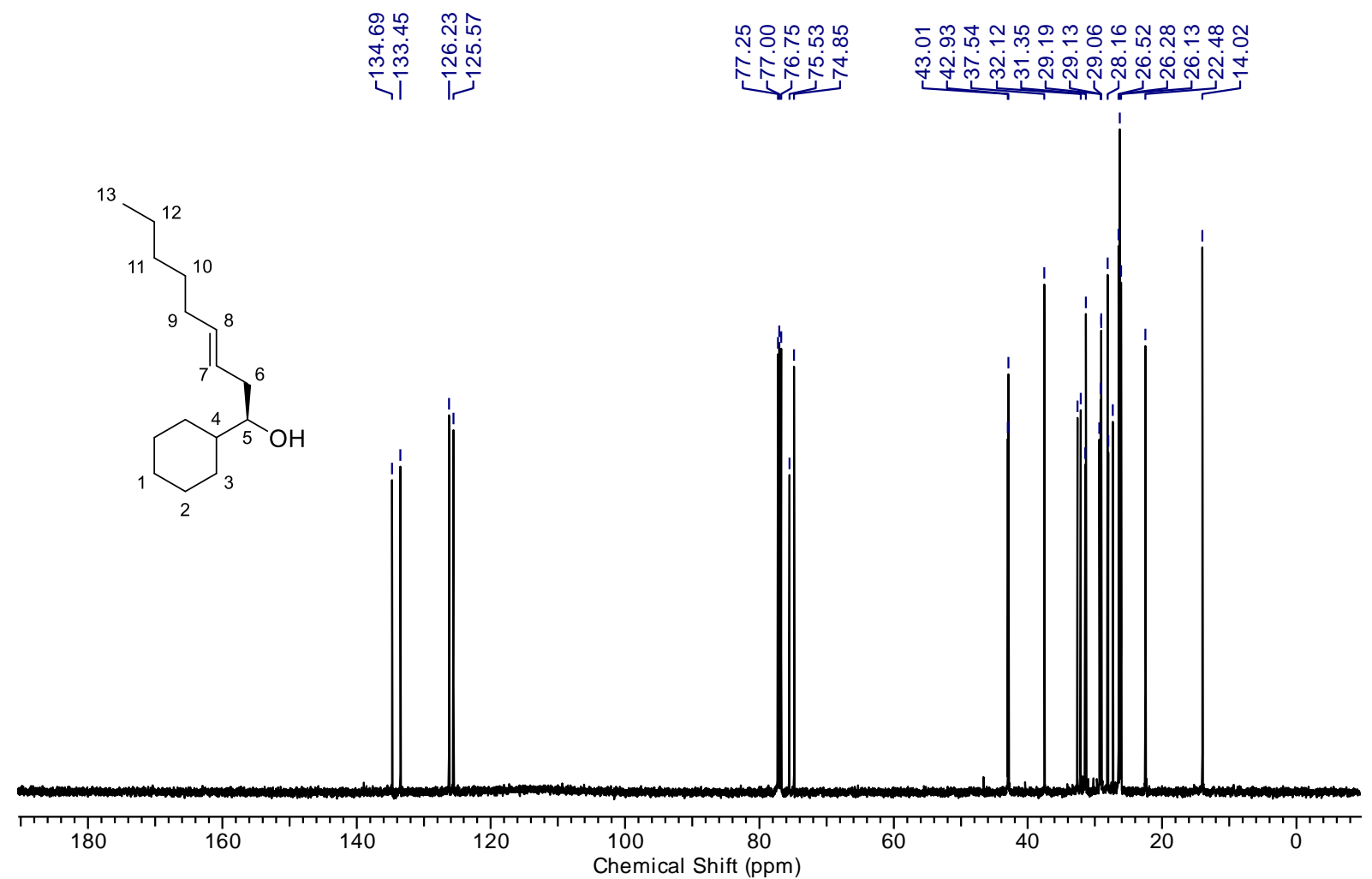




\section{GC-FID (18d)}

Column: Varian CP-Chirasil-Dex CB (25 m x $0.25 \mathrm{~mm}, 0.25 \mu \mathrm{m}$ ID)

Carrier gas: $\mathrm{N}_{2}$

$\mathrm{T}_{0}[5 \mathrm{~min}]=40^{\circ} \mathrm{C}, 5^{\circ} \mathrm{C} / \mathrm{min}$ to $200^{\circ} \mathrm{C}[5 \mathrm{~min}]$, injector $250{ }^{\circ} \mathrm{C}$, detector $275^{\circ} \mathrm{C}$

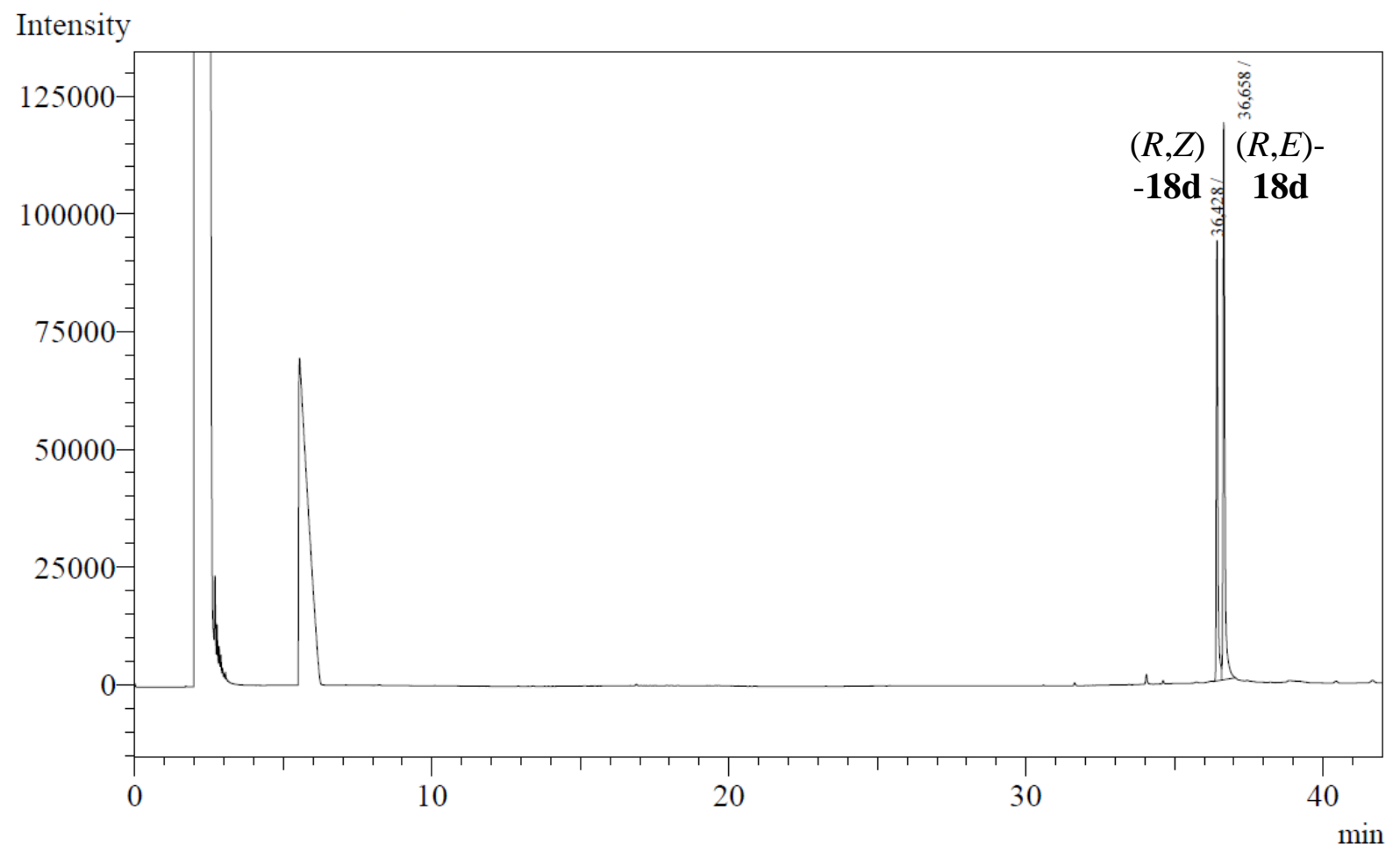

\begin{tabular}{|r|r|r|r|}
\hline Peak\# & Ret.Time & \multicolumn{1}{c|}{ Area } & \multicolumn{1}{c|}{ Area\% } \\
\hline 1 & 36.428 & 336872 & 40.7221 \\
\hline 2 & 36.658 & 490375 & 59.2779 \\
\hline Total & & 827247 & 100.0000 \\
\hline
\end{tabular}


(5R,6R,Z)-6-Ethylnon-2-en-5-ol (19c, mixture of $(E)$ and $(Z)$ isomers)

${ }^{1} \mathbf{H}$ NMR $\left(500 \mathrm{MHz}, \mathrm{CDCl}_{3}\right)$ :

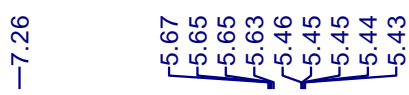

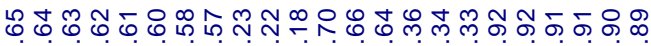

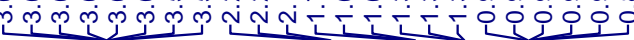
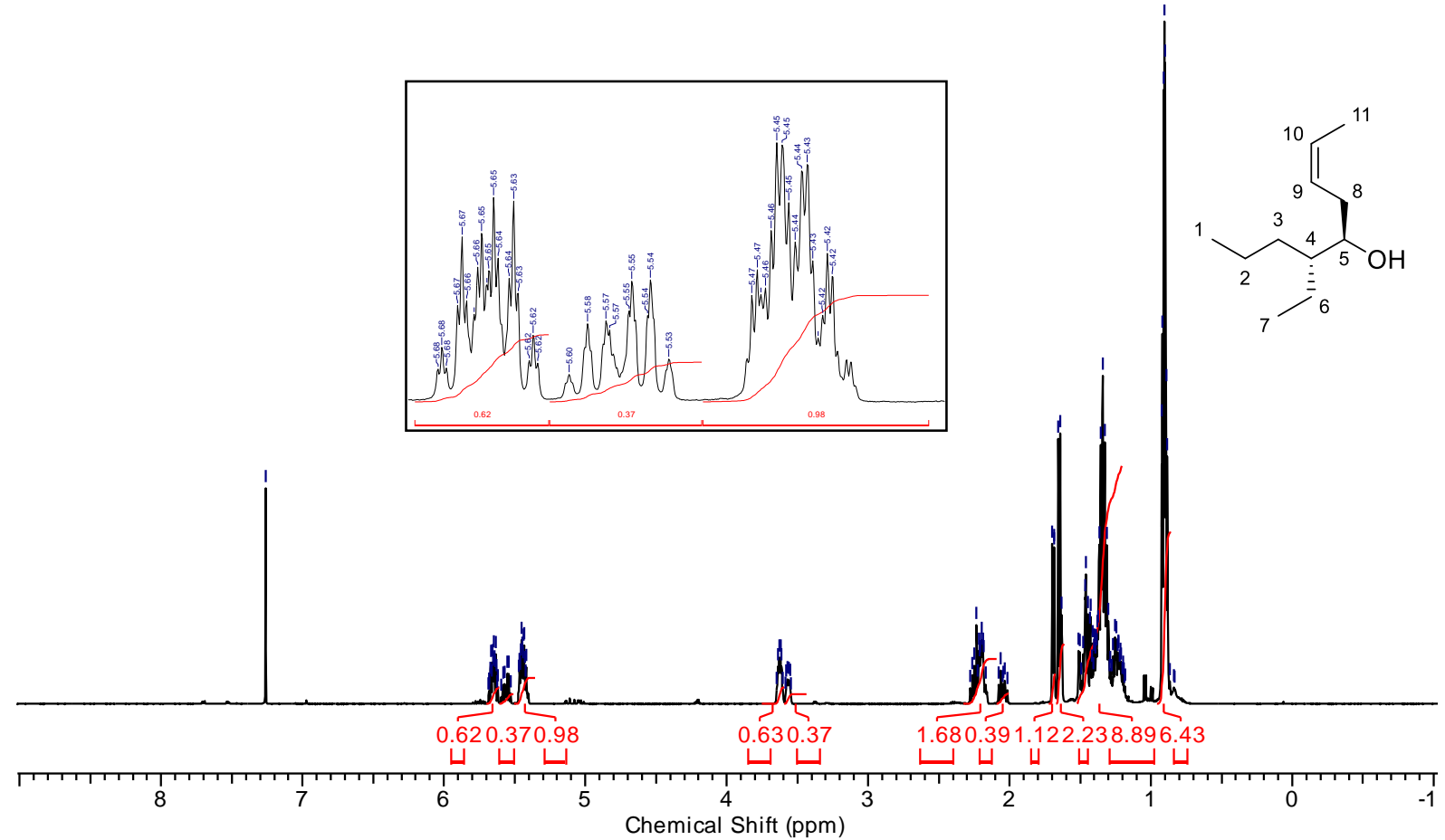

${ }^{13} \mathbf{C}$ NMR (125 MHz, $\left.\mathrm{CDCl}_{3}\right)$ :

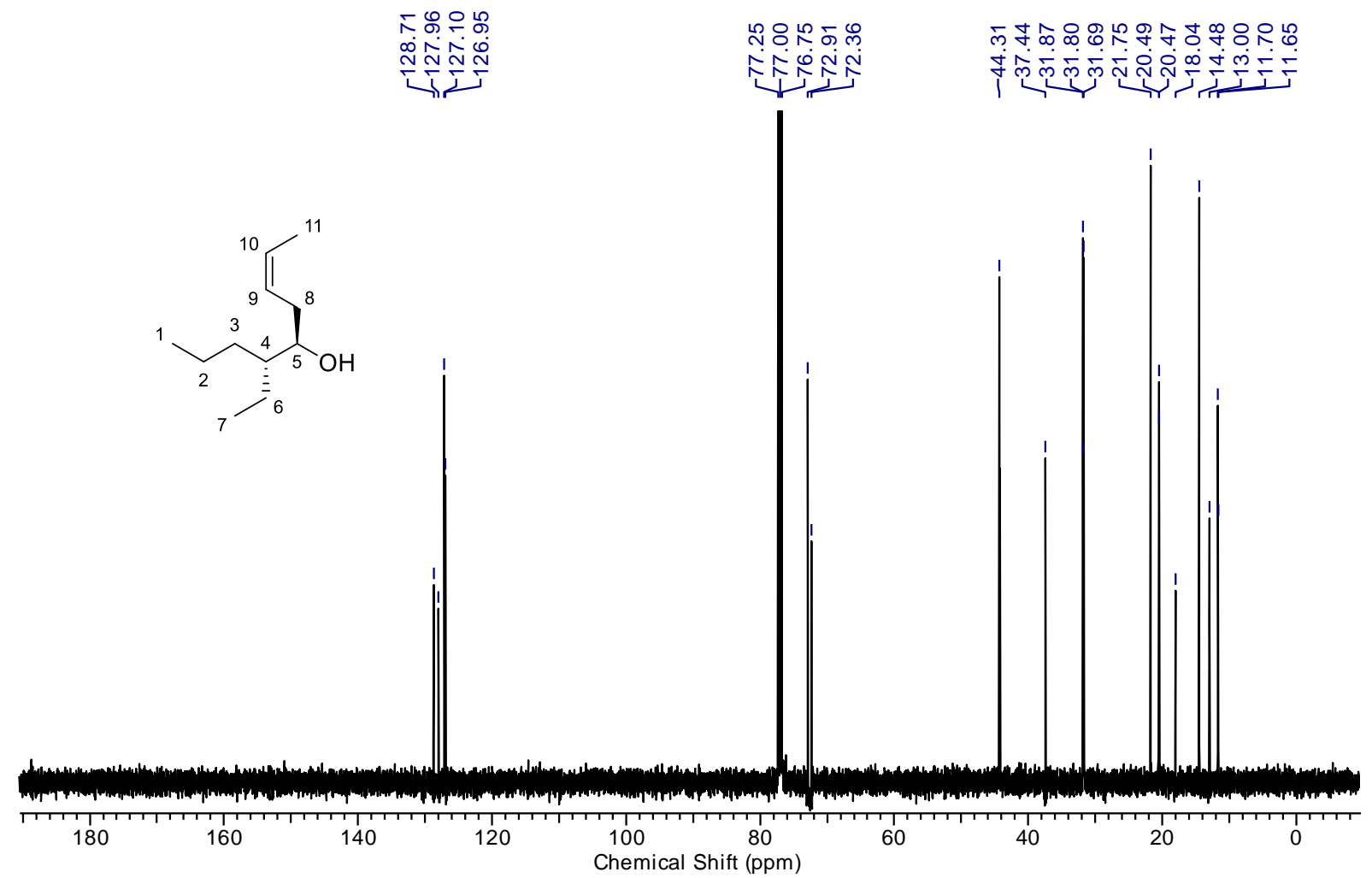


(4R,5R,E)-4-Ethyltridec-7-en-5-ol (19d, mixture of $(E)$ and $(Z)$ isomers)

${ }^{1} \mathbf{H}$ NMR $\left(500 \mathrm{MHz}, \mathrm{CDCl}_{3}\right)$ :

ị

ฮิ
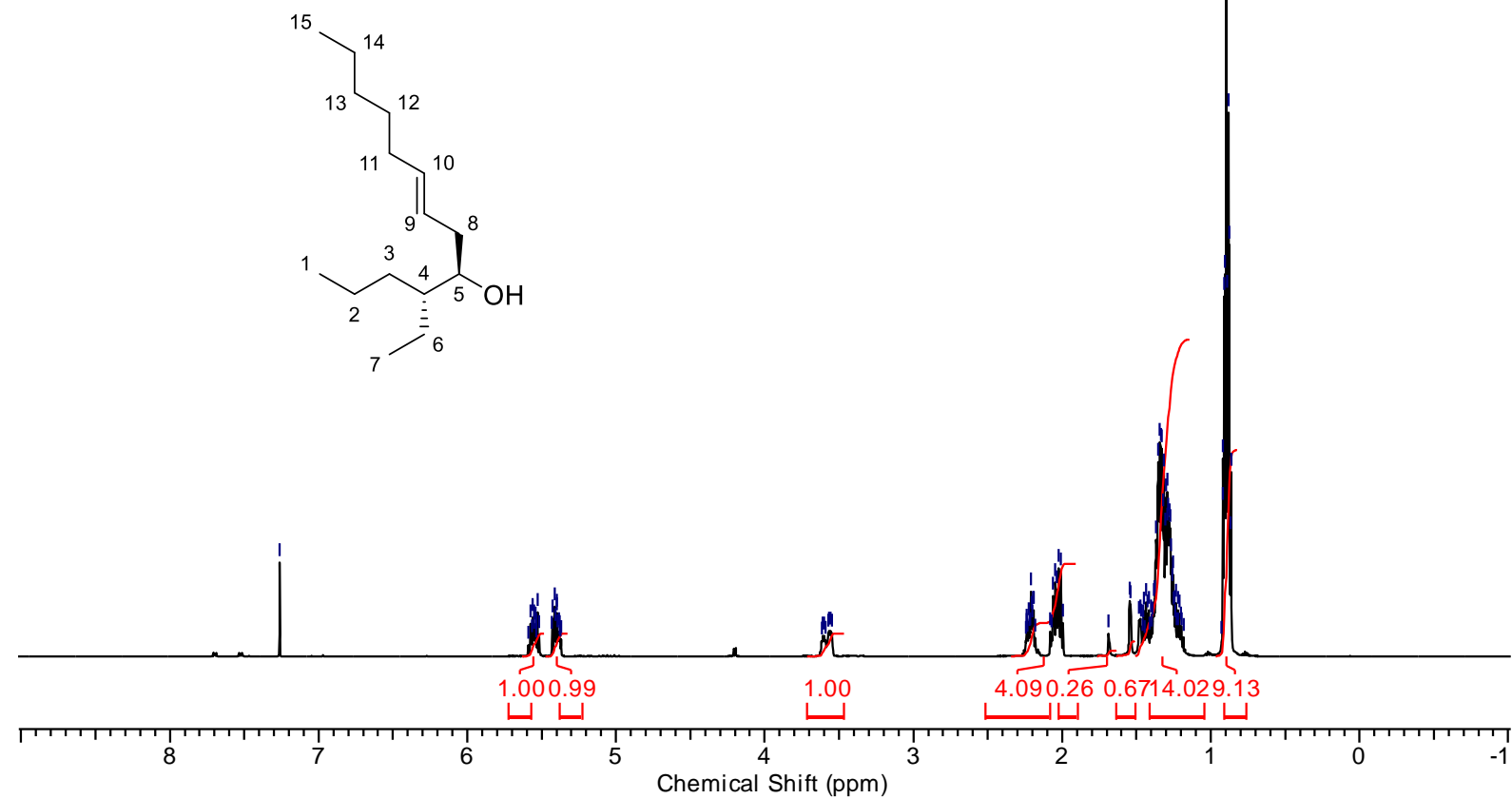

${ }^{13} \mathrm{C}$ NMR (125 MHz, $\left.\mathrm{CDCl}_{3}\right)$ :

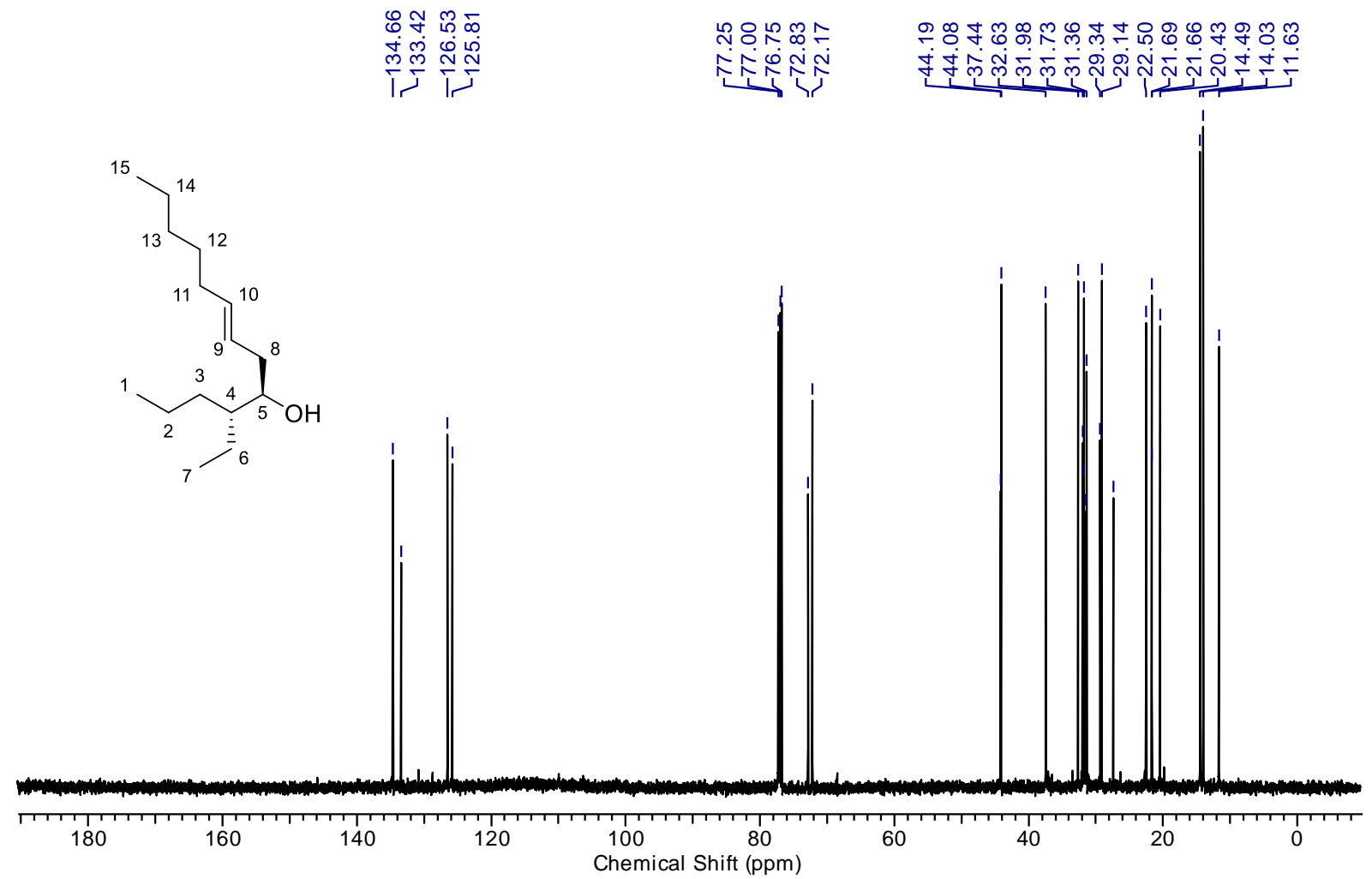




\section{GC-FID (19d)}

Column: Varian CP-Chirasil-Dex CB (25 m x $0.25 \mathrm{~mm}, 0.25 \mu \mathrm{m}$ ID)

Carrier gas: $\mathrm{N}_{2}$

$\mathrm{T}_{0}[5 \mathrm{~min}]=40^{\circ} \mathrm{C}, 5^{\circ} \mathrm{C} / \mathrm{min}$ to $200^{\circ} \mathrm{C}[5 \mathrm{~min}]$, injector $250{ }^{\circ} \mathrm{C}$, detector $275^{\circ} \mathrm{C}$

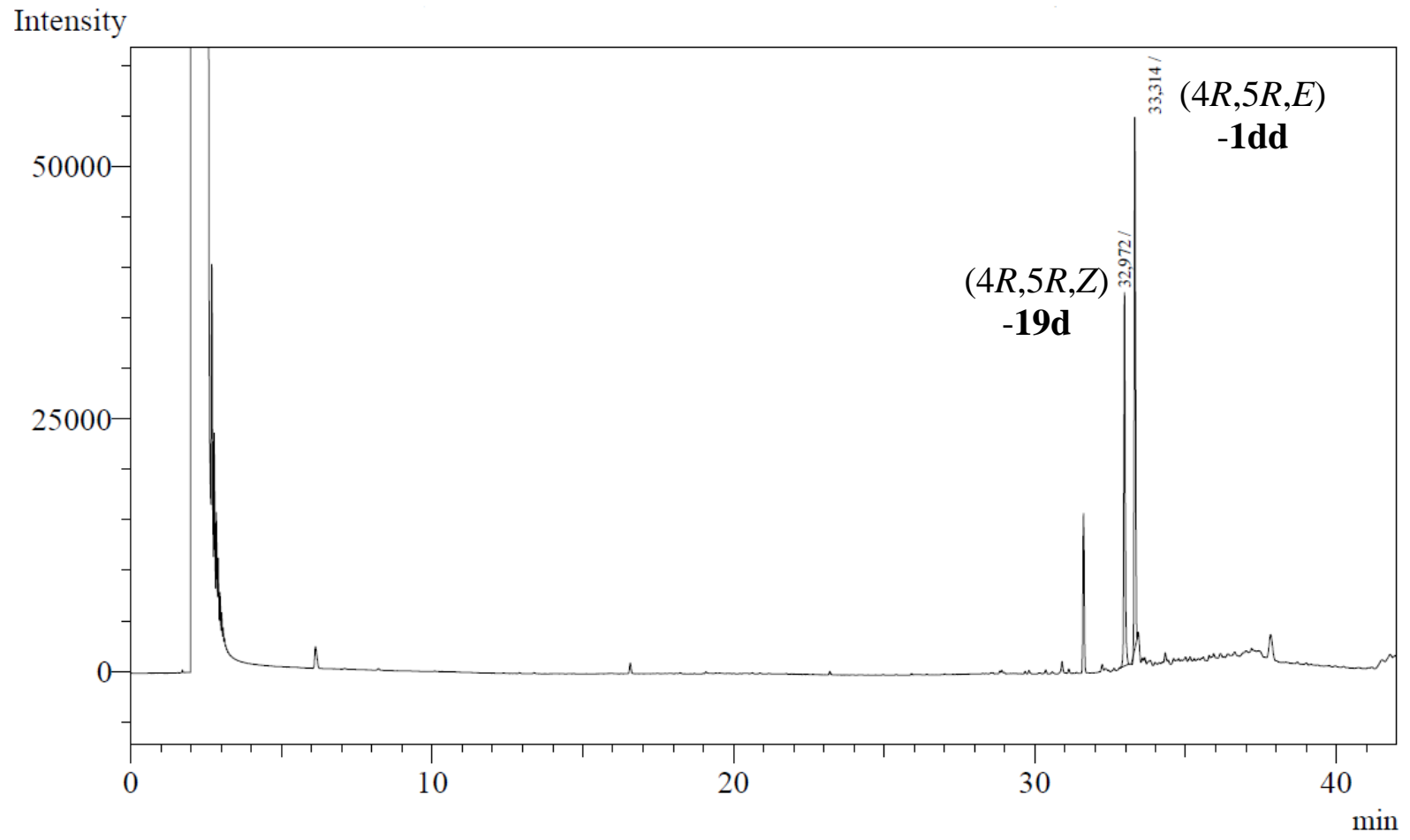

\begin{tabular}{|c|r|r|r|}
\hline Peak\# & Ret.Time & \multicolumn{1}{c|}{ Area } & \multicolumn{1}{c|}{ Area\% } \\
\hline 1 & 32.972 & 121477 & 42.7853 \\
\hline 2 & 33.314 & 162445 & 57.2147 \\
\hline Total & & 283922 & 100.0000 \\
\hline
\end{tabular}


tert-Butyl $\{[(R)-5$-Chloro-5-([4S,5S]-4,5-dicyclohexyl-1,3,2-dioxaborolan-2-yl)pentyl]oxy $\}$ dimethylsilane (20-Cl, crude product)

${ }^{1} \mathbf{H}$ NMR (400 MHz, $\left.\mathrm{CDCl}_{3}\right)$ :

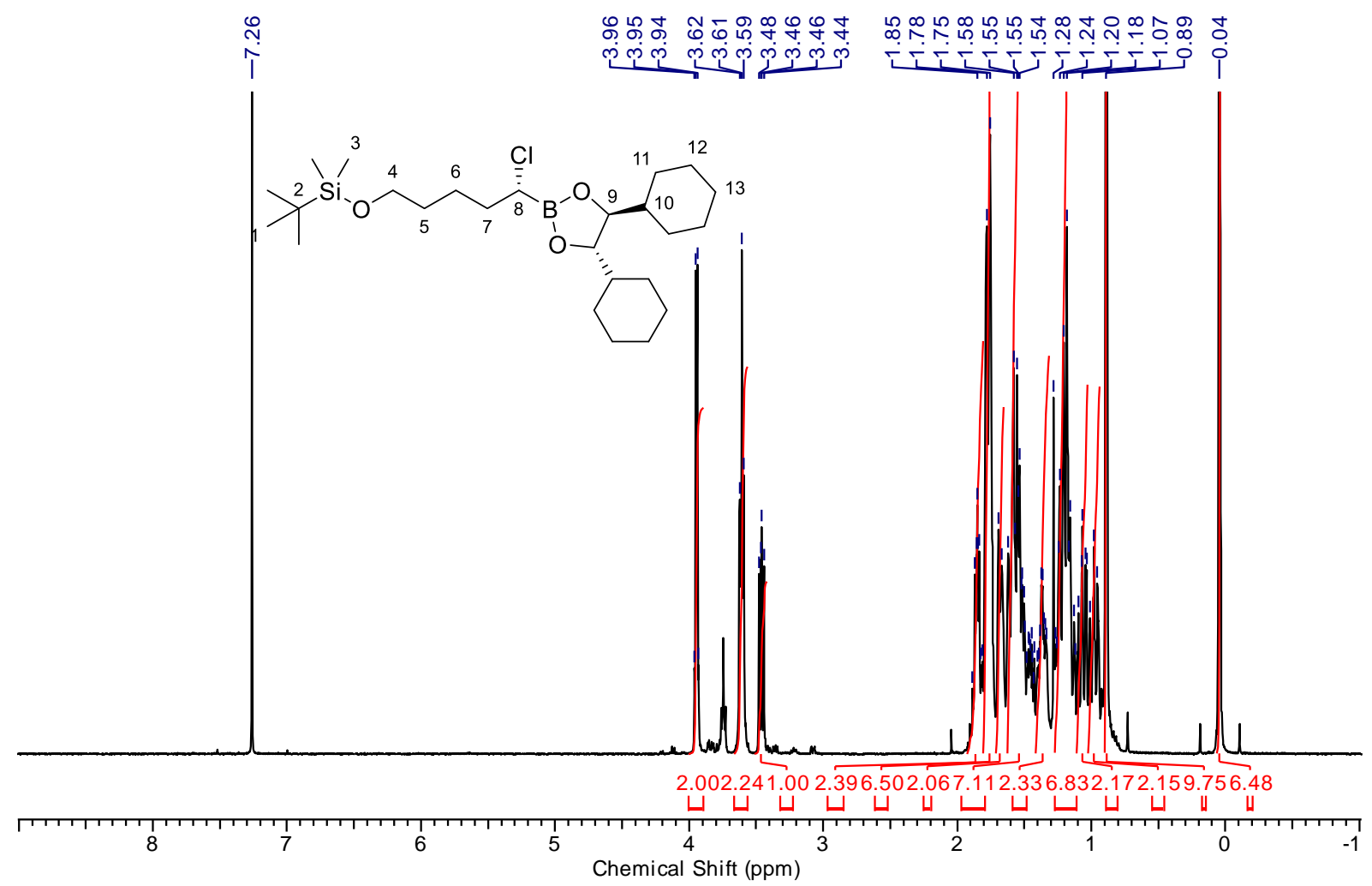

${ }^{13}$ C NMR (100 MHz, $\left.\mathrm{CDCl}_{3}\right)$ :

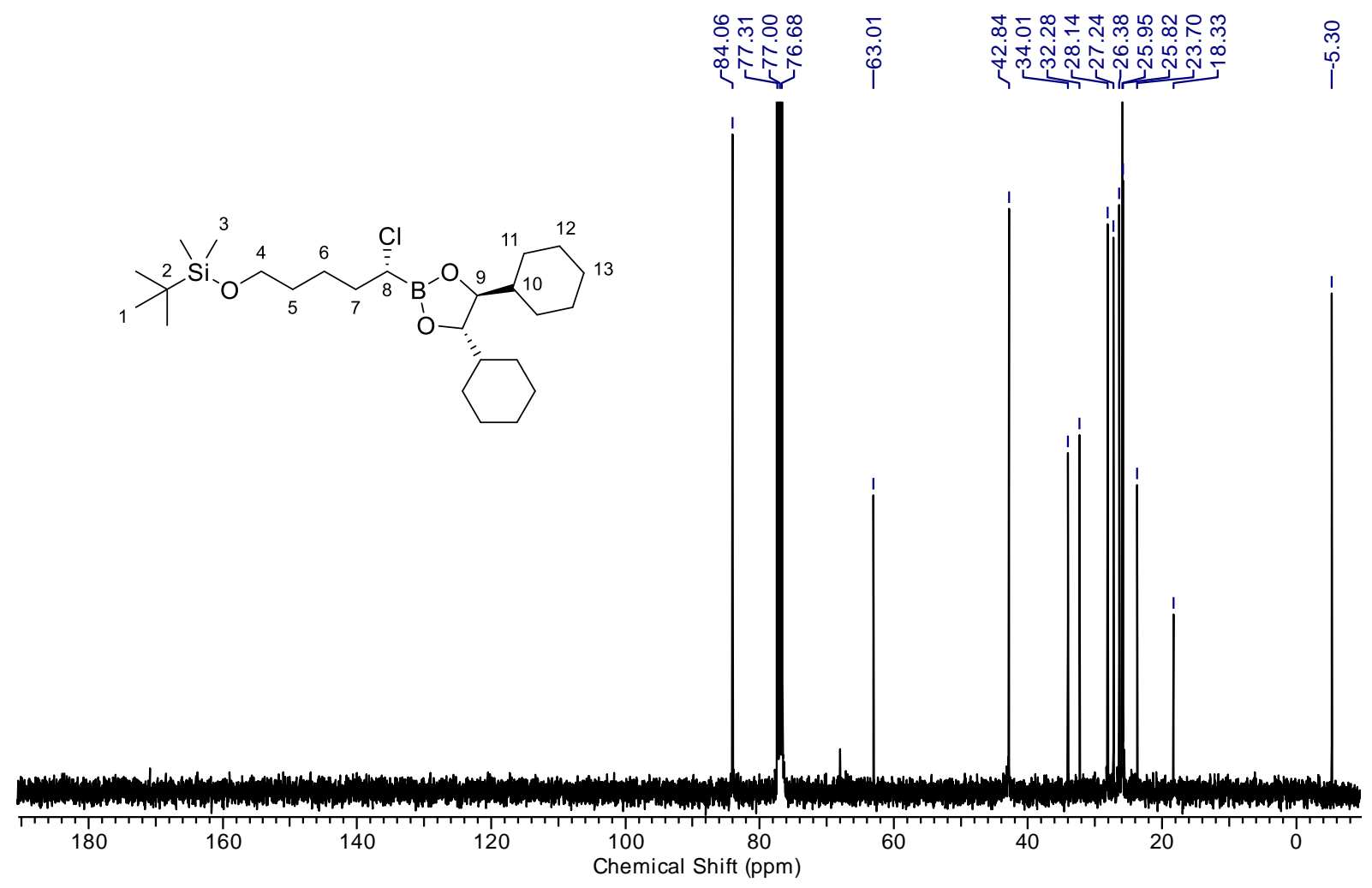


(4S,5S)-4,5-Dicyclohexyl-2-[(S,E)-7,7-dimethyl-1-phenyloct-5-en-3-yl]-1,3,2dioxaborolane (21)

${ }^{1} \mathbf{H}$ NMR (500 MHz, $\left.\mathrm{CDCl}_{3}\right)$ :

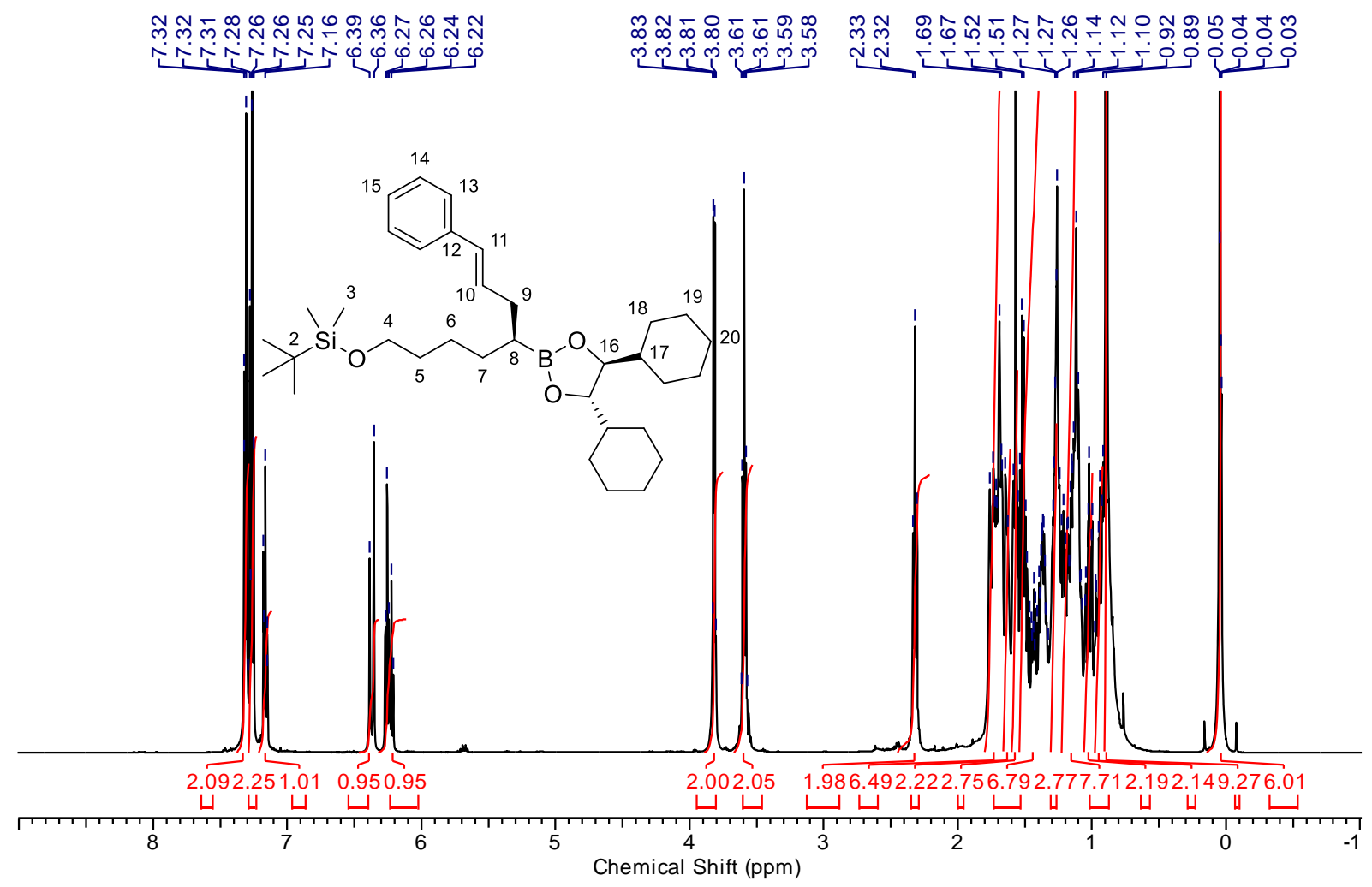

${ }^{13} \mathrm{C}$ NMR $\left(125 \mathrm{MHz}, \mathrm{CDCl}_{3}\right)$ :

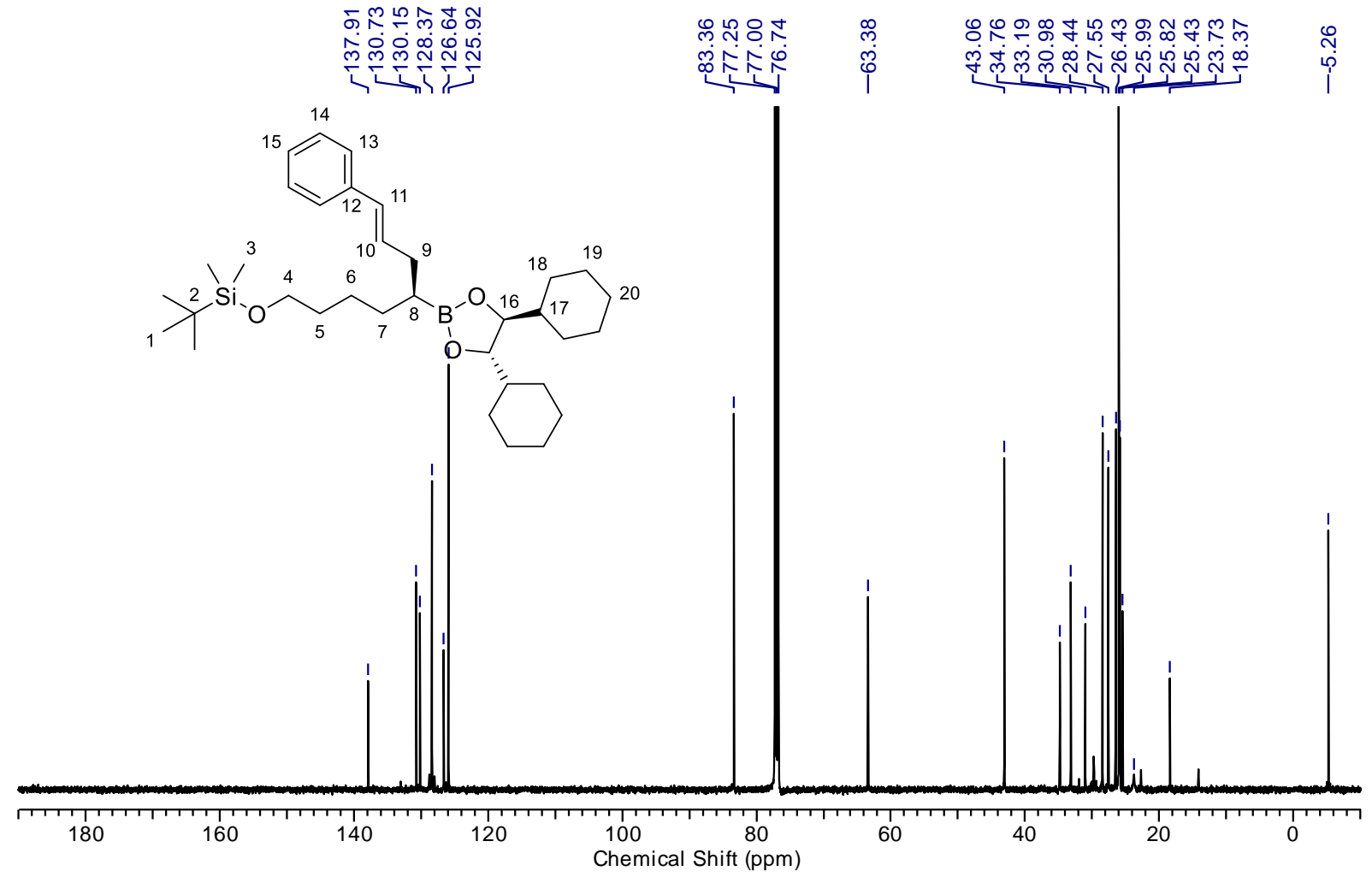




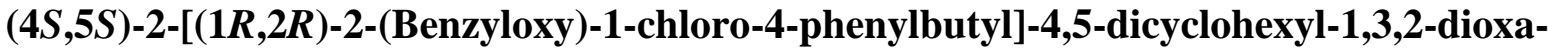
borolane (22)

${ }^{1} \mathbf{H}$ NMR $\left(400 \mathrm{MHz}, \mathrm{CDCl}_{3}\right)$ :

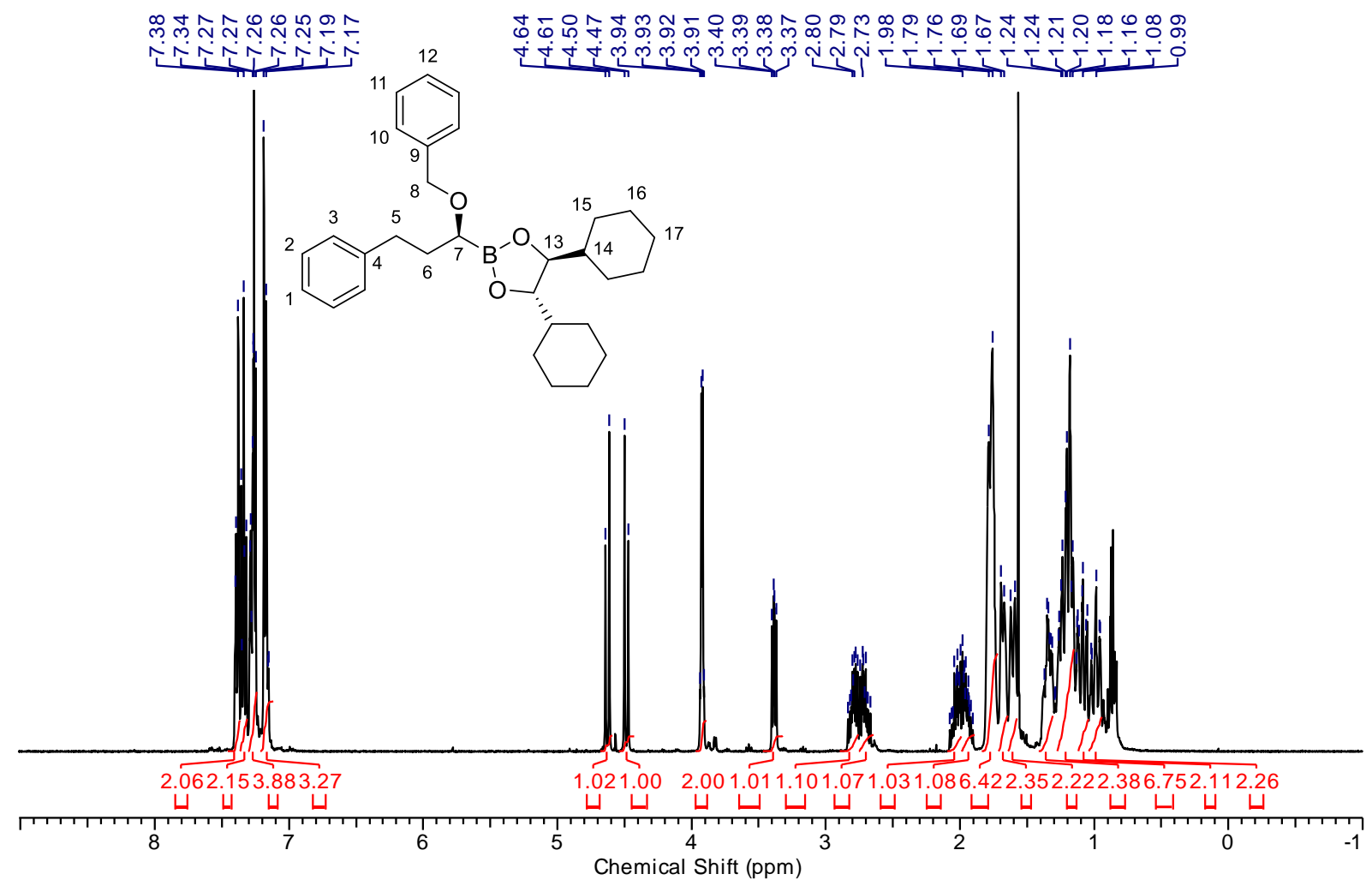

${ }^{13} \mathrm{C}$ NMR (100 MHz, $\left.\mathrm{CDCl}_{3}\right)$ :

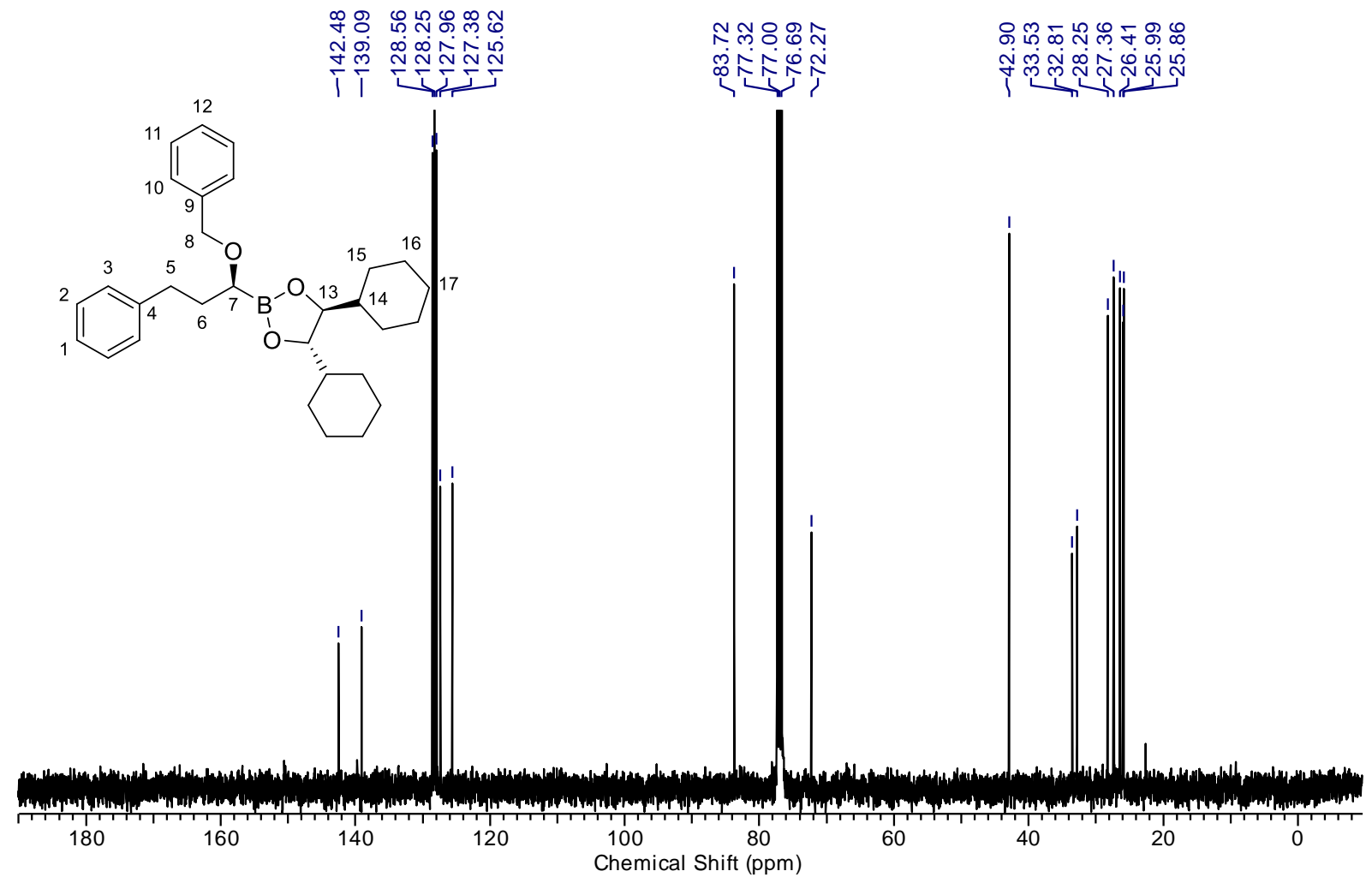


(4S,5S)-2-[(1R,2R)-2-(Benzyloxy)-1-chloro-4-phenylbutyl]-4,5-dicyclohexyl-1,3,2dioxaborolane ( 23 , crude product)

${ }^{1} \mathbf{H}$ NMR $\left(500 \mathrm{MHz}, \mathrm{CDCl}_{3}\right)$ :

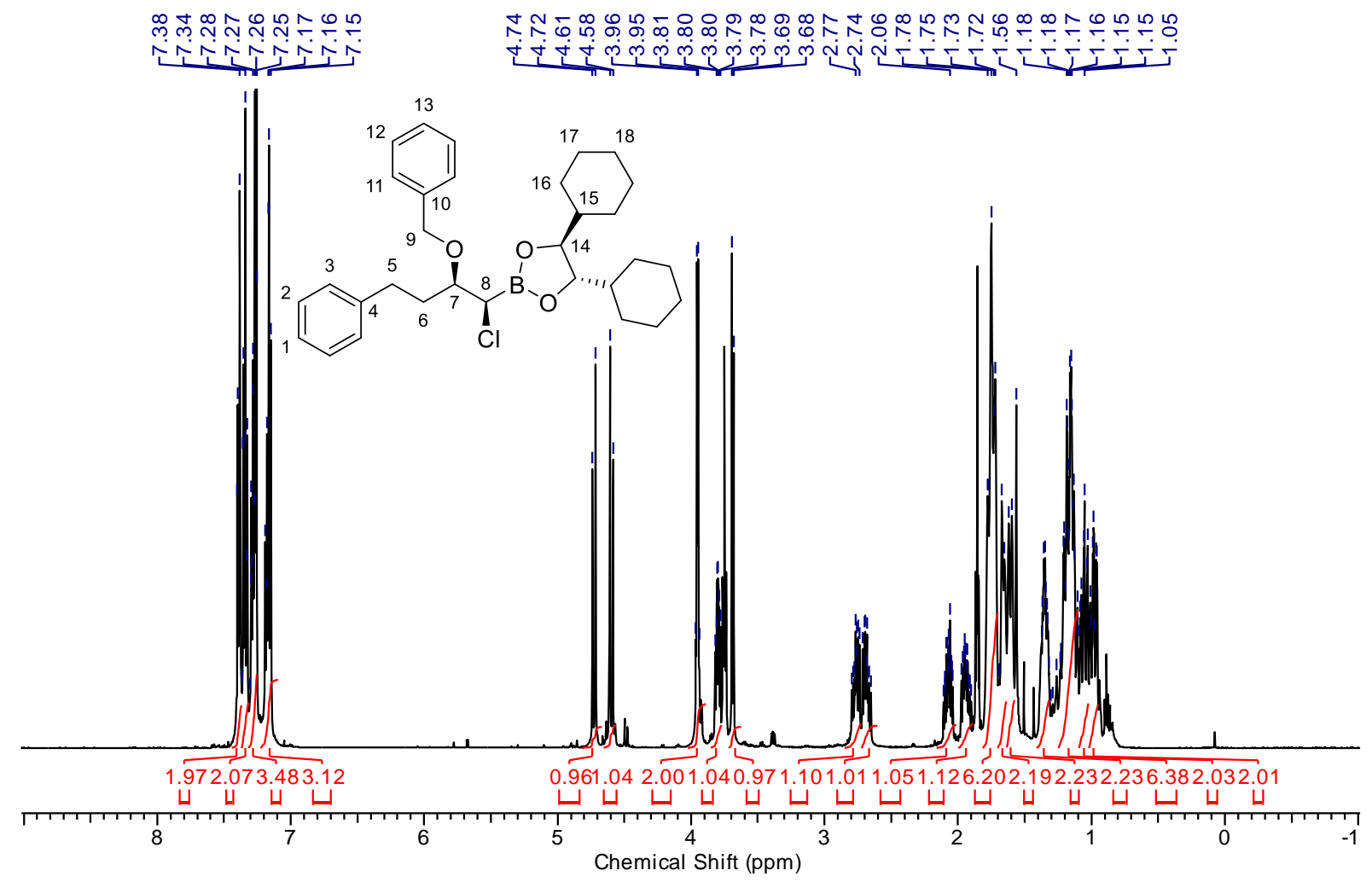

${ }^{13} \mathrm{C}$ NMR (125 MHz, $\left.\mathrm{CDCl}_{3}\right)$ :

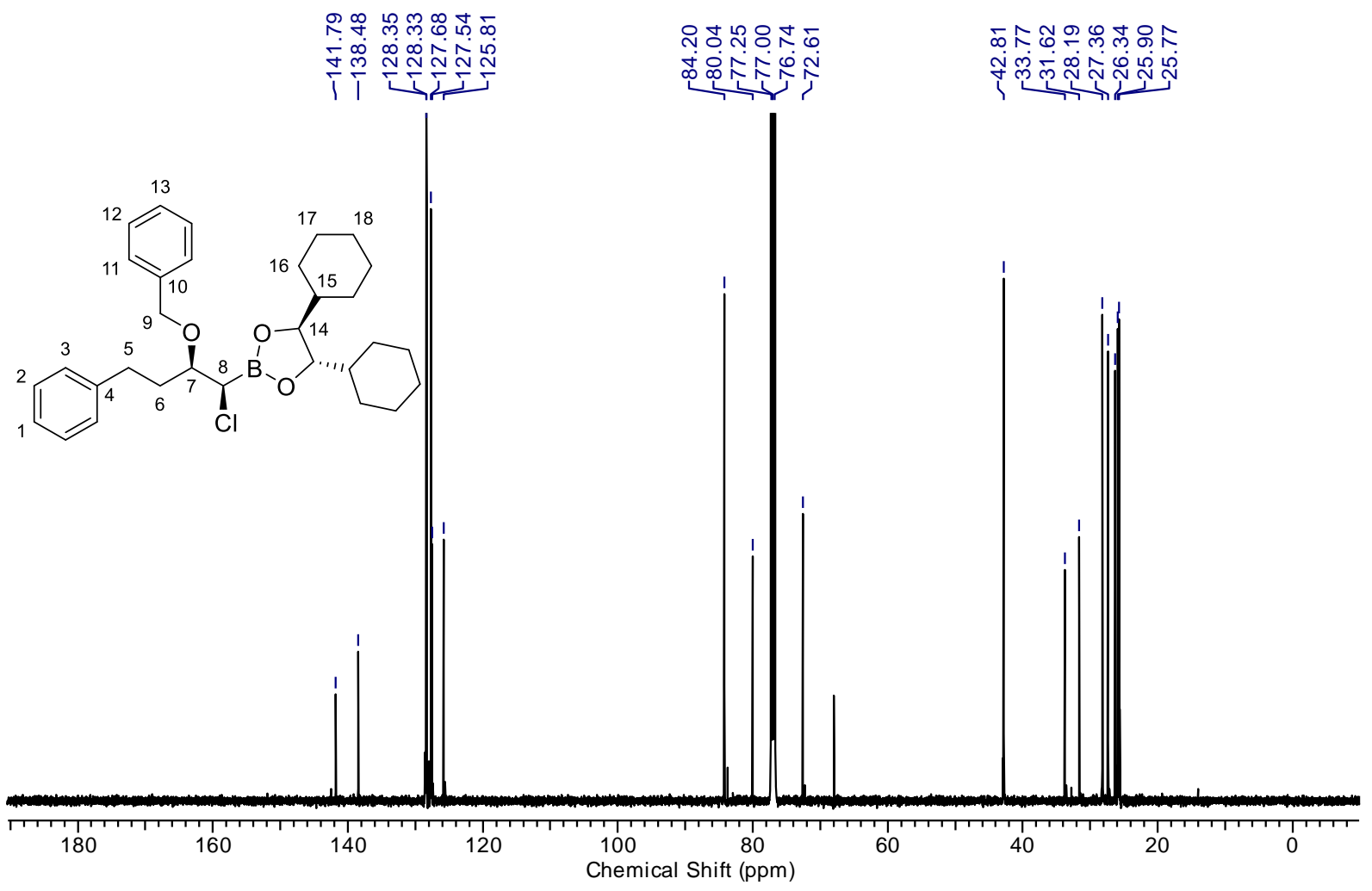


$(4 S, 5 S)-2-[(4 R, 5 R, E)-5-(B e n z y l o x y)-1,7-d i p h e n y l h e p t-1-e n-4-y l]-4,5-d i c y c l o h e x y l-1,3,2-$ dioxaborolane (24)

${ }^{1} \mathbf{H}$ NMR $\left(500 \mathrm{MHz}, \mathrm{CDCl}_{3}\right)$ :

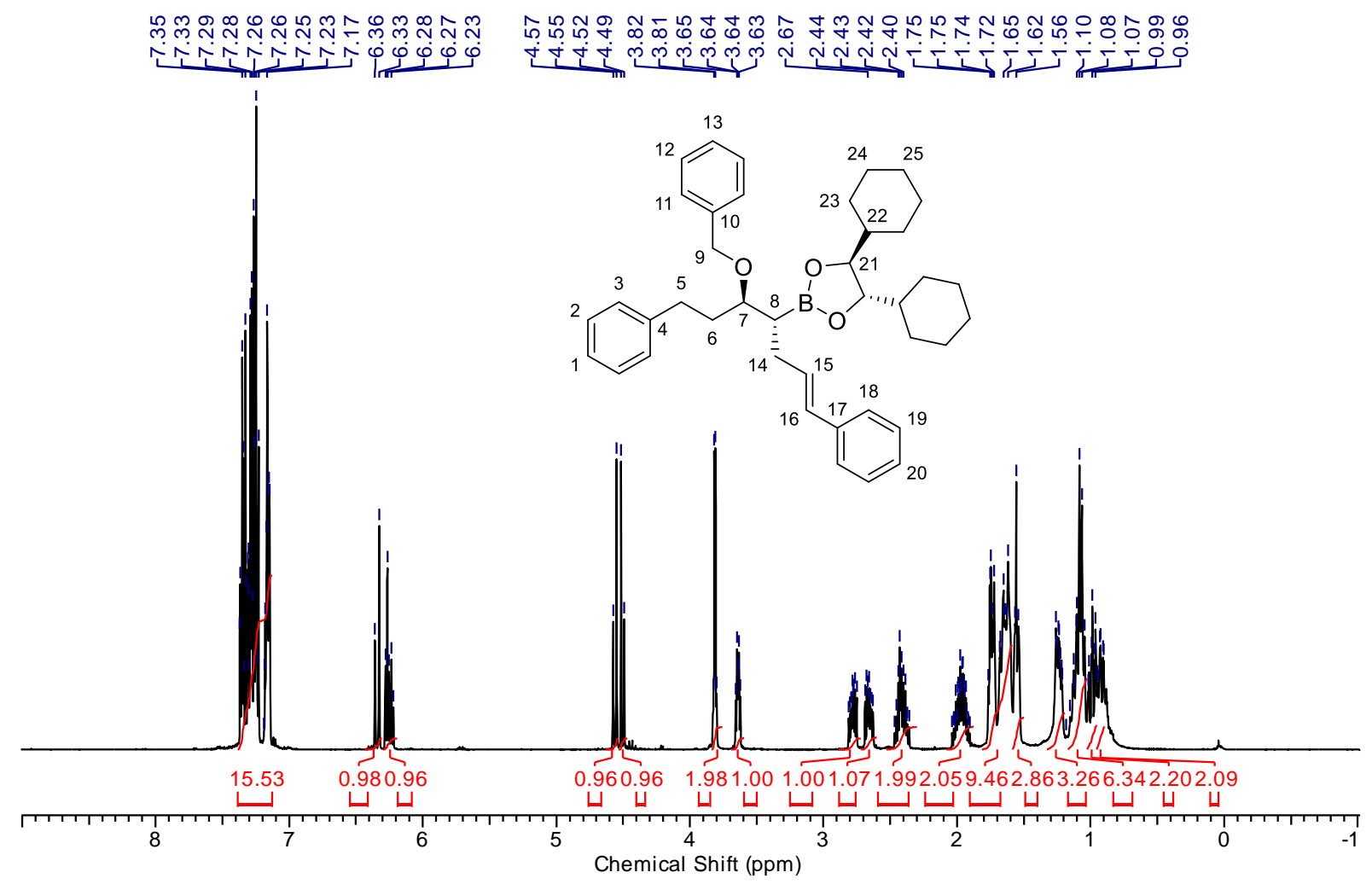

${ }^{13}$ C NMR (125 MHz, $\left.\mathrm{CDCl}_{3}\right)$ :

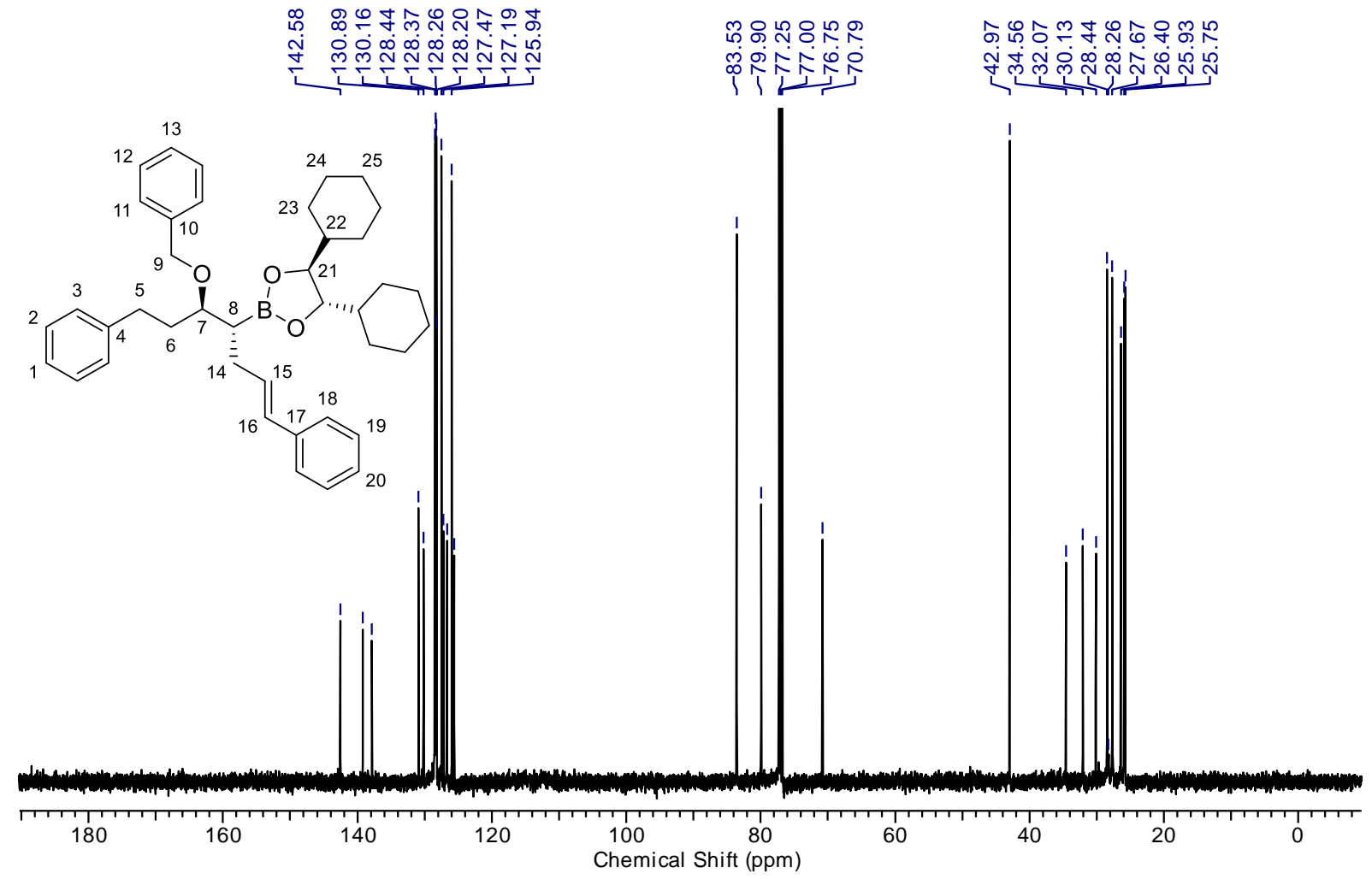


(4S,5S)-2-[(S)-2-(Benzyloxy)-4-phenylbutyl]-4,5-dicyclohexyl-1,3,2-dioxaborolane (23-H) ${ }^{1} \mathbf{H}$ NMR (500 MHz, $\left.\mathrm{CDCl}_{3}\right)$ :

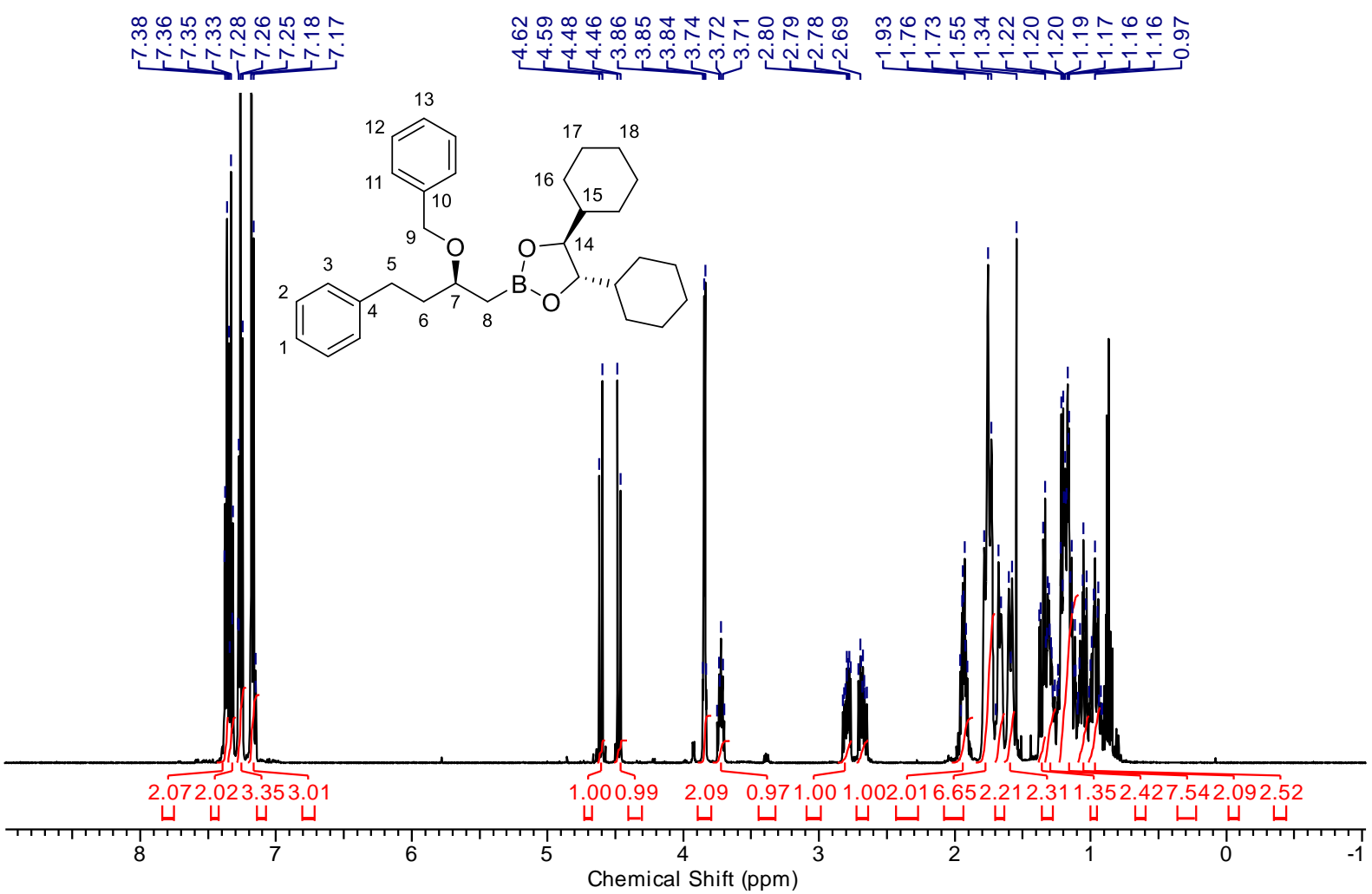

${ }^{13} \mathrm{C}$ NMR (125 MHz, $\left.\mathrm{CDCl}_{3}\right)$ :

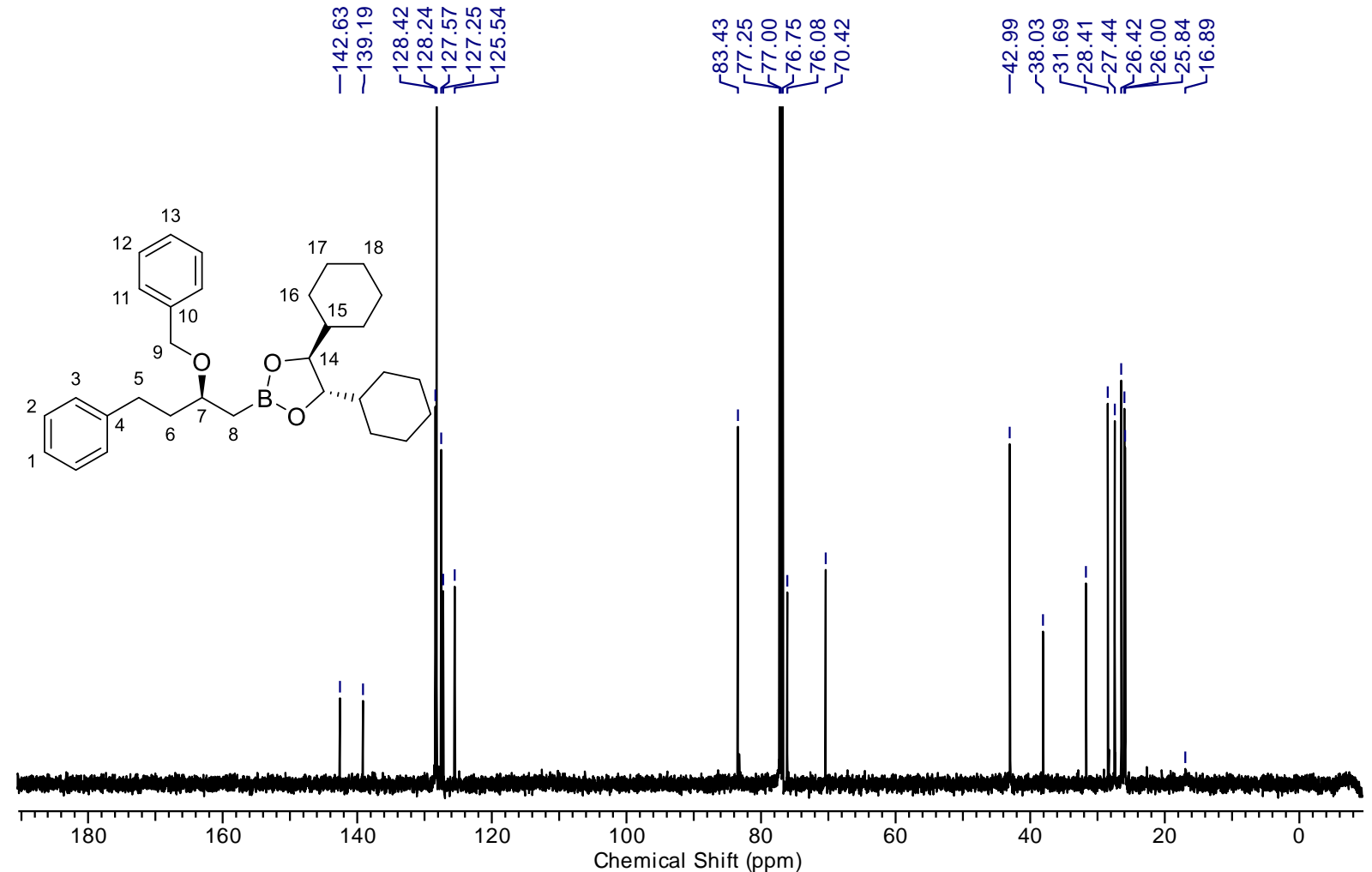


(4S,5S)-2-[(1R,3R)-3-(Benzyloxy)-1-chloro-5-phenylpentyl]-4,5-dicyclohexyl-1,3,2dioxaborolane (25, crude product)

${ }^{1} \mathbf{H}$ NMR (500 MHz, $\left.\mathrm{CDCl}_{3}\right)$ :

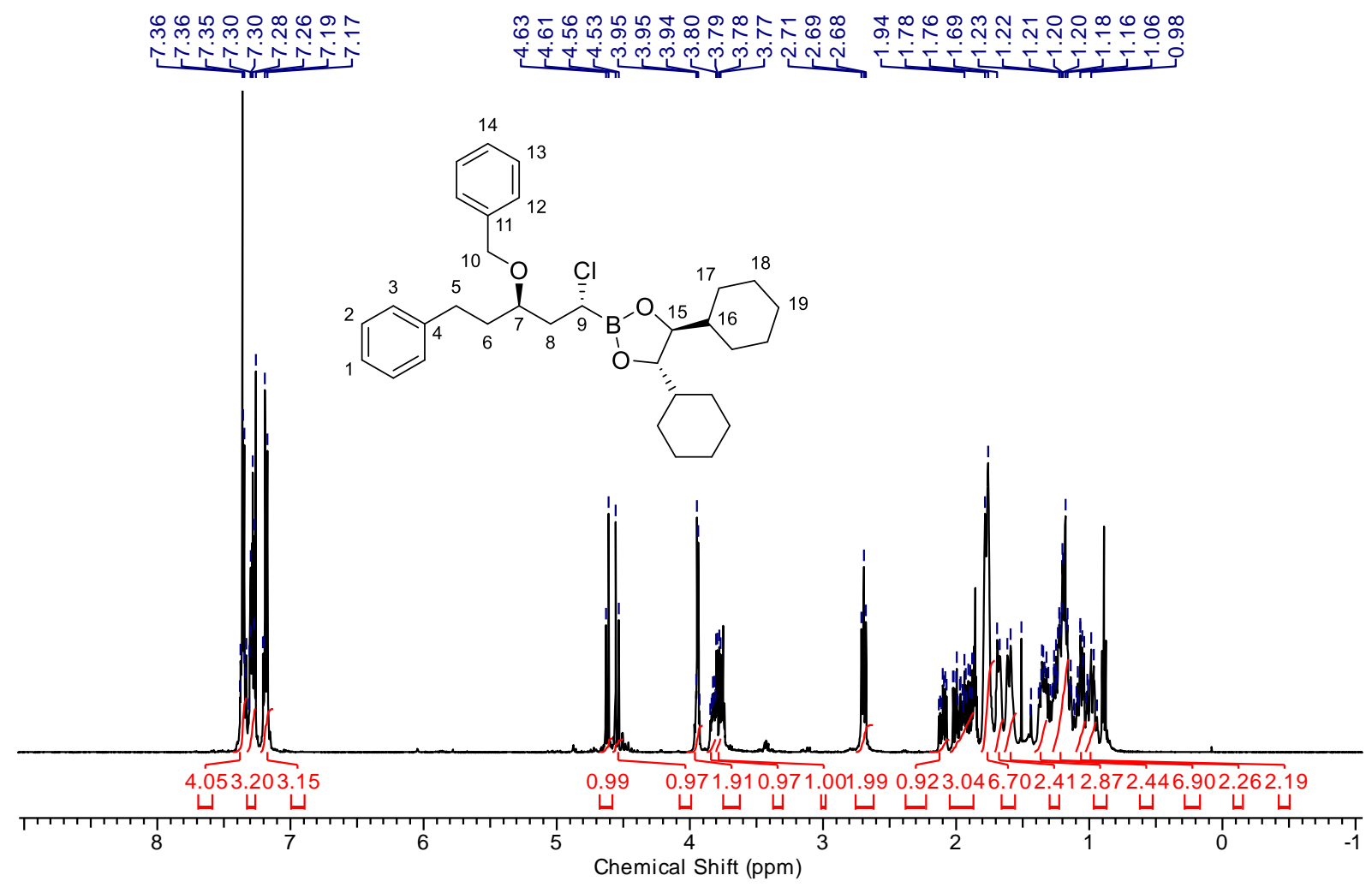

${ }^{13} \mathrm{C}$ NMR (125 MHz, $\left.\mathrm{CDCl}_{3}\right)$ :

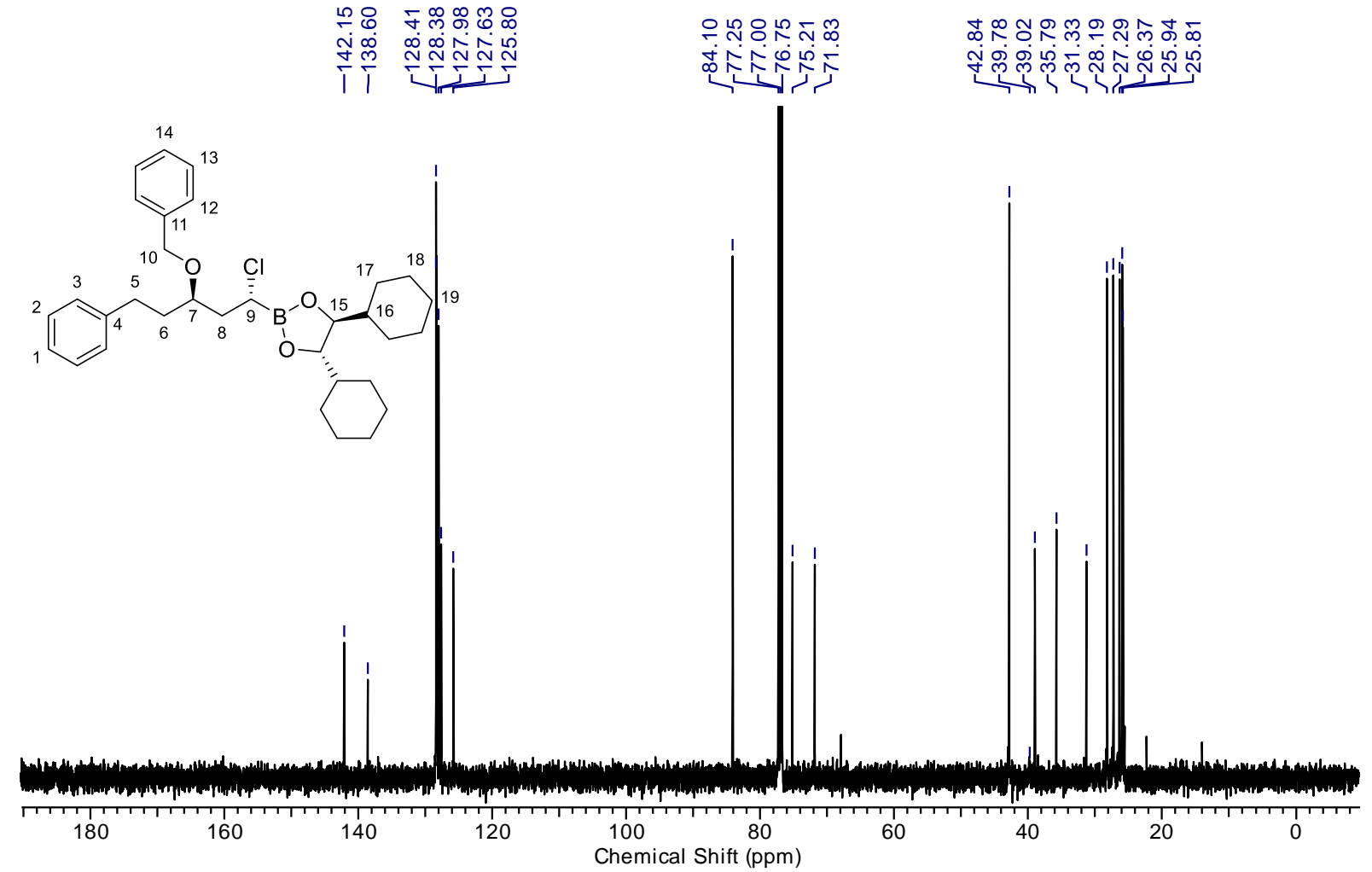


$(4 S, 5 S)-2-[(4 R, 6 R, E)-6-(B e n z y l o x y)-1,8-d i p h e n y l o c t-1-e n-4-y l]-4,5-d i c y c l o h e x y l-1,3,2-$ dioxaborolane (26)

${ }^{1} \mathbf{H}$ NMR $\left(500 \mathrm{MHz}, \mathrm{CDCl}_{3}\right)$ :

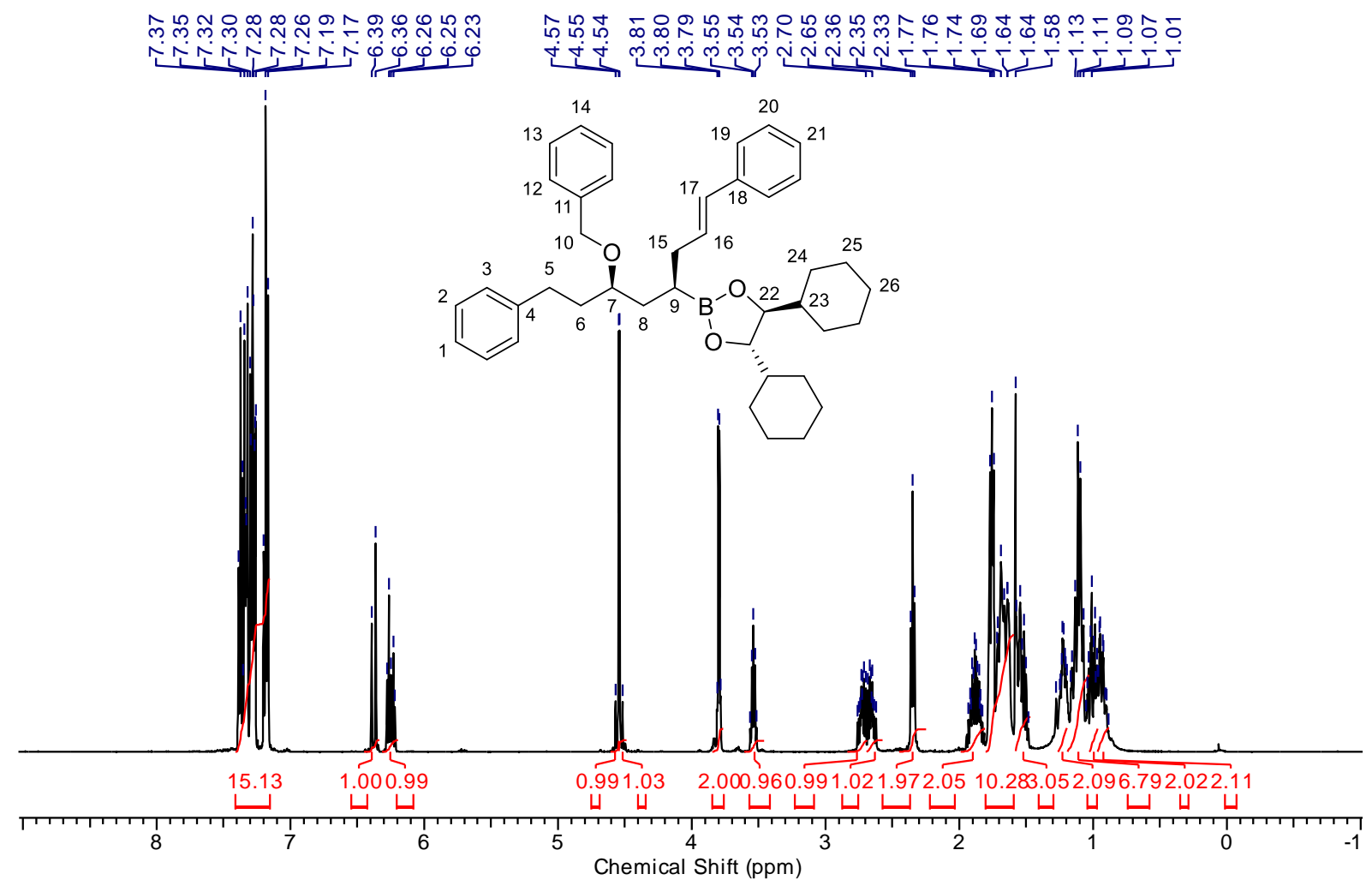

${ }^{13}$ C NMR (125 MHz, $\left.\mathrm{CDCl}_{3}\right)$ :

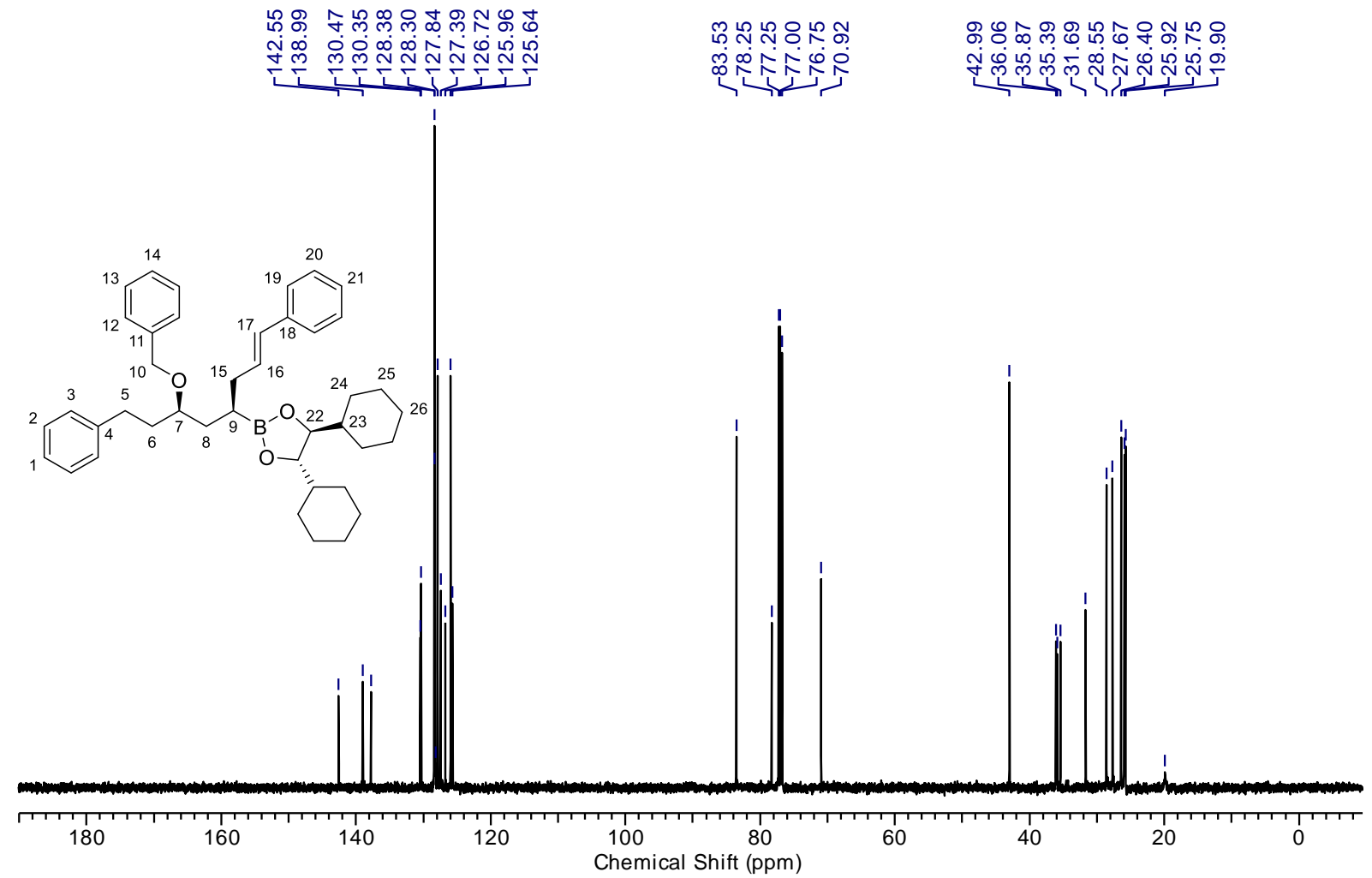


(4S,5S)-4,5-Dicyclohexyl-2-[(3R,4S,E)-4-phenethyl-7-phenylhept-6-en-3-yl]-1,3,2dioxaborolane (27)

${ }^{1} \mathbf{H}$ NMR (500 MHz, $\left.\mathrm{CDCl}_{3}\right)$ :

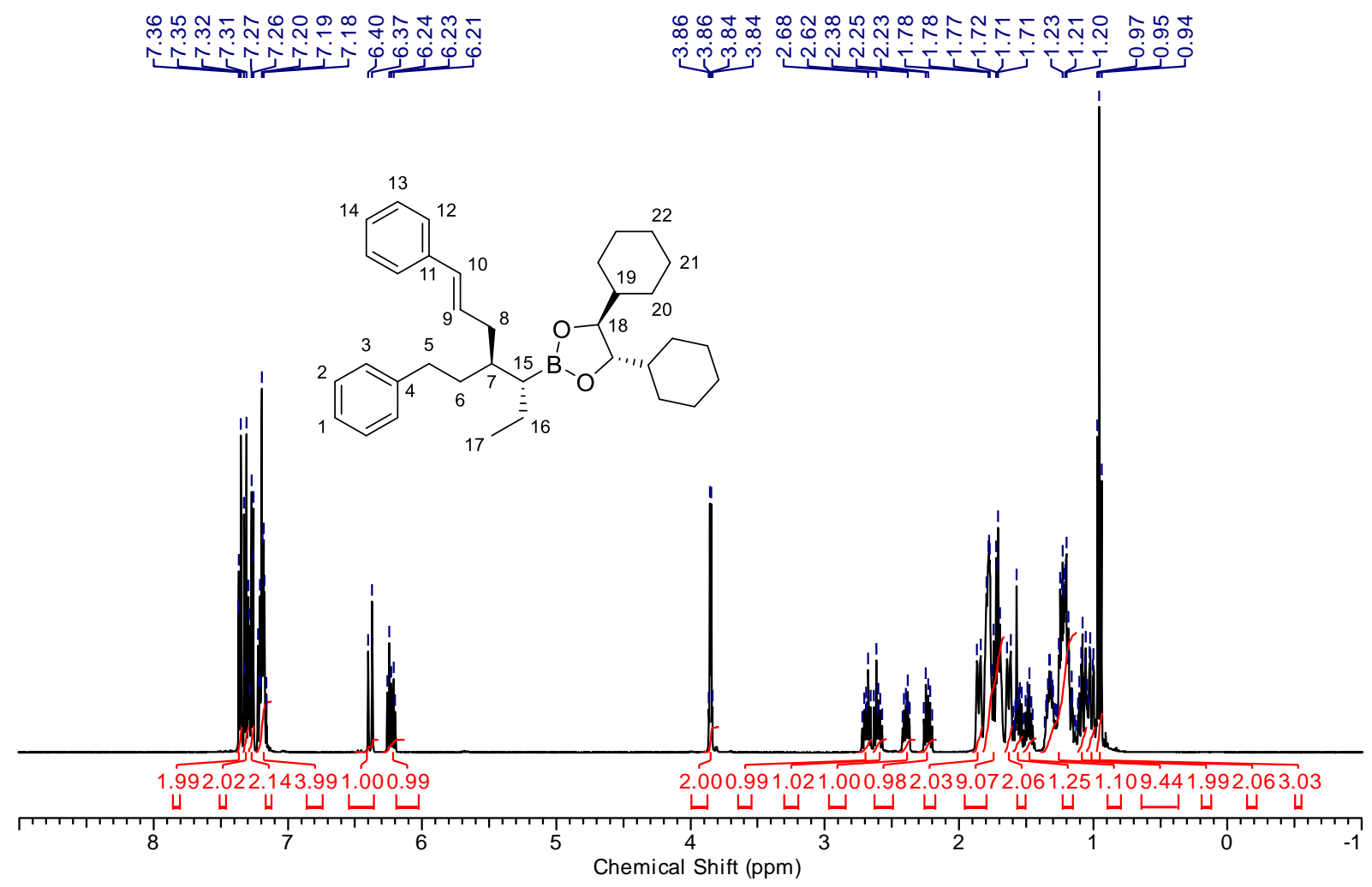

${ }^{13} \mathrm{C}$ NMR (125 MHz, $\left.\mathrm{CDCl}_{3}\right)$ :

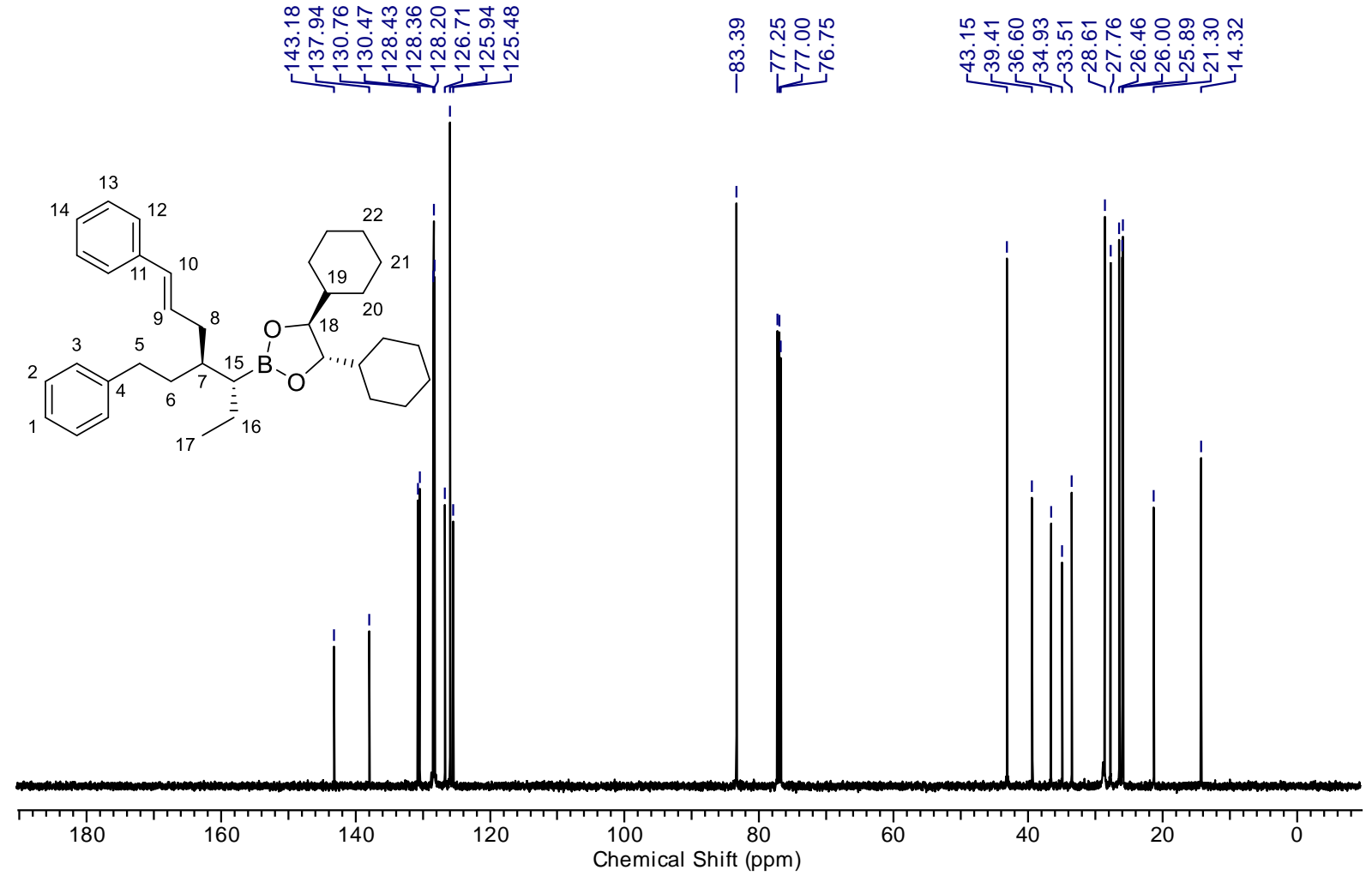


Potassium [(S,E)-1,6-diphenylhex-5-en-3-yl] trifluorborate (28)

${ }^{1} \mathbf{H}$ NMR (400 MHz, $\left.\mathrm{CD}_{3} \mathrm{CN}\right)$ :

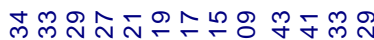

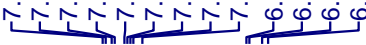

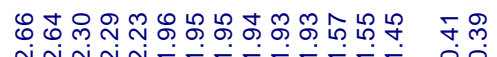

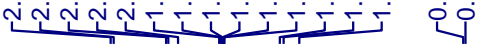

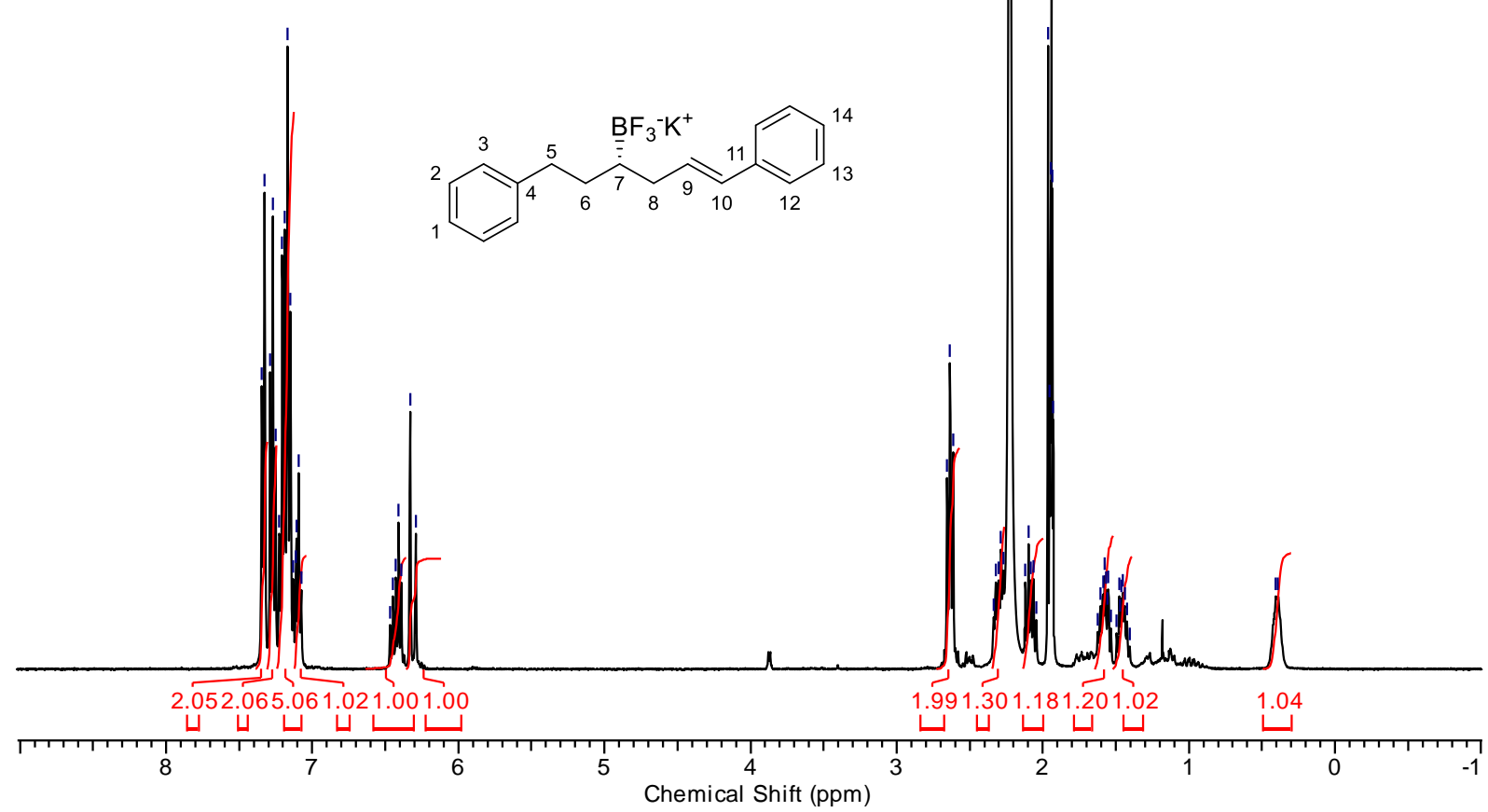

${ }^{13} \mathrm{C}$ NMR $\left(100 \mathrm{MHz}, \mathrm{CD}_{3} \mathrm{CN}\right)$ :

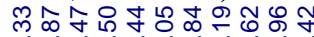

ஸ்

กฺุ

弚 மூர

둥유 윰

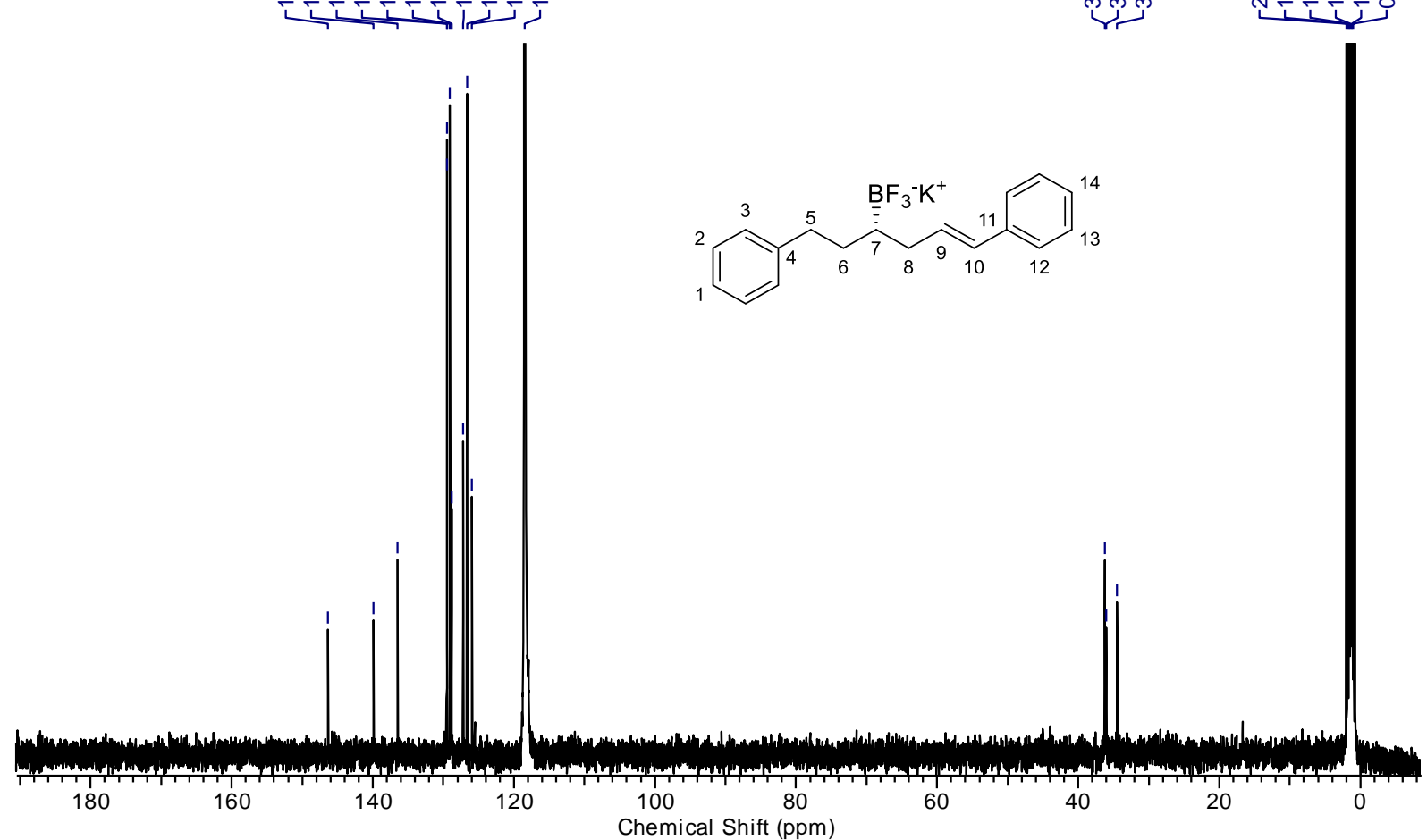


${ }^{11}$ B NMR (128 MHz, $\left.\mathrm{CD}_{3} \mathrm{CN}\right)$ :

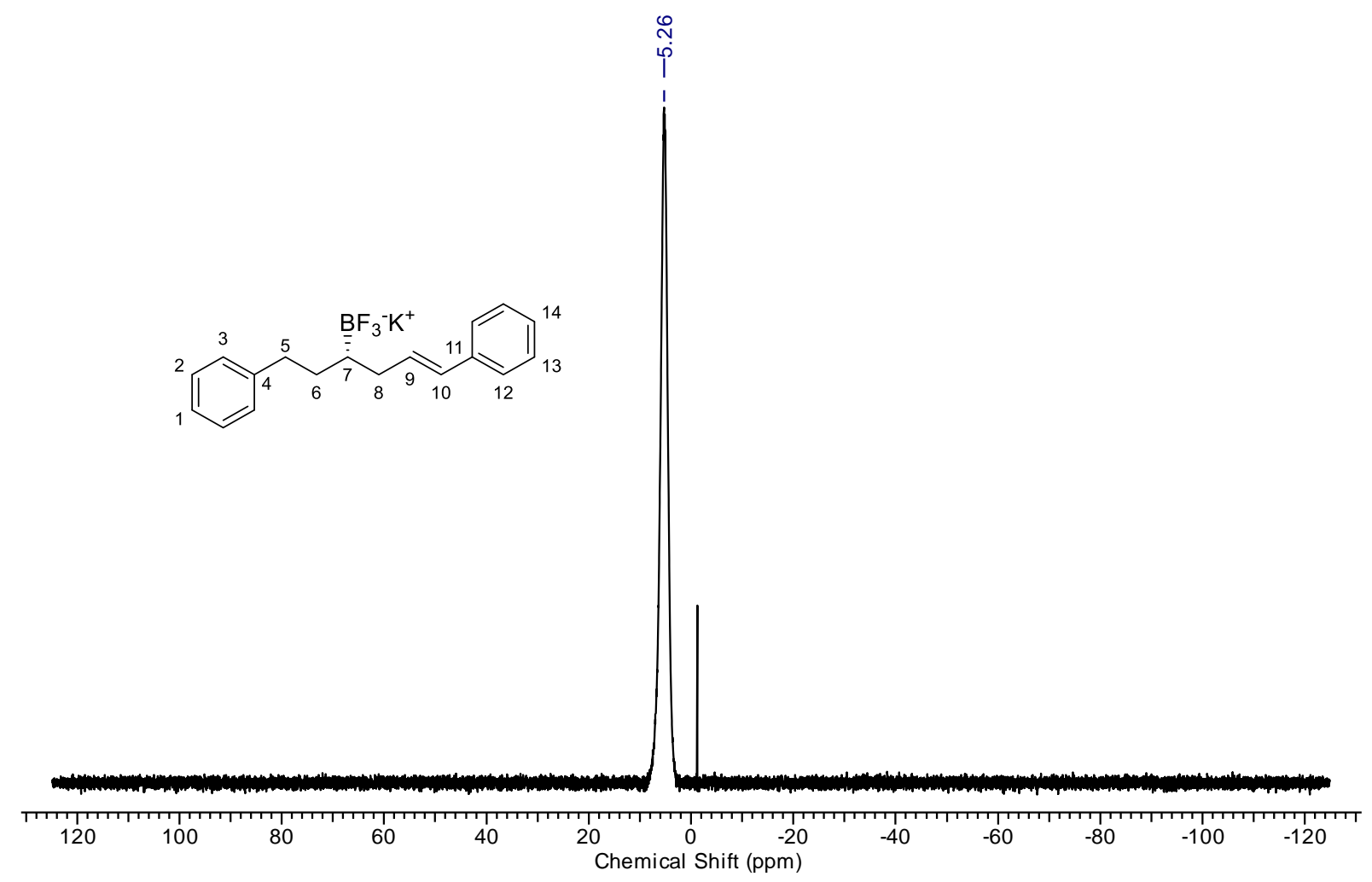


(S)-4-[(4S,5S)-4,5-Dicyclohexyl-1,3,2-dioxaborolan-2-yl]-6-phenylhexan-2-one (29a) ${ }^{1} \mathbf{H}$ NMR (400 MHz, $\left.\mathrm{CDCl}_{3}\right)$ :

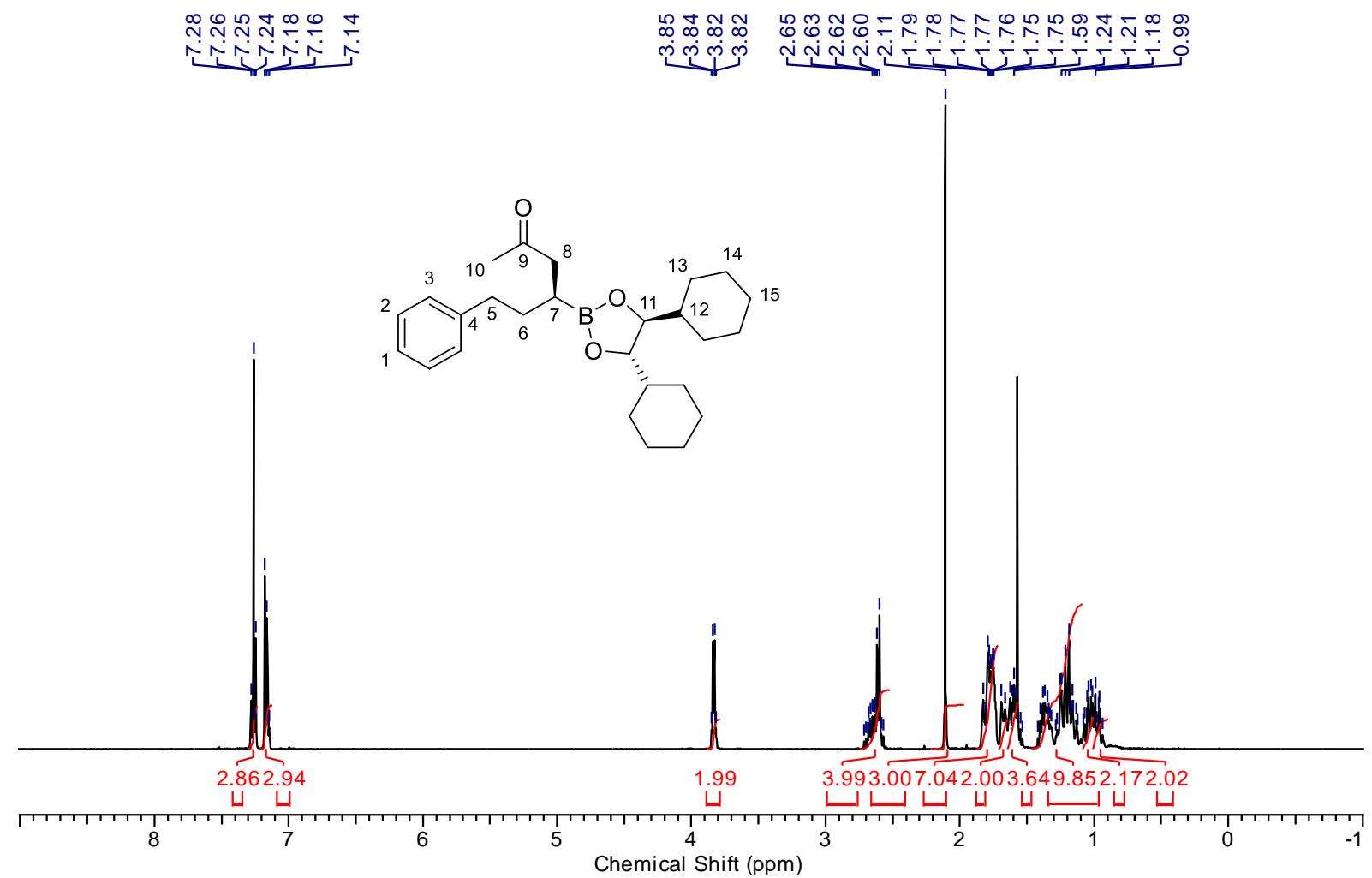

${ }^{13}$ C NMR (100 MHz, $\left.\mathrm{CDCl}_{3}\right)$ :
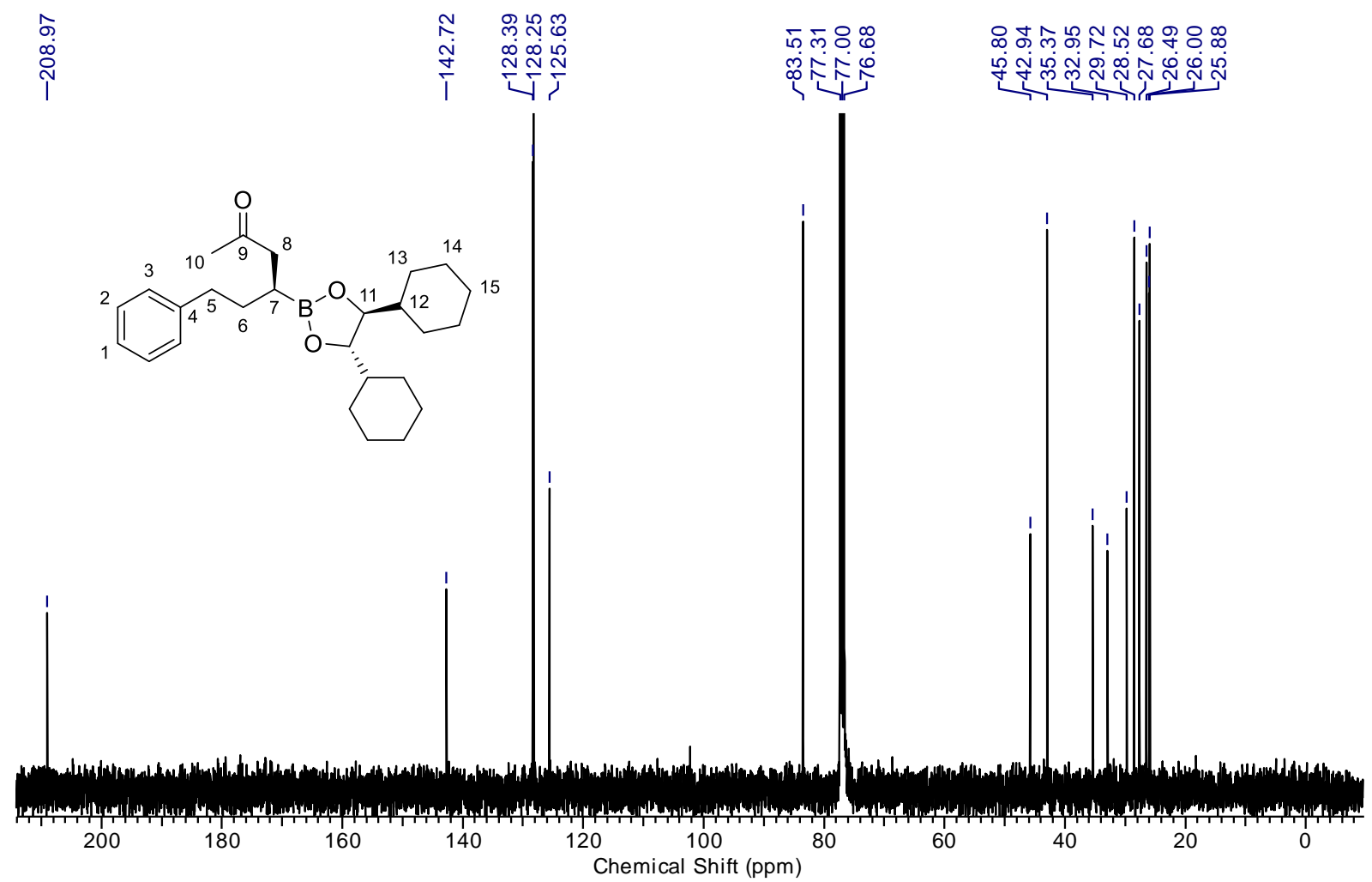
(S)-3-[(4S,5S)-4,5-Dicyclohexyl-1,3,2-dioxaborolan-2-yl]-5-phenylpentanal (29b) ${ }^{1} \mathbf{H}$ NMR (400 MHz, $\left.\mathrm{CDCl}_{3}\right)$ :

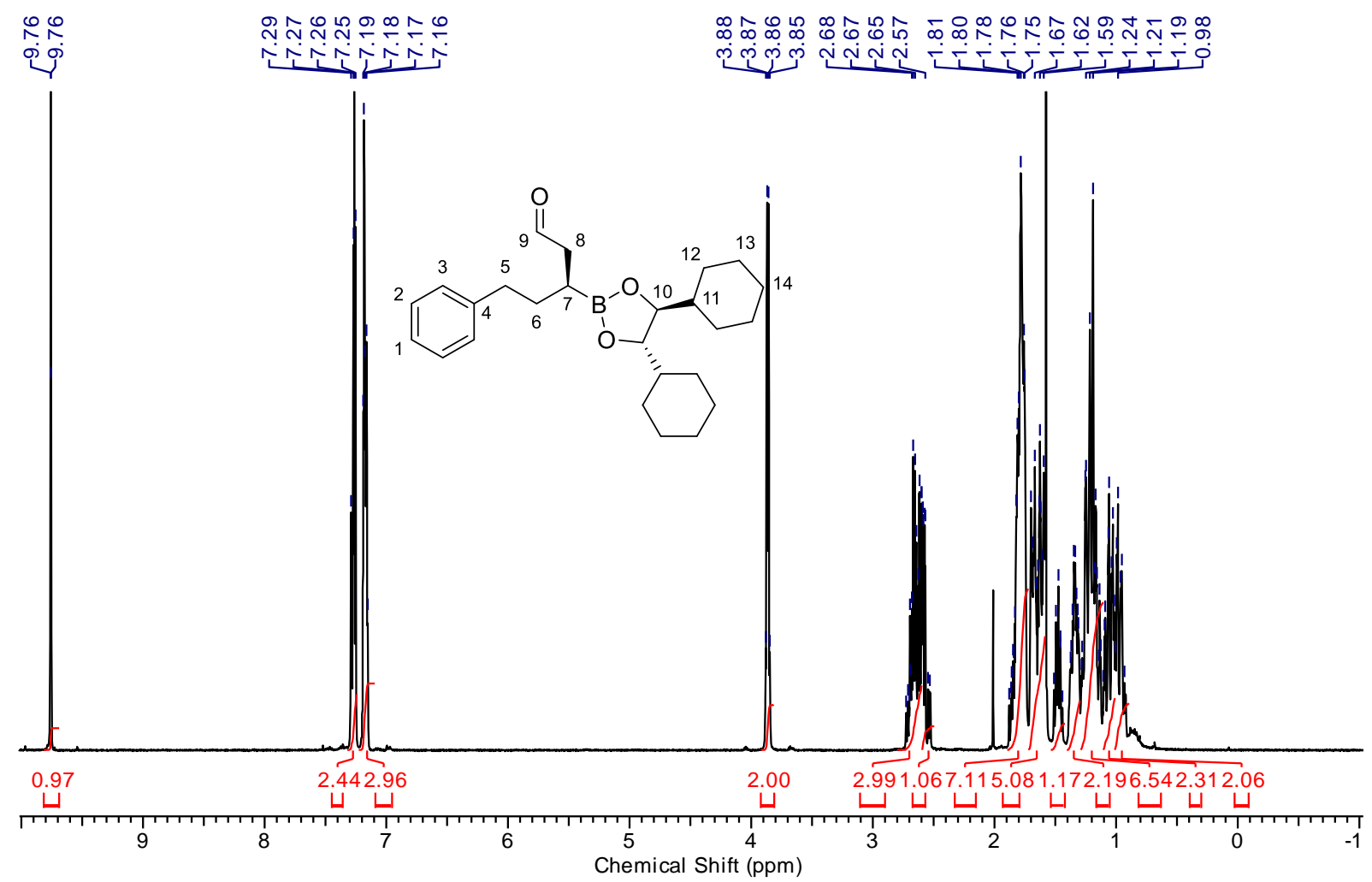

${ }^{13} \mathrm{C}$ NMR (100 MHz, $\left.\mathrm{CDCl}_{3}\right)$ :

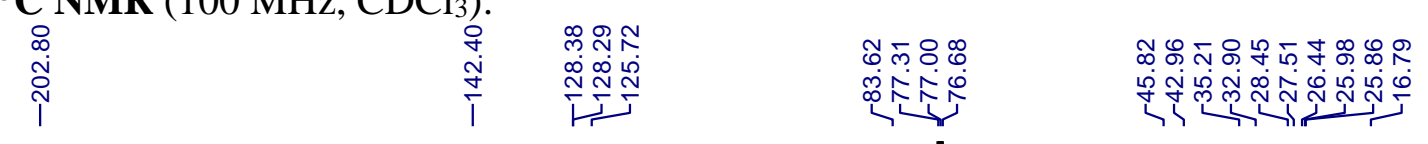

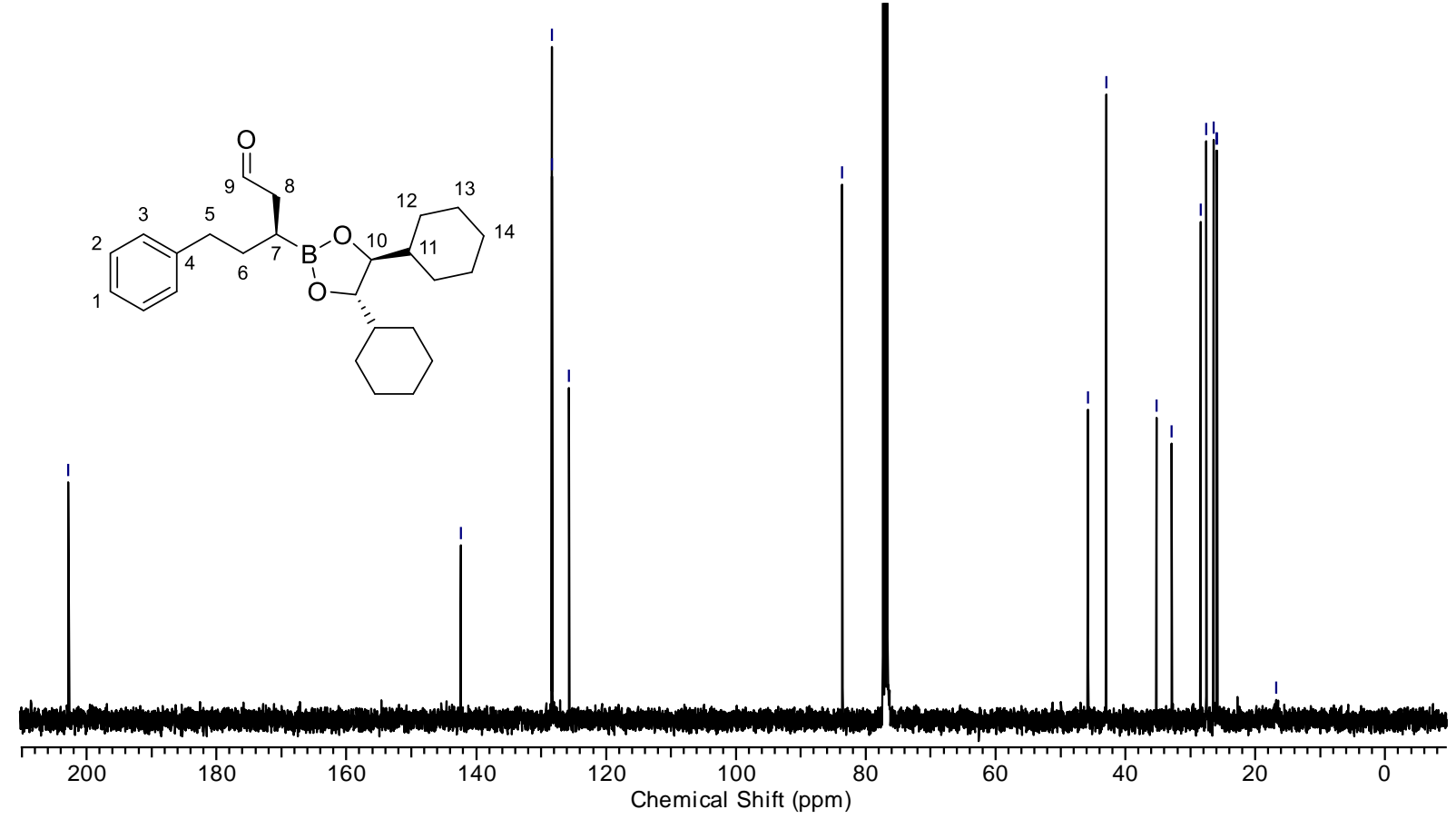




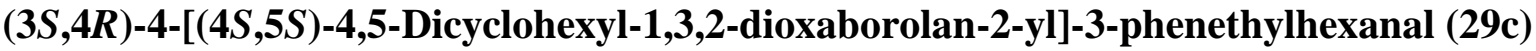
${ }^{1} \mathbf{H}$ NMR (400 MHz, $\left.\mathrm{CDCl}_{3}\right)$ :

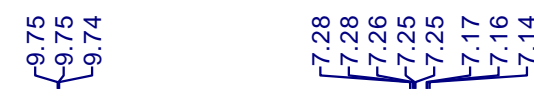

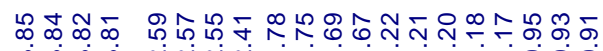

ijg

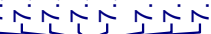

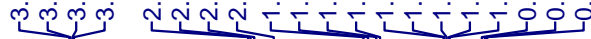<smiles>[Z]C(CCCc1ccccc1)B1OB(C2CCCCC2)OC(C2CCCCC2)O1</smiles>

${ }^{13} \mathrm{C}$ NMR $\left(100 \mathrm{MHz}, \mathrm{CDCl}_{3}\right)$ :

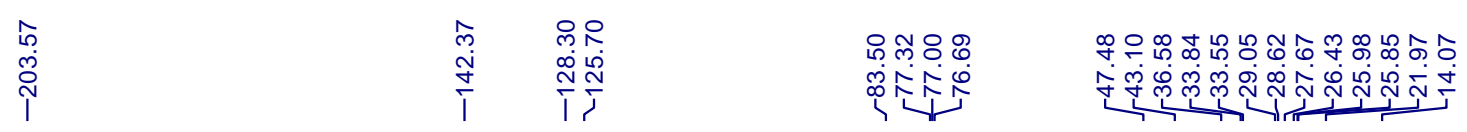

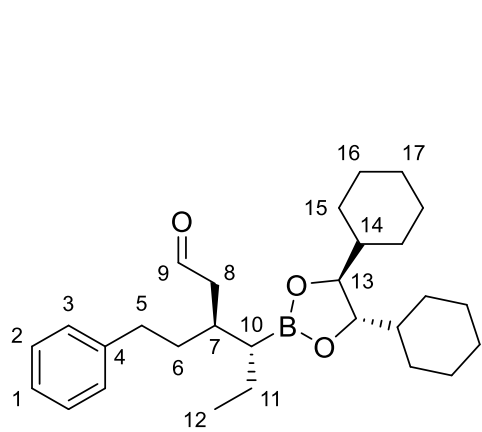

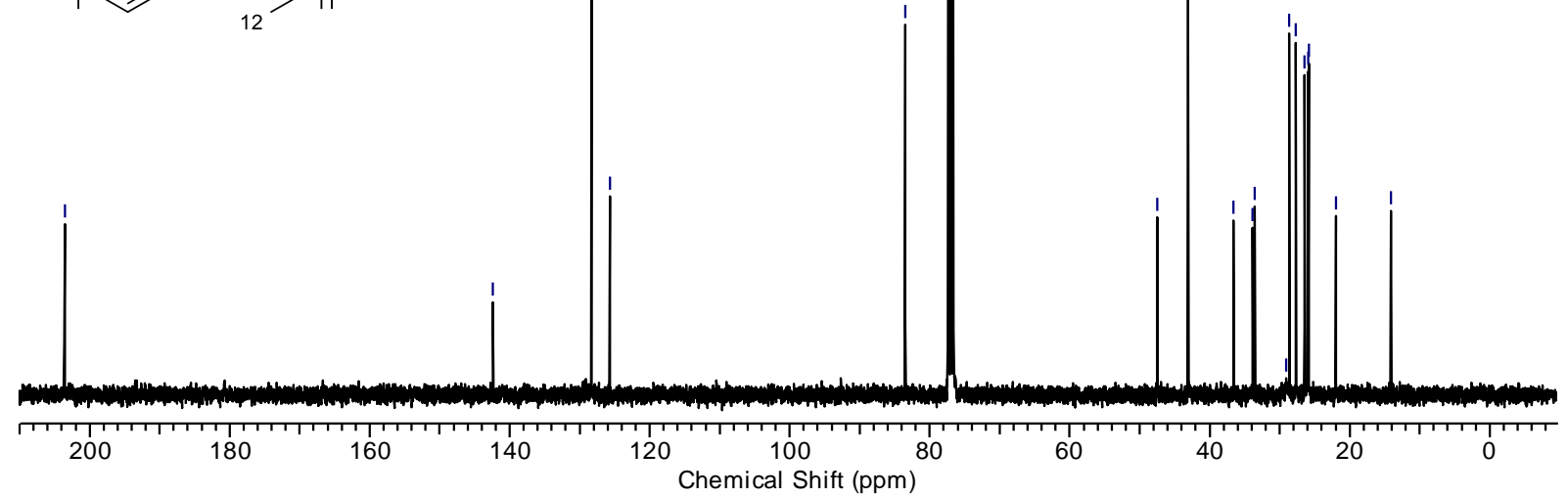


(S)-4-Hydroxy-6-phenylhexan-2-one (30a)

${ }^{1} \mathbf{H}$ NMR (400 MHz, $\left.\mathrm{CDCl}_{3}\right)$ :

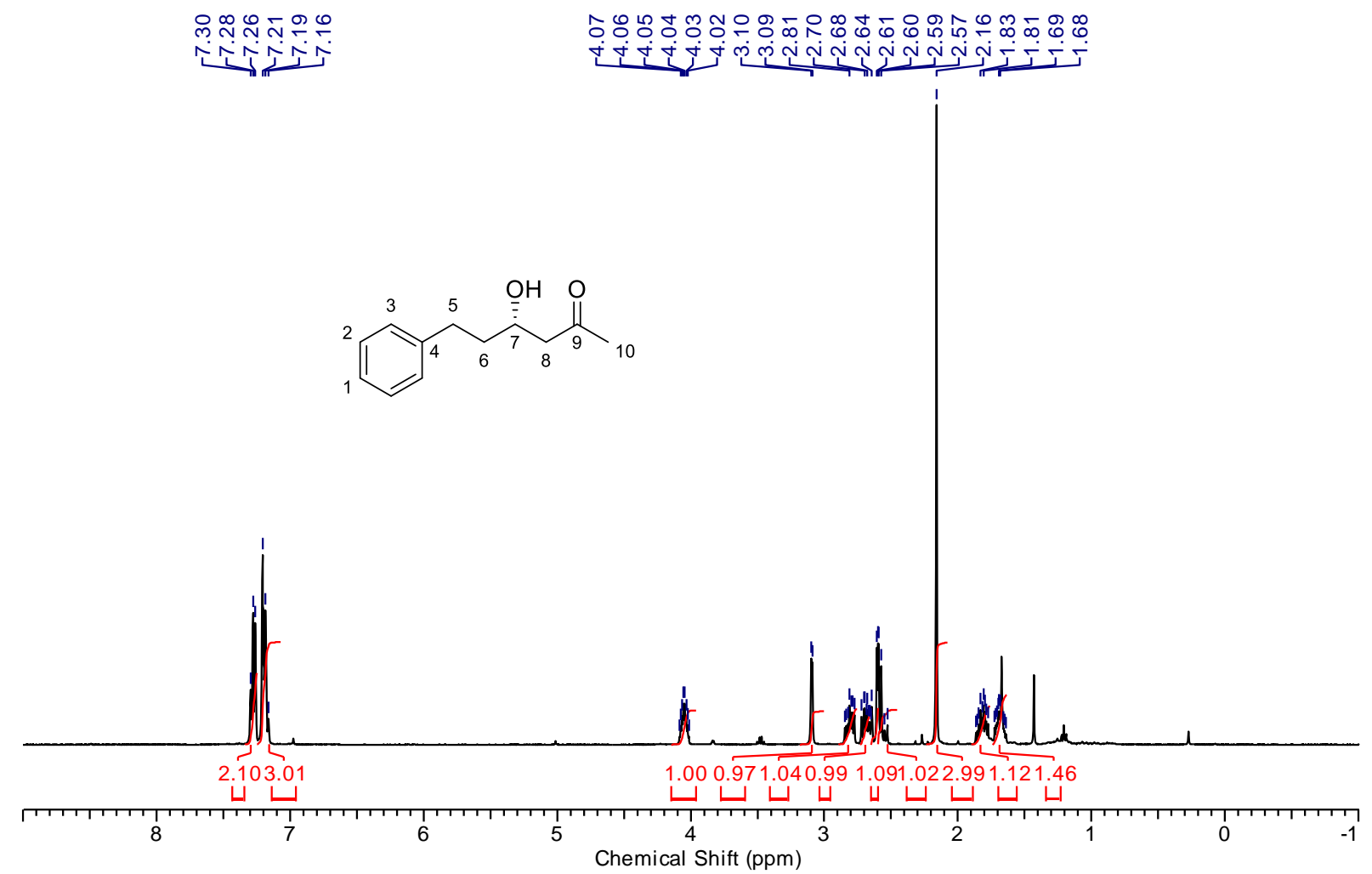

${ }^{13}$ C NMR (100 MHz, $\left.\mathrm{CDCl}_{3}\right)$ :

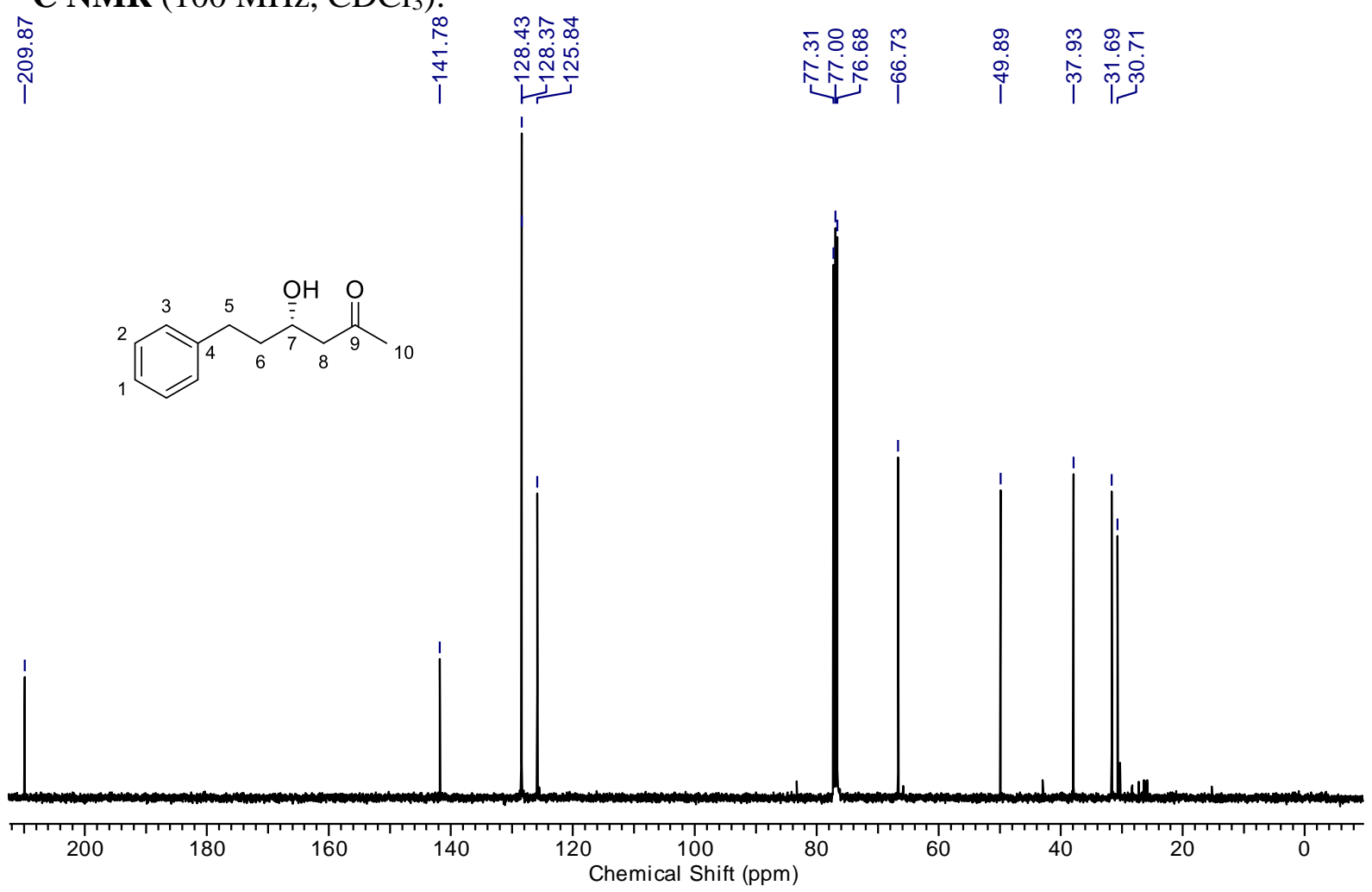


(4S,5R)-5-Ethyl-4-phenethyltetrahydrofuran-2-ol (30b)

${ }^{1} \mathbf{H}$ NMR $\left(500 \mathrm{MHz}, \mathrm{CDCl}_{3}\right)$ :

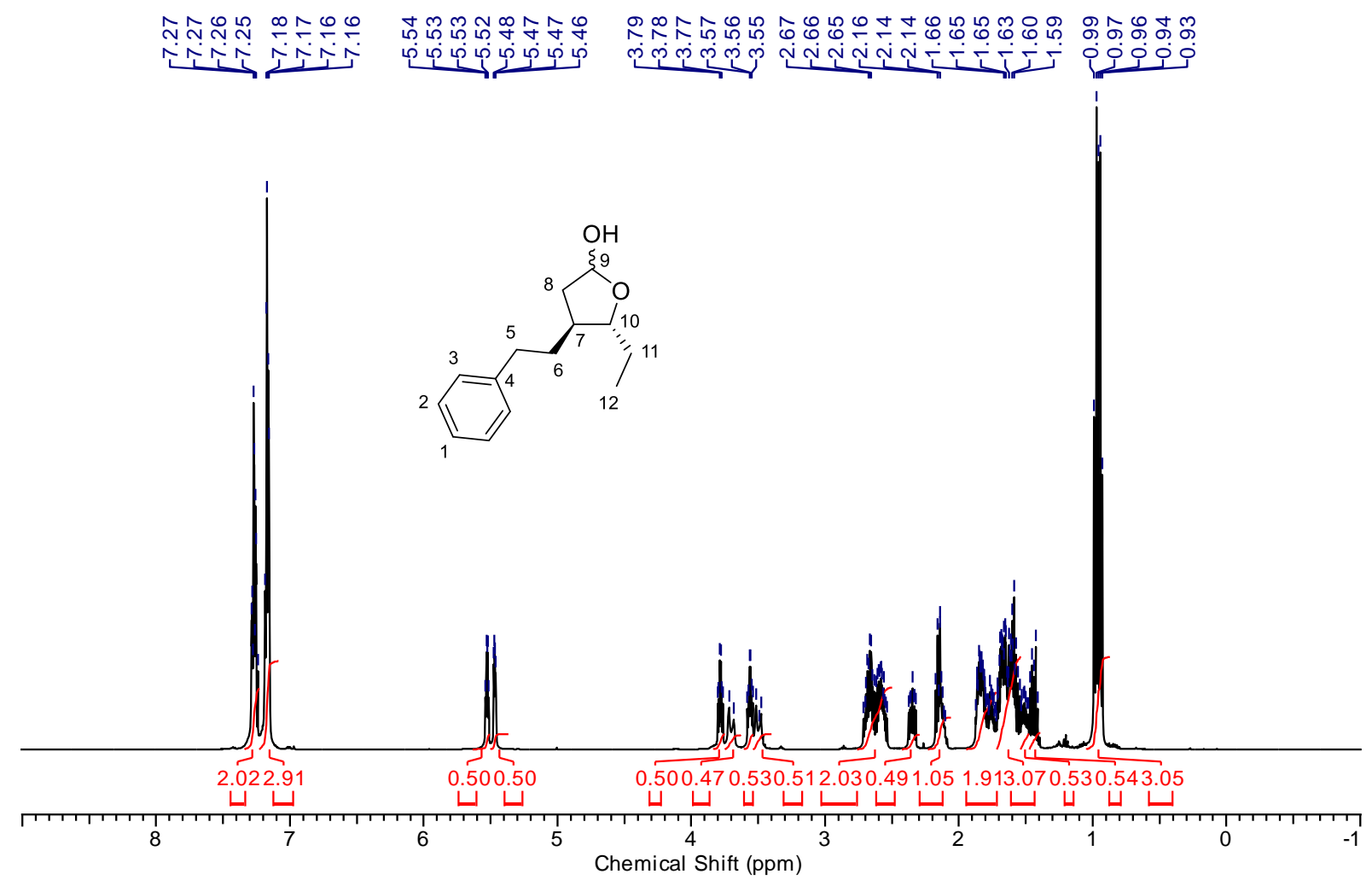

${ }^{13}$ C NMR (125 MHz, $\left.\mathrm{CDCl}_{3}\right)$ :

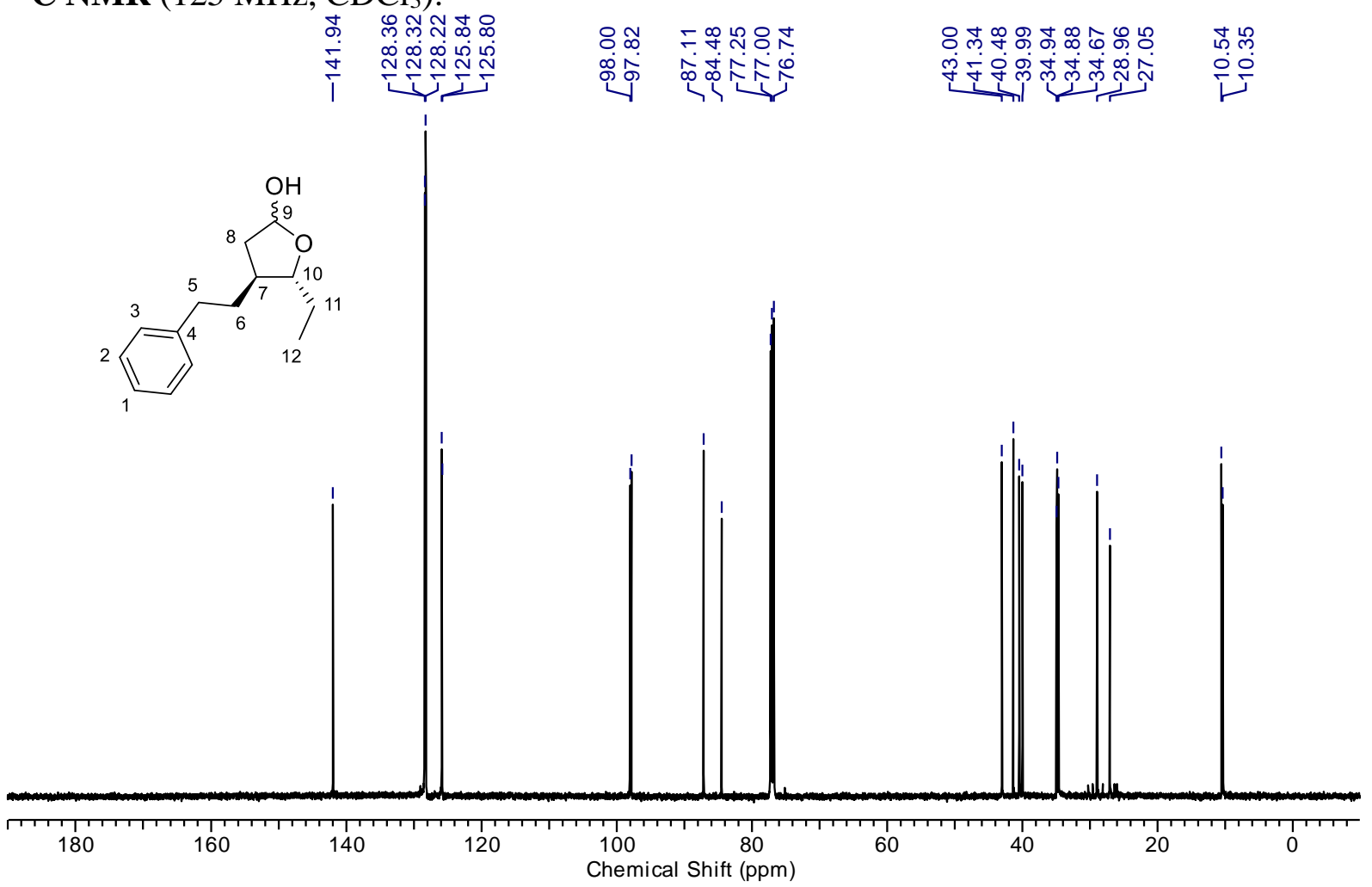


1D CSSF TOCSY (500 MHz, $\mathrm{CDCl}_{3}, 3.78$ ppm, signals of anomer 1):

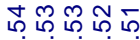

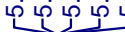
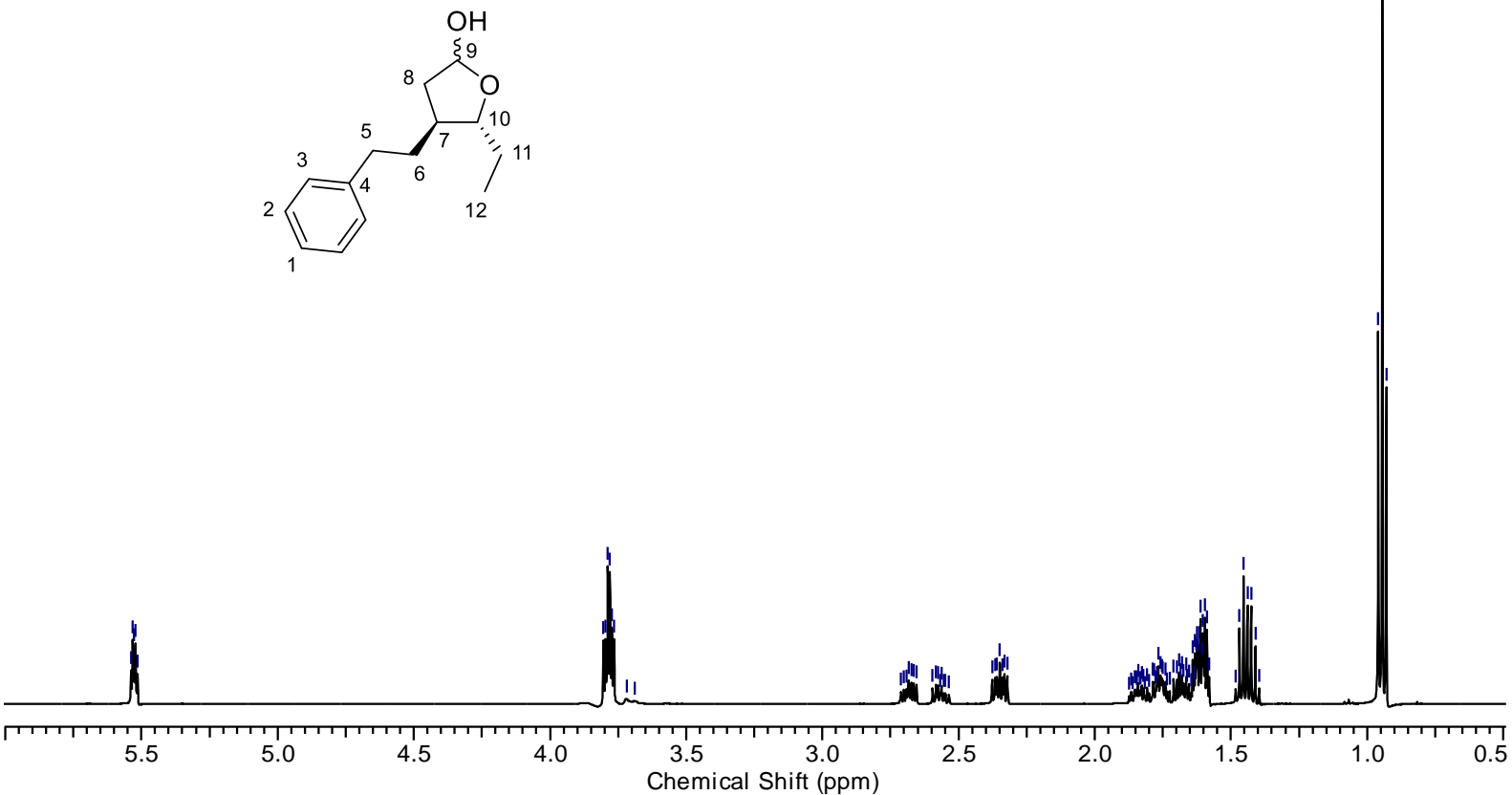

1D CSSF TOCSY (500 MHz, $\mathrm{CDCl}_{3}, 3.56 \mathrm{ppm}$, signals of anomer 2):

守寺寺

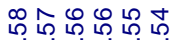

लंखुला

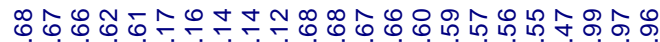

மน نุ

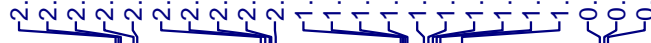
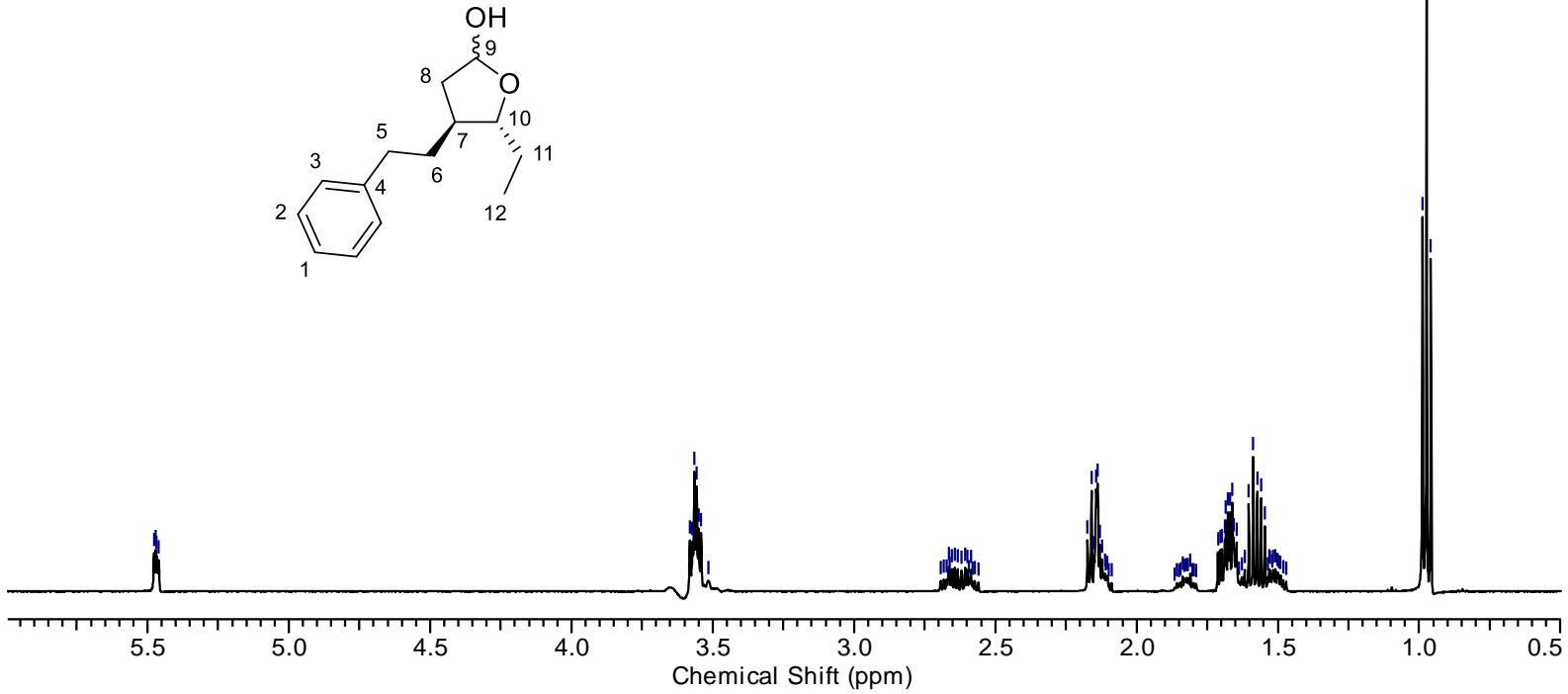